\title{
KINETIC BOUNDS ON ATTAINABILITY \\ IN \\ THE REACTOR SYNTHESIS PROBLEM
}

\author{
DISSERTATION \\ Presented in Partial Fulfillment of the Requirements for \\ the Degree Doctor of Philosophy in the Graduate \\ School of The Ohio State University
}

By

Thomas Kannankara Abraham, B.Tech.

$* * * *$

The Ohio State University

2005

Dissertation Committee:

Approved by

Professor Martin Feinberg, Adviser

Professor Bhavik Bakshi

Professor James Rathman

Adviser

Graduate Program in

Chemical Engineering 



\begin{abstract}
For a given chemical reaction network with known kinetics, a specified feed composition, and specific process constraints, the attainable region for the reactor synthesis problem is the set of all composition vectors that are achievable through all constraint-consistent reactor configurations that invoke only reaction and mixing. The boundary of the attainable region gives useful information to a process designer in terms of yield and selectivity of a desired chemical species that might be achieved by any constraint-consistent design. The process designer can use this information to assess the effectiveness of existing designs and consider alternate designs that might produce an effluent composition closer to the boundary of the attainable region.

Though much is known about necessary conditions for compositions to lie on the boundary of the attainable region, a definite method to calculate the true boundary of the attainable region is not known. Most of the existing methods compute "candidate" attainable regions by starting at the feed composition and attempt to enlarge the set of attainable compositions by considering various reactor configurations until no further enlargement is seen. These methods raise the question of whether the "candidate" attainable region has come close to the (currently unknown) true attainable region. These methods cannot preclude with certainty the existence of some novel reactor configuration that can enlarge the current set of achievable compositions.
\end{abstract}


This thesis describes a new technique called the method of bounding hyperplanes that complements existing methods by "bounding from the outside" those composition vectors that are attainable. In other words, the method seeks to create a set of composition vectors such that all composition vectors lying outside this set are inaccessible from the feed. Thus, the true attainable region will be contained in or will coincide within the bounding set so created.

The theoretical underpinnings for the method of bounding hyperplanes are described followed by algorithms to implement the method computationally. Kinetic bounds are computed by the method in several examples and compared with stoichiometric bounds and with compositions computed for various typical reactor designs. The kinetic bounds do well in precluding a large set of compositions that would otherwise be considered feasible from stoichiometric considerations alone. In many instances, the computed bounds are sharp insofar as they come very close to compositions known to be attainable. The methods described in this thesis do not replace existing methods to find candidate attainable regions but rather complement them. 


\section{Dedicated,}

To my late grandparents (Appachan \& Ammachy).... for all their love and constant prayer To my parents (K T Abraham \& Annamma).... for their hopes and the confidence they have in me, that has made me who I am

To my sister (Annie)....for being more than a sister to me

To my wife (Anita) and my daughter (Rebecca)....for being my constant source of love, support, inspiration and motivation while writing this thesis

$\&$

For the Lord Jesus' glory alone 


\section{ACKNOWLEDGMENTS}

I would like to place on record my heartfelt gratitude to my adviser Dr Martin Feinberg for his extraordinary intellectual support, encouragement, teaching, patience and faith in me, which made this thesis possible. His training has improved my ability to think independently and I am indebted to him for his efforts that made my work as close to perfect as possible.

I would like to thank the National Science Foundation (NSF) that funded the research presented in this thesis and acknowledge the OSU Research Foundation for permitting access to the NSF grant that made various equipment purchases and travel funding possible. I am thankful to the Department of Chemical \& Biomolecular Engineering at OSU for providing the opportunity and infrastructure to study and conduct research.

I am greatly indebted to Phillipp Ellison for his support during the initial period of my research. My sincere thanks to my group-mate Yangzhong Tang for her help with the case studies and in many other ways during my research.

I am extremely thankful for the assistance received from Brad Barber (formerly in the Geometry Center of U. of Minnesota) whose qhull program I modified to obtain the beautiful 3-D plots for the case studies. Part of the credit for this also goes to Ms. Natalia 
Platinova. I appreciate the assistance provided by Komei Fukuda (ETHZ) as I toiled with cdd and cddlib. Besides google.com and Google groups where I found many solutions to programming problems, I am also indebted to Eric (deltatrinity) who helped me compile GMP in Borland C++ Builder. I appreciate the suggestions from Michal Kvasnica and Torrisi Fabio of ETHZ that helped modify cddlib for our requirements.

Lastly, but not the least, I am grateful to each and every member of my family who has shown their love and support in some form or the other. I am forever thankful for my late grandparents who took care of me as an infant and later when I was an engineering student. I am thankful for my amazing parents and my sister for their constant love and support throughout my life. I am indebted to my wife of more than a year and our 3-month- old daughter without whom I might not have completed this thesis on time. Finally, I sincerely acknowledge that my intellect and all my achievements have been through God's abundant grace and mercy alone. 
VITA

October 25, 1976.....................Born - Abu Dhabi, U.A.E.

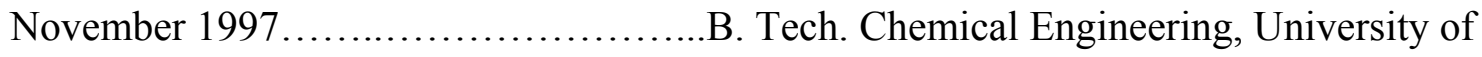
Kerala (T K M College of Engineering), Kerala, India

February 1998- December 1998...........Process Engineer, Asian Paints (India) Ltd Cuddalore, India

March 1999- August 1999................Chemical Engineer, Al Husam General Contracting, Abu Dhabi, U.A. E.

October 1999-December 2000............Graduate Teaching Associate, Dept. of Chemistry, The Ohio State University

January 2001-present...................Graduate Research Associate, Dept of Chemical and Biomolecular Engineering, The Ohio State University

\section{PUBLICATIONS}

Research Publication

Abraham, T. K.; Feinberg, M., Kinetic Bounds on Attainability in the Reactor Synthesis Problem. Industrial and Engineering Chemistry Research 2004, 43, (2), 449-457.

\section{FIELDS OF STUDY}

Major Field: Chemical Engineering 


\section{TABLE OF CONTENTS}

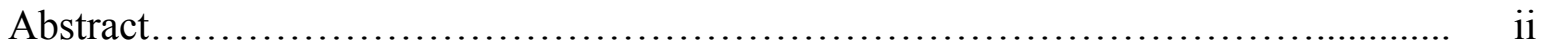

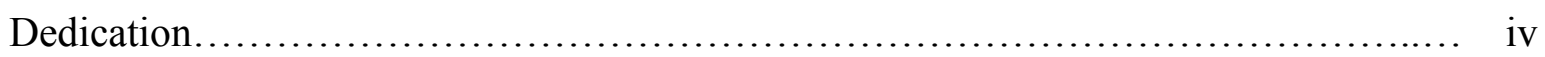

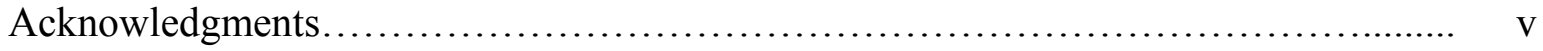

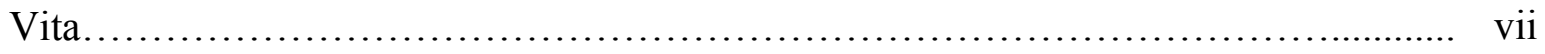

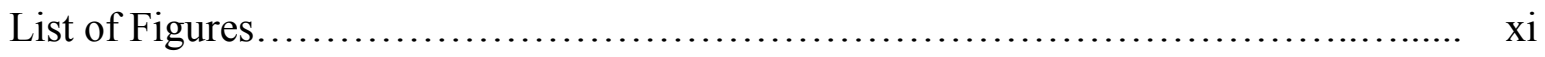

List of Abbreviations............................................................. xvi

Chapters:

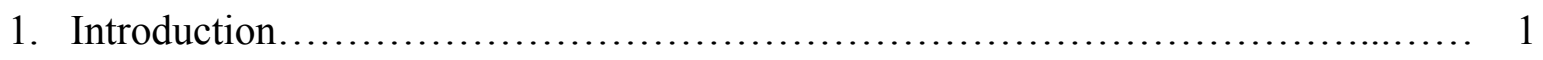

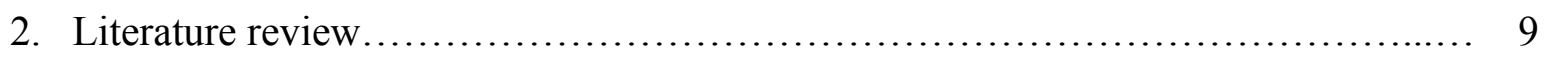

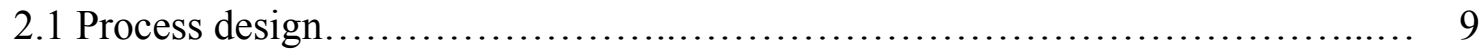

2.2 Process synthesis in general............................................... 10

2.3 Computational Techniques................................................. 11

2.4 Process synthesis software ................................................ 15

2.5 Reactor synthesis with and without separators.............................. 16

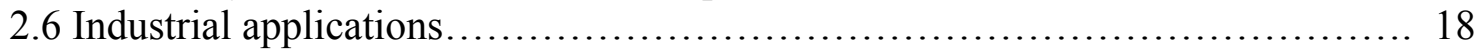

3. The attainable region approach to the pure-reactor synthesis problem and its short

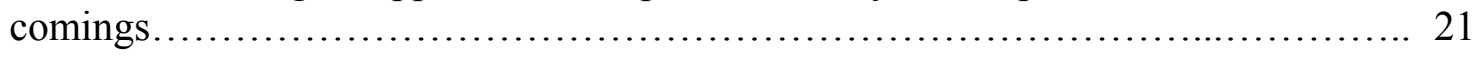

3.1 Comparing the pure-reactor and the reactor-separator synthesis problems......... 21

3.2 The pure-reactor synthesis problem........................................ 23

3.3 Universal properties of the attainable region................................. 26

3.4 Critical CFSTRs............................................................. 31

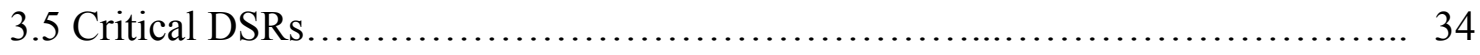


3.6 Analyzing the "critical" design equations................................... 36

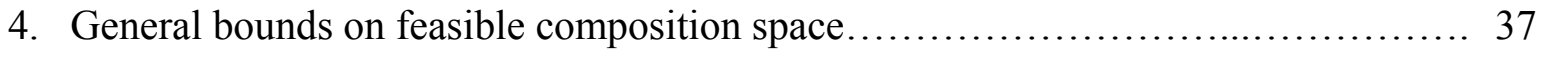

4.1 Mathematical preliminaries................................................. 37

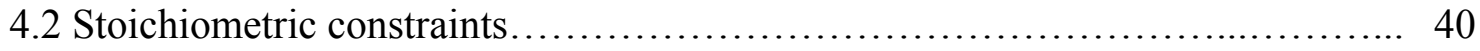

4.2.1 Compositions stoichiometrically compatible with the feed................. 43

4.2.2 Methods to compute stoichiometric constraints...................... 47

4.3 Thermodynamic bounds...................................................... 50

4.4 Reactor-separator bounds................................................... 55

5. The method of bounding hyperplanes........................................ 61

5.1 Preliminary ideas, terminology and notation..................................... 61

5.2 Introduction to the method of bounding hyperplanes.......................... 65

5.3 Mathematical justification for the technique................................. 71

5.4 Treatment of multiple feed stream systems ............................... 77

6. Implementation techniques....................................................... 78

6.1 Preliminary ideas about polyhedral computation.............................. 78

6.2 Maple implementation and algorithms..................................... 86

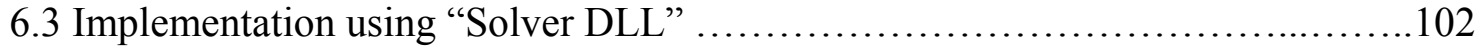

6.4 Limitations of the Solver DLL implementation.............................. 117

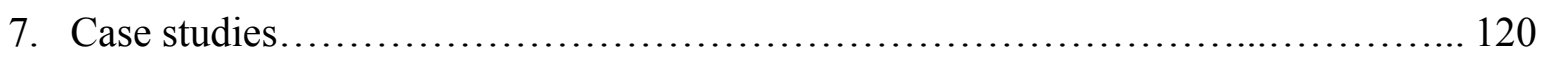

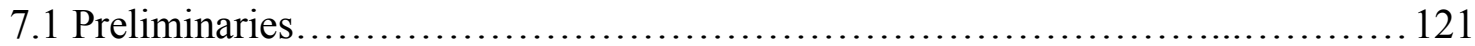

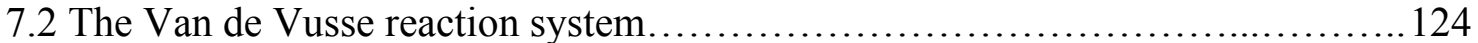

7.2.1 The irreversible Van de Vusse Example................................ 125

7.2.2 Reversible Van de Vusse example........................................ 139

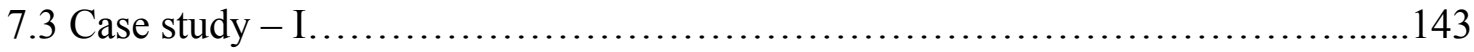

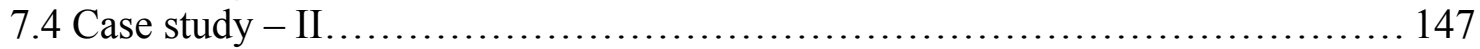

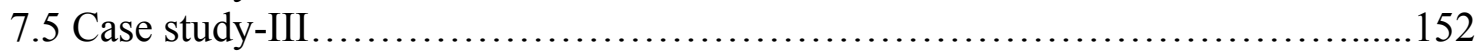

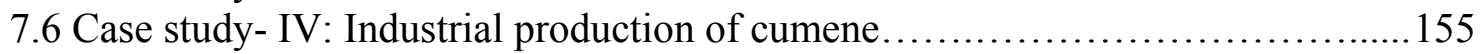

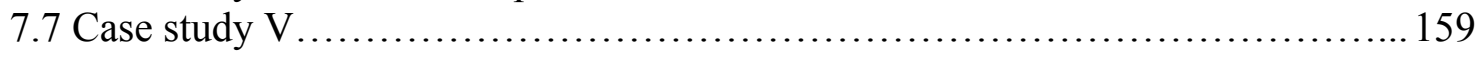

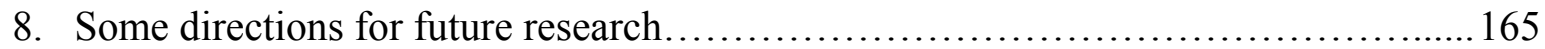


8.1 Implementation challenges............................................... 166

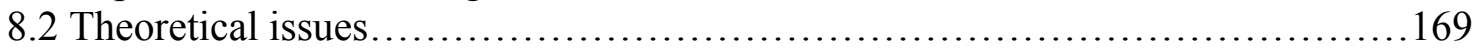

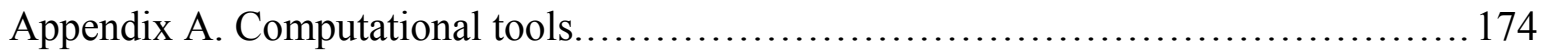

A.1 cddlib.................................................................... 174

A.2 GNU multiple precision arithmetic library (GMP) .......................176

A.3 Solver dynamic link libraries (Solver DLL) ........................... 177

A.3.1 Small-scale solver dll..................................... 178

A.3.2 LGO global solver engine.................................... 180

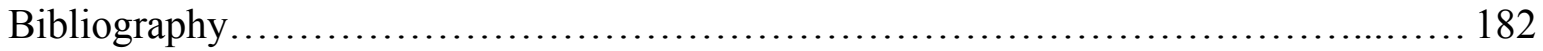




\section{LIST OF FIGURES}

Figure $\quad$ Page

1.1 Some achievable compositions (in moles per unit volume) for the 3-D van de Vusse problem....................................................4

3.1 Possible reactor configurations for the pure reactor synthesis problem.........25

3.2 Manifold of extreme points meeting a straight line section smoothly.............27

3.3 Manifold of extreme points meeting a straight line section roughly.............28

3.4 PFR composition trajectories that reside on the manifold of extreme points.....29

3.5 Reactor configurations that shape the boundary of the attainable region where the smooth-line section meets the protrusion smoothly ..........................31

3.6 Schematic of a DSR ..................................................

4.1 A tetrahedron in $\mathbb{R}^{3}$ of compositions stoichiometrically compatible with the feed....

4.2 Comparing thermodynamic constraints and stoichiometrically feasible compositions for a feed stream composition $[1,0]$

4.3 Comparing thermodynamic constraints and stoichiometrically feasible compositions for a feed stream composition of $[0.5,0.5]$.

4.4 Comparison of a set of achievable compositions with compositions corresponding to the full set of attainable reactor-separator molar effluent rates

5.1 A single bound-trimming sequence on a composition set...................66

5.2 An impossible situation : No process design utilizing reaction and mixing alone with a feed stream can reach the composition in the manner shown.... 
5.3 A second trimming sequence on a previously reduced composition set..........68

5.4 Complete bound trimming sequence for $p$ stages...........................69

5.5 A hyperplane dividing the current composition set into two parts: one part containing the feed and in the other part all the rate vectors(shown as small directional arrows) at nonequilibrium compositions point toward the side containing the feed...................................................... 72

6.1 Example to describe the two representations that describe a convex set..........80

6.2 An example of redundant inequalities in a system of inequalities...............82

6.3 Hyperplanes with different angle parameters $\ldots \ldots \ldots \ldots \ldots \ldots \ldots \ldots \ldots \ldots \ldots . \ldots . \ldots \ldots$

6.4 Algorithm for the Maple implementation of the method of bounding hyperplanes....................................................... 89

6.5 A fragment of the current polytope showing computation at an extreme point....91

6.6 A fragment of the current polytope showing a new hyperplane being "moved inside" it.............................................................92

6.7 A grid to determine points residing on a hyperplane........................94

6.8 An example showing the creation of grid points and moving of a hyperplane....96

6.9 Example showing how discretization could "miss" compositions of interest.....100

6.10 Example showing how the hyperplane step size might cause inadvertent skipping of interesting compositions........................................... 101

6.11 Program structure for the Solver DLL implementation of the method of bounding hyperplanes.................................................... 104

6.12 Figure showing lower limit and upper limit added to the current polytope as part of initialization in the Solver DLL module... 
6.13 Iterations in the Solver module showing constraints being moved such that the optimization indicates those regions that are infeasible.

6.14 A simple function plot showing several local optima (and a single global optimum)

7.1 Examples for a convex set, non-convex set and convex hull.................122

7.2 Plot showing compositions that are stoichiometrically compatible with the feed for the $2 \mathrm{D} \mathrm{VdV}$ problem..............................................126

7.3 Plots showing implementation in Maple of the method of bounding hyperplanes. This is the $2 \mathrm{~d}-\mathrm{VdV}$ example starting with stoichiometric constraints and adding more hyperplanes that trim bounds on achievable composition space..........128

7.4 Iterations with a large number of hyperplanes showing how more curvature is approximated through more hyperplanes...............................129

7.5 Superimposing of two plots (1) Black lines indicating hyperplanes computed by the Maple implementation (2) Curves representing CFSTR loci and PFR trajectories that is believed to be the full set of achievable compositions in the $2 \mathrm{~d}$ VdV problem.................................................... 130

7.6 Solver Implementation output for the $2 \mathrm{~d}$ VdV problem (cream-brown lines) superimposed on compositions computed for reactor configurations believed to be the full set of achievable compositions. 131

7.7 Tetrahedron of compositions that are stoichiometrically compatible with the feed for the $3 \mathrm{D} \mathrm{VdV}$ problem............................................ 132

7.8 Comparison of bounds computed by Maple with compositions from reactor designs suggested by general properties of the attainable region (magnified view).

7.9 Unmagnified view of a set of bounds computed by Maple sandwiched between stoichiometric constraints (dotted line) and a set of achievable compositions computed for the $3 \mathrm{~d} \mathrm{VdV}$ problem. 
7.10 Constraints from 422 hyperplanes compared with a set of achievable compositions for the $3 \mathrm{dVdV}$ problem as suggested in the literature.

7.11 Unmagnified view of a set of bounds computed by Solver DLL implementation sandwiched between stoichiometric constraints (dotted line) and a set of achievable compositions computed for the $3 \mathrm{~d} V \mathrm{dV}$ problem 138

7.12 Comparing stoichiometric constraints, kinetic bounds and a set of achievable compositions for the reversible Van de Vusse reaction network

7.13 Magnified view of the kinetic bounds compared with some achievable compositions computed for various CFSTR residence times (in red) and PFR configurations (in green).

7.14 Case Study I- Comparing the stoichiometric constraints and kinetic bounds (computed using the Solver DLL implementation)....

7.15 Case Study I - Comparing the kinetic bounds with CFSTR loci and various PFR trajectories

7.16 Comparison of stoichiometric constraints, kinetic bounds and a set of achievable compositions.... 149

7.17 A set of achievable compositions with a combination of DSR and PFR trajectories

7.18 Figure showing how more hyperplanes are required to approximate curvature..151

7.19 A set of achievable compositions in comparison with kinetic bounds computed by Solver DLL implementation................................................154

7.20 Comparison of the stoichiometric constraints, kinetic bounds and a set of achievable compositions.

7.21 First plot showing feed composition and compositions that are stoichiometrically compatible with the feed. Second plot shows kinetic bounds computed by Solver DLL implementation. 
7.22 Comparing kinetic bounds with a set of feasible compositions computed for various CFSTR and PFR designs...............................................159

7.23 Comparing stoichiometric constraints, kinetic bounds and a set of achievable

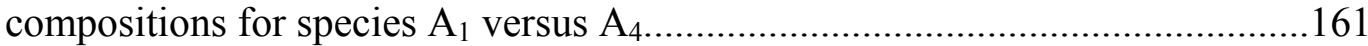

7.24 Comparing stoichiometric constraints, kinetic bounds and a set of achievable

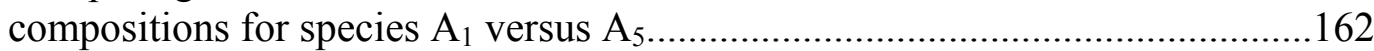

7.25 Comparing stoichiometric constraints, kinetic bounds and a set of achievable compositions for species $A_{1}$ versus $A_{6}$. A magnified view of the achievable

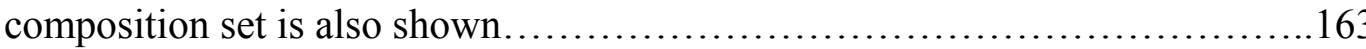




\title{
LIST OF ABBREVIATIONS
}

\author{
$\mathrm{AR}$ - Attainable region \\ CFSTR - Continuous-flow stirred tank reactor \\ DLL - Dynamic link library \\ DSR - Differential side-stream reactor \\ GMP - GNU multiple precision arithmetic library \\ GRG - Generalized reduced gradient \\ LGO - Lipschitz-continuous global optimization \\ MILP - Mixed-integer linear programming \\ MINLP - Mixed-integer nonlinear programming \\ NLP - Nonlinear programming \\ PFR - Plug-flow reactor \\ VdV - Van de Vusse (reaction network)
}




\section{CHAPTER 1}

\section{INTRODUCTION}

Chemical process design has become less of an art and more of a structured science over the past few decades. After the designer has set broad goals on what to produce, one of the preliminary steps of process design is to evaluate feasible designs from among a large number $\left(10^{4}\right.$ to $10^{9}$ according to Douglas[1]). Bad choices of process design during the early conceptual stages can lead to significant loss of time and revenue further along the design task. Process synthesis deals with methods to determine the overall structure of a process and the individual components that constitute the process.

A good design for a process is eventually one that gives the optimum profit for the desired operating conditions and various operating and/or environmental constraints imposed on it. The open ended nature of process design problems can lead to more than one solution and further analysis is needed to narrow down the choices. Improvements to an existing process design are usually limited to the creativity of the designer in coming up with a better design. But the ultimate analysis of any process design involves ascertaining if any changes to the process design will increase the profitability of the process. 
The attainable region concept was first introduced by F J M Horn [2] while at the Imperial College in London. This concept can be stated thus: For a given chemistry, a prescribed set of feed streams, and a prescribed set of process constraints, the attainable region is the complete set of output states that may be realized by all possible designs consistent with the system constraints. For a process designer, the attainable region provides information on the full range of process outcomes that the designer might ever achieve.

For a given system, knowledge of the attainable region assists one in assessing the relative merits of various design candidates. If a certain process design is found to be profitable and if the yield of that design is close to the most desirable yield that can be achieved through any constraint-consistent design, then one may not recommend investing time and effort in changing the design substantially. Alternatively, if a design is not found to be very profitable and it is known that its yield falls substantially short of the best possible yield, then further changes in the design may be justified. In either case, knowledge of the attainable region gives the designer an idea about what is achievable and what is not.

The goal of this thesis is to analyze the pure reactor synthesis problem. The focus is on the ability to describe the set of achievable output states for systems that utilize reactions and mixing alone without any separators. The designs are limited to steady-state systems that involve mixtures having a fixed density independent of composition. The methods developed in this thesis are restricted to isothermal systems though the ideas can be extended to treat non-isothermal systems as well. Isothermal systems have fixed reaction rate constants and are therefore amenable to easier analysis 
than non-isothermal systems where the rate constants might vary with changes in temperature.

Various researchers have tackled the reactor synthesis problem by attempting to build the attainable region "from the inside." Beginning with a feed stream composition, one would try to extend the set of achievable composition states by repeatedly invoking reactors having a feed stream from a set of already achieved composition states. The technique thereafter is to continue to add reactors until no additional compositions can be achieved. Further review of this literature is dealt in Chapter 2.

A frequently studied system, which was first introduced by Van de Vusse [3], can be described by the mass-action system show in eqn. (1.1)

$$
\begin{aligned}
& \underset{\mathrm{A}_{1}}{\stackrel{1}{\rightarrow} \mathrm{A}_{2}} \stackrel{1}{\rightarrow} \mathrm{A}_{4} \\
& 2 \mathrm{~A}_{1} \stackrel{10}{\rightarrow} \mathrm{A}_{3}
\end{aligned}
$$

The feed stream to the system can be described by the vector of molar concentrations $\mathbf{c}^{\mathbf{f}}=[1,0,0,0]$ in units consistent with the rate constants used. The interest is in the possible effluent concentrations of $A_{1}, A_{2}$ and $A_{3}$ for a system permitting reactions and mixing alone. In other words, we would like to know the full set of attainable composition states, $\left[\mathrm{c}_{1}, \mathrm{c}_{2}, \mathrm{c}_{3}\right]$, achievable through steady state designs that have a feed stream of $\mathbf{c}^{\mathbf{f}}$ (Note that $\mathrm{c}_{4}$ is determined by $\left[\mathrm{c}_{1}, \mathrm{c}_{2}, \mathrm{c}_{3}\right]$ through stoichiometry). This problem is also referred to as the 3-dimensional van de Vusse problem.

In Figure (1.1), a set of achievable compositions for the 3-dimensional van de Vusse problem is depicted. The compositions include those that are achievable in the limit for infinite residence times. The boundary of the composition set is made up of 


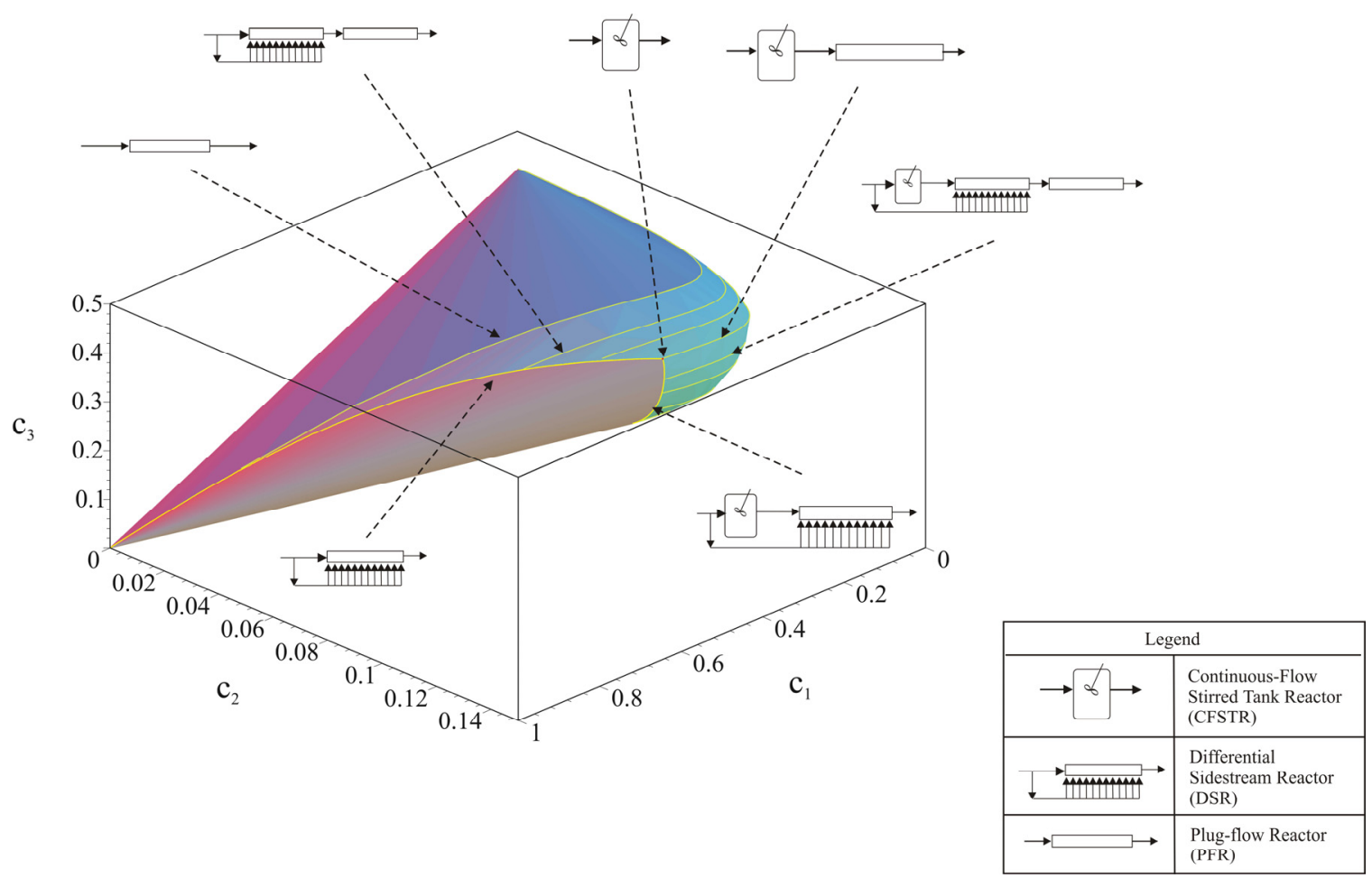

Figure 1.1 Some achievable compositions (in moles per unit volume) for the 3-D Van de Vusse problem

plug-flow reactors (PFRs), continuous-flow stirred tank reactors (CFSTRs) and differential sidestream reactors (DSRs). Differential sidestream reactors are similar to PFRs but in addition they are fed at various points along the length of the reactor. This is only a candidate attainable region and there is no proof as to whether these compositions represent the full set of achievable compositions for this system. Mathematical arguments by Feinberg and Hildebrandt [4] suggest, however, that the boundary of the attainable region will always be shaped by combinations of PFRs, CFSTRs and DSRs. Feinberg has 
also shown how, even when the boundary of the attainable region is unknown, necessary conditions for DSR and CFSTR designs to realize compositions on the boundary of the attainable region can be determined from the reaction kinetics. For CFSTRs [5], specific residence times can be determined for its effluent composition to be an attainable region boundary point. For DSRs [6], special sidestream addition rates can be determined so that its effluent compositions can reside on the boundary of the attainable region. Both of these design criteria provide only necessary conditions for the effluent composition to lie on the boundary of the attainable region; there is no proof that, when these design criteria are followed, the effluent compositions will indeed reside on the boundary of the attainable region.

There are two broad aspects of the attainable region boundary that can be analyzed. Firstly, one is interested in ascertaining what combination of typical reactors like PFRs, CFSTRs and DSRs produce effluents compositions that reside on the boundary of the attainable region. Secondly, one tries to determine the actual location of the boundary of the attainable region.

Knowing what reactor configurations will give effluent compositions on the boundary of the attainable region is very useful, but the recent literature [7] suggests that such designs are unlikely to be implemented. It is known that the DSR trajectories that traverse the boundary of the attainable region must conform to complicated design formulae that become increasingly complex as the number of species increases. Since the design formulae provide only necessary conditions, there is no assurance that if they are followed, compositions on the boundary are truly being traversed by the DSR trajectory. 
On the other hand, knowing the location of the attainable region boundary allows a designer to evaluate how close an existing design is to the limits of what is achievable. Techniques that "build from the inside" are useful because they provide a set of compositions that can be achieved at least through the reactor configurations considered. There is no guarantee, however, that a design that was not taken into consideration could not give a composition set that is larger than the current set believed to be the full set of achievable compositions.

The goal of this thesis is to supplement existing methods that build "from the inside" and not to replace these methods. The idea is to provide kinetic limits on the attainable region by bounding "from the outside." While existing methods strive to ascertain those effluent compositions that are achievable from the feed using constraintconsistent designs, methods described in this thesis strive to elucidate those compositions that are not achievable through any constraint-consistent design whatsoever.

Stated more formally, the goal is to construct a convex bounding set of composition vectors $\mathscr{B}$ such that any composition residing outside $\mathscr{B}$ is not achievable through any constraint-consistent design. If $\mathscr{A} \mathscr{R}$ denotes the true attainable region, then we can see that $\mathscr{A} \subseteq \subseteq \mathscr{B}$. If, through existing methods, one comes up with a set of compositions to create a "candidate" for the attainable region, $\mathscr{C}$, then we can write the following inclusion $\mathscr{C} \subseteq \mathscr{A} \subseteq \subseteq \mathscr{B}$. From this inclusion, it follows that if $\mathscr{C}$ and $\mathscr{B}$ are sufficiently "close" to each other, then one can assert that $\mathscr{B}$ is a sharp outer bound for the true attainable region. It also follows that the design configurations that produce effluent compositions on the boundary of the set $\mathscr{C}$ do indeed traverse close to the limits 
of what is attainable. This novel ability to construct outer bounds on the true attainable region can possibly help researchers to assert that a "candidate" attainable region is truly a good approximation to the actual attainable region.

The assertion that can be made by the outer bounds is stronger than those that can be made by the methods that "build from the inside." The set of achievable compositions obtained by considering known reactor configurations does not preclude the possibility of an unknown design that may enlarge the current set. However, while evaluating an existing design, the outer bounds assert more strongly the fact that no constraint-consistent design can produce a significantly better yield than those compositions within the outer bounds.

In this thesis, we will describe the construction of outer bounds using the "method of bounding hyperplanes." The bounding set so created will be described by a system of linear inequalities of the form,

$$
a_{1}^{L} c_{1}+a_{2}^{L} c_{2}+\ldots \ldots .+a_{N}^{L} c_{N} \leq b^{L} \quad L=1,2, \ldots ., P
$$

where $c_{\mathrm{i}}$ represents the concentration of species $\mathrm{A}_{\mathrm{i}}$ and $\mathrm{P}$ is the number of inequalities making up the polyhedral bound in the space of composition vectors. The number of inequalities , $\mathrm{P}$, corresponds to the number of faces of the polyhedron in $N$-dimensional composition space and roughly depends on how close of an approximation of the outer bounds on the attainable region one wishes to achieve.

Inequalities of the form shown in eqn. (1.2), once they are constructed via theory in this thesis, make the computation of limits on effluent compositions a simple linear programming problem. For example, to find the limiting effluent composition of a certain desired species, one could maximize the concentration of that species subject to 
the constraints in eqn (1.2). The solution to this linear programming problem will then indicate limits on what the designer can ever hope to achieve.

In Chapter 2 of this thesis, we will do a review of relevant literature in the area of process design and process synthesis. Following this, in Chapter 3, we will explain some known ideas and shortcomings about the pure reactor synthesis problem that will motivate our approach. In Chapter 4, we will talk about general bounds on feasible composition space. Chapter 5 will elaborate on the new method of bounding hyperplanes developed as part of this research and in Chapter 6 we will discuss its implementation on a computer. Further, in Chapter 7, we use the method of bounding hyperplanes to study various reaction systems. In Chapter 8 , we conclude with discussions on future research work, theoretical questions and computational challenges. 


\section{CHAPTER 2}

\section{LITERATURE REVIEW}

This chapter will give a brief review of literature in the broad area of process design and synthesis. Literature that is more pertinent to this thesis will be reviewed in the Chapter 3.

\subsection{Process design}

Chemical process design has often been called a form of art [8] where one uses heuristics, knowledge of previous solutions, hypothesizing, numerical computing and then proving experimentally that some new configuration of process equipment will work better than another. A good design slowly evolves over time, through innovation and broadening of the knowledgebase. As a process design task moves further along the design stages it becomes increasing difficult to assess the profitability of design alternatives

Over the past two decades, a new term has dominated process design literature: Process Systems Engineering (PSE) is defined as a systems approach to process design that is complex, ranging from new powerful design methods, to how one 
thinks about the design process itself, to using operation issues in design decision making, to using design simulation methods to aid operations [9].Process synthesis, which will be elaborated upon later, is typically classified as a form of process systems engineering.

One of the intrinsic problems of systems design is knowledge of the complete set of valid chemical process designs (design space) [8] and clear definition of the design goals or constraints. Once these are resolved, relevant designs can be suggested and alternatives may be tested to select an appropriate design. General pedagogical guidelines for performing these tasks can be seen in ref. [10] and more recently in ref. [11].

The design task typically has several objectives: a design must be profitable, reliable, safe, environmentally sound, and each of these objectives affects the other. The economics involved in modifying an existing process dictates that any new design must be better than the existing process design by giving returns on the additional investment and the operating costs [8]. Additionally, the approach taken to achieve the final design is as important as the design itself.

\subsection{Process synthesis in general}

Process synthesis deals with methods to determine the overall structure of a chemical process and the individual components that constitute the process. The individual components are typically well-defined unit operations and the task lies in finding the best configuration from amongst an almost infinite number of alternatives to 
connect these components. James Douglas [12-16] was among the first to suggest a hierarchical decision procedure for process synthesis that was evolutionary in nature and proceeded through a hierarchy of decision levels, where more fine structure was added to the flow sheet at each decision level. Heuristics were used to obtain some of the structural elements of the flow sheet, and other heuristics were used to make some of the decisions required at the various decision levels.

Biegler et al.[10] have suggested the following systematic approaches to process synthesis: total enumeration of all the alternatives in an explicit space, a coordinated search in the space of design decisions, evolutionary methods, superstructure optimization, targeting, problem abstraction, and combinations of these. The attainable regions approach to process synthesis is discussed in detail in Chapter 3.

\subsection{Computational techniques}

Given the task of searching among various alternatives, the power of computing has proven useful to researchers in eliminating alternatives that might not be feasible or those that do not meet the design objectives. Researchers are using many computational techniques to assist the process of evaluating alternatives.

A majority of the computational approaches to process synthesis involve mixed-integer programming techniques. Grossman et al. [17] were among the earliest to suggest mixed-integer linear programming (MILP) in process synthesis. The structural and parameter optimization approach was applied to heat recovery networks [18], utility 
systems [19] and finally total processing systems [20] that combine all the previous systems into a chemical plant. Later focus shifted to using mixed-integer nonlinear programming (MINLP) techniques for global optimization [21]. Raman et al [22, 23] describe a quantitative framework for the integration of logic and heuristic knowledge and converting that into MINLP models for process synthesis. They discuss basic properties of the model formulation and a systematic method to adjust weights for the violation of heuristics and the usage of branch and bound solutions to find a global optimum. Westerberg et al. [24-26] discuss about branch and bound strategies for the synthesis of optimal process flowsheets and separation schemes.

Linke et al. [27] describe the use of stochastic optimization for the synthesis of integrated reaction and separation process superstructures. They claim that this technique enables the systematic exploration of design options hidden in the network formulations. They discuss efficient ways of searching for a globally optimum candidate using two established search techniques: simulated annealing and tabu search.

Yeomans et al. [28] introduced a systematic framework for the representation of superstructures and derivation of optimization models in process synthesis. The two fundamental representations proposed were the state task network (STN) and the state equipment network (SEN) for process systems involving mass, heat, and momentum transfer. The mathematical modeling of the representation was done using generalized disjunctive programming (GDP) followed by systematic conversion into MINLP problems for solving the synthesis of distillation sequences with heat integration. Improvements to the GDP model were later suggested by Lee et al [29-31]. Other methodologies for the optimization of MINLP models were introduced by Adjiman 
et al. [32] such as the special structure mixed nonlinear $\alpha B B(S M N-\alpha B B)$, and the general structure mixed integer nonlinear $\alpha B B(G M I N-\alpha B B)$. The theoretical foundation of these methods provides guarantees that the global optimum solution of MINLPs involving twice-differentiable non-convex functions in the continuous variables can be identified.

In chemical processes with uncertainty, a two-level strategy has been suggested [33-35] for the stochastic synthesis of processes with a fixed degree of flexibility by using the MINLP approach. The nonlinear subproblems were decomposed into the design and operating optimization levels. Dua et al [36-38] describe the use of multiparametric mixed integer nonlinear programming (mp-MINLP) models to solve process synthesis problems under uncertainty. An outer-approximation algorithm is presented for the solution of such mp-MINLPs, described by convex process models, linear in the vectors of binary variables and uncertain parameters. The algorithm follows decomposition principles [39], i.e., constructing a converging sequence of valid upper and lower bounds through the solution of parametric primal and master subproblems.

Achenie et al. [40] suggest a nonlinear programming (NLP) formulation for optimally generating reactor networks that produce the desired effects, given a kinetic mechanism and expressions for the reaction rate, and using the recycle ratio as the determinant of reactor type. The Infinite Dimensional State-Space Approach (IDEAS) is a conceptual framework $[41,42]$ that claims to include all possible process configurations and yields mathematical programs that are convex. The authors claim that this formulation converges to a global optimum but there is no proof as to whether the solution to which convergence has occurred is the true boundary of the attainable region. 
In Hostrup et al. [43], the authors present an integrated approach to the solution of process synthesis, design and analysis problems. Two different process synthesis techniques are integrated: one based on thermodynamic insights and the other based on structural optimization, together with a simulation engine and a properties prediction package.

Some other unique approaches to process synthesis include decisionmapping $[44,45]$ and the graph-theoretic approach $[46,47]$. In the graph-theoretic approach, the maximal structure can be expressed as a process graph and is the union of all combinatorially feasible process structures of a synthesis problem. The authors acknowledge that this is simply a mathematical representation of the "superstructure" used in other methods but the optimal process design is determined through different analysis and algorithms. Researchers at the Technical University of Denmark [48-50] have developed process synthesis techniques based on properties-process relationships. An integrated approach based on the driving forces that promote the desired separation for different separation techniques was developed. These algorithms were combined with a "thermodynamic insights" based process synthesis/design algorithm and an interactive MINLP solution algorithm to find optimal solutions to process synthesis problems.

All of the literature discussed in this section relies on reactor models (otherwise referred to as superstructures) in some way or the other and the general aim is to start with the feed stream and attempt to enlarge the set of achievable compositions until no improvement is seen. The disadvantages of this approach were discussed in chapter 1 and the method described in this thesis serve to complement methods described 
in this section. Our new methods, discussed later in thesis, do not use any reactor models or superstructures.

\subsection{Process synthesis software}

There is a variety of commercial software available that can model entire chemical process design flowsheets and assist designers in evaluating and comparing process designs. The most popular among these are ASPEN, ChemCAD, ProSim and gPROMS.

PROSYN [51, 52] is a user-friendly computer package that uses MINLP based process synthesis techniques. A comprehensive library of models for basic process units and interconnection nodes and a comprehensive library of basic physical properties for the most common chemical components are developed. PROSYN allows one to carry out process synthesis at two basic levels of complexity: MINLP optimization through modeling/decomposition and simultaneous heat integration including Heat Exchanger Networks (HEN) costs. READPERT (reactor development, choice and design expert system) $[53,54]$ is a computer based heuristic-numeric consulting system for the choice of chemical reactors during the first step of process design. On one hand it uses numerical calculations and on the other it uses expert system technology and knowledge representation forms like rules and frames. READPERT can be used as a stand-alone system as well as with PROSYN.

"Jacaranda" [55-58] is an automated process synthesis package that can be used to aid an engineer in making decisions in the early stages of process plant design. It is part of an object oriented framework written in the Java language. In order to search 
the solution space, variables such as pressures and component flow rates are mapped to discrete levels. Interval analysis is used to bound the possible values of discretized variables to give bounded and globally optimal solutions. Interval arithmetic was used to bound the possible costs of unit designs and the list of best solution structure was determined for capital, operating and annualized cost criteria.

The software described in this section either aid a designer to manually evaluate process design alternatives or perform "automated" process synthesis. Nevertheless, these software rely on methods described in the previous section. One could begin by defining a feed stream in the software interface, define the reactions that occur and continually add various reactors and mixers in every imaginable configuration possible and attempt to enlarge the set of compositions that can be achieved through the designs considered. There is always the possibility that a novel reactor, not present in the repository of reactor models available in the software, might in fact enlarge what the designer considers to be the full set of achievable compositions based on the simulations. 


\subsection{Reactor synthesis with and without separators}

Given a chemical reaction network with kinetics and a process objective, a designer sets out to design the optimal reactor configuration that satisfies design constraints. Among the earliest work in this area by Chitra et al. [59,60], researchers proposed optimizing superstructures of reactors containing both stirred tank and plug flow reactors in all possible configurations. This approach, however, was plagued by the existence of local optima during the optimization process [8].

Nisoli et al. [61] studied nonhomogeneous reaction systems with separation by applying the "attainable region" approach for reaction-mixing systems combined with geometric methods for the feasibility of separations. An activity-based formulation for both nonideal vapor-liquid equilibrium (VLE) and reaction-rate expressions was applied to develop hybrid reactor-separator models for the multiphase continuous stirred tank reactor (CSTR) and plug flow reactor (PFR) with simultaneous vapor removal. The technique was demonstrated on two nonideal ternary mixtures: the production of dimethyl ether by dehydration of methanol, and the production of methyl tert-butyl ether from isobutene and methanol.

In ref. [62] , Nicol et al. use an "attainable region technique" to find the optimum process design for an exothermic reversible reaction system where allowance was made for external cooling using two different constant temperature utilities. Rooney et al. [63] present a hybrid approach using both mathematical programming methods and attainable region concepts to extend reactor network synthesis techniques to include model parameter uncertainty. Multiperiod optimization connects are used to extend the 
MINLP model to include model parameter uncertainty. In Lakshmanan et al. [64] a reactor network synthesis algorithm, which is an MINLP formulation consistent with geometric attainable region concepts, was integrated with mass exchange network concepts. A reaction-mixing-separation model was developed and solved with other process constraints to find the optimal process design. Lakshmanan et al [65] used attainable-region properties to design a fixed number of reactor modules for reactor network synthesis. The reactor modules, made up of PFRs, CSTRs and DSRs, are used to synthesize the optimal reactor configuration with respect to a specific objective function and the problem is formulated as a compact mixed integer nonlinear program. Pahor et al.[66-68] suggest another MINLP approach to the synthesis of reactor networks in an equation-oriented environment. Their model comprises a general superstructure in which several reactor systems such as pure CSTR, pure plug flow reactor, pure recycle reactor, cross-flow reactors and various combinations thereof. Schweiger and Floudas [69] present a framework for the synthesis of optimal chemical reactor networks wherein the feasible design alternatives are represented using a process superstructure that includes continuous stirred-tank reactors and cross-flow reactors along with mixers and splitters that connect the units. The superstructure was mathematically modeled using differential and algebraic constraints and the resulting problem was formulated as an optimal control problem. Friedler et al. [44] present a technique derived from the mathematical notion of decision-mapping to make consistent and complete decisions in process design and synthesis. Mathematical properties of decision-mapping were described using settheoretic formalism and the efficacy of the technique was shown by synthesizing a reactor-network. 
The reviews discussed in this section show how all the reactor synthesis approaches use typical reactor models and variations of these models in creating an optimal reactor or reactor-separator configuration to achieve process goals. Methods developed in this thesis will not rely on any such reactor models.

\subsection{Industrial applications}

Due to the large number of design objectives in industrial problems, process synthesis techniques have only recently been successful in industrial applications. In Linke et al. [70] a generic process synthesis and an optimization tool suitable to take up applications in the design of waste treatment systems and in pollution prevention is presented. Applications of the synthesis framework to waste water treatments processes using activated sludge and to natural gas sweetening in hybrid membrane-adsorption processes are discussed. Similar technology is used in Uppaluri et al. [71] to develop robustly optimal process designs for gas permeation membrane and hybrid membrane systems used in the separation of air, up-gradation of a lean hydrogen hydro-cracking refinery stream and acid gas removal from natural gas streams using membrane and fixed bed adsorption networks.

Nisoli et al. [72] have studied feasible regions for step-growth melt polycondensation systems important in the production of Nylon 6,6. They use an attainable-region approach for reaction, mixing, and separation to study the step-growth melt polycondensations. Smith et al. $[73,74]$ attempt to find feasible compositions for random copolymerization and other polymerization reaction systems. Milne et al. [75] apply their attainable region concepts to the oxidative dehydrogenation of 1-Butene in 
inert porous membrane reactors in the manufacture of butadiene. Kauchali et al. [76] attempt to find a candidate attainable region for the water-gas shift (WGS) reaction under adiabatic conditions. Lakshmanan et al. $[77,78]$ use MINLP strategies to find an optimal reactor network that improves the conversion of ethylene to vinyl chloride and minimizes the formation of byproducts during the production of vinyl chloride monomer. Steffens et al. $[79,80]$ describe how process synthesis techniques were used to evaluate bioprocess designs. Physical property information is used to screen candidate units, thereby reducing the size of the synthesis problem. Due to the large number of components and wide range of unit operations available, only units that exploit large property differences between components in a stream are selected. An implicit enumeration algorithm incorporates these ideas and is implemented in software that generates a ranked list of flowsheets which is subsequently analyzed in more detail. Two case studies were presented in Steffens et al. [80] : the first was a process to synthesize a downstream purification process for a protein secreted from S. cervisae; the second one involved the purification of bovine somatotropin (BST), a product which forms inclusion bodies within the E.Coli host cells.

The industrial examples described in this section use techniques that are specific to the problem being resolved. They are not easily applicable to other process systems. The method of bounding hyperplanes to be introduced in this thesis is broad and can be applied to any process system that satisfies the design conditions for which these have been derived. 


\section{CHAPTER 3}

\section{THE ATTAINABLE REGION APPROACH TO THE}

\section{PURE-REACTOR SYNTHESIS PROBLEM AND ITS SHORTCOMINGS}

\subsection{Comparing the pure-reactor and the reactor-separator synthesis problems}

Two broad classes of design have emerged as dominant focuses in the attainable region approach to process synthesis. These are the pure-reactor synthesis problem and the reactor-separator synthesis problem [7]. The feed to the system is specified and the chemical reaction network with kinetics is presumed to be known completely. In the pure-reactor synthesis problem, one seeks to find out the complete set of output compositions achievable through the given feed in steady-state designs using reaction and mixing alone. In the reactor-separator synthesis problem, the goal is to find limits on the species production rates achievable through steady-state designs involving reaction, mixing and separations.

It is obvious that the pure reactor synthesis problem is the less compelling of the two problems. Separators can definitely help in giving a better productivity of the desired species and therefore why would the designer not invoke separators in a process design? The pure-reactor synthesis problem has the merit of being able to give 
information on what reactor configuration can perhaps minimize the formation of undesired species. Separation of the undesired species from the desired species becomes less arduous when its formation can be suppressed by an intelligent reactor configuration design. The pure-reactor design can be considered as a special case of the reactorseparator design and therefore the bounds on compositions that can be attained by reactor-separator designs also serve in bounding those compositions that can be attained by designs permitting reactions and mixing alone. This idea will be discussed further in Chapter 4.

For the reactor-separator synthesis problem, the CFSTR Equivalence Principle [81] gives guidance on how one can compute the outer bounds on productivity for a reactor-separator system in which the reactor components have a specified capacity and are constrained to operate within specified composition-temperature limits. The bounds computed in this manner are exact to the extent that one can realize arbitrary separations.

For the pure-reactor synthesis problem, the outlook is less promising [7]. On the positive side, detailed statements [4-6] can be made about the design of reactors that shape the boundary of the attainable region on the basis of the kinetic rate laws and available feed stream compositions, even when the (location of the) boundary of the attainable region remains unknown. On the negative side is the reality that these "critical" designs that shape the boundary of the attainable region depend in a very complicated manner on the rate laws and higher derivatives of these rate laws (depending on the number of reactions). This complexity is intrinsic $[6,7]$ to the reactor synthesis problem and is not due to the approach used to solve the problem. 


\subsection{The pure-reactor synthesis problem}

In this problem, we consider the following question: What is the full set of mixture compositions achievable from prescribed feed streams by using solely steadystate designs that involve chemical reactions and bulk mixing?

Consider a system with $\mathrm{N}$ species, say $\mathrm{A}_{1}, \mathrm{~A}_{2} \ldots . \mathrm{A}_{\mathrm{N}}$, among which transformations take place due to several chemical reactions. The systems described here are restricted to isothermal, homogeneous systems with fixed density everywhere in the mixture. The composition anywhere in the system may be represented by the vector $\mathbf{c}=\left[c_{1}, c_{2}, \ldots c_{\mathrm{N}}\right]$ in $\mathbb{R}^{N}$, where $c_{I}$ is the molar concentration of species $\mathrm{A}_{\mathrm{I}}$ and $\mathbb{R}^{N}$ is a vector space of $\mathrm{N}$-tuples of real numbers.

The species-formation-rate function, denoted by $\mathbf{r}(\cdot)$, derives from the kinetics for a given reaction network. If the local composition somewhere in the mixture is represented by the composition vector $\mathbf{c}$ then one can write the vector,

$$
\mathbf{r}(\mathbf{c})=\left[\mathrm{r}_{1}(\mathbf{c}), \mathrm{r}_{2}(\mathbf{c}), \ldots . ., \mathrm{r}_{N}(\mathbf{c})\right],
$$

where $\mathrm{r}_{j}(\mathbf{c})$ is the rate at which species $\mathrm{A}_{j}$ is being produced in the mixture due to the occurrence of all chemical reactions.

The problem question can be restated from a geometric viewpoint[7]:

What is the full set of composition vectors in $\mathbb{R}^{N}$ attainable from feed stream(s) of prescribed compositions $\mathbf{c}_{1}^{\mathrm{f}}, \mathbf{c}_{2}^{\mathrm{f}}, \ldots \mathbf{c}_{K}^{\mathrm{f}}$ using steady-state isothermal designs involving reactions and mixing alone. This set is called the attainable region and is abbreviated as $\mathbf{A R}$ for the purpose of this discussion. It is trivial to note that $\mathbf{A R}$ will contain the vectors 
$\mathbf{c}_{1}^{\mathrm{f}}, \mathbf{c}_{2}^{\mathrm{f}}, \ldots \mathbf{c}_{K}^{\mathrm{f}}$, but it will always be larger. A typical design would be to use a steady-state plug flow reactor (PFR) with feed, say, $\mathbf{c}_{1}^{\mathrm{f}}$ to give a set of compositions (typically a curve in $\mathbb{R}^{N}$ ) for different lengths of the PFR. Similarly, another design could use a steadystate continuous flow stirred tank reactors (CFSTR) with feed, say, $\mathbf{c}_{1}^{\mathrm{f}}$ to produce another set of effluent compositions for various residence times in the CFSTR. Figure 3.1 shows how one could chose from a large number of possible reactor configurations.

If $\mathbf{c}^{\#}$ is a composition on the PFR trajectory and if $\mathbf{c}^{\# \#}$ is a composition on the CFSTR locus, then any composition on the line joining $\mathbf{c}^{\#}$ and $\mathbf{c}^{\#}$ in $\mathbb{R}^{N}$ can be achieved by mixing effluent streams that contain these two compositions in varying proportions. Any composition lying in the interior of the polyhedron (in $\mathbb{R}^{N}$ ) with vertices taken from $\mathbf{c}^{\#}, \mathbf{c}^{\# \#}, \mathbf{c}_{1}^{\mathrm{f}}, \mathbf{c}_{2}^{\mathrm{f}}, \ldots \mathbf{c}_{K}^{\mathrm{f}}$ can be attained by bulk mixing of the appropriate streams. Reactors may be connected in various configurations such that the effluent of one reactor acts as the feed for the other and new compositions can be attained in this manner.

The attainable region is [7] the full set in $\mathbb{R}^{N}$ of compositions that might be realized from the primary feed streams by all such processes (and also compositions that might not be realizable but that can be realized arbitrarily closely). A frequently used technique to find a set of realizable compositions starts with primary feed streams and then considers various reactor types and interconnected configurations in an attempt to enlarge the set of compositions that are realizable. This technique does not guarantee that the full set of realizable compositions has been found, even when no further enlargement of the set seems possible through the reactor configurations considered. 


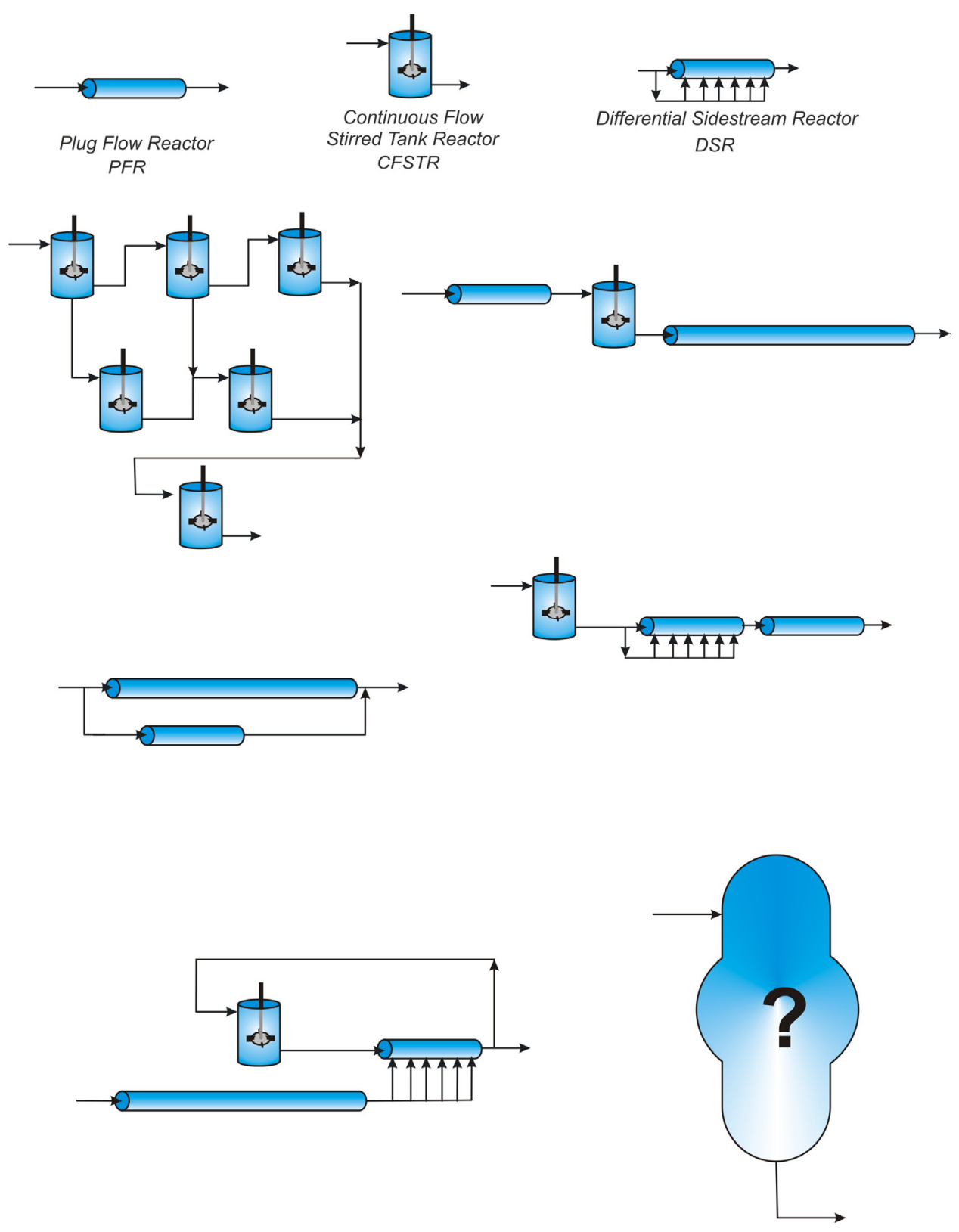

Figure 3.1 Possible reactor configurations for the pure reactor synthesis problem 
Feinberg and Hildebrandt[4] have shown that conventional reactors PFRs, CFSTRs and DSRs will shape the attainable region's boundary with each of these reactor types playing specific roles in shaping the boundary. Figure 1.1 shows those compositions that are achievable through the designs described the authors.

The derivation of the design formulae are beyond the scope of this thesis, so we provide just a brief overview of the results to motivate and also serve as a comparison for the new method described in later chapters of this thesis.

\subsection{Universal properties of the attainable region}

An extreme point of a closed convex set (in $\mathbb{R}^{N}$ ) is defined as a member of the set that does not lie in the interior of a line segment contained in the set. The boundary of a closed convex set is thus made up of extreme points and straight line segments. It has been shown in the literature [4] that the attainable region is also a closed convex set and that its boundary will be made up of the union of manifolds of extreme points and straight line segments which will be elaborated in this section. 


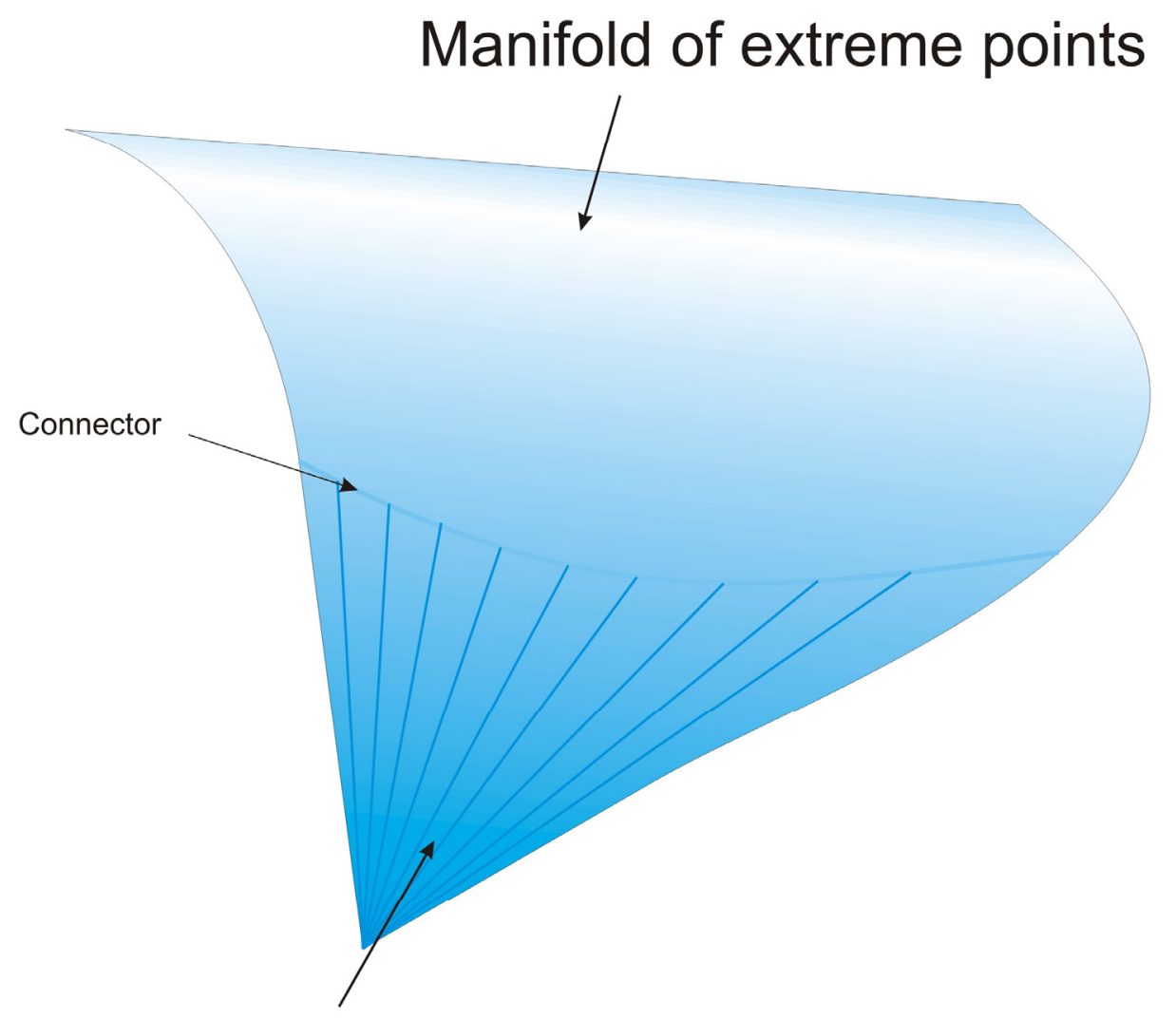

Straight line section

Figure 3.2 Manifold of extreme points meeting a straight line section smoothly

Figure 3.2 shows a possible fragment that could reside on the boundary of a closed convex set in $\mathbb{R}^{3}$. (The discussion in this section will mostly make references to assertions made in $\mathbb{R}^{3}$ but the general assertions will extend to higher dimensions as well.) The figure shows a straight line segment meeting a two-dimensional manifold of extreme points "smoothly" along a one-dimensional manifold of extreme points[4]. In $\mathbb{R}^{3}$, the one-dimensional manifold is typically a curve. 


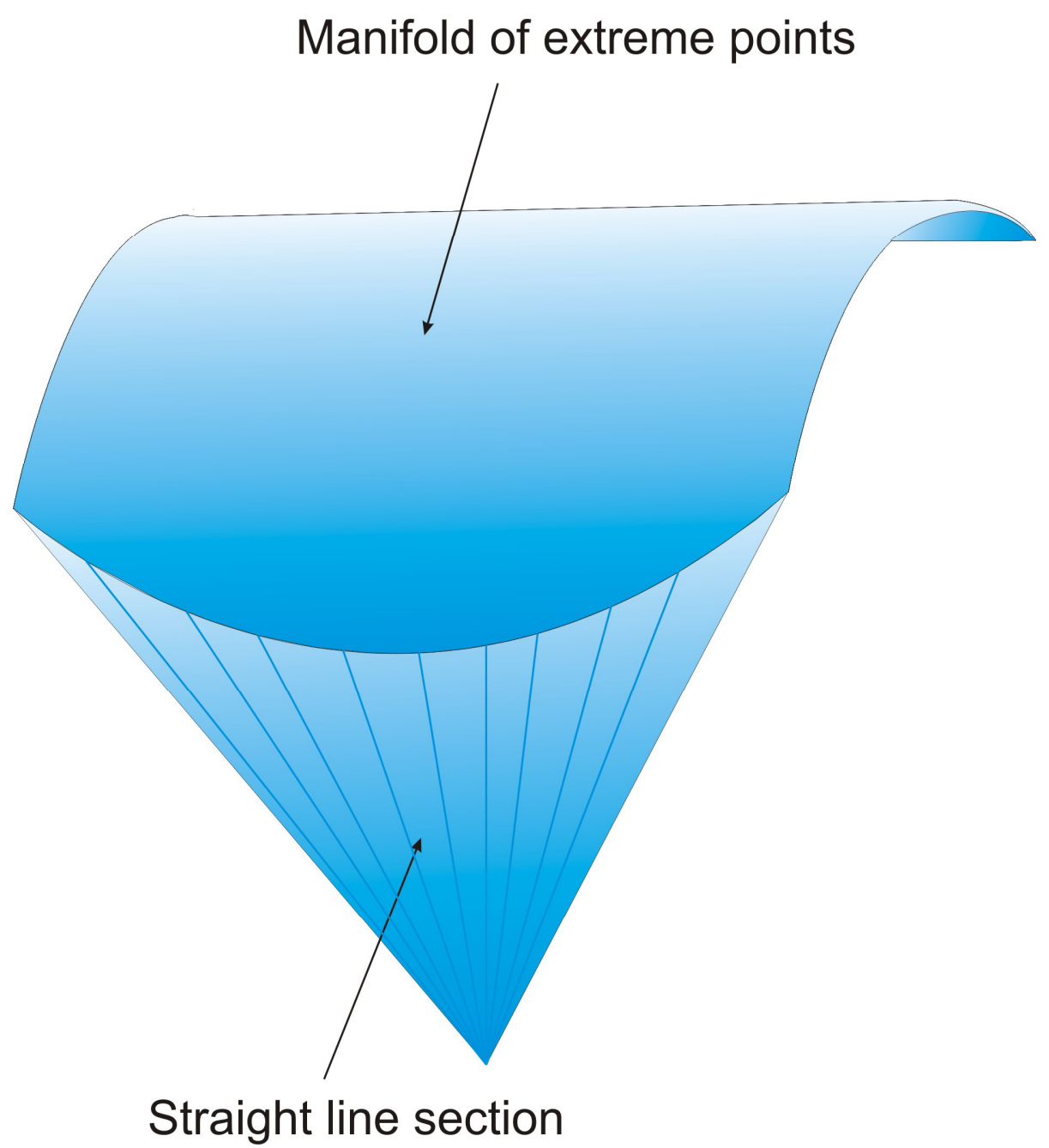

Figure 3.3 Manifold of extreme points meeting a straight line section roughly

Figure 3.3 shows another possible fragment that could represent the boundary of a closed convex set in $\mathbb{R}^{3}$. The figure shows a straight line segment meeting a twodimensional manifold of extreme points "roughly" along a one-dimensional manifold of extreme points [4]. 


\section{PFR Trajectories}

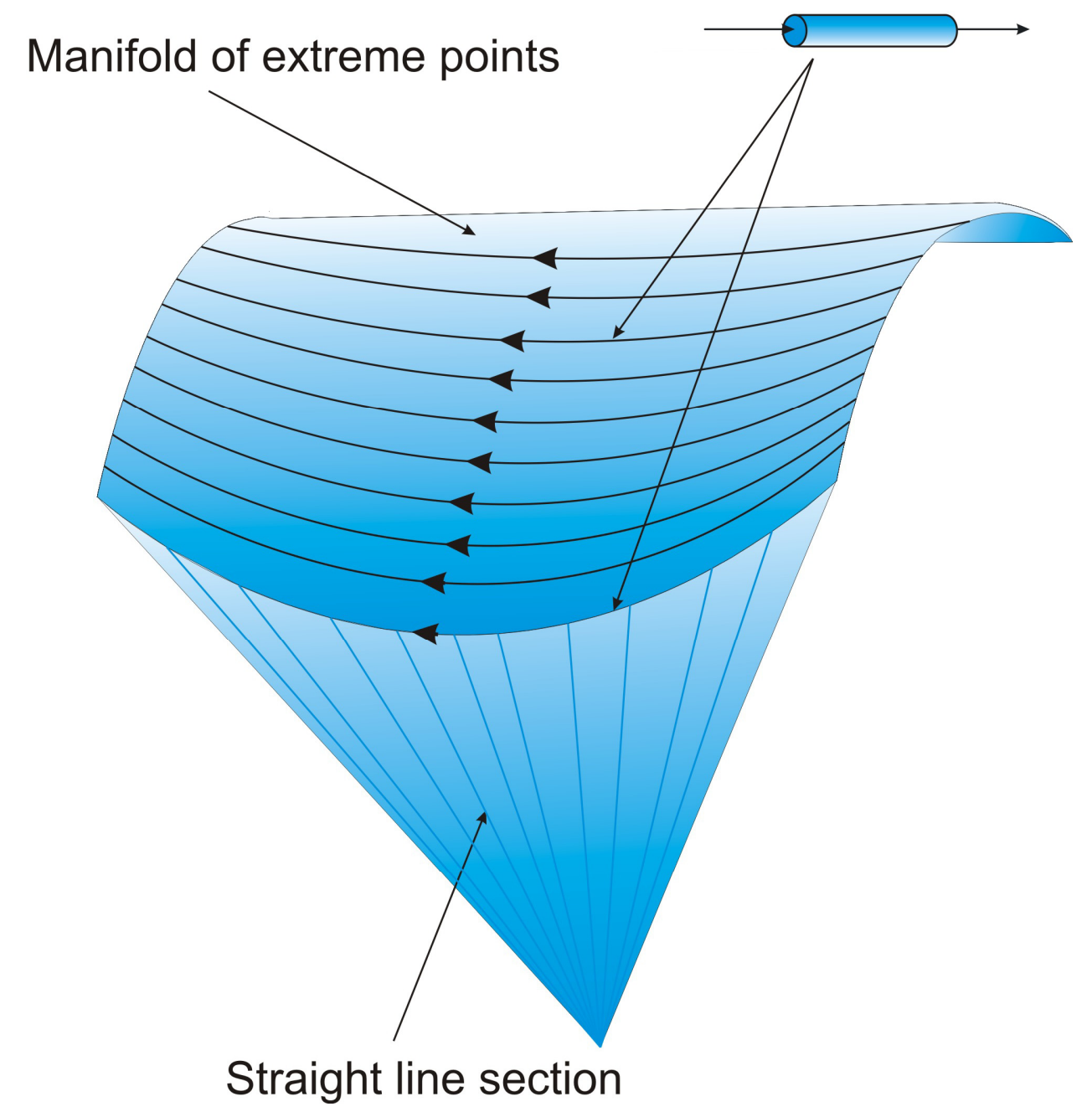

Figure 3.4 PFR composition trajectories that reside on the manifold of extreme points

Feinberg and Hildebrandt [4] have shown that on the boundary of the attainable region, manifolds of extreme points are made up entirely of plug-flow reactor trajectories. Such manifolds are called "protrusions." When a protrusion meets a straight line section "roughly", then the edge at which the two sections join is again the union of PFR composition trajectories. Hence, if the fragment shown in Figure 3.3 was on the boundary of the attainable region for a given chemistry, then the two dimensional 
protrusions must comprise of PFR composition trajectories as shown in Figure 3.4. Note also that the curve along which the straight line section meets the protrusion is also a PFR composition trajectory.

Feinberg and Hildebrandt [4] describe a different situation when the straight-line section meets the protrusion smoothly as in Figure 3.2. A connector on the boundary of the attainable region is defined as the edge between a straight line section and a protrusion at which the two sections join smoothly and at which the PFR composition trajectories that traverse the protrusion point away from the straight line section. It has been shown [4] that a connector is necessarily the union of DSR composition trajectories and CFSTR operating points, whose feed streams have compositions from among those already within the attainable region.

Thus, if the fragment shown in Figure 3.3 were part of the attainable region for a reaction system, then the reactors that might constitute the fragment would look like those shown in Figure 3.5. In the figure, the connector is made up of the union of two DSR trajectories and a CFSTR effluent composition $\mathbf{c}^{*}$. The figure also illustrates earlier assertions about how typical reactor types might shape the boundary of the attainable region. The PFR composition trajectories point outward from the connector and traverse the protrusions on the boundary of the attainable region and in that sense form a system of "highways" [7] along which the extreme points on the boundary may be accessed. The DSRs and CFSTRs provide access to the connectors of straight-line segments and protrusions such that a desired PFR trajectory traversing on the protrusion may be accessed. 


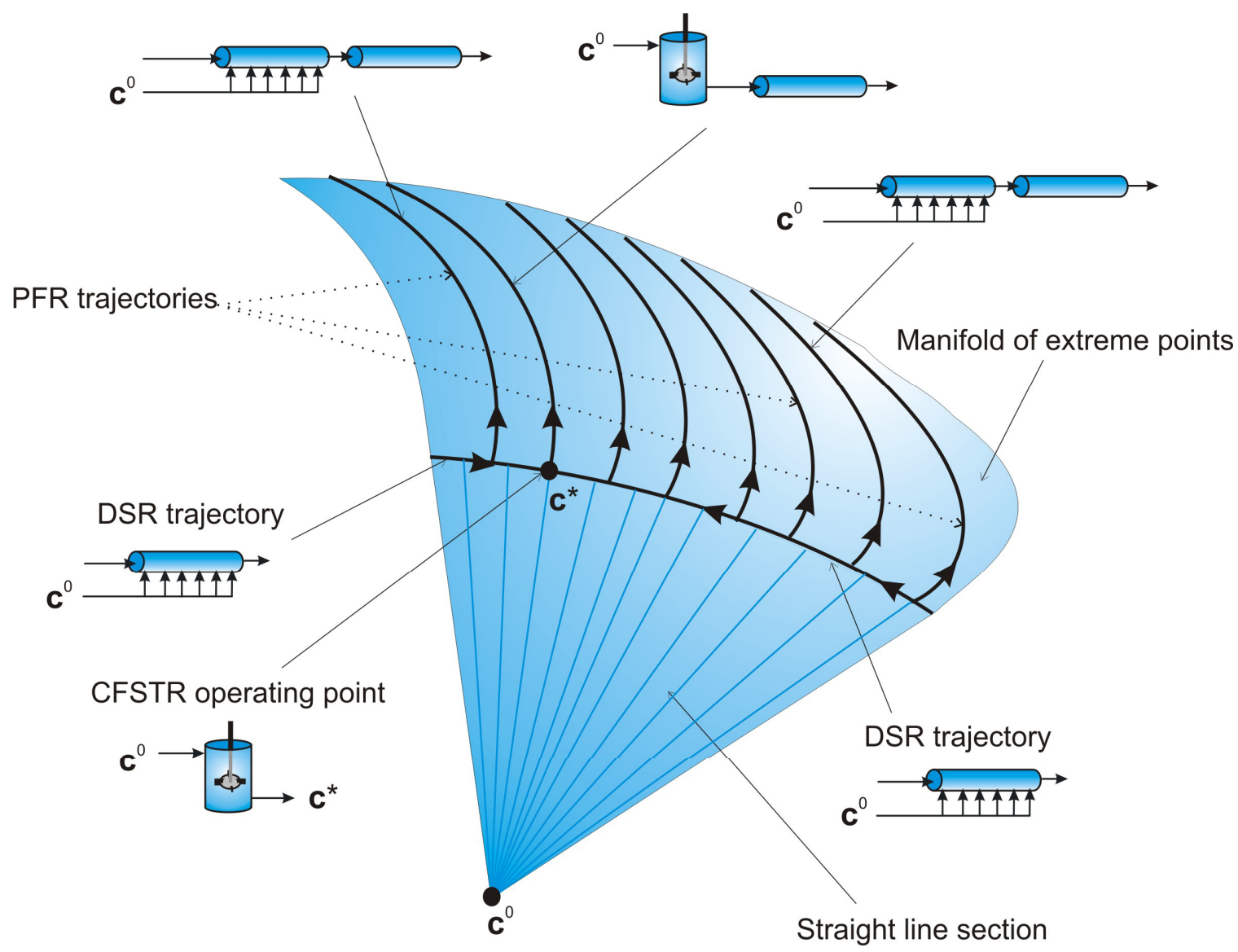

Figure 3.5 Reactor configurations that shape the boundary of the attainable region where the smooth-line section meets the protrusion smoothly

\subsection{Critical CFSTRs}

Consider a CFSTR as shown in Figure 3.5 with a feed stream of composition $\mathbf{c}^{0}$. Suppose also that the effluent stream has a composition $\mathbf{c}^{*}$ such that $\mathbf{c}^{*}$ is an extreme point residing on the boundary of a candidate attainable region.

Critical CFSTRs are special CFSTR designs satisfying necessary conditions such that it can produce an effluent composition(s) residing on the boundary 
of the attainable region. Given a chemical reaction network with kinetics, it has been shown[5] that only certain exceptional residence times give an effluent composition that can be an extreme point residing on the boundary of the attainable region. These special residence times and effluent compositions can be calculated from the kinetics and $\mathbf{c}^{0}$ even when the boundary of the attainable region is not known. Feinberg [5] describes that for a given species-formation-rate function there might exist a set of compositions that can simultaneously be an extreme point of some attainable region and the effluent composition of a steady-state CFSTR.

The design of critical CFSTRs can be demonstrated through an example using the Van de Vusse reaction system as shown in eqn (3.1)

$$
\begin{aligned}
& \mathrm{A}_{1} \stackrel{1}{\rightarrow} \mathrm{A}_{2} \stackrel{k}{\rightarrow} \mathrm{A}_{4} \text {. } \\
& 2 \mathrm{~A}_{1} \stackrel{k^{\prime}}{\rightarrow} \mathrm{A}_{3}
\end{aligned}
$$

The reaction kinetics is assumed to be mass-action and the rate constants are as shown. We are interested in finding out if a CFSTR with a feed (and attainable) composition $\mathbf{c}^{0}=[1,0,0,0]$ can be designed such that its effluent composition is an extreme point of an attainable region that arises from some specified set of primary feed streams. In ref [5], it was shown that such a CFSTR would have a residence time, $\theta$ that bears a strict relationship to the rate constants as shown in eqn (3.2).

$$
\theta=\frac{1-\sqrt{\frac{k}{2 k^{\prime}}}}{k+\sqrt{\frac{k}{2 k^{\prime}}}} .
$$

Since residence times are nonnegative, we get the requirement that $k \leq 2 k^{\prime}$. This also implies that if $k>2 k^{\prime}$, then one cannot design a CFSTR whose feed composition is 
$\mathbf{c}^{0}=[1,0,0,0]$ and whose effluent composition is an attainable region extreme point. If $k<2 k^{\prime}$, then one can calculate exactly one positive residence time such that the CFSTR effluent composition would be an attainable region extreme point (This residence time can be calculated from equation (3.2)).

Typically, the Van de Vusse reaction has been studied with the rate constants fixed at $k=1$ and $k^{\prime}=10$. For these rate constants and a feed composition of $\mathbf{c}^{0}=[1,0,0,0]$, there exists an exceptional CFSTR residence time $\theta=0.6345$ (see ref. [7]) for which the effluent composition might be an attainable region extreme point. While making such an assessment, even knowledge of the primary feeds from which the attainable region has been derived, is not necessary.

The mathematical framework developed to describe the nature of critical CFSTRs is not limited to small systems like Van de Vusse but have been applied to larger systems as well. For example, in ref [5] a system with six species and four reversible reactions is found to have exactly two positive CFSTR residence times for which the effluent composition could be an attainable region extreme point. As shown in Figure 3.4, critical CFSTR operating points serve as rest points[7] associated with the DSR trajectories. From the theory suggested in [5], it can be asserted that DSRs rather than CFSTRs impart the primary shape of connectors on the boundary of the attainable region. This then leads us to the importance of critical DSRs and their implications in trying to ascertain the boundary of the attainable region for the pure reactor synthesis problem. 


\subsection{Critical DSRs}

Figure 3.6 depicts a differential side-stream reactor (DSR) operating at steady state. The composition of the feed at the one end of the DSR is $\mathbf{c}^{*}$ and the composition of the sidestream is fixed at $\mathbf{c}^{0}$ but the addition rate of this stream varies

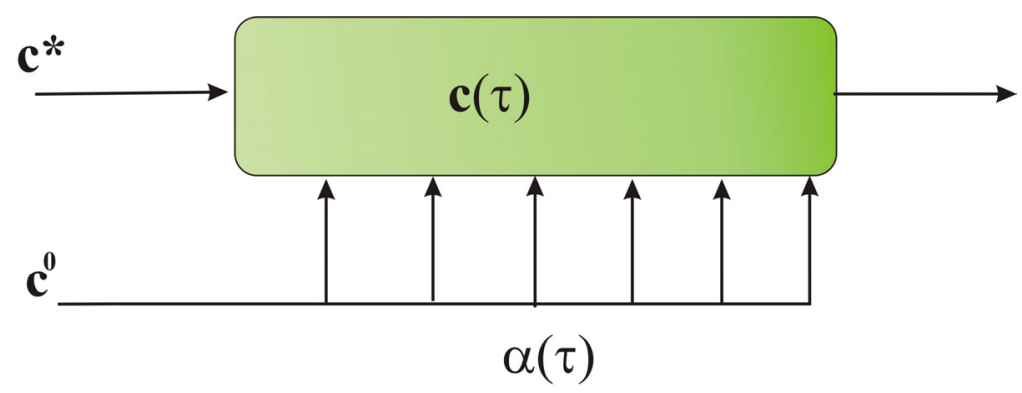

Figure 3.6 Schematic of a DSR

axially along the length of the reactor. The local sidestream addition rate is given by $\alpha(\tau)$, which is the volumetric rate of sidestream added per unit reactor volume, and $\tau$ denotes the local residence time $[4,7]$. Since we are dealing with a steady state reactor, one can describe an addition rate law [7] where the local addition rate along the length of the reactor is tuned to the local steady state composition inside the reactor.

Feinberg [6] has shown that, for a DSR trajectory to consist entirely of attainable region extreme points, it is necessary that the sidestream addition rate policy conform to detailed design equations that are derived from $\mathbf{r}(\cdot)$ (the species-formationrate function) and $\mathbf{c}^{0}$. Even when the boundary of the attainable region is unknown, design equations for these critical DSRs can be formulated. Examples showing how one 
could compute these design equations are shown in ref [6] but they reveal a disturbing fact: for relatively simple reaction networks the addition rate policy is extremely complicated. For reaction networks with a larger number of species and more reactions, the rate policy gets more complicated whereas the fact remains that the addition rate policy computed is the only one that could serve to shape the attainable region's boundary.

\subsection{Analyzing the "critical" design equations}

One of the goals in finding the attainable region is that the designer would like to know what designs might be implemented to achieve those compositions that constitute the outermost limits of what is achievable[7]. As described in the previous sections, precise statements can be made about those designs that shape the boundary of the (unknown) attainable region.

The critical DSRs that shape the boundary of the attainable region reveal a negative outlook from the perspective of practical implementation. In ref [6], one can see how even for a simple four-reaction network the compulsory addition rate policy is highly complex. For a reaction network with five linearly independent reactions, the sidestream addition rate is governed by differential equations involving polynomials in the species concentrations that can have hundreds of terms[6]. For a system with six independent reactions, the sidestream addition rate policy is made up of differential equations that fill up an entire page[6]. 
Feinberg [6] has shown that as the number of independent reactions

increase, increasingly higher order derivatives of $\mathbf{r}(\cdot)$, the species-formation-rate function, appear in the addition rate policy for the critical DSRs. This dependency on the species formation rate function has dire consequences from a real-life application perspective because the rate functions typically derive from kinetic measurements that are inherently uncertain. The rate functions derived in this manner are often an approximation of the true kinetics of the system. The inherent uncertainty in the reaction kinetics is magnified in the higher derivatives of the rate function especially those that appear in the sidestream-addition-rate policy equations. Designs that try to implement such complex rate policy equations might turn out to be futile due to the inherent uncertainty in addition to the complex nature of the addition-rate-policy equations themselves. One could account for such uncertainty through some mathematical framework but the complexity of the sidestream-addition rates are an inevitable reality of the analysis and not due to a flawed approach. Moreover, these complex formulas provide only necessary conditions and hence it remains difficult to know which designs actually do shape the boundary of the attainable region.

In this chapter we have reviewed known theories about the attainable region for the pure-reactor synthesis problem. Analysis of its shortcomings suggest that trying to find the actual designs that traverse the true boundary might be futile. Using this as motivation, this thesis focuses on trying to find an approximate location for the true boundary of the attainable region rather than the exact designs that might shape the boundary for reasons mentioned in this section. 


\section{CHAPTER 4}

\section{GENERAL BOUNDS ON FEASIBLE COMPOSITION SPACE}

The aim of this chapter is to review methods that can be used to compute bounds on product yield compositions for a given chemical reaction network. We introduce mathematical preliminaries relevant to this chapter in Section 4.1. Section 4.2 will deal with the most commonly used bounds based on stoichiometry of the reaction network. Section 4.3 will deal with a less frequently used idea based on thermodynamics. Section 4.4 will describe a novel method for computing bounds based on concepts derived from the attainable region theory for the reactor-separator synthesis problem.

\subsection{Mathematical preliminaries}

Let us denote a vector space of $N$-tuples of real numbers by $\mathbb{R}^{N}$. We recall the following definitions from linear algebra $[83,90]$ :

Linear subspace: A set $\boldsymbol{U}$ of vectors in $\mathbb{R}^{N}$ is a linear subspace if (i) whenever $\mathbf{x}$ and $\mathbf{y}$ are in $\boldsymbol{U}$, then $(\mathbf{x}+\mathbf{y})$ is also in $\boldsymbol{U}$, and (2) whenever $\mathbf{x}$ is in $\boldsymbol{U}, \alpha$ is a number, then $\alpha \mathbf{x}$ 
is also in $\boldsymbol{U}$. A set of vectors $\left\{\mathbf{X}_{1}, \mathbf{X}_{2}, \ldots . ., \mathbf{X}_{k}\right\}$ in $\mathbb{R}^{N}$ is linearly dependent if there are numbers $\alpha_{1}, \alpha_{2}, \ldots ., \alpha_{k}$ at least one of which is not zero such that,

$$
\alpha_{1} \mathbf{X}_{1}+\alpha_{2} \mathbf{X}_{2}+\ldots \ldots+\alpha_{k} \mathbf{X}_{k}=\mathbf{0}
$$

Otherwise $\left\{\mathbf{X}_{1}, \mathbf{X}_{2}, \ldots . . \mathbf{X}_{k}\right\}$ is linearly independent. A linear subspace $\Omega \subset \mathbb{R}^{N}$ is said to be of dimension $m$ if there exists in $\Omega$ a linearly independent set containing $m$ vectors but no linearly independent set containing $m+1$ vectors. If $\Omega$ is of dimension $m$ then any linearly independent set in $\Omega$ containing $m$ vectors is called a basis for $\Omega$.

Span: Given any set of vectors $\left\{\mathbf{X}_{1}, \mathbf{X}_{2}, \ldots ., \mathbf{X}_{k}\right\}$ in $\mathbb{R}^{N}$, we say that a vector $\mathbf{v}$ lies in the span of the set of vectors $\left\{\mathbf{X}_{1}, \mathbf{X}_{2}, \ldots ., \mathbf{X}_{k}\right\}$ if it can be expressed as a linear combination of those vectors. That is, we will have:

$$
\mathbf{v}=\alpha_{1} \mathbf{X}_{1}+\alpha_{2} \mathbf{X}_{2}+\ldots \ldots+\alpha_{k} \mathbf{X}_{k}
$$

where $\alpha_{1}, \alpha_{2}, \ldots . ., \alpha_{k}$ is any choice of real numbers.

Parallels of a linear subspace: Let $\Omega$ be a linear subspace of $\mathbb{R}^{N}$ and let a be some fixed vector in $\mathbb{R}^{N}$ (not necessarily in $\Omega$ ). By $\mathbf{a}+\Omega$ we refer to the set in $\mathbb{R}^{N}$ obtained by adding a to every vector of $\Omega$. In other words, we can write

$$
\boldsymbol{W}=\mathbf{a}+\Omega=\left\{\mathbf{X} \in \mathbb{R}^{N}: \mathbf{X}=\mathbf{a}+\omega, \text { where } \omega \in \Omega\right\}
$$

where $\boldsymbol{W}$ is called an affine subspace and we can say that [6] $\mathcal{W}$ is a parallel of $\Omega$ translated by a .The following statements are true about parallels. If $\Omega$ is a linear subspace of $\mathbb{R}^{N}$, then (1) Two vectors of $\mathbb{R}^{N}$ lie in the same parallel of $\Omega$ if and only if 
their difference lies in $\Omega$, (2) If $\mathbf{b}$ lies in $\mathbf{a}+\Omega$ then $\mathbf{b}+\Omega$ is the same as $\mathbf{a}+\Omega$, and (3) Every vector of $\mathbb{R}^{N}$ lies in one and only one parallel of $\Omega$

Convex hull: Let $V$ be a real finite-dimensional vector space that contain the set of vectors $\left\{\mathbf{x}_{1}, \mathbf{x}_{2}, \ldots \ldots, \mathbf{x}_{k}\right\}$. We can say that a vector $\mathbf{v}$ in $V$ is a convex combination of $\left\{\mathbf{x}_{1}, \mathbf{x}_{2}, \ldots \ldots, \mathbf{x}_{k}\right\}$ if there exists a set of numbers $\left\{\lambda_{1}, \lambda_{2}, \ldots \ldots ., \lambda_{k}\right\}$ such that

$$
\begin{gathered}
\lambda_{i} \geq 0, \quad i=1,2, \ldots, k, \\
\sum_{i=1}^{k} \lambda_{i}=1,
\end{gathered}
$$

and

$$
\mathbf{v}=\sum_{i=1}^{k} \lambda_{i} \mathbf{x}_{i}
$$

If $\mathbf{v}$ and $\mathbf{w}$ are distinct vectors in $V$, then a line segment that connects $\mathbf{v}$ and $\mathbf{w}$ is the set of all vectors in $V$ that reside in the set given by

$$
\{\lambda \mathbf{v}+(1-\lambda) \mathbf{w} ; 0 \leq \lambda \leq 1\}
$$

In other words, the line segment that connects $\mathbf{v}$ and $\mathbf{w}$ is the set of all convex combinations of $\mathbf{v}$ and $\mathbf{w}$. The interior of the line segment refers to the set of points corresponding to $0<\lambda<1$.

Let $\mathscr{A}$ be a set of vectors in a vector space $V$. Such a set is called convex if for all vectors $\mathbf{v}$ and $\mathbf{w}$ that are in $\mathscr{A}$, the set $\mathscr{A}$ contains the complete line segment connecting $\mathbf{v}$ and $\mathbf{w}$. The convex hull of $\mathscr{A}$, denoted by conv( $\mathscr{A})$, refers to the smallest convex set in $V$ that contains $\mathscr{A}$ (In other words, $\operatorname{conv}(\mathscr{A})$ is the intersection of all 
convex sets that contain $\mathscr{A})$. The set $\operatorname{conv}(\mathscr{A})$ may also be defined as the set of all convex combinations of finite sets of vectors taken from $\mathscr{A}$. If $\mathscr{A}$ is a convex set then $\operatorname{conv}(\mathscr{A})$ is identical to $\mathscr{A}$.

Orthogonality: If $\mathbf{X}=\left[X_{1}, X_{2}, \ldots, X_{N}\right]$ and $\mathbf{Y}=\left[Y_{1}, Y_{2}, \ldots, Y_{N}\right]$ are vectors in $\mathbb{R}^{N}$, their (standard) dot product (or scalar product) is defined by,

$$
\mathbf{X} \cdot \mathbf{Y}=X_{1} Y_{1}+X_{2} Y_{2}+\ldots \ldots+X_{N} Y_{N}
$$

Two vectors $\mathbf{X}$ and $\mathbf{Y}$ are orthogonal if $\mathbf{X} \cdot \mathbf{Y}=\mathbf{0}$. (Here $\mathbf{0}$ is the zero vector.)

\subsection{Stoichiometric constraints}

For a given chemical reaction network with kinetics, it is possible to ascertain constraints on product yield compositions based on stoichiometry.

Stoichiometric constraints serve as outer bounds for both the pure reactor synthesis problem and the reactor-separator synthesis problem described in Chapter 3.

The goal of this section is to informally describe how one can determine stoichiometric bounds on attainable composition space without rigorous proofs. Although the theory underlying calculation of stoichiometric bounds is not deep, the formulation presented here will be drawn upon when kinetic bounds are considered later in Chapters 5, 6 and 7. To simplify the discussion, we will limit the designs considered to isothermal steady-state designs involving only reactions and bulk mixing and in which the mixture density is constant and independent of composition. 
Consider [6] a reaction network as shown in eqn 4.1

$$
\begin{aligned}
& \mathrm{A}_{1} \rightleftharpoons \mathrm{A}_{2}+\mathrm{A}_{3} \\
& 2 \mathrm{~A}_{2} \rightarrow \mathrm{A}_{3} \rightleftharpoons \mathrm{A}_{4}+\mathrm{A}_{5}
\end{aligned} .
$$

This reaction network has five species and the production rate of each species is associated with a rate function, but this will not be relevant to this analysis. Our goal is to prescribe stoichiometric constraints based on a single feed stream for this system and also extend the idea to multiple feed streams as well.

We start by describing how one can create a reaction vector matrix for the given chemical reaction network. We denote a vector space of dimension $N$ by $\mathbb{R}^{N}$ where $N$ is the number of species in the reaction network. The standard basis for $\mathbb{R}^{N}$ is denoted by $\left\{\mathbf{e}_{1}, \mathbf{e}_{2}, \ldots, \mathbf{e}_{N}\right\}$, where,

$$
\mathbf{e}_{1}=[1,0, \ldots, 0], \mathbf{e}_{2}=[0,1, \ldots, 0], \ldots \ldots, \mathbf{e}_{N}=[0,0, \ldots, 1]
$$

For every reaction in the reaction network, we can write a reaction vector in $\mathbb{R}^{N}$ as shown in the following example: For the reaction $A_{1} \rightarrow A_{2}+A_{3}$, we can associate the reaction vector $\left(\mathbf{e}_{2}+\mathbf{e}_{3}\right)-\mathbf{e}_{1}=[-1,1,1,0,0]$ and for the reaction $A_{2}+A_{3} \rightarrow A_{1}$, we can associate the reaction vector $\mathbf{e}_{1}-\left(\mathbf{e}_{2}+\mathbf{e}_{3}\right)=[1,-1,-1,0,0]$. We obtain the full set of reaction vectors for the reaction in eqn (4.1) by continuing in this manner. 
The next step is to stack the reaction vectors to form a reaction vector

matrix as shown in eqn (4.2)

$$
\left[\begin{array}{rrrrr}
-1 & 1 & 1 & 0 & 0 \\
1 & -1 & -1 & 0 & 0 \\
0 & -2 & 1 & 0 & 0 \\
0 & 0 & -1 & 1 & 1 \\
0 & 0 & 1 & -1 & -1
\end{array}\right]
$$

The number of columns in the matrix equals the number of species in the reaction network and the number of rows equals the number of reactions in the system.

Each reaction vector also represent the net molecular gain of the various species due to the occurrence of that reaction. For example, the reaction $A_{3} \rightarrow A_{4}+A_{5}$, given by the reaction vector $[0,0,-1,1,1]$ indicates a loss of molecule $A_{3}$ and a gain of a molecule of $\mathrm{A}_{4}$ and $\mathrm{A}_{5}$ due to the occurrence of this reaction. The molecular populations of $A_{1}$ and $A_{2}$ are unaffected by the occurrence of this reaction.

The span of the reaction vectors is known as the stoichiometric subspace. (This is an important concept that will be used in later chapters of this thesis.) Stated more formally, the stoichiometric subspace is the linear subspace in $\mathbb{R}^{N}$ comprised of all the vectors that are linear combinations of the reaction vectors. The dimension of the stoichiometric subspace is equal to the number of members in the largest linearly independent set of reaction vectors. The dimension of the stoichiometric subspace can be ascertained by computing the rank of the reaction vector matrix using standard methods like row reduction $[83,90]$.

For the reaction network in eqn. (4.1), using the ideas mentioned, one can determine the dimension of the stoichiometric subspace to be equal to three (the rank of 
the reaction vector matrix is determined to be three.) A set of linearly independent reaction vectors is,

$$
\{[-1,1,1,0,0],[0,-2,1,0,0],[0,0,-1,1,1]\} \text {. }
$$

Eqn. (4.3) represents a set of vectors that form a basis for the stoichiometric subspace for this reaction network, and all vectors in the subspace will have the form

$$
\alpha[-1,1,1,0,0]+\beta[0,-2,1,0,0]+\gamma[0,0,-1,1,1]=[-\alpha, \alpha-2 \beta, \alpha+\beta-\gamma, \gamma, \gamma],
$$

where $\alpha, \beta$ and $\gamma$ can be any real number.

The stoichiometric subspace is of special importance for a chemical

reaction network because it contains all vectors in $\mathbb{R}^{N}$ that might represent compositional changes occurring due to the reactions. This means that if the composition changes from $\mathbf{c}^{\prime}$ to $\mathbf{c}^{\prime \prime}$ due to the occurrence of chemical reactions, then $\mathbf{c}^{\prime \prime}-\mathbf{c}^{\prime}$ will lie in the stoichiometric subspace. The composition change $\mathbf{c}^{\prime \prime}-\mathbf{c}^{\prime}$ will also conform to eqn (4.4) in the sense that one is able to find real values $\alpha, \beta$ and $\gamma$ that satisfy the equality.

\subsubsection{Compositions stoichiometrically compatible with the feed}

Consider a steady-state reactor configuration of arbitrary design with a single feed stream of composition $\mathbf{c}^{f}$ and a single output from the system of composition $\mathbf{c}$, then $\mathbf{c}^{f}-\mathbf{c}$ will reside in the stoichiometric subspace of the reaction network. If $\mathbf{S} \subset \mathbb{R}^{N}$ denotes the stoichiometric subspace, then one can say that $\mathbf{c}$ lies in the affine subspace $\mathbf{c}^{f}+\mathbf{S}$. 
For a system with a single feed stream $\mathbf{c}^{f}$ and known kinetics, the previous discussions suggest that all achievable compositions are constrained to lie in the affine subspace $\mathbf{c}^{f}+\mathbf{S}$. The constraints that arise due to this condition along with nonnegativity requirements for individual species concentrations serve as the stoichiometric bounds on feasible compositions for this system. For the reaction shown in equation (4.1), if there is a single feed stream such that $\mathbf{c}^{f}=[1,0,0,0,0]$ then any stoichiometrically feasible composition must also satisfy the following equality obtained by adding $\mathbf{c}^{f}$ to the right-hand-side of eqn (4.4)

$$
\mathbf{c}=[1-\alpha, \alpha-2 \beta, \alpha+\beta-\gamma, \gamma, \gamma]
$$

where $\alpha, \beta$ and $\gamma$ can be any real valued number. All compositions that conform to this equality are stoichiometrically compatible with the feed.

For a system with multiple feed streams, the computation is done a little differently, as explained below. The goal is to find stoichiometric constraints on feasible composition space for a given reaction system with $p$ separate feed streams of compositions $\mathbf{c}^{f_{1}}, \mathbf{c}^{f_{2}}, \ldots, \mathbf{c}^{f_{p}}$. By combining the feed streams in varying proportions, all compositions in the convex hull of the set of feed streams is also attainable. If $\mathbb{F}:=\left\{\mathbf{c}^{f_{1}}, \mathbf{c}^{f_{2}}, \ldots, \mathbf{c}^{f_{p}}\right\}$ then one can write the convex hull of this set as

$$
\operatorname{conv}(\mathbb{F}):=\left\{\sum_{i=1}^{p} \lambda_{i} \mathbf{c}^{f_{i}}: \sum_{i=1}^{p} \lambda_{i}=1,0 \leq \lambda_{i} \leq 1, i=1,2, \ldots p\right\} .
$$

Similar to the single feed example, one can assume that $\mathbf{c}^{\mathrm{in}}$ is the composition of a stream that serves as feed to a reactor of arbitrary design and $\mathbf{c}^{\text {out }}$ is the composition of the output stream. Since $\mathbf{c}^{\text {in }}$ is the composition of the stream that is obtained through mixing of the 
feed streams in varying proportions, it is a member of $\operatorname{conv}(\mathbb{F})$. From the earlier

discussion (for single feed streams) one can deduce that $\mathbf{c}^{\text {out }}$ should reside in the affine subspace $\mathbf{c}^{\text {in }}+\mathbf{S}$ and one can see that all attainable compositions must lie in the set given by,

$$
\operatorname{conv}(\mathbb{F})+\mathbf{S}:=\left\{\mathbf{c} \in \mathbb{R}^{\mathbf{N}}: \mathbf{c}=\mathbf{c}^{\prime}+\boldsymbol{\varepsilon}, \mathbf{c}^{\prime} \in \operatorname{conv}(\mathbb{F}), \boldsymbol{\varepsilon} \in \mathbf{S}\right\}
$$

As described in Feinberg [6], the set $\operatorname{conv}(\mathbb{F})+\mathbf{S}$ is not typically an affine subspace of $\mathbb{R}^{N}$ but it will be often true that the set is contained within an affine space of dimension less than $N$. Any vector in the set $\operatorname{conv}(\mathbb{F})$ can be represented by the form

$$
\begin{aligned}
& \mathbf{c}^{f_{1}}+\lambda_{2}\left(\mathbf{c}^{f_{2}}-\mathbf{c}^{f_{1}}\right)+\lambda_{3}\left(\mathbf{c}^{f_{3}}-\mathbf{c}^{f_{1}}\right)+\ldots . . \lambda_{k}\left(\mathbf{c}^{f_{p}}-\mathbf{c}^{f_{1}}\right), \\
& \sum_{i=2}^{k} \lambda_{i}=1,0 \leq \lambda_{i} \leq 1, i=2,3 \ldots k
\end{aligned}
$$

In other words, any vector in $\operatorname{conv}(\mathbb{F})$ can be represented by the form $\mathbf{c}^{f_{1}}+\boldsymbol{\delta}$ where $\boldsymbol{\delta}$ lies in the linear subspace $\Delta$ such that

$$
\Delta:=\operatorname{span}\left\{\mathbf{c}^{f_{2}}-\mathbf{c}^{f_{1}}, \mathbf{c}^{f_{3}}-\mathbf{c}^{f_{1}}, \ldots ., \mathbf{c}^{f_{p}}-\mathbf{c}^{f_{1}}\right\}
$$

Hence, any vector in $\operatorname{conv}(\mathbb{F})+\mathbf{S}$ can be represented by the form $\mathbf{c}^{f_{1}}+\boldsymbol{\delta}+\boldsymbol{\varepsilon}$. From this one can deduce that $\operatorname{conv}(\mathbb{F})+\mathbf{S}$ (and, hence, the feasible composition space) will lie in the affine subspace $\mathbf{c}^{f_{1}}+\Gamma \subset \mathbb{R}^{N}$, where

$$
\Gamma:=\Delta+\mathbf{S}
$$

This means that $\boldsymbol{\Gamma}$ is spanned by the reaction vectors along with the vectors that span $\Delta$. The dimension of $\boldsymbol{\Gamma}$ can be ascertained from the rank of the augmented matrix formed by the reaction vector matrix and the vectors spanning $\Delta$. This method of determining the stoichiometric constraints is demonstrated in the examples to follow. 
Assume a system with known kinetics as shown earlier in eqn (4.1) and assume that there are available two feed streams $\mathbf{c}^{f_{1}}=[1,0,0,0,0]$ and $\mathbf{c}^{f_{2}}=[0,1,0,0,0]$. One starts by computing $\mathbf{c}^{f_{2}}-\mathbf{c}^{f_{1}}:=[-1,1,0,0,0]$ and then augment this to the reaction vector matrix in eqn (4.2) to give

$$
\left[\begin{array}{rrrrr}
-1 & 1 & 1 & 0 & 0 \\
1 & -1 & -1 & 0 & 0 \\
0 & -2 & 1 & 0 & 0 \\
0 & 0 & -1 & 1 & 1 \\
0 & 0 & 1 & -1 & -1 \\
-1 & 1 & 0 & 0 & 0
\end{array}\right] .
$$

The rank of this matrix is computed to be four and the reaction stoichiometry will constrain compositions to lie in the four-dimensional affine space $\mathbf{c}^{f_{1}}+\boldsymbol{\Gamma}$, where $\boldsymbol{\Gamma}$ is the linear subspace in $\mathbb{R}^{5}$ spanned by the rows in the matrix in eqn (4.10).

A basis for this matrix is determined by the vectors,

$$
\{[-1,1,1,0,0],[0,-2,1,0,0],[0,0,-1,1,1],[-1,1,0,0,0]\}
$$

and any vector in $\Gamma$ will be given by linear combinations of these vectors:

$$
\begin{aligned}
& a[-1,1,1,0,0]+b[0,-2,1,0,0]+c[0,0,-1,1,1]+d[-1,1,0,0,0] \\
& =[-a-d, a-2 b+d, a+b-c, c, c]
\end{aligned}
$$

where $a, b, c$ and $d$ are any choice of real numbers. Similar to the computation done for single feed streams, all feasible compositions for this example must satisfy the following equality,

$$
\mathbf{c}=[1-a-d, a-2 b+d, a+b-c, c, c],
$$


where $a, b, c$ and $d$ are real numbers. The equation prescribed by eqn (4.11) along with the non-negativity condition of individual species concentrations will give the stoichiometric bounds that constrain the feasible composition space.

\subsubsection{Methods to compute stoichiometric constraints}

Here we describe two methods to compute stoichiometric constraints for a reaction network with a single feed stream. Take the reaction following reaction network described earlier:

$$
\begin{aligned}
& \mathrm{A}_{1} \rightleftharpoons \mathrm{A}_{2}+\mathrm{A}_{3} \\
& 2 \mathrm{~A}_{2} \rightarrow \mathrm{A}_{3} \rightleftharpoons \mathrm{A}_{4}+\mathrm{A}_{5}
\end{aligned}
$$

It was shown in that section that for a single feed stream of composition $\mathbf{c}^{f}=[1,0,0,0,0]$ the compositions stoichiometrically compatible with the feed will have the general form

$$
\mathbf{c}=[1-\alpha, \alpha-2 \beta, \alpha+\beta-\gamma, \gamma, \gamma]
$$

where $\alpha, \beta$ and $\gamma$ can be any real valued number. Making the assertion that each of the species concentrations be nonnegative will give the following constraints:

$$
1-\alpha \geq 0 ; \alpha-2 \beta \geq 0 ; \alpha+\beta-\gamma \geq 0 ; \gamma \geq 0
$$

which lead to the following constraints,

$$
0 \leq \alpha \leq 1 ; 0 \leq \beta \leq \frac{1}{2} ; 0 \leq \gamma \leq \frac{3}{2}
$$

Hence for different values of $\alpha, \beta$ and $\gamma$ that satisfy the above constraints, one can determine the entire set of compositions that are stoichiometrically compatible with the given feed composition. 
A second method is presented below that is computationally easier to implement. If $\mathbf{c}$ is any composition that is stoichiometrically compatible with the feed composition $\mathbf{c}^{f}$, the vector $\left(\mathbf{c}-\mathbf{c}^{f}\right)$ will reside in the stoichiometric subspace $\mathbf{S}$ corresponding to that reaction network. For the example given, we let $\mathbf{M}=\left[M_{1}, M_{2}, M_{3}, M_{4}, M_{5}\right]$ be any vector that is orthogonal to all vectors in $\mathbf{S}$, then we can write,

$$
\mathbf{M} \cdot\left(\mathbf{c}-\mathbf{c}^{f}\right)=\mathbf{0}
$$

In general, for a reaction network with $N$ species, we can say that all vectors that lie in $\mathbb{R}^{N}$ but are orthogonal to every vector in $\mathbf{S}$ lie in the linear subspace, $\mathbf{S}^{\perp}$. We can denote a basis for $\mathbf{S}^{\perp}$ by the set of vectors:

$$
\left\{\mathbf{M}_{1}, \mathbf{M}_{2}, \ldots . ., \mathbf{M}_{p}\right\}
$$

If $s$ is the dimension of the stoichiometric subspace then it can be shown that [83] $p=N-s$. For every $\mathbf{M}_{\theta}$ that constitutes a basis for $\mathbf{S}^{\perp}$, we can rewrite equation (4.12) as ,

$$
\mathbf{M}_{\theta} \cdot\left(\mathbf{c}-\mathbf{c}^{f}\right)=\mathbf{0}
$$

A basis for $\mathbf{S}^{\perp}$ in the above example can be computed in the usual manner [90] and in this case, it turns out to be,

$$
\{[3,1,2,2,0],[0,0,0,-1,1]\} \text {. }
$$

Assuming that $\mathbf{c}=\left[c_{1}, c_{2}, c_{3}, c_{4}, c_{5}\right]$ we apply equation (4.13) for each of vectors in (4.14) to get the following equations, 


$$
\begin{aligned}
& 3 c_{1}+c_{2}+2 c_{3}+2 c_{4}=3 \\
& -c_{4}+c_{5}=0
\end{aligned}
$$

Using equation (4.15) and the non-negativity constraint for each of the species

concentrations, we can write the following constraints representing the set of $c_{1}, c_{2}, c_{3}$

values that are stoichiometrically compatible with the feed.

$$
\begin{aligned}
& c_{1} \geq 0 \\
& c_{2} \geq 0 \\
& c_{3} \geq 0 \\
& 3 c_{1}+c_{2}+2 c_{3} \leq 3
\end{aligned}
$$

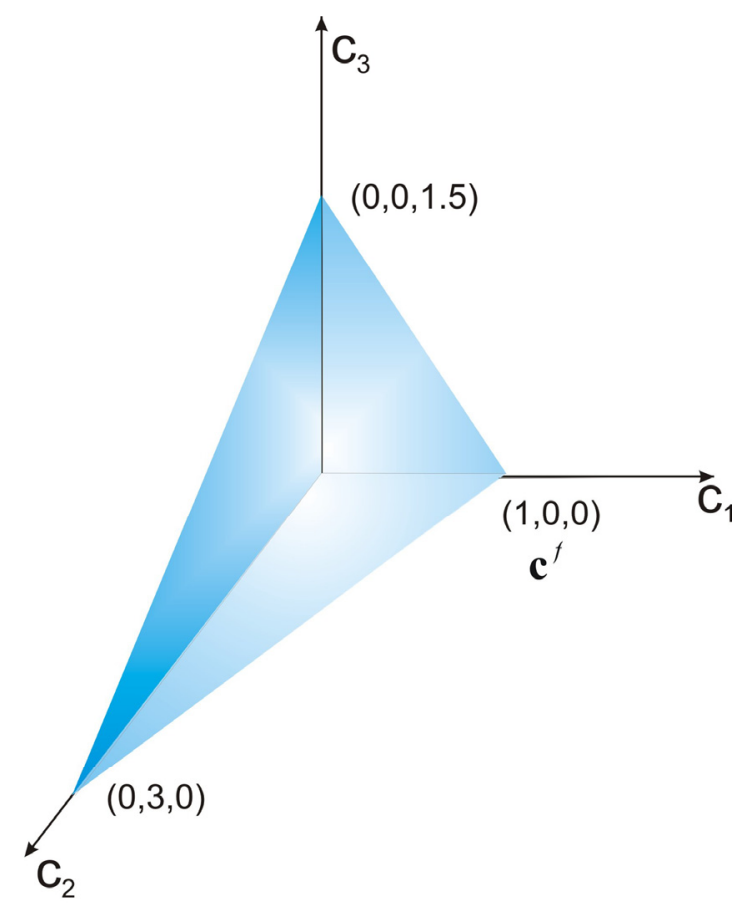

Figure 4.1 A tetrahedron in $\mathbb{R}^{3}$ of compositions stoichiometrically compatible with the feed 
The set of $c_{1}, c_{2}, c_{3}$ values that are stoichiometrically compatible with the feed are shown pictorially in Figure 4.1 and consist of all the values residing in the tetrahedron with vertices $[1,0,0],[0,3,0],[0,0,1.5]$ and $[0,0,0]$.

Note that though there are five species, the stoichiometric constraints are described in three dimensions. However, the concentrations $c_{4}$ and $c_{5}$ cannot have any arbitrary value but only those that satisfy the inequalities in (4.16) as well as the equations in (4.15).

A full representation of the stoichiometric constraints on all the concentrations can be written as follows:

$$
\begin{aligned}
& c_{1} \geq 0 \\
& c_{2} \geq 0 \\
& c_{3} \geq 0 \\
& 3 c_{1}+c_{2}+2 c_{3} \leq 3 \\
& c_{4}=\frac{1}{2}\left(3-\left(3 c_{1}+c_{2}+2 c_{3}\right)\right) \\
& c_{5}=c_{4} .
\end{aligned}
$$

Equation (4.17) shows how every composition in $\mathbb{R}^{5}$ that is stoichiometrically compatible with the given feed is constrained.

\subsection{Thermodynamic bounds}

One can use thermodynamic concepts to deduce bounds on feasible composition space but this section will be limited to a discussion of the basic ideas behind this computation and a discussion of why thermodynamic bounds might not be useful in some instances, even relative to stoichiometric bounds. 
Given a reaction network with known kinetics, one is interested in describing limits imposed on feasible composition space (and hence bounds on the attainable region for the pure reactor synthesis problem) by thermodynamic considerations. For a closed system, it can be shown that the change in free energy associated with the system is always less than or equal to zero. In other words, the free energy either remains the same or decreases over time. This concept is applied to a system that permits reaction and mixing alone as described in the simple example below.

Consider a simple reaction with two species,

$$
\mathrm{A}_{1} \underset{0.5}{\stackrel{1}{\rightleftharpoons}} \mathrm{A}_{2}
$$

having rate constants as shown and assume that the kinetics is mass-action. If our twospecies mixture were an ideal gas, then its Helmholtz free energy function would have the form[83]:

$$
\mathrm{h}(\mathbf{c}):=\sum_{i=1}^{2}\left[c_{i}\left(\ln c_{i}-\ln c_{i}^{*}-1\right)+c_{i}^{*}\right]
$$

where $c_{i}$ is the concentration (in moles/volume) of species $\mathrm{A}_{\mathrm{i}}$ and $\left[c_{1}^{*}, c_{2}^{*}\right]$ is an equilibrium composition.

We look at two different feed stream compositions fed to the system and make conclusions about what thermodynamics tells us about the feasible composition space. Firstly, assume a feed stream to the system of composition $\mathbf{c}^{f}=[1,0]$. We then compute the equilibrium compositions based on this composition and the given kinetics (derived from eqn 4.18). Using eqn (4.19) we compute $\mathrm{h}\left(\mathbf{c}^{f}\right)$ to ascertain the free energy associated with the feed stream composition (in this case, the free energy function was 
evaluated close to the feed by using a limit function). Since the free energy within the system has to either decrease or remain the same, one can use eqn (4.19) to find the surface (or curve) in composition space where the free energy remains the same and this will form the thermodynamics-based outer bounds. If the free energy of the system decreases, all such compositions must lie inside this curve.

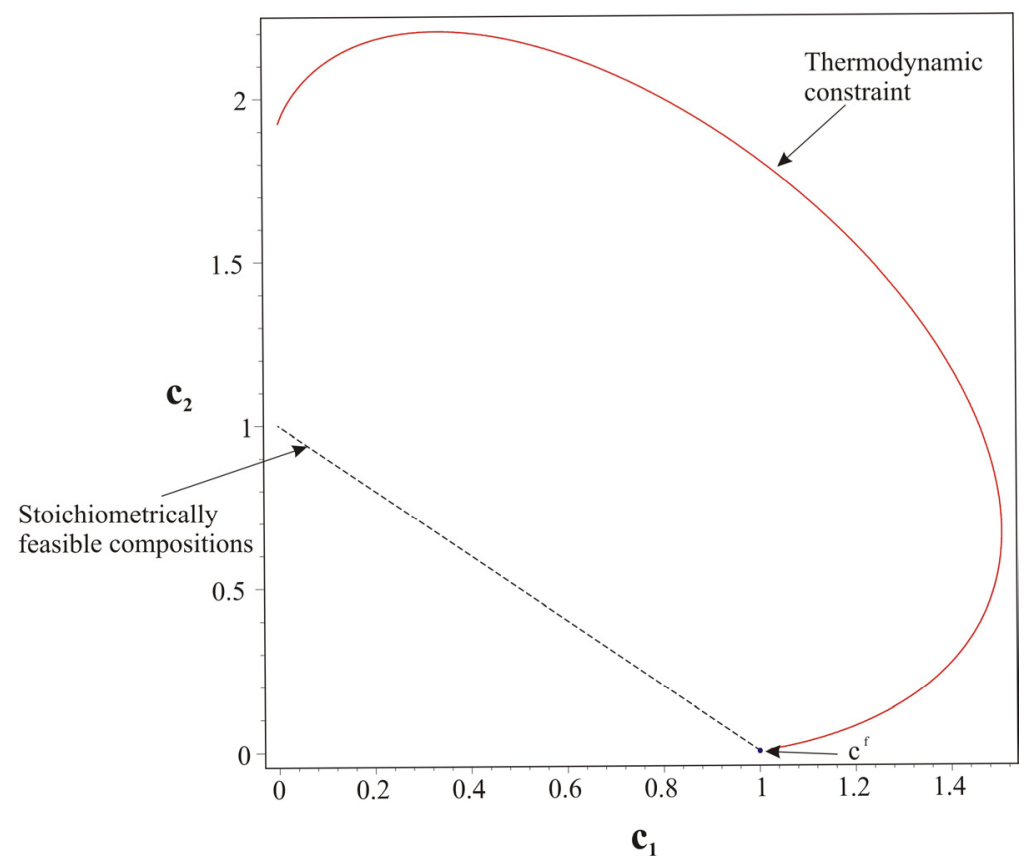

Figure 4.2 Comparing thermodynamic constraints and stoichiometrically feasible compositions for a feed stream composition $[1,0]$

Figure 4.2 shows a solid curve that represents the thermodynamic constraint calculated as explained and the dotted line represent the stoichiometrically feasible compositions computed by the methods explained in Section 4.2 (Any composition on the dotted line is stoichiometrically compatible with the feed composition given). It is clear that the thermodynamic constraint does not provide any new or useful information in terms of feasible composition space. 
The computation is repeated for a feed stream composition of $\mathbf{c}^{f}=[0.5,0.5]$ and the results are displayed in Figure 4.3. The solid curve represents the thermodynamic constraint while the dotted line represents the stoichiometrically feasible compositions.

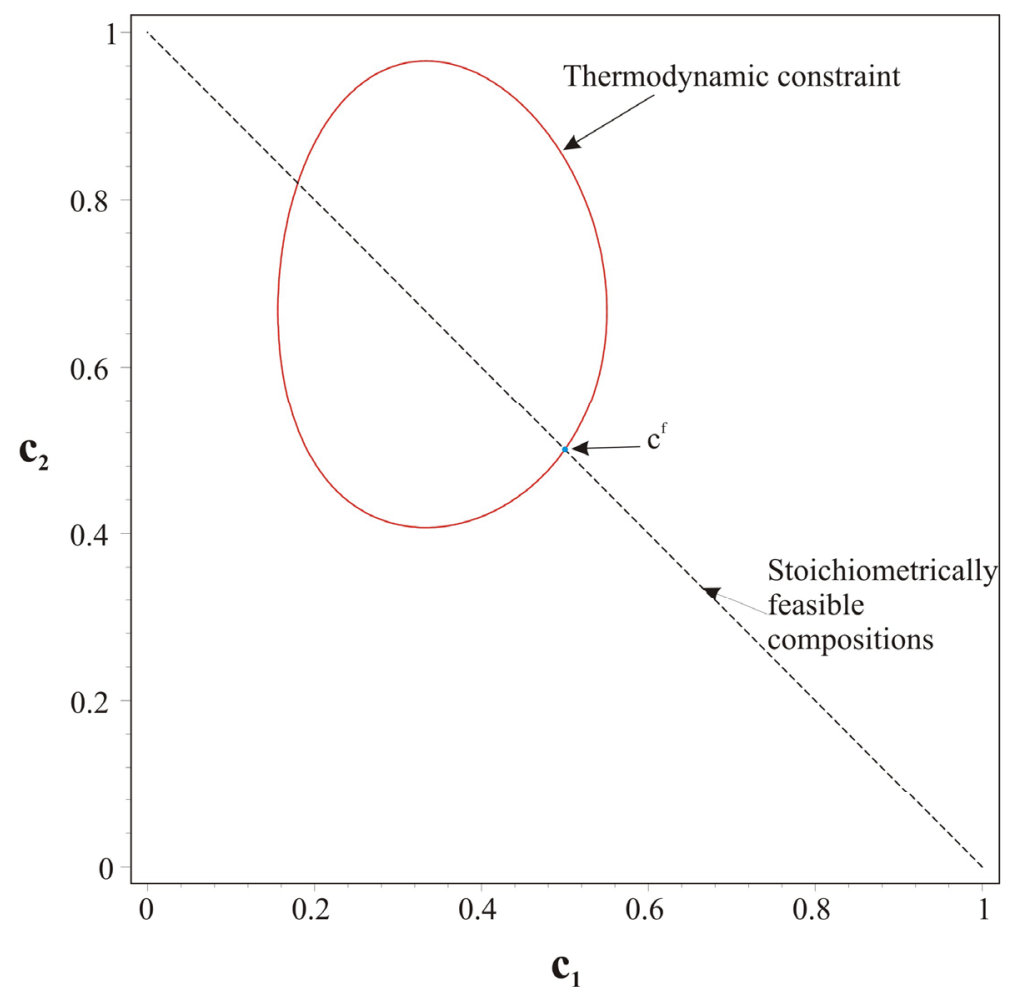

Figure 4.3 Comparing thermodynamic constraints and stoichiometrically feasible compositions for a feed stream composition of $[0.5,0.5]$

From the first computation, Figure 4.2 shows that the thermodynamic constraint is really broad and does not give much useful information. In fact for a feed stream of composition $[1,0]$, the stoichiometric constraints serve better in revealing 
information about feasible compositions. In the second computation, the thermodynamic constraint for a feed stream of composition $[0.5,0.5]$ does give some useful information and some that is not so valuable. While it serves to preclude a lot of the stoichiometrically feasible space it also encompasses some composition space that is not stoichiometrically feasible. From Figure 4.3 one might then conclude that the feasible composition space resides in a region suggested as feasible by both the constraints.

The two examples described above are meant to demonstrate how thermodynamic constraints might not be reliable in bounding the attainable region for the pure reactor synthesis problem. The computation might or might not be successful in finding meaningful constraints on feasible composition space; and conditions for when the thermodynamic constraints do give useful information is not fully understood.

An article by Shinnar \& Feng [84] describes a method using ideas similar to those mentioned in this section. The authors describe how one can use thermodynamic concepts to analyze complex reaction systems with several reactions like those encountered in the study of complex catalytic reactions. The bounds derived by the authors are based on the following concept: every "independent" chemical reaction must proceed in the direction of decreasing free energy. The bounds are generated without applying the specific kinetics of the system but rather are based on the structure of the individual reactions. The methods discussed in the article are more suited in studying reactions over a wide range of operating conditions and different catalysts. For reactions with specific kinetics this method may or may not provide useful information as seen in the examples worked out in this section. 


\subsection{Reactor-separator bounds}

As discussed in Chapter 3, there is no known method by which one can construct the full attainable region for the pure reactor synthesis problem with certainty. However, certain results do describe how conventional reactors might shape the boundary of this attainable region. The aim of this thesis is to prescribe outer bounds on the pure reactor attainable region. One approach that we considered was the use of ideas from the reactor-separator synthesis problem as described in this section.

The pure reactor and the reactor-separator problem are two opposing extreme designs in process synthesis. Process designs that utilize separations most likely produce more of a desired chemical species than in the absence of separations. If the true pure reactor attainable region (AR) were known, it would always be bounded on the outside by the reactor-separator AR. A comparison of the reactor-separator AR and a pure reactor synthesis candidate attainable region, will enable us to determine how close a candidate may be to the actual pure reactor AR. In addition, these are true bounds (though not the best) on the pure reactor AR. We begin by reviewing the reactorseparator synthesis problem followed by an example showing how the approach was used to determine outer bounds on the reactor synthesis problem.

The reactor-separator synthesis problem seeks to resolve [81], for a given chemistry, the limits on what might be attained from a given feed allowing all possible steady-state reactor-separator designs. More detailed constraints are also imposed on this problem in the form of pressure limitations, temperature limitations, unwanted sideproduct constraints etc. 
In this problem, rather than investigate those specific designs that help one achieve the maximum molar effluent rate of the desired species (subject to the aforementioned constraints), the focus has been on computing the value of the maximum molar effluent rate itself for it serves as a benchmark against which existing designs may be evaluated.

The resolution of this problem involves the use of the CFSTR Equivalence Principle as stated by Feinberg \& Ellison [81]. This principle states that for a given reaction kinetics, any molar effluent composition that can be attained by a reactor separator design can also be attained arbitrarily closely by another steady state reactorseparator design in which the reactor components consist of no more than $s^{+1}$ CFSTRs, where $s$ is the rank of the reaction network (see ref [82] for details). Also the reactor volume remains the same in the equivalent system. This principle thus serves to determine the attainable region of molar effluent rate vectors exactly and serves to determine the bounds for it.

Consider a species formation rate function $\mathbf{r}(\mathbf{c}, \mathrm{T})$, which is a function of both composition and temperature. Consider all steady-state reactor separator designs in which the $(\mathbf{c}, \mathrm{T})$ states within reactor components are constrained to lie in a closed and bounded set $\Omega \subset \mathbb{R}^{N+1}$, and in which the total reactor volume lies within the closed interval $\mathscr{V}:=[0, \overline{\mathrm{V}}]$

Using the equivalence principle it is possible to describe the attainable region as follows: If the set $\boldsymbol{M}$ denotes the set of all attainable molar-effluent-rate vectors and $\mathbf{M}^{\mathbf{0}}$ represents the molar-feed-rate vector, then: 


$$
\mathcal{M}=\operatorname{conv}\left(\mathbf{M}^{0}+\mathcal{V} \cdot \mathbf{r}(\Omega)\right) \cap \mathbb{R}_{+}^{N}
$$

where conv(.) denotes taking the convex hull, $\mathbb{R}_{+}^{N}$ denotes the positive orthant of the $N$ dimensional vector space and

$$
\mathbf{M}^{\mathbf{0}}+\mathcal{V} \cdot \mathbf{r}(\Omega)=\left\{\mathbf{M} \in \mathbb{R}^{N}: \mathbf{M}=\mathbf{M}^{\mathbf{0}}+\mathrm{V} \cdot \mathbf{r}(\mathbf{c}, T), \mathrm{V} \in \mathcal{V},(\mathbf{c}, \mathrm{T}) \in \Omega\right\}
$$

In Feinberg and Ellison [81], the above principles were used in calculating bounds for the Van de Vusse example including the effects of temperature in rate constants and imposing constraints on pressure, temperature and maximum reactor volume. It is clear to see that the CFSTR equivalence principle is powerful in that, for the purpose of assessing what is attainable, it narrows an almost limitless variety of reactorseparator configurations down to a configuration that constitutes a small number of CFSTRs.

The reactor-separator problem is studied by finding attainable molar effluent rates rather than attainable compositions. We begin by first deriving a relationship between the two for a special case.

Assume now that we have a reactor-separator system with $N$ chemical species and mixtures of constant temperature and density alone. Let the molar feed rate vector to the system be denoted by $\mathbf{M}^{0}:=\left[M_{1}^{0}, M_{2}^{0}, \ldots . ., M_{N}^{0}\right]$ where $M_{i}^{0}$ denotes the rate in moles/time of species $A_{i}$ fed into the system. Let the molar effluent rate vector of the system be denoted by $\mathbf{M}:=\left[M_{1}, M_{2}, \ldots . ., M_{N}\right]$ where $M_{i}$ denotes the rate in moles/time of species $A_{i}$ leaving the system. Let $g^{\text {in }}$ represent the total volumetric flow rate of streams entering the system (in units of volume/time). Let $g^{\text {out }}$ represent the total 
volumetric flow rate of streams leaving the system (in units of volume/time). Under steady state conditions, $g^{\text {in }}=g^{\text {out }}=g$. If we have a single feed stream of composition $\mathbf{c}^{f}$, then

$$
\mathbf{M}^{0}=g \mathbf{c}^{f} .
$$

Let the composition of the effluent stream be $\mathbf{c}$, then we can write:

$$
\mathbf{M}=g \mathbf{c} .
$$

Using equations (4.23) and (4.24) we can convert between molar flow rates and compositions for a fixed volumetric flow rate (subject to the assumed conditions of incompressibility and uniform density).

Our approach to using the reactor-separator methods described earlier can be elaborated as follows. We assume that $g=1$ (mol/unit vol) for equations (4.23) and (4.24) and that makes conversion between compositions and molar rates straightforward. The reactor synthesis problem allows for reactor volumes to be infinitely large whereas the reactor-separator problem restricts the total reactor volume in the system to a fixed value. To obtain meaningful bounds, we allow the total reactor volume for the latter problem to get extremely large. For the large volume we compute the full set of attainable molar effluent rates (i.e. we compute the full set $\mathbf{M}$ ) and this set will be the same as the full set of attainable compositions (by assuming $g=1$ in equation (4.24)) .

Figure 4.4 shows the results obtained for the irreversible Van de Vusse reaction network with the standard rate constants (as shown in equation 1.1). We assume a feed stream composition of $\mathbf{c}^{f}=[1,0,0,0]$ and assume $g=1$. Using equation (4.23) we get $\mathbf{M}^{0}=[1,0,0,0]$. By invoking equation (4.21) (for isothermal conditions) we can 

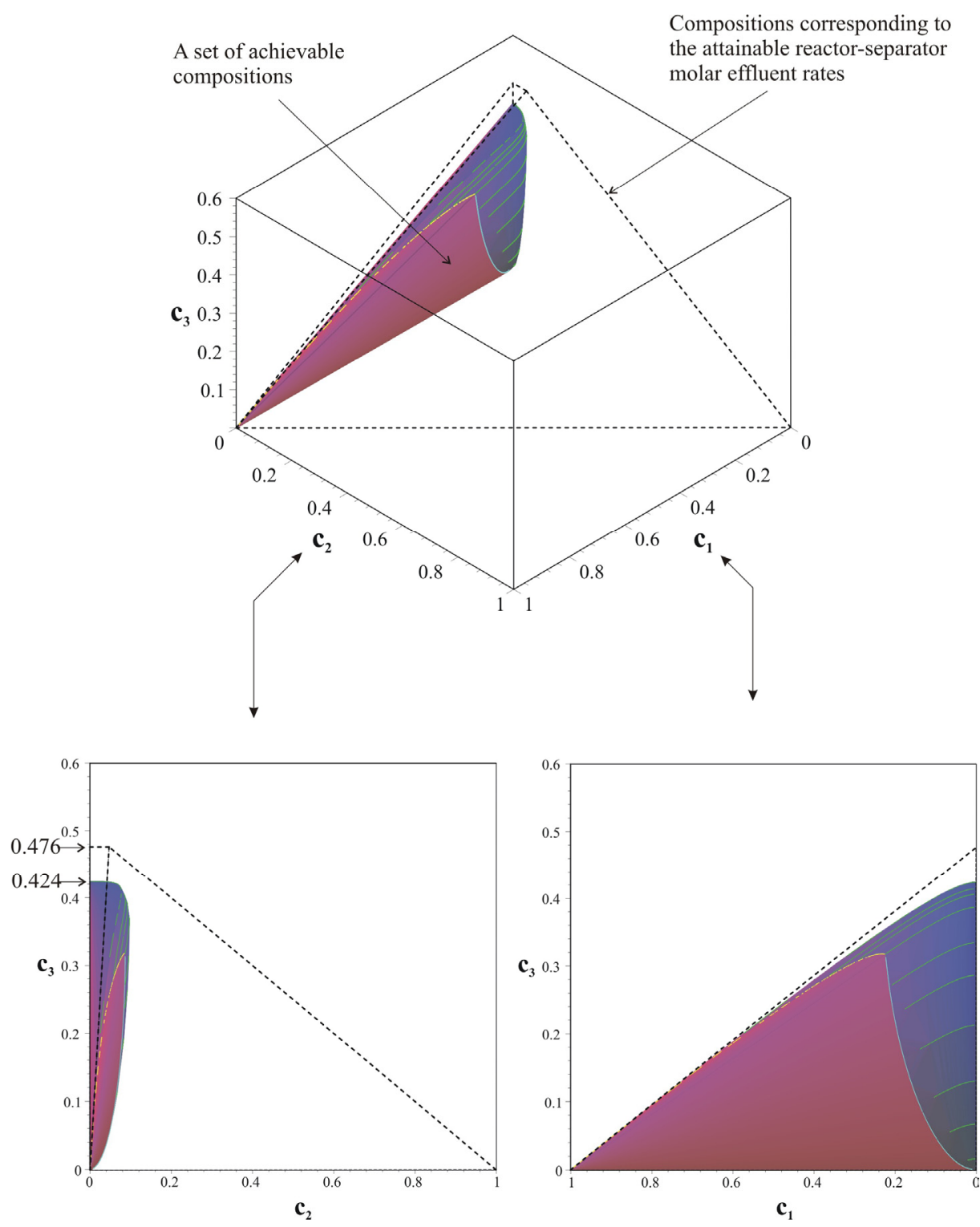

Figure 4.4 Comparison of a set of achievable compositions with compositions corresponding to the full set of attainable reactor-separator molar effluent rates

compute the full range of molar effluent rates for the system. We let $\Omega$ be equal to the set of compositions that are stoichiometrically compatible with the feed. The full set of effluent molar rates is computed and is converted to the corresponding set of 
compositions using equation (4.24). The boundary of the resulting set is plotted with dotted lines in Figure 4.4.

In Figure 4.4, the reactor-separator bounds are compared with a set of achievable compositions computed in the manner similar to Figure 1.1 in Chapter 1. The bounds do successfully bound the set of achievable compositions but they do not give much more information than the stoichiometric bounds for this system. The reactorseparator bounds show a maximum possible value of $c_{3}$ to be 0.476 while the stoichiometric limit is 0.5 (the largest realizable concentration of $c_{3}$ is found to be 0.424 ).

On one hand, it is extremely beneficial that we are able to compute exact and precise outer bounds on the compositions feasible through reactions alone by using results from the reactor-separator synthesis problem. The example worked out above shows that the results may be too broad or coarse to provide much useful information to the designer. The method of bounding hyperplanes to be described later in this thesis will prove to be more effective in precluding infeasible compositions than the methods described in this chapter. 


\section{CHAPTER 5}

\section{THE METHOD OF BOUNDING HYPERPLANES}

In this chapter, the goal is to describe in detail a novel method developed to construct outer bounds on the pure reactor attainable region. The first section of the chapter will be devoted to terminology and some preliminary ideas. In section 5.2, I describe how the method of bounding hyperplanes works, and in the final section more rigorous proofs are presented for the method presented. Material presented in this chapter is also presented in an article by Abraham and Feinberg [85].

\subsection{Preliminary ideas, terminology and notation}

To study process designs in composition space, one typically represents the compositions that might appear in a system with $N$ chemical species in a vector space of $N$-tuples of real numbers denoted by $\mathbb{R}^{N}$. The set of vectors in $\mathbb{R}^{N}$ with all components strictly nonnegative is denoted by $\mathbb{R}_{+}^{N}$. The dot product in $\mathbb{R}^{N}$ is typically computed as follows:

$$
\mathbf{x} \cdot \mathbf{y}=\left[x_{1}, x_{2}, \ldots . . x_{N}\right] \cdot\left[y_{1}, y_{2}, \ldots . y_{N}\right]:=x_{1} y_{1}+x_{2} y_{2}+\ldots+x_{N} y_{N}
$$


If $\mathbf{n}$ is a nonzero vector in $\mathbb{R}^{N}$ and $\gamma$ is a number, then one can define a hyperplane $H^{=}(\mathbf{n}, \gamma)$ as the set of vectors denoted by

$$
H^{=}(\mathbf{n}, \gamma):=\left\{\mathbf{x} \in \mathbb{R}^{N}: \mathbf{n} \cdot \mathbf{x}=\gamma\right\}
$$

A hyperplane generally divides a vector space into halfspaces. One can define halfspaces created by the hyperplane, $H^{=}(\mathbf{n}, \gamma)$, in $\mathbb{R}^{N}$ as follows. The two closed halfspaces, $H^{\leq}(\mathbf{n}, \gamma)$ and $H^{2}(\mathbf{n}, \gamma)$, can be defined as:

$$
\begin{aligned}
& H^{\leq}(\mathbf{n}, \gamma):=\left\{\mathbf{x} \in \mathrm{R}^{\mathrm{N}}: \mathbf{n} \cdot \mathbf{x} \leq \gamma\right\} \\
& H^{\geq}(\mathbf{n}, \gamma):=\left\{\mathbf{x} \in \mathrm{R}^{\mathrm{N}}: \mathbf{n} \cdot \mathbf{x} \geq \gamma\right\}
\end{aligned}
$$

In the same manner, one can define the open halfspaces, $H^{<}(\mathbf{n}, \gamma)$ and $H^{>}(\mathbf{n}, \gamma)$, such that

$$
\begin{aligned}
H^{<}(\mathbf{n}, \gamma) & :=\left\{\mathbf{x} \in \mathrm{R}^{\mathrm{N}}: \mathbf{n} \cdot \mathbf{x}<\gamma\right\} \\
H^{>}(\mathbf{n}, \gamma) & :=\left\{\mathbf{x} \in \mathrm{R}^{\mathrm{N}}: \mathbf{n} \cdot \mathbf{x}>\gamma\right\}
\end{aligned}
$$

A vector $\mathbf{x}^{*}$ in $\mathbb{R}^{N}$ is said to be a convex combination of vectors $\left\{\mathbf{x}^{\mathbf{1}}, \mathbf{x}^{2}, \ldots \mathbf{x}^{\mathbf{k}}\right\}$ also in $\mathbb{R}^{N}$ if there exist numbers $\left\{\lambda_{1}, \lambda_{2}, \ldots . . \lambda_{k}\right\}$ such that the following are all satisfied :

$$
\begin{aligned}
& 0 \leq \lambda_{j} \leq 1, j=1,2, \ldots ., k, \\
& \sum_{j=1}^{k} \lambda_{j}=1, \\
& \mathbf{x}^{*}=\sum_{j=1}^{k} \lambda_{j} \mathbf{x}^{j} .
\end{aligned}
$$


A set of vectors in $\mathbb{R}^{N}$ is said to be convex if every convex combination of members of the set is also a member of the set itself. Hence the closed half-spaces, open half-spaces, and the hyperplane defined earlier are all convex sets.

A hyperplane $H^{=}(\mathbf{n}, \gamma)$ is said to support [6] a set $\mathscr{C} \subset \mathbb{R}^{N}$ at a point $\mathbf{x}^{*} \in \mathscr{C}$ if $\mathbf{n} \cdot \mathbf{x}^{*}=\gamma$ and either

$\mathbf{n} \cdot \mathbf{x} \leq \gamma$ for all $\mathbf{x} \in \mathscr{C}$

or

$\mathbf{n} \cdot \mathbf{x} \geq \gamma$ for all $\mathbf{x} \in \mathscr{C}$.

That is, the hyperplane $H^{*}(\mathbf{n}, \gamma)$ supports $\mathscr{C}$ at $\mathbf{x}^{*}$ if $\mathbf{x}^{*}$ lies in the hyperplane and, moreover, the set $\mathscr{C}$ lies entirely in one of the closed half spaces defined by the hyperplane. In this case the hyperplane is called a support hyperplane.

The representation, $H^{=}(\mathbf{n}, \gamma)$ and $H^{=}(-\mathbf{n},-\gamma)$, both indicate the same vectors in $\mathbb{R}^{N}$ and are therefore the same hyperplane. To avoid ambiguity, throughout this thesis we follow the convention that when we use the representation $H^{=}(\mathbf{n}, \gamma)$ to indicate a support hyperplane for the set $\mathscr{C}$ at the point $\mathbf{x}^{*}$, then $\mathbf{n}$ and $\gamma$ are chosen such that

$\mathbf{n} \cdot \mathbf{x}^{*}=\gamma$ and

$\mathbf{n} \cdot \mathbf{x} \geq \gamma$ for all $\mathbf{x} \in \mathscr{C}$.

In particular, this rule requires that $\mathbf{n} \cdot\left(\mathbf{x}-\mathbf{x}^{*}\right) \geq \mathbf{0}$ for all $\mathbf{x} \in \mathscr{C}$. 
In our discussions it is presumed that the mixtures have a common fixed density independent of composition. This is a good approximation in the case of liquid mixtures in which the reactive solute species all have small concentrations relative to that of a solvent. The local composition of a mixture having $N$ species, $\mathrm{A}_{1}, \mathrm{~A}_{2}, \ldots . . \mathrm{A}_{N}$, can be described by a composition vector in $\mathbb{R}_{+}^{N}$ of the form $\mathbf{c}=\left[c_{1}, c_{2}, \ldots \ldots, c_{N}\right]$ where $c_{j}$ is the local molar concentration (in units of moles per unit volume) of species $\mathrm{A}_{j}$.

If a system has $k$ distinct streams having mixture compositions $\mathbf{c}^{1}, \mathbf{c}^{2} \ldots ., \mathbf{c}^{k}$ and volumetric flow rates $F_{1}, F_{2}, \ldots ., F_{k}$ respectively, then mixing these will give a new mixture composition. If the total volumetric flowrate is $F$, i.e. if

$$
F=F_{1}+F_{2}+\ldots .+F_{k},
$$

then, bulk mixing of these feed streams (in the absence of chemical reactions) will give a new stream having a composition $\mathbf{c}^{*}$ such that

$$
\mathbf{c}^{*}=\frac{1}{F} \sum_{j=1}^{k} F_{j} \mathbf{c}^{j}
$$

In other words, $\mathbf{c}^{*}$ will be a convex combination of mixture compositions $\mathbf{c}^{1}, \mathbf{c}^{2} \ldots ., \mathbf{c}^{k}$.

The species-formation-rate function, denoted by $\mathbf{r}(\cdot)$, derives from the kinetics for a given reaction network. If the local composition somewhere in the mixture is represented by the composition vector $\mathbf{c}$ then one can write the vector $\mathbf{r}(\mathbf{c})=\left[\mathrm{r}_{1}(\mathbf{c}), \mathrm{r}_{2}(\mathbf{c}), \ldots . ., \mathrm{r}_{N}(\mathbf{c})\right]$, where $\mathrm{r}_{j}(\mathbf{c})$ is the rate per unit volume at which species $\mathrm{A}_{j}$ is being produced in the mixture due to the occurrence of all chemical reactions. 
For a given species-formation-rate function, $\mathbf{r}(\cdot)$, there might exist a composition vector $\mathbf{c}^{*}$ such that $\mathbf{c}^{*} \in \mathbb{R}_{+}^{N}$ and $\mathbf{r}\left(\mathbf{c}^{*}\right)=\mathbf{0}$. If this is true, then $\mathbf{c}^{*}$ is an equilibrium composition of the function $\mathbf{r}(\cdot)$. If $\mathbf{r}\left(\mathbf{c}^{*}\right) \neq \mathbf{0}$ then $\mathbf{c}^{*}$ is termed as a nonequilibrium composition.

\subsection{Introduction to the method of bounding hyperplanes}

In this section, I will introduce the method of bounding hyperplanes using some of the preliminary ideas already described.

Consider an isothermal incompressible mixture of $N$ species denoted by $\mathrm{A}_{1}, \mathrm{~A}_{2}, \ldots \mathrm{A}_{N}$, and assume that there is available a single feed stream of composition $\mathbf{c}^{f} \in \mathbb{R}_{+}^{N}$. Moreover, let $\mathbf{r}(\cdot)$ be the species-formation-rate function for the mixture. The aim here is to describe outer bounds for the composition set in $\mathbb{R}_{+}^{N}$ that contain all the composition vectors that might be produced from $\mathbf{c}^{f}$ through any configuration of steadystate process designs that utilize reactions and mixing alone.

To begin the computation, one needs to start with a subset of $\mathbb{R}_{+}^{N}$ within which the desired compositions can be assumed to lie. This initial set can be large, and its bounds can be obtained by applying ideas of stoichiometric compatibility described in Chapter 4. For this discussion, assume that $\boldsymbol{C}^{0}$ is the set of all compositions that are stoichiometrically compatible with the feed stream composition $\mathbf{c}^{f}$. Figure 5.1 depicts such a set in a two-dimensional setting as a pentagon. The discussion of the method will 
evolve in two dimensions for the sake of clarity, but the techniques mentioned are not restricted to small dimensions. It will also typically be the case that all compositions that are stoichiometrically compatible with a given feed stream composition will be a polyhedron in $\mathbb{R}_{+}^{N}$.

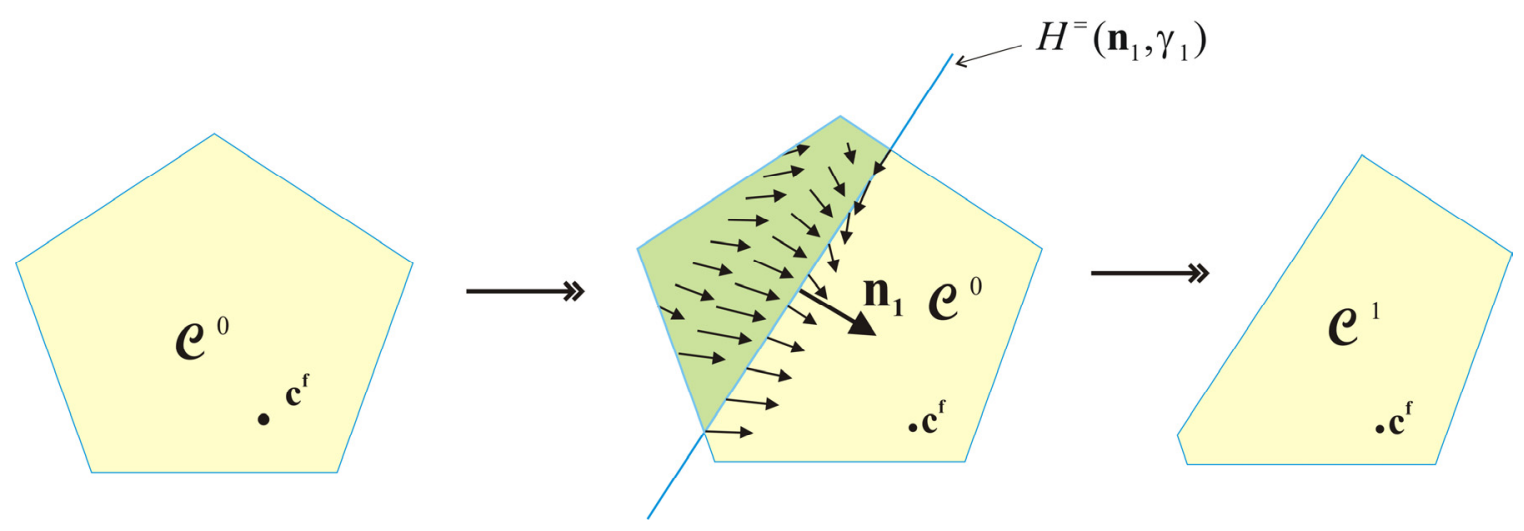

Figure 5.1 A single bound-trimming sequence on a composition set

The next step involves finding a hyperplane $H^{=}\left(\mathbf{n}_{1}, \gamma_{1}\right)$ (which is defined as in eqn 5.2) such that the following two conditions are satisfied: (1) the feed stream composition $\mathbf{c}^{f}$ lies in the half-space $H^{2}\left(\mathbf{n}_{1}, \gamma_{1}\right)$ and (2) all nonequilibrium compositions, $\mathbf{c} \in\left(\boldsymbol{C}^{0} \cap H^{\leq}\left(\mathbf{n}_{1}, \gamma_{1}\right)\right)$ satisfy the condition $\mathbf{n}_{1} \cdot \mathbf{r}(\mathbf{c})>0$. In two dimensions, a hyperplane is a straight line as depicted in Figure 5.1. The two mathematical conditions stated above can be restated in terms of the figure as follows: the line drawn (or the hyperplane $H^{=}\left(\mathbf{n}_{1}, \gamma_{1}\right)$ ) is such that the feed stream composition $\mathbf{c}^{f}$ lies on one side of (or on) this line, while for every nonequilibrium composition in the set $\boldsymbol{C}^{0}$ 
on the other side of (or on) the line, the species-formation-rate vector points toward the side containing $\mathbf{c}^{f}$. Figure 5.1 depicts this line and also shows smaller arrows that represent the direction of the rate vectors computed for compositions in the dark-shaded region $\left(\boldsymbol{e}^{0} \cap H^{\leq}\left(\mathbf{n}_{1}, \gamma_{1}\right)\right.$. One can see how the small arrows satisfy the condition that $\mathbf{n}_{1} \cdot \mathbf{r}(\mathbf{c})>0$ by observing that the small arrows make an acute angle with $\mathbf{n}_{1}$ (the normal to the hyperplane $\left.H^{=}\left(\mathbf{n}_{1}, \gamma_{1}\right)\right)$ and point outward from $H^{\leq}\left(\mathbf{n}_{1}, \gamma_{1}\right)$.

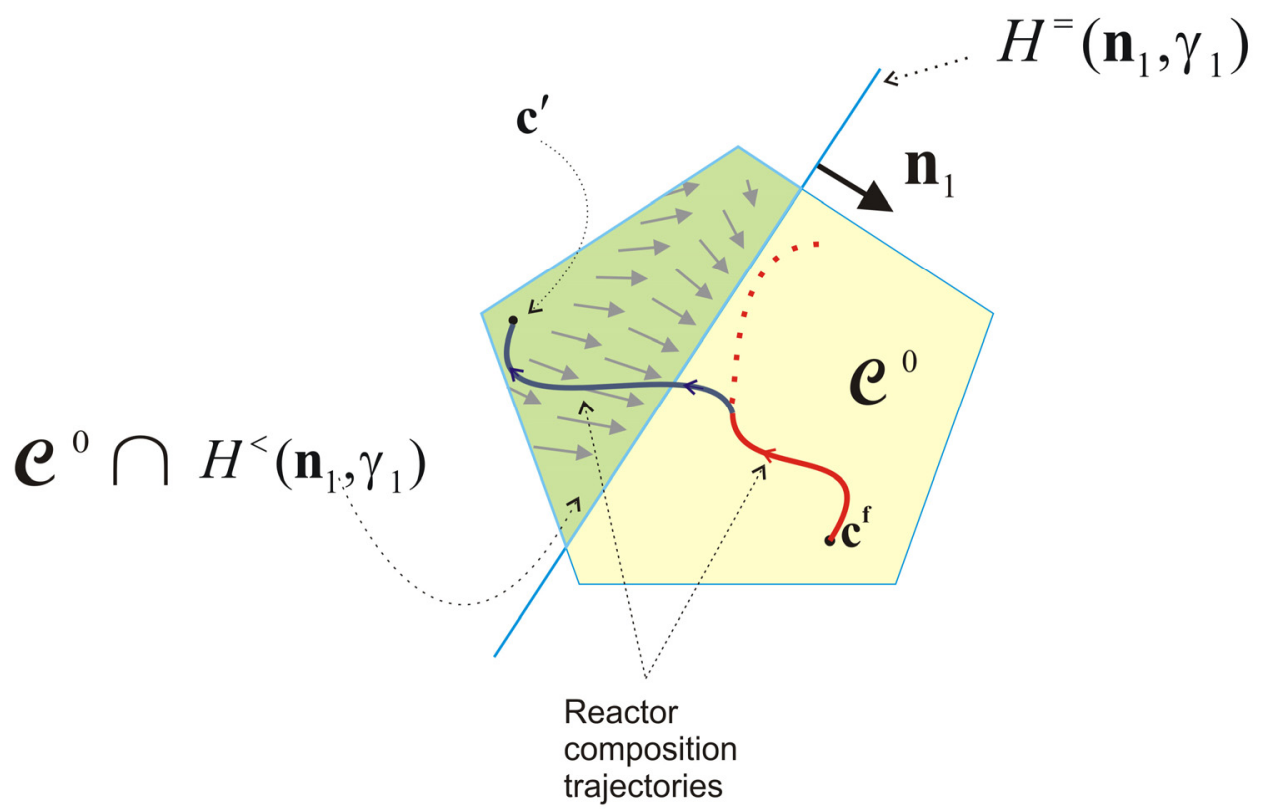

Figure 5.2 An impossible situation: No process design utilizing reaction and mixing alone with a feed stream $\mathbf{c}^{f}$ can reach the composition $\mathbf{c}^{\prime}$ in the manner shown

The claim to be made in this section is as follows: No steady-state design involving reactors and mixing alone and having a feed stream composition $\mathbf{c}^{f}$ can produce an effluent whose composition resides in the interior of the shaded region, 
$\boldsymbol{e}^{0} \cap H^{<}\left(\mathbf{n}_{1}, \gamma_{1}\right)$. The intuitive argument is that the outward-pointing reaction rate vectors in the shaded region would prevent trajectories to traverse into it from the feedcontaining side of the hyperplane $H^{=}\left(\mathbf{n}_{1}, \gamma_{1}\right)$. (A rigorous proof for this suggestion will be given in the following section.) If one accepts the argument that the shaded region is inaccessible from $\mathbf{c}^{f}$ then the region can be removed from $\boldsymbol{e}^{0}$ to give the tighter bounding region $\boldsymbol{e}^{1}$. Thus the hyperplane $H^{=}\left(\mathbf{n}_{1}, \gamma_{1}\right)$, while satisfying the two conditions stated earlier, also served to trim the initial bounding region $\boldsymbol{e}^{0}$ to give a smaller, more informative bounding region $\boldsymbol{C}^{1}$. The new set $\boldsymbol{e}^{1}$ will be referred to as the current polytope and will have the property that any composition that might be realized through steady state designs involving reactions and mixing alone with a feed stream of composition $\mathbf{c}^{f}$ must reside in that set.

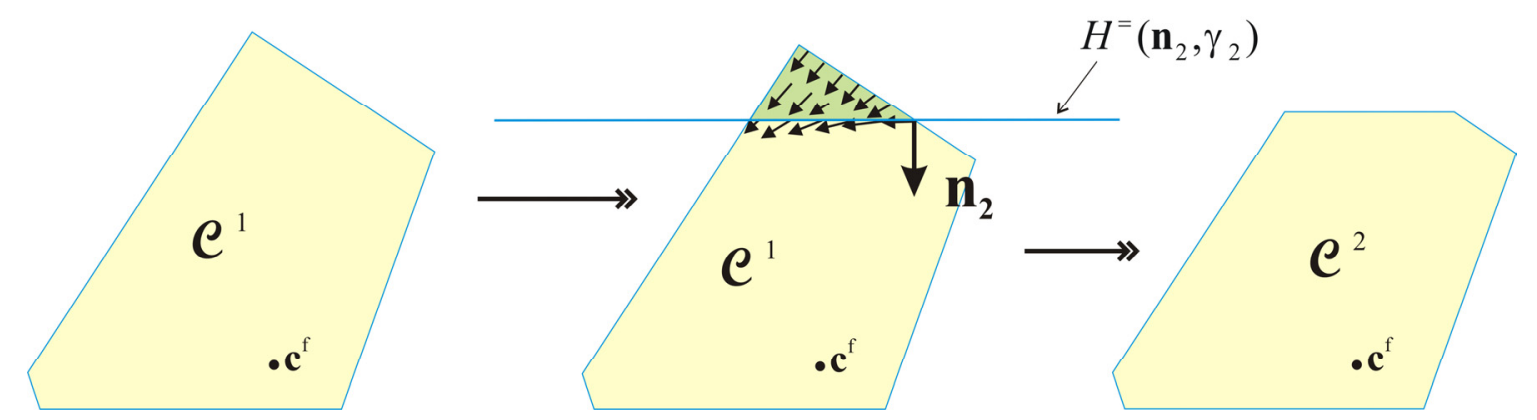

Figure 5.3 A second trimming sequence on a previously reduced composition set

One can repeat this procedure by starting with the set $\boldsymbol{e}^{1}$ and attempt to find a new hyperplane $H^{-}\left(\mathbf{n}_{2}, \gamma_{2}\right)$ such that the following two conditions are satisfied:

(1) the feed stream composition $\mathbf{c}^{f}$ lies in the half-space $H^{2}\left(\mathbf{n}_{2}, \gamma_{2}\right)$ and (2) all 
nonequilibrium compositions, $\mathbf{c} \in\left(\boldsymbol{C}^{1} \cap H^{\leq}\left(\mathbf{n}_{2}, \gamma_{2}\right)\right)$ satisfy the condition $\mathbf{n}_{2} \cdot \mathbf{r}(\mathbf{c})>0$. This is depicted in Figure 5.2 in the two dimensional setting where the hyperplane is a straight line and the small arrows represent the rate vectors computed for compositions in the shaded region. Again the argument can be made that no steady state design with reaction and mixing alone and having a feed stream composition $\mathbf{c}^{f}$ can achieve compositions in the shaded region due to the outward pointing vectors that impede attempts to access compositions in the shaded region. The argument then permits one to trim the current bounds on compositions attainable from $\mathbf{c}^{f}$ to produce the smaller bounding set $\boldsymbol{C}^{2}$ (a new current polytope) as shown in Figure 5.3.
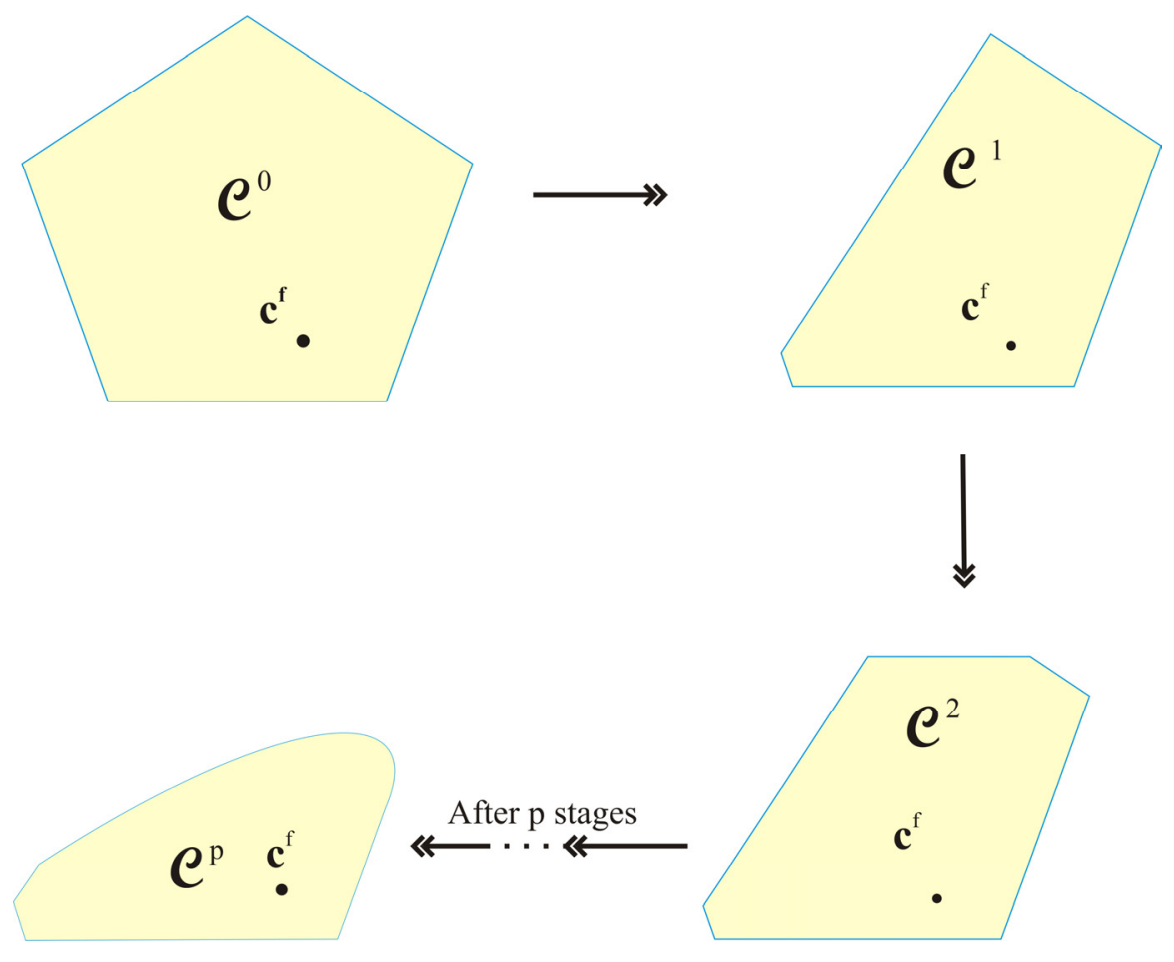

Figure 5.4 Complete bound trimming sequence for $p$ stages 
The bound trimming technique can be repeated for, say, $p$ total stages (see Figure 5.4) and during the $i^{t h}$ stage one can ask the following : Does there exist a hyperplane $H^{=}\left(\mathbf{n}_{i+1}, \gamma_{i+1}\right)$ corresponding to the current bounding set of compositions $\boldsymbol{e}^{i}$ (the current polytope) such that the following two conditions are satisfied : (1) the feed stream composition $\mathbf{c}^{f}$ lies in the half-space $H^{\geq}\left(\mathbf{n}_{i+1}, \gamma_{i+1}\right)$ and (2) all nonequilibrium compositions, $\mathbf{c} \in\left(\boldsymbol{e}^{i} \cap H^{\leq}\left(\mathbf{n}_{i+1}, \gamma_{i+1}\right)\right)$ satisfy the condition $\mathbf{n}_{i+1} \cdot \mathbf{r}(\mathbf{c})>0$. The existence of such a hyperplane permits one to trim the region $\boldsymbol{e}^{i} \cap H^{<}\left(\mathbf{n}_{i+1}, \gamma_{i+1}\right)$ from $\boldsymbol{e}^{i}$ to give the tighter bounding set $\boldsymbol{C}^{i+1}$. After $p$ stages, the bounding set $\boldsymbol{C}^{p}$ can be written as follows:

$$
\boldsymbol{e}^{p}=\left[\bigcap_{i=1}^{p} H^{\geq}\left(\mathbf{n}_{i}, \gamma_{i}\right)\right] \bigcap \boldsymbol{e}^{0}
$$

This means that the final bounding set $\boldsymbol{e}^{p}$ is the intersection of the original bounding set $\boldsymbol{e}^{0}$ with the each of the closed halfspaces $H^{\geq}\left(\mathbf{n}_{1}, \gamma_{1}\right), H^{\geq}\left(\mathbf{n}_{2}, \gamma_{2}\right), \ldots \ldots, H^{\geq}\left(\mathbf{n}_{p}, \gamma_{p}\right)$. If the set $\boldsymbol{C}^{0}$ is closed and bounded, then eqn (5.10) implies that $\boldsymbol{C}^{p}$ is also closed and bounded set. When $\boldsymbol{C}^{0}$ is convex (such as when $\boldsymbol{C}^{0}$ is the polyhedral set representing compositions that are stoichiometrically compatible with the feed), $\boldsymbol{e}^{p}$ is also convex. 


\subsection{Mathematical justification for the technique}

In this section our goal is to justify the claims made in Section 5.2. Let

$\boldsymbol{e}$ be a convex, closed and bounded set in $\mathbb{R}_{+}^{N}$ that contains all the compositions attainable by reaction and mixing (the set might contain additionally compositions that are not attainable) from a feed stream of composition $\mathbf{c}^{f}$. Suppose one can find a certain hyperplane $H^{=}(\mathbf{n}, \gamma)$ that divides the set into two regions, one region containing the feed composition and the other region having the property that each of its nonequlibrium compositions has a species-formation-rate vector associated with it that points toward the region containing the feed stream composition. Stated more formally, suppose that one can find a certain hyperplane $H^{=}(\mathbf{n}, \gamma)$ that satisfies the following two conditions: (1) the feed composition $\mathbf{c}^{f}$ lies in the halfspace defined by $H^{2}(\mathbf{n}, \gamma)$ and (2) for all nonequilibrium composition vectors in the set $\mathfrak{e} \cap H^{\leq}(\mathbf{n}, \gamma)$, it is true that $\mathbf{n} \cdot \mathbf{r}(\mathbf{c})>0$. Based on this supposition, the argument stated in the prior section makes the claim that no composition in $\boldsymbol{e} \cap H^{<}(\mathbf{n}, \gamma)$ can be the effluent stream composition of any steadystate reactor design that allows reactions and mixing alone and has a feed stream composition $\mathbf{c}^{f}$. As a result, one can remove this set to give a tighter bounding set $\left(\boldsymbol{e} \cap H^{2}(\mathbf{n}, \gamma)\right)$ on attainable compositions for the pure reactor synthesis problem.

The proof given here is adapted from Abraham and Feinberg [85] and is a proof by contradiction. Suppose that, contrary to the claim made earlier, there exists a steady-state process design involving reaction and mixing alone, that has a single feed stream of composition $\mathbf{c}^{f}$ and realizes within it a composition $\mathbf{c}^{\prime}$ that is a member of 
$\boldsymbol{e} \cap H^{<}(\mathbf{n}, \gamma)$. The goal here is to show that this supposition will lead to a contradiction.

The physical entities that constitute this process design will be collectively referred to as the system hereafter.

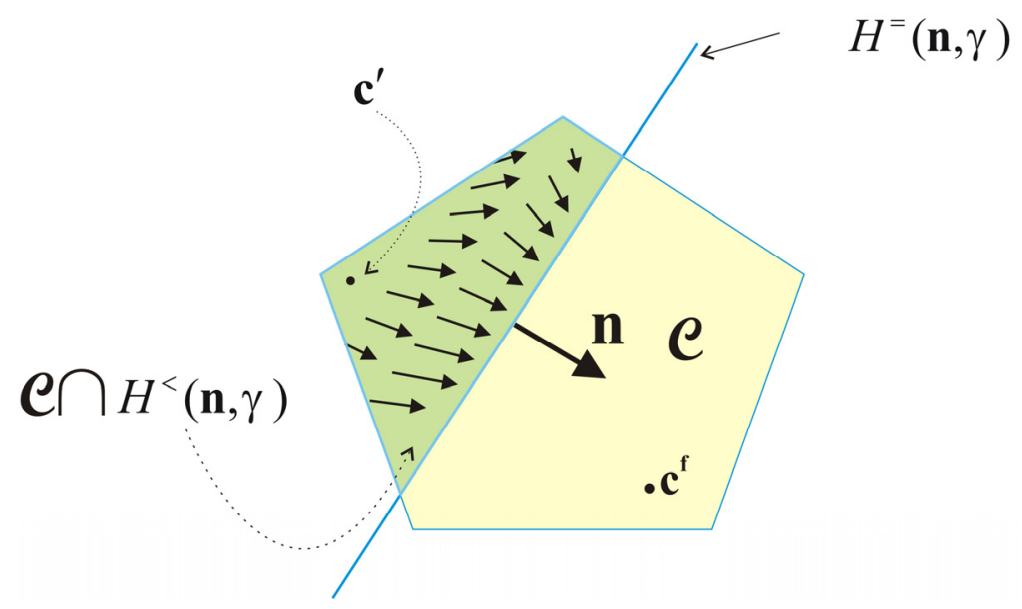

Figure 5.5 A hyperplane dividing the current composition set into two parts: one part containing the feed and in the other part all the rate vectors (shown as small directional arrows) at nonequilibrium compositions point toward the side containing the feed

One can define a test subsystem as a part of the system that contains only (and no other) compositions that lie in the set $\mathfrak{e} \cap H^{\leq}(\mathbf{n}, \gamma)$. Since $\mathbf{c}^{\prime} \in \boldsymbol{e} \cap H^{<}(\mathbf{n}, \gamma)$, such a composition will only appear in the test subsystem. One can write a molar balance for the test subsystem taking into account all the streams that flow away from and flow into it from other parts of the system. The streams that flow into the test subsystem might have all or part of the feed stream flowing into it while the streams that flow out of the test subsystem might flow to the remainder of the system or to the external world as an effluent.

As part of a molar balance, one needs to account for the chemical reactions that occur in the reactors that physically reside in the test subsystem. It is 
necessary for reactions to occur within the test subsystem otherwise the composition $\mathbf{c}^{\prime}$ might not be realized within it for the following reason: If there are no chemical reactions occurring in the test subsystem then all compositions that are in it must arise from mixing of streams from other parts of the system. These streams from other parts of the system must have mixture compositions that reside in $\mathfrak{e} \cap H^{2}(\mathbf{n}, \gamma)$ (a consequence of the definition of the test subsystem). Since the set $\boldsymbol{C} \cap H^{\geq}(\mathbf{n}, \gamma)$ is convex, the mixing of streams having compositions residing in this set results in a mixture whose composition also resides in the same set. This implies that without the occurrence of reactions in the test subsystem, the composition $\mathbf{c}^{\prime} \in \boldsymbol{e} \cap H^{<}(\mathbf{n}, \gamma)$ cannot be realized. For the composition $\mathbf{c}^{\prime}$ to be realized in the test subsystem, it must be achieved through reactors in the test subsystem that exhibit nonequlilibrium compositions otherwise it would have the same outcome as the absence of reactors.

Since the requirement is that $\mathbf{c}^{\prime}$ be a member of $\boldsymbol{e} \cap H^{<}(\mathbf{n}, \gamma)$, it must be true that at least one composition in a reactor in the test subsystem must also be a member of $\boldsymbol{e} \cap H^{<}(\mathbf{n}, \gamma)$ (the test subsystem is defined as all compositions that only lie in $\left.\boldsymbol{e} \cap H^{\leq}(\mathbf{n}, \gamma)\right)$ due to the following reason: If $\mathbf{c}^{\prime}$ itself is not a composition present in one of the reactors in the test subsystem, then it is a composition formed through the mixing of two streams from reactors within the test subsystem . If all the reactors in the test subsystem had effluent compositions in the set $\boldsymbol{e} \cap H^{=}(\mathbf{n}, \gamma)$, then due to convexity of that set, $\mathbf{c}^{\prime}$ would also lie in $\boldsymbol{e} \cap H^{=}(\mathbf{n}, \gamma)$ which is contradictory to what has been supposed earlier. 
The molar balance about the test subsystem can be written as follows:

$$
\mathbf{F}^{\text {out }}-\mathbf{F}^{\text {in }}=\mathbf{R}
$$

where $\mathbf{F}^{\text {out }}=\left[F_{1}^{\text {out }}, F_{2}^{\text {out }}, \ldots \ldots, F_{N}^{\text {out }}\right]$ is the vector representing the total molar flow rates of the $N$ chemical species in all the effluents flowing out of the test subsystem, $\mathbf{F}^{\text {in }}=\left[F_{1}^{\text {in }}, F_{2}^{\text {in }}, \ldots ., F_{N}^{\text {in }}\right]$ is the vector representing the total molar flow rates of the $N$ chemical species from all streams flowing into the test subsystem, and $\mathbf{R}=\left[R_{1}, R_{2}, \ldots \ldots, R_{N}\right]$ is the vector representing the molar production rates for the $N$ chemical species due to chemical reactions occurring everywhere in the test subsystem. Further analysis of equation (5.11) will lead to the proof that is being sought here. Let $v>0$ be the (steady-state) total volumetric flow rate of (incompressible) streams leaving the test subsystem. One can define $\mathbf{c}^{\text {out }}:=\mathbf{F}^{\text {out }} / v$, where $\mathbf{c}^{\text {out }}$ can be interpreted to be the composition that might be obtained by mixing all the effluent streams exiting the test subsystem in proportion to their individual volumetric flow rates. It is clear that $\mathbf{c}^{\text {out }}$ will be a convex combination (when there is more than one exiting stream) of the individual stream compositions, and since all compositions inside the test subsystem lie in the set $\boldsymbol{e} \cap H^{\leq}(\mathbf{n}, \gamma)$, hence $\mathbf{c}^{\text {out }}$ will lie in the convex set given by $\mathfrak{e} \cap H^{\leq}(\mathbf{n}, \gamma)$. Therefore, one can write:

$$
\begin{aligned}
& \mathbf{F}^{\text {out }}=v \mathbf{c}^{\text {out }} \\
& \mathbf{n} \cdot \mathbf{c}^{\text {out }} \leq \gamma
\end{aligned}
$$

Similarly, we can define $\mathbf{c}^{\text {in }}$ in terms of $\mathbf{F}^{\text {in }}$ and $v$ since the volumetric flow rates of streams entering the test subsystem are the same as those leaving the subsystem. If 
$\mathbf{c}^{\text {in }}:=\mathbf{F}^{\text {in }} / v$, then $\mathbf{c}^{\text {in }}$ can be interpreted to be the composition obtained by mixing all the streams entering the test subsystem in proportion to their individual volumetric flow rates. Stated more formally, $\mathbf{c}^{\text {in }}$ will be a convex combination of the compositions in the different streams that enter the test subsystem from the rest of the system. Since all the composition vectors outside the test subsystem but inside the system reside in the set given by $\boldsymbol{e} \cap H^{\geq}(\mathbf{n}, \gamma)$, then due to convexity of the set, $\mathbf{c}^{\text {in }}$ will also reside in it. Hence we can write:

$$
\begin{gathered}
\mathbf{F}^{\text {in }}=v \mathbf{c}^{\text {in }}, \\
\mathbf{n} \cdot \mathbf{c}^{\text {in }} \geq \gamma .
\end{gathered}
$$

The vector $\mathbf{R}$ was defined earlier as the vector of molar production rates of the chemical species due to reactions occurring everywhere in the test subsystem. The test subsystem is presumed to contain reactors and mixers in various configurations. Let the total effective volume of all the reactor components in the test subsystem be $V$. One can define $\overline{\mathbf{r}}:=\mathbf{R} / V$, where $\overline{\mathbf{r}}$ can be interpreted as the vector of per-unit-volume production rates of the $N$ species averaged over the summation of the volume of reactor components. Using some of the ideas discussed by Feinberg and Ellison [81], it is possible to see that $\overline{\mathbf{r}}$ is a convex combination of the species-formation-rate vectors that appear in the reactors within the test subsystem. More precisely, $\overline{\mathbf{r}}$ is a volume-weighted average of the species-formation-rate vectors exhibited in the reactor components of the test subsystem.

By supposition, all compositions within the reactor components of the test subsystem reside in the set $\boldsymbol{e} \cap H^{\leq}(\mathbf{n}, \gamma)$ and hence all nonequilibrium compositions 
exhibited within those reactor components satisfy the condition that $\mathbf{n} \cdot \mathbf{r}>0$. It was argued earlier why nonequilibrium compositions must be exhibited in the reactor components of the test subsystem and hence it follows that $\mathbf{n} \cdot \overline{\mathbf{r}}>0$ which implies that

$$
\mathbf{n} \cdot \mathbf{R}>0
$$

We can simplify equation (5.11) based on equations (5.12) and (5.14) to obtain the following equation:

$$
v\left(\mathbf{c}^{\text {out }}-\mathbf{c}^{\text {in }}\right)=\mathbf{R} .
$$

We perform a dot product of both sides of equation (5.17) with the vector $\mathbf{n}$ to obtain the following equation:

$$
v\left(\mathbf{c}^{\text {out }}-\mathbf{c}^{\text {in }}\right) \cdot \mathbf{n}=\mathbf{R} \cdot \mathbf{n} .
$$

By combining equations (5.13) and (5.15) we write:

$$
\left(\mathbf{c}^{\text {out }}-\mathbf{c}^{\text {in }}\right) \cdot \mathbf{n} \leq 0 .
$$

Upon closer inspection of equation (5.18) one can see that the left hand side of the equation will be governed by the inequality given in equation (5.19) while the right hand side of the equation will be governed by the inequality given in equation (5.16) and inequalities that govern the two sides do not agree. Hence the contradiction leading to the proof has been shown: Absolutely no steady-state design that employs reaction and mixing alone can have a feed stream composition $\mathbf{c}^{f}$ and have an output stream whose composition is $\mathbf{c}^{\prime}$ residing in $\boldsymbol{e} \cap H^{<}(\mathbf{n}, \gamma)$. 


\subsection{Treatment of multiple feed stream systems}

If there are $q$ feed streams, each having a different composition

$\mathbf{c}^{f_{1}}, \mathbf{c}^{f_{2}}, \ldots . ., \mathbf{c}^{f_{q}}$ then at every bound trimming step one checks for the following : a newly introduced hyperplane performs the following on the current bounding composition set, (1) all the feed stream compositions must reside on one side of the hyperplane and (2) the species-formation-rate vector computed for every nonequilibrium composition on the other side of the hyperplane points to the side containing all the multiple feed streams. Repeated trimming of the bounds using this modified condition will give tight outer bounds on the attainable region for the system with multiple feed streams. 


\section{CHAPTER 6}

\section{IMPLEMENTATION TECHNIQUES}

The goal of this chapter is to describe the algorithms and computing tools used in implementing the method of bounding hyperplanes on a personal computer. As far as we know, there is no single established computational technique that can perform the required computations for the method in its entirety. Therefore, various algorithms and computational tools will be described that aid in implementing the method. We do not fully know if the techniques described here are the best or the fastest available but the goal is to describe the experiences with various algorithms that were developed as part of the research. After introducing some preliminaries, we will discuss two approaches to implementation: a crude approach using "Maple" and a refined approach using "Solver DLL”.

\subsection{Preliminary ideas about polyhedral computation}

Let $\mathbb{R}^{N}$ be the usual vector space of $\mathrm{N}$-tuples. Any vector residing in it, say $\mathbf{x}$, will have the form $\mathbf{x}=\left[x_{1}, x_{2}, \ldots, x_{N}\right]$. In Chapter 5, we defined the closed halfspace $H^{\geq}(\mathbf{n}, \gamma)$ as: 


$$
H^{\geq}(\mathbf{n}, \gamma):=\left\{\mathbf{x} \in \mathbb{R}^{N}: \mathbf{n} \cdot \mathbf{x} \geq \gamma\right\}
$$

where $\mathbf{n}$ is a unit vector perpendicular to the hyperplane $H^{=}(\mathbf{n}, \gamma)$. If

$\mathbf{n}:=\left[n_{1}, n_{2}, \ldots \ldots, n_{N}\right]$, then the relation satisfied by all vectors in the closed halfspace

$H^{\geq}(\mathbf{n}, \gamma)$ might also be written as

$$
\mathbf{n} \cdot \mathbf{x}=\left[n_{1}, n_{2}, \ldots . . n_{N}\right] \cdot\left[x_{1}, x_{2}, \ldots . ., x_{N}\right]=n_{1} x_{1}+n_{2} x_{2}+\ldots . .+n_{N} x_{N} \geq \gamma
$$

A subset of $\mathbb{R}^{N}$ is called a convex polyhedron if it is the set of solutions to a finite number of linear inequalities [86]. A subset of $\mathbb{R}^{N}$ is called a convex polytope if it is a convex polyhedron and bounded.

A convex polytope has the property that it can be represented by either a finite number of linear inequalities or a finite number of extreme points [87]. A vector $\mathbf{x}$ is an extreme point of convex set $\boldsymbol{P}$ in $\mathbb{R}^{N}$ if $\mathbf{x}$ is a member of $\boldsymbol{P}$ and cannot be written as a positive convex combination of two distinct points in $\boldsymbol{P}$. That is, if $\mathbf{x}$ is a member of $\boldsymbol{P}$ and if $\mathbf{x}$ is an extreme point of $\boldsymbol{P}$ then for any choice of distinct $\left\{\mathbf{x}_{1}, \mathbf{x}_{2}\right\} \in \boldsymbol{P}$ we cannot find a value $\lambda$ that satisfies the following conditions:

$$
\begin{gathered}
\mathbf{x}=\lambda \mathbf{x}_{1}+(1-\lambda) \mathbf{x}_{2}, \\
0<\lambda<1 .
\end{gathered}
$$




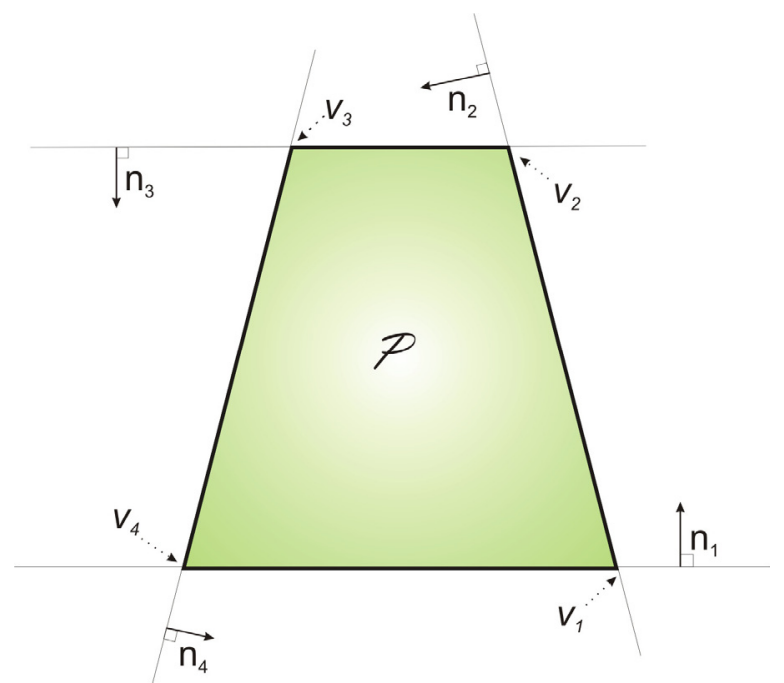

Figure 6.1 Example to describe the two representations that describe a convex set

Figure 6.1 shows an example of the dual representation of a convex set $\boldsymbol{P}$ in two dimensional space. Let $\mathbf{x}$ be any vector in $\mathbb{R}^{2}$. The set $\left\{\mathbf{v}_{1}, \mathbf{v}_{2}, \mathbf{v}_{3}, \mathbf{v}_{4}\right\}$ are four extreme points that represent the convex set $\boldsymbol{P}$. The same set is also represented by intersection of four halfspaces (as defined in Chapter 5) given by:

$$
\mathbf{n}_{1} \cdot \mathbf{x} \geq \gamma_{1}, \mathbf{n}_{2} \cdot \mathbf{x} \geq \gamma_{2}, \mathbf{n}_{3} \cdot \mathbf{x} \geq \gamma_{3}, \mathbf{n}_{4} \cdot \mathbf{x} \geq \gamma_{4},
$$

where $\mathbf{n}_{i}$ is a unit vector perpendicular to the hyperplane $\mathbf{n}_{i} \cdot \mathbf{x}=\gamma_{i}$ and $\gamma_{i}$ is the offset associated with the halfspace. Any vector $\mathbf{x}$ (residing in the two dimensional space) that satisfies all four inequalities resides in the set $\boldsymbol{P}$. Such a vector residing in $\boldsymbol{P}$, can also be represented as a convex combination of the four extreme points $\left\{\mathbf{v}_{1}, \mathbf{v}_{2}, \mathbf{v}_{3}, \mathbf{v}_{4}\right\}$.

An inequality belonging to a system of inequalities is termed redundant [87] if that inequality can be removed from the system without any changes to the polyhedral convex set described by the inequalities. A system of inequalities is said to have redundancy if one or more inequalities belonging to the system are redundant. If 
there are no redundant inequalities in a system of inequalities then it is categorized as a nonredundant system.

Figure 6.2 shows an example of a system of inequalities with a few redundant inequalities. The example is similar to the one shown in Figure 6.1. We are interested in the shaded region $\boldsymbol{P}$ that can be represented by extreme points or inequalities as described earlier. The extreme points that represent the set $\boldsymbol{P}$ are given by $\left\{\mathbf{v}_{1}, \mathbf{v}_{2}, \mathbf{v}_{3}, \mathbf{v}_{4}\right\}$ while the same set is given by the intersection of seven halfspaces:

$$
\mathbf{n}_{1} \cdot \mathbf{x} \geq \gamma_{1}, \mathbf{n}_{2} \cdot \mathbf{x} \geq \gamma_{2}, \mathbf{n}_{3} \cdot \mathbf{x} \geq \gamma_{3}, \mathbf{n}_{4} \cdot \mathbf{x} \geq \gamma_{4}, \mathbf{n}_{5} \cdot \mathbf{x} \geq \gamma_{5}, \mathbf{n}_{6} \cdot \mathbf{x} \geq \gamma_{6}, \mathbf{n}_{7} \cdot \mathbf{x} \geq \gamma_{7} .
$$

In this example, it is the case (at least pictorially) that the halfspaces corresponding to the normals $\mathbf{n}_{5}, \mathbf{n}_{6}$ and $\mathbf{n}_{7}$ can be removed from the system without any changes to the set $\boldsymbol{P}$. In the figure, the hyperplane corresponding to the normal $\mathbf{n}_{7}$ passes through the extreme point $\mathbf{v}_{4}$ but has no influence on the set $\boldsymbol{P}$ since it can be removed without consequence. The halfspaces corresponding to the normals $\mathbf{n}_{1}, \mathbf{n}_{2}, \mathbf{n}_{3}$ and $\mathbf{n}_{4}$ make up a minimal set that can describe the set $\boldsymbol{P}$ completely and are referred to as the extreme halfspaces of the set $\boldsymbol{P}$. These definitions will be invoked while deriving the equation for a new hyperplane at an extreme point later in this chapter. 


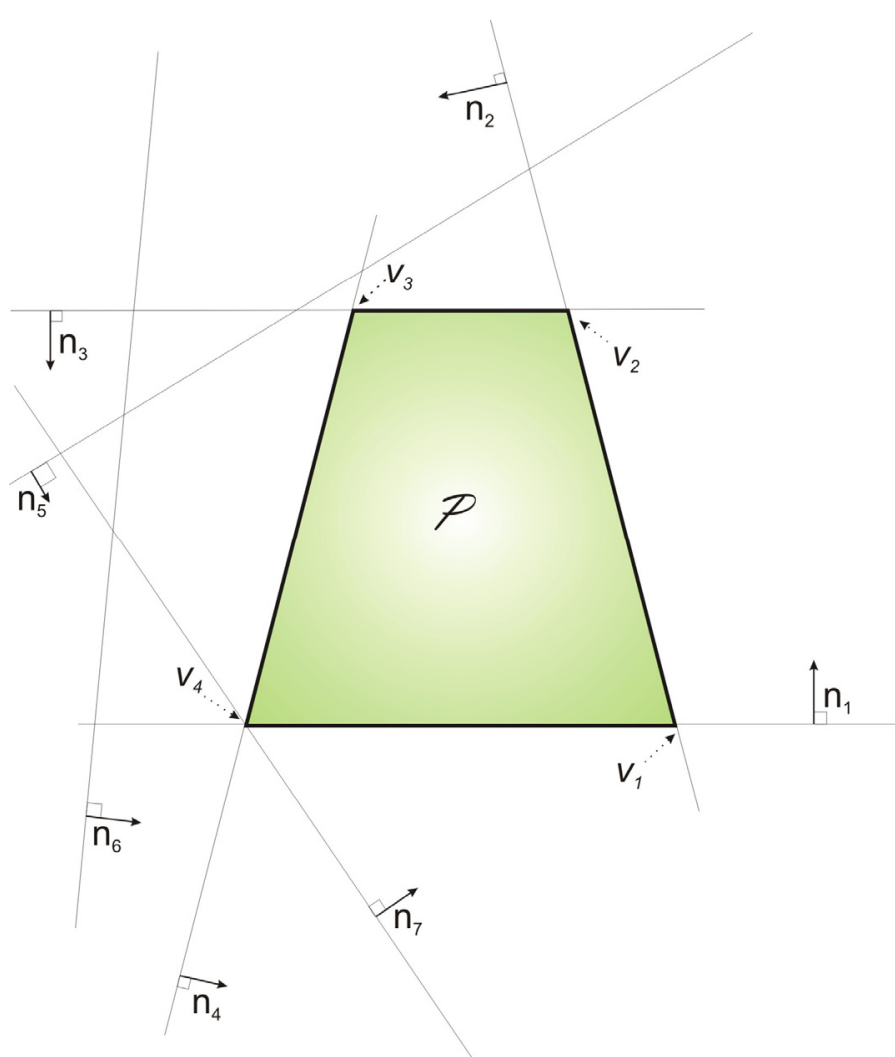

Figure 6.2 An example of redundant inequalities in a system of inequalities

The software implementation of the method of bounding hyperplanes often encounters redundant inequalities while working with different reaction networks. Removing redundancy is important, not only because it reduces computational load but also because this is important in the step where new hyperplanes are being introduced.

The concept of incidence is used later on in this chapter and is defined by Fukuda [89] as follows: An extreme point of a convex polytope is said to be incident with an inequality if the inequality is satisfied by equality (alone). In Figure 6.1, the extreme point $\mathbf{v}_{1}$ is incident with the inequalities $\mathbf{n}_{1} \cdot \mathbf{x} \geq \gamma_{1}, \mathbf{n}_{2} \cdot \mathbf{x} \geq \gamma_{2}$ because it satisfies the two equalities $\mathbf{n}_{1} \cdot \mathbf{x}=\gamma_{1}, \mathbf{n}_{2} \cdot \mathbf{x}=\gamma_{2}$. In other words, the vector $\mathbf{v}_{1}$ is a possible solution if we 
attempt to solve both the equations simultaneously [87] . An incidence map lists which equalities are incident at every extreme point. In Figure 6.2, the extreme point $\mathbf{v}_{4}$ is satisfied by the three equalities given by, $\mathbf{n}_{1} \cdot \mathbf{x}=\gamma_{1}, \mathbf{n}_{4} \cdot \mathbf{x}=\gamma_{4}, \mathbf{n}_{7} \cdot \mathbf{x}=\gamma_{7}$.

The cosine of the angle between the normals of two hyperplanes $\mathbf{n}_{1} \cdot \mathbf{x}=\gamma_{1}$ and $\mathbf{n}_{2} \cdot \mathbf{x}=\gamma_{2}$ residing in $\mathbb{R}^{N}$ can be defined as

$$
\cos (\theta):=\frac{\mathbf{n}_{1} \cdot \mathbf{n}_{2}}{\left\|\mathbf{n}_{1}\right\| \cdot\left\|\mathbf{n}_{2}\right\|}, 0 \leq \theta \leq \pi
$$

where $\left\|\mathbf{n}_{1}\right\|=\sqrt{\mathbf{n}_{1} \cdot \mathbf{n}_{1}}$ is the Euclidean norm of the vector $\mathbf{n}_{1}$. We will refer to $\cos (\theta)$ as an angle parameter that describes the relative position of one hyperplane with respect to the other at the intersection. To make this concept clear, we resort to looking at the angle parameter in $\mathbb{R}^{2}$ for two pairs of intersecting hyperplanes with outward normals $\mathbf{n}_{1}$ and $\mathbf{n}_{2}$. In Figure 6.3, the first pair of hyperplanes meet sharply, i.e. the extreme point is pointed or sharp edged, and $\theta_{1}$ is the angle between their normals. The second pair of hyperplanes meet smoothly, i.e. the extreme point is not sharp edged, and $\theta_{2}$ is the angle between their normals. Our definition of sharpness and smoothness at the intersection of two hyperplanes will be very coarse and the terms are meant to be used in a relative manner. In other words, our desire is to simply define a characteristic that lets us say that one extreme point is sharper than the other, or say that one extreme point is smoother than the other. These ideas can be extended to higher dimensions also. For example in $\mathbb{R}^{3}$, if we have 3 hyperplanes intersecting then a "sharp" extreme point would be one where the angle between any pair of normals is relatively large. 

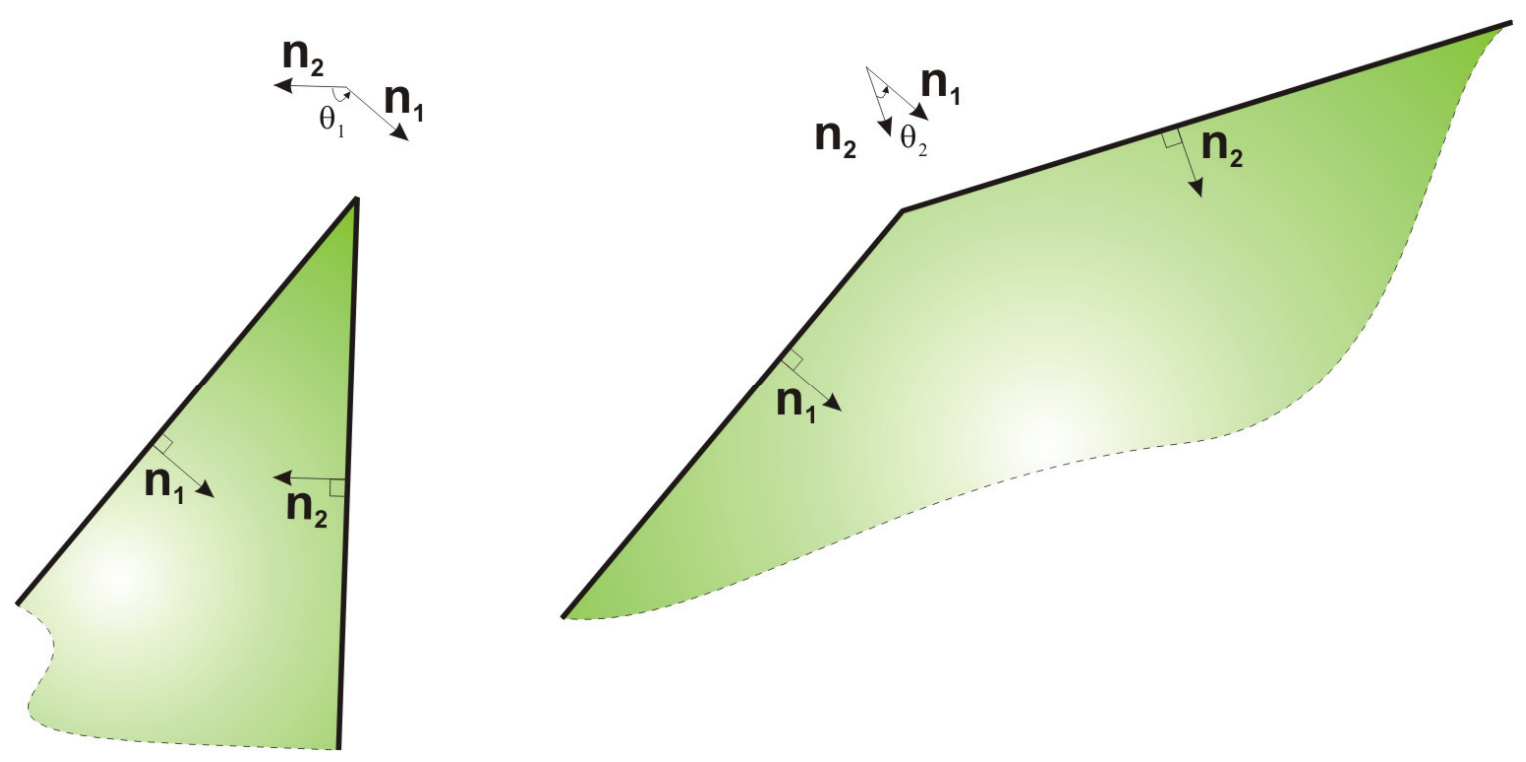

Figure 6.3 Hyperplanes with different angle parameters

In Figure 6.3, the sharper extreme point has a larger angle $\theta_{1}$ between the normals as compared to the smoother extreme point with angle $\theta_{2}$ between the normals. When the normals are at an angle closer to zero then the cosine of that angle tends to be closer to 1 . When the normals are at an angle closer to 180 degrees then the cosine of that angle tends to be closer to -1 . This means that the angle parameter computed at a sharper edge will be smaller than that computed at a smoother edge. These ideas will be invoked later on in this chapter where "smarter" techniques to create outer bounds on the attainable region are discussed.

We define hyperplane variance, analogous to angle parameter at an extreme point, but more suitable for dealing with hyperplanes in higher dimensions. 
Suppose that there are $k$ hyperplanes intersecting at an extreme point and their outward unit normals are $\mathbf{n}_{1}, \mathbf{n}_{2}, \ldots . ., \mathbf{n}_{k}$. We define a preliminary average, $\mathbf{n}_{\text {avg }}$, as

$$
\mathbf{n}_{\text {avg }}:=\frac{1}{k} \sum_{i=1}^{k} \mathbf{n}_{i}
$$

and a normalized average, $\overline{\mathbf{n}}$, is defined as

$$
\overline{\mathbf{n}}:=\frac{\mathbf{n}_{\text {avg }}}{\left\|\mathbf{n}_{\text {avg }}\right\|}, \text { where }\left\|\mathbf{n}_{\text {avg }}\right\|=\sqrt{\mathbf{n}_{\text {avg }} \cdot \mathbf{n}_{\text {avg }}} .
$$

The hyperplane variance at that extreme point, $\sigma^{2}$, can be defined as

$$
\sigma^{2}:=\frac{1}{k} \sum_{i=1}^{k}\left(\left\|\mathbf{n}_{i}-\overline{\mathbf{n}}\right\|\right)^{2}
$$

If we define $\cos \theta\left(\mathbf{n}_{i}, \overline{\mathbf{n}}\right)$ as the angle parameter between $\mathbf{n}_{i}$ and $\overline{\mathbf{n}}$ then by invoking the definition of the angle parameter, we can show that $\sigma^{2}$ ranges from a high value of 4 to a low value of 0 . A high value of the hyperplane variance, means that the normals deviate from the normalized average by a large value. For the example in Figure 6.3, the hyperplanes that intersect at a sharper edge tend to have normals that deviate from the average of the normals by a larger extent.

\subsection{Maple implementation and algorithms}

Maple is an advanced mathematical and analytical software published by Maplesoft. Most of the initial research for this thesis was done on this platform due to the ease and speed with which Maple permits one to implement complex algorithms. The availability of built-in user functions with a variety of options makes this possible. This 
section will be used to describe the algorithms that were used in Maple for computation associated with the method of bounding hyperplanes.

As in the previous chapter, it is assumed that a chemical reaction network with $N$ species $\left(\mathrm{A}_{1}, \mathrm{~A}_{2}, \ldots . \mathrm{A}_{\mathrm{N}}\right)$ is given along with kinetics and that reactions occur under isothermal conditions. A single feed stream of known composition is available and mixtures are homogeneous and of uniform density throughout the system.

Given a reaction network and a feed stream composition, one can compute the stoichiometric constraints of the reaction system corresponding to that feed composition (as described in Chapter 4). The stoichiometric constraints bound the set of all compositions that are stoichiometrically compatible with the feed and typically such a set will be a convex polytope [85] in $\mathbb{R}_{+}^{N}$ (the positive orthant of an $N$-dimensional composition vector space) .

Such a polyhedron can be described by a system of inequalities as follows:

$$
\begin{aligned}
& a_{1}^{L} c_{1}+a_{2}^{L} c_{2}+\ldots . .+a_{N}^{L} c_{N} \leq b^{L}, \\
& L=1,2, \ldots ., P
\end{aligned}
$$

where $c_{i}$ is a variable representing the concentration of species $\mathrm{A}_{\mathrm{i}}, a_{i}^{L}$ is the coefficient corresponding to the $i^{\text {th }}$ species and $L$ is the index identifying a single inequality constraint. 
We define the current polytope, $\mathscr{P}$, as the convex polytope defined by the bounding hyperplanes at the beginning of any iteration. Therefore, at the very beginning of all computations the stoichiometric constraints bound our current polytope. Methods to compute the stoichiometric constraints are described in Section 4.1 of Chapter 4 in great detail.

Besides the computation of stoichiometric constraints, other information we need to begin computation is the species-production-rate function. This is the $\mathbb{R}^{N}$ valued function such that

$$
\mathbf{r}(\mathbf{c})=\left[r_{1}(\mathbf{c}), r_{2}(\mathbf{c}), \ldots . ., r_{N}(\mathbf{c})\right],
$$

where $r_{i}(\mathbf{c})$ is the local production rate (in moles per unit volume) of species $A_{i}$ due to the occurrence of all reactions when the local composition is $\mathbf{c}$. In the examples of this thesis, the species-production-rate vectors are derived by assuming mass-action kinetics. The method of bounding hyperplanes does not preclude the use of user-defined reaction rate vectors, but the examples in this thesis use rate vectors that are derived assuming mass-action kinetics.

For the Van de Vusse reaction system described earlier,

$$
\begin{aligned}
& \underset{\mathrm{A}_{1} \rightarrow \mathrm{A}_{2}}{\stackrel{1}{\rightarrow}} \mathrm{A}_{4} \\
& 2 \mathrm{~A}_{1} \stackrel{10}{\rightarrow} \mathrm{A}_{3},
\end{aligned}
$$

we can write the species-production-rate vector in terms of the local species concentrations as follows:

$$
\mathbf{r}(\mathbf{c})=\left[-c_{1}-20 c_{1}^{2}, c_{1}-c_{2},-10 c_{1}^{2}, c_{2}\right] .
$$


At this point, we are ready to begin computation using the method of bounding hyperplanes. The entire algorithm used to implement the method in Maple is shown in Figure 6.5 and will be described more in detail in the following paragraphs. As mentioned earlier we begin with the stoichiometric constraints and the first step is the computing of extreme points. This was done using the program cdd distributed by Fukuda $[86,89]$. Details about the program are described in the appendix but the basic task it performs is the conversion of the description of a convex polyhedron in the form of inequalities to the extreme points description. For example, the stoichiometric constraints for the 3-d van de Vusse problem (where one is interested in the compositions of $\mathrm{A}_{1}, \mathrm{~A}_{2}$ and $\mathrm{A}_{3}$ alone) for a feed stream composition of $\mathbf{c}^{f}=[1,0,0]$ can be computed to be:

$$
\begin{aligned}
& c_{1} \geq 0, c_{2} \geq 0, c_{3} \geq 0 \\
& c_{1}+c_{2}+2 c_{3} \leq 1
\end{aligned}
$$

The corresponding extreme points for the polyhedron formed by these inequalities are found to be:

$$
[0,0,0],[1,0,0],[0,1,0],[0,0,0.5] \text {. }
$$




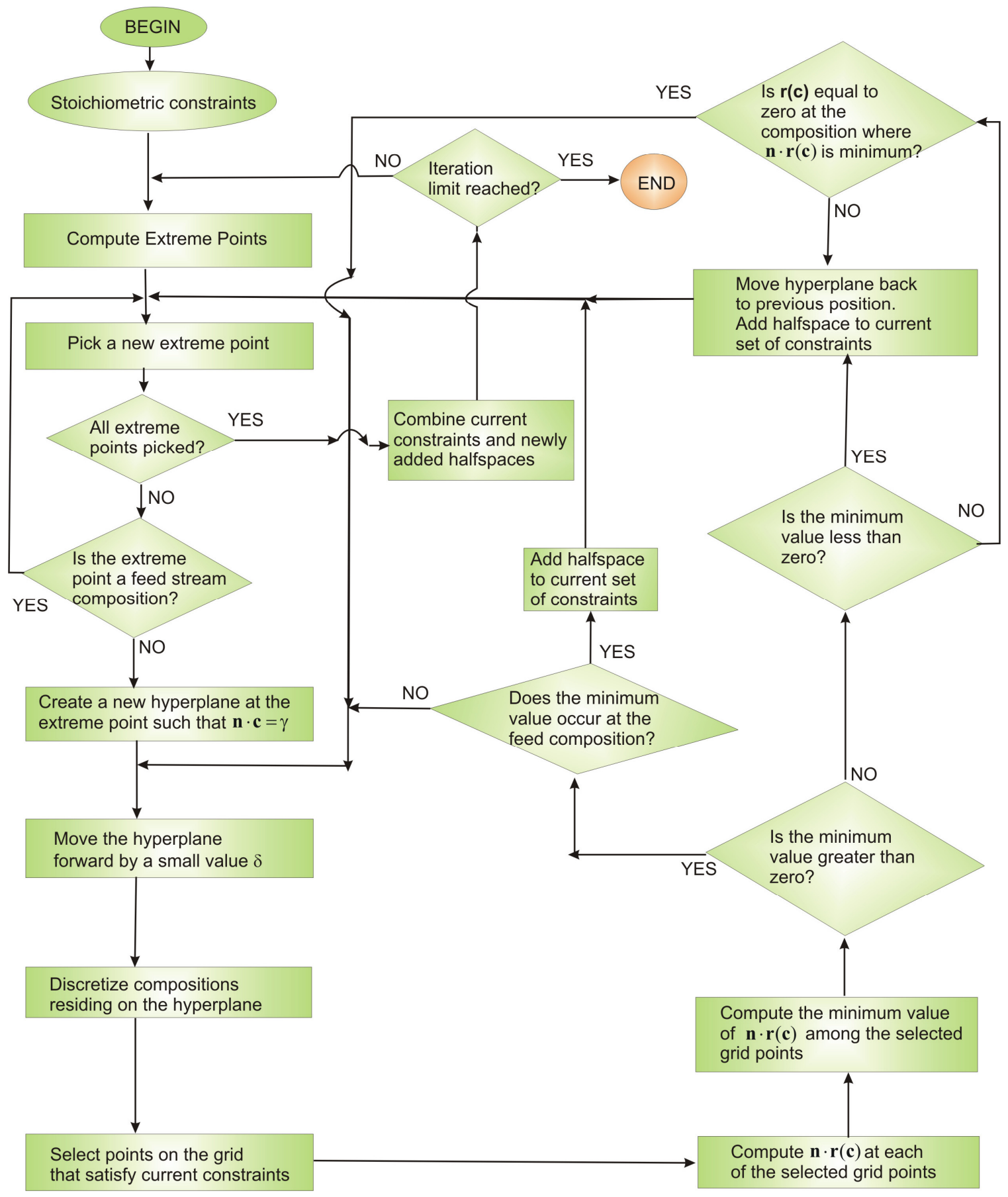

Figure 6.4 Algorithm for the Maple implementation of the method of bounding hyperplanes 
In the next computational step, we enter into a conditional loop where each extreme point is "visited" and computations are performed starting at these extreme points (as outlined in Figure 6.4).

If the extreme point picked coincides with the feed stream composition, then no computations are done at that extreme point. This is because the method of bounding hyperplanes requires that the feed stream composition lie entirely on one side of or reside on any newly introduced hyperplane meant to trim off compositions that are not feasible.

When the extreme point is not a feed stream composition, we can introduce a new hyperplane, initially passing through the extreme point. We cannot introduce any hyperplane at an extreme point because our choice is restricted to those that are supporting hyperplanes (see Section 5.1, page 63 for definition) for the current polytope (i.e. the newly introduced hyperplane cannot cut the current polytope.)

If we are at an extreme point representing the composition $\mathbf{c}^{*}$, we are interested in introducing a new hyperplane $\mathbf{n}^{*} \cdot \mathbf{c}=\gamma^{*}$ (conforming to the convention adopted in Section 5.1) where $\mathbf{n}^{*}$ is a unit normal orthogonal to the hyperplane and $\gamma^{*}$ is its offset.

There are two unknowns that need to determined in this step of the computation, $\mathbf{n}^{*}$ and $\gamma^{*}$. We begin by first computing $\mathbf{n}^{*}$ which in geometric terms is roughly the orientation of the hyperplane. Figure 6.5 shows a possible fragment (in $\mathbb{R}^{2}$ ) of the current polytope, and the figure will guide our discussions. 


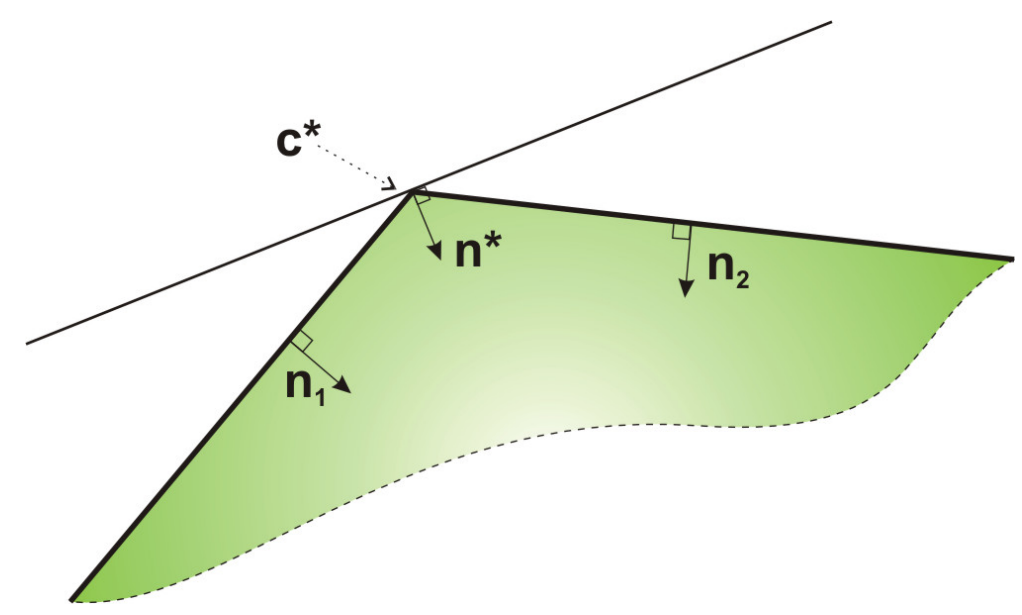

Figure 6.5 A fragment of the current polytope showing computation at an extreme point

If we pick $\mathbf{n} *$ to be a convex combination of normals of the hyperplanes incident at the extreme point $\mathbf{c}^{*}$, then we can write:

$$
\mathbf{n}^{*}:=\lambda \mathbf{n}_{1}+(1-\lambda) \mathbf{n}_{2} \text { where } 0<\lambda<1
$$

From the theory described in Chapter 5, we know that at the extreme point $\mathbf{c}^{*}$, it is true that:

$$
\mathbf{n}_{1} \cdot \mathbf{r}\left(\mathbf{c}^{*}\right) \geq 0 \text { and } \mathbf{n}_{2} \cdot \mathbf{r}\left(\mathbf{c}^{*}\right) \geq 0
$$

Moreover, if $\mathbf{r}\left(\mathbf{c}^{*}\right) \neq \mathbf{0}$ we must have either $\mathbf{n}_{1} \cdot \mathbf{r}\left(\mathbf{c}^{*}\right)>0$ or $\mathbf{n}_{2} \cdot \mathbf{r}\left(\mathbf{c}^{*}\right)>0$. This follows from the independence of the set $\left\{\mathbf{n}_{1}, \mathbf{n}_{2}\right\}$. Combining this and eqn (6.13) we can conclude that,

$$
\mathbf{n} * \mathbf{r}\left(\mathbf{c}^{*}\right)>0
$$

From equation (6.15) it is clear that the new hyperplane, calculated as shown in equation (6.13), might be instrumental in trimming the current bounds on the set of feasible compositions. 
Now that the value of $\mathbf{n}^{*}$ has been computed, the value of the offset $\gamma^{*}$ when the hyperplane passes through $\mathbf{c}^{*}$ can be computed by the simple relation:

$$
\mathbf{n} * \cdot \mathbf{c}^{*}=\gamma *
$$

At this point we have a hyperplane passing through the chosen extreme point, but the goal is to move the hyperplane (by changing the offset) as far as possible such that the following two conditions are satisfied: (1) The feed is on one side of or resides on the new hyperplane and (2) On the other side of the hyperplane and within the current polytope $(\mathscr{P})$, the compositions give rise to species production rate vectors that point to the side containing the feed.

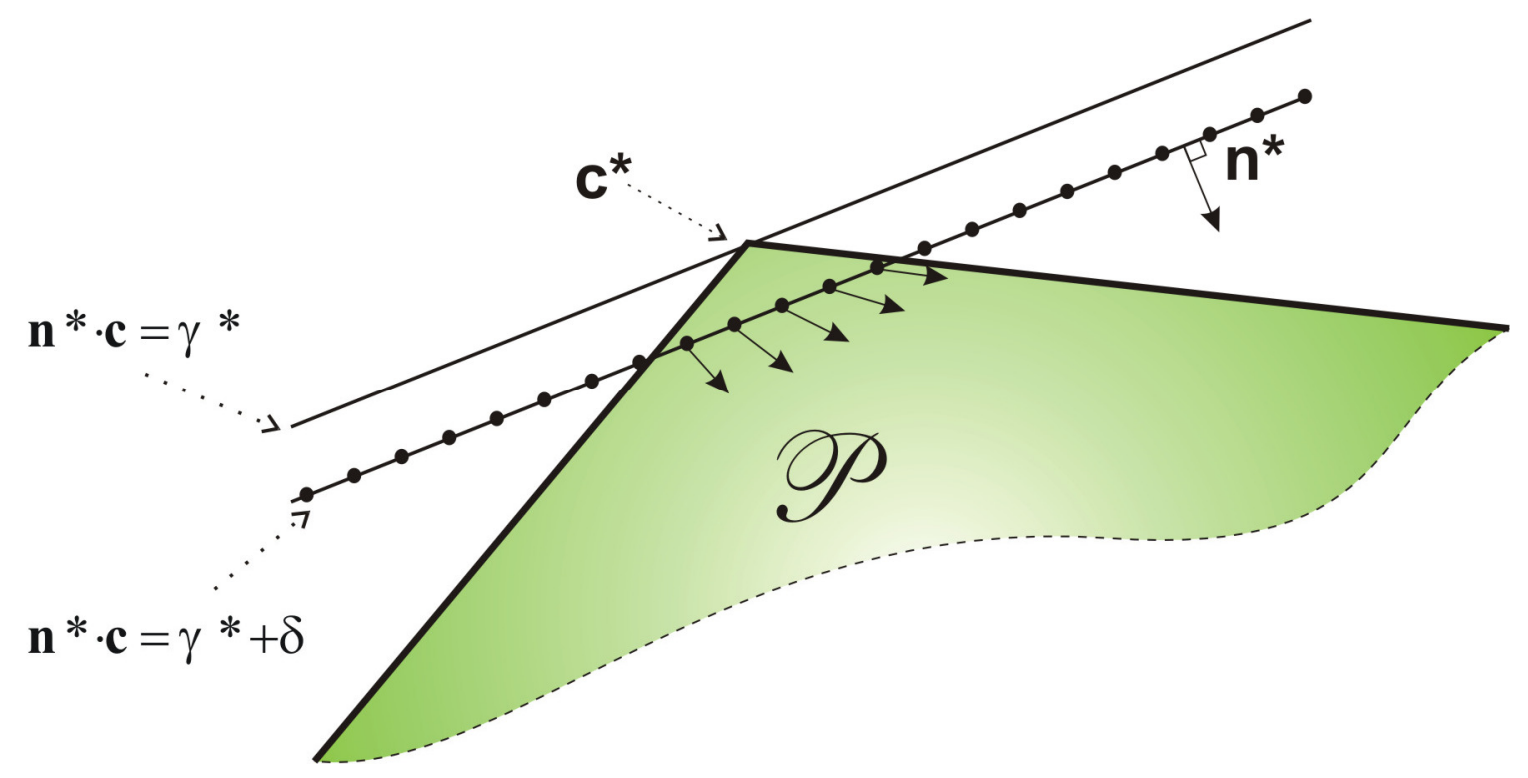

Figure 6.6 A fragment of the current polytope showing a new hyperplane being "moved inside" it.

Figure 6.6 shows the new hyperplane $\mathbf{n} * \mathbf{c}=\gamma^{*}$ being "moved" to a new position such that $\mathbf{n}^{*} \cdot \mathbf{c}=\gamma^{*}+\delta$ where $\delta$ is referred to as the hyperplane step size. For 
our computations, the value of the step size was chosen to be a small value, such as $10^{-3}$ based on experience with the computations. A smaller step size would increase the computational burden while a larger step size might cause erroneous results. The reasons for this will be explained later.

The next step is to find discrete values on the hyperplane where we can evaluate the species-formation-rate function. For a composition vector with $N$ components, one needs to assign $N-1$ component values based on a grid; then the $N^{\text {th }}$ component can be computed from the equation of the hyperplane. Discretization is necessary since every value in composition space cannot be evaluated from a practical viewpoint. A grid density can be defined as the number of partitions created in each component of the composition vector. A different grid density can be chosen for each component so that some species concentrations can be emphasized more than the other. A grid size is the size of the partition for a given component. It is computed by dividing each component of the composition vector based on the minimum/maximum value computed by stoichiometric considerations and the grid density chosen.

For example, assume that we choose a uniform grid density of 100 and that the composition vector has 3 components $\left[c_{1}, c_{2}, c_{3}\right]$ where the minimum values of the components are $[0,0,0]$ and the maximum values are $[1,0.5,1]$. Since there are 3 components, we create a grid using 2 components $c_{1}$ and $c_{2}$ and compute $c_{3}$ from the equation of the hyperplane. The grid size for $c_{1}$ will be $\Delta c_{1}=\frac{1}{100}$ and that for $c_{2}$ will be 
$\Delta c_{2}=\frac{0.5}{100}$ and we begin with the minimum values for each component. In this example, the sequence of $c_{1} c_{2}$ pairs can be evaluated by the following sequence:

$$
\left(\left(\left[i * \Delta c_{1}, j * \Delta c_{2}\right], j=1 . .100\right), i=1 . .100\right)
$$

where the square brackets represent the $c_{1} c_{2}$ pairs chosen for different integer values of $i$ and $j$ sequentially. Once these pairs are chosen, $c_{3}$ can be computed from the equation of the hyperplane to determine those composition vectors that reside on the hyperplane.

Figure 6.7 shows what the grid might look like for this example. Every point formed by the intersection of a vertical and horizontal line is a grid point or $c_{1} c_{2}$ pair.

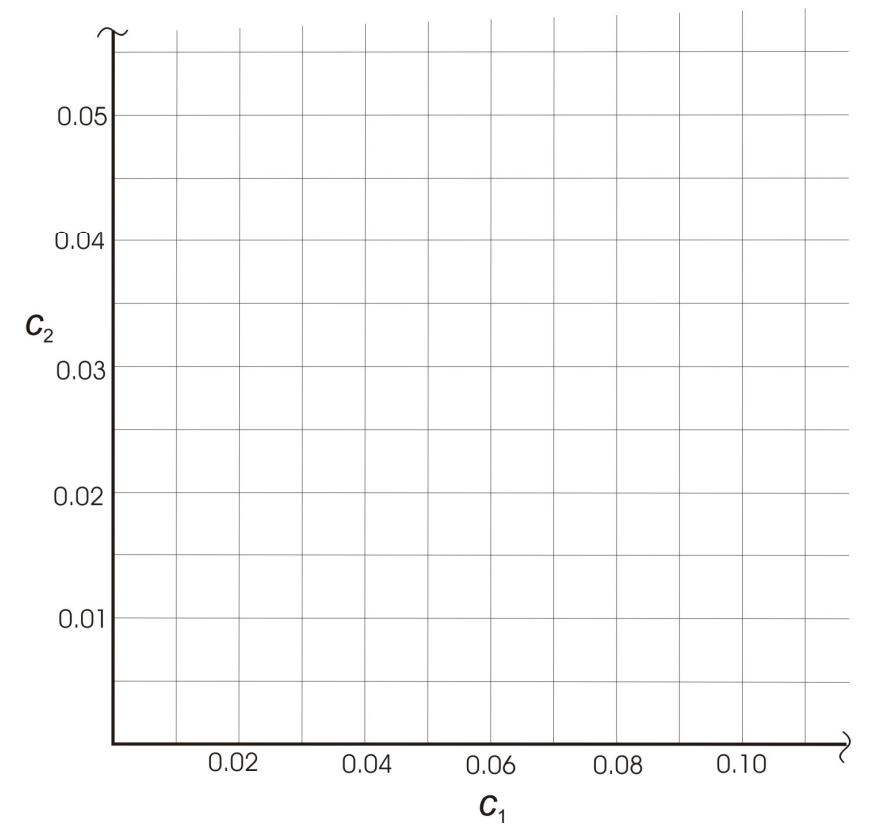

Figure 6.7 A grid to determine points residing on a hyperplane 
The next step in the computation involves determining which of the grid points need to be tested using the species-formation-rate function $\mathbf{r}(\mathbf{c})$. Since we know the inequalities that represent the current polytope $\mathscr{P}$, this step involves taking each grid point and checking if it satisfies all the inequalities (for example all the inequalities in equation 6.11). If it violates at least one inequality then that composition vector is discarded and the undiscarded composition vectors are called valid grid points. In other words, we are discarding those points that lie outside of $\mathscr{P}$.

It might happen that the value of the hyperplane step-size might be so small that no valid grid points are found. In this case the offset is increased further and the hyperplane is "moved" further until valid grid points are found.

Once a set of valid grid points is obtained, $\mathbf{r}(\mathbf{c})$ and $\mathbf{n} * \mathbf{r}(\mathbf{c})$ is computed at each of these points. If the computed values of $\mathbf{n} * \cdot \mathbf{r}(\mathbf{c})$ are all strictly greater than zero then the hyperplane is "moved" further by the hyperplane step size $\delta$. If there is at least one composition where the computed value of $\mathbf{n} * \mathbf{r}(\mathbf{c})$ is less than zero, then the hyperplane is "moved" back to its previous position. 


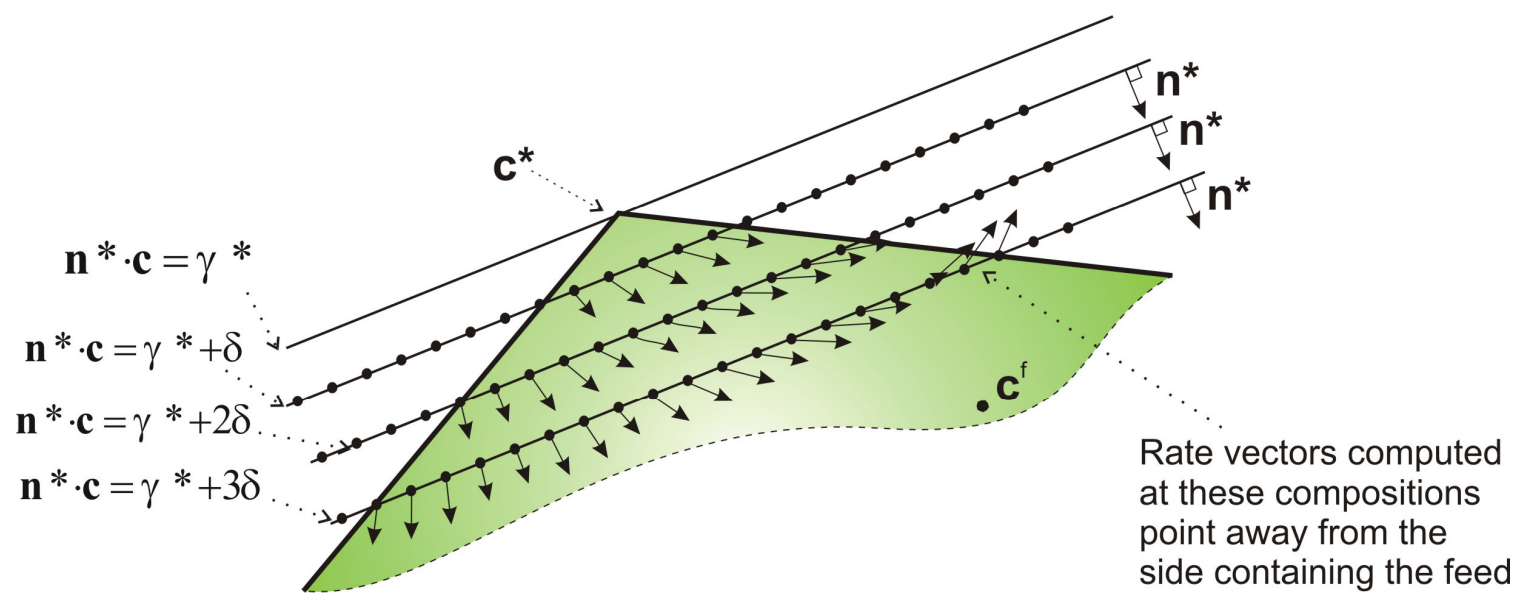

Figure 6.8 An example showing the creation of grid points and moving of a hyperplane

Figure 6.8 shows a fragment of a current polytope (that might be formed by stoichiometric constraints or otherwise) where we are interested in the extreme point $\mathbf{c}^{*}$ and a new hyperplane $\mathbf{n} * \mathbf{c}=\gamma^{*}$ (where $\mathbf{n} *$ is the normal perpendicular to the hyperplane) is introduced that coincides with this extreme point. The feed stream composition $\mathbf{c}^{f}$ resides inside the current polytope and on one side of the new hyperplane. Following the computational steps mentioned earlier, the hyperplane was "moved" forward by increasing the offset by a value $\delta$. Then grid points were created and these are represented as thick dots on the hyperplane. Then the rate vectors were computed for the valid grid points and these are shown as the small pointed arrow emanating from the thick dots.

When the offset is equal to $\gamma^{*}+\delta$, two conditions are satisfied: firstly the feed stream is on one side of the hyperplane and secondly, all valid grid point compositions give rise to species-formation-rate vectors such that $\mathbf{n} * \cdot \mathbf{r}(\mathbf{c})>0$ (i.e. they point to the side containing the feed). The offset is increased to $\gamma^{*}+2 \delta$ which moves the 
hyperplane forward. After determining the valid grid points and computing the speciesproduction-rate vectors, we see that the feed stream is still on one side of the hyperplane and all the rate vectors computed, point to the side containing the feed.

The offset is increased to $\gamma^{*}+3 \delta$ (see Figure 6.9) which moves the hyperplane forward. After determining the valid grid points and computing the speciesproduction-rate vectors, we see that the feed stream is still on one side of the hyperplane but some of the rate vectors computed do not point to the side containing the feed but away from it. In other words some valid grid points give rise to rate vectors such that $\mathbf{n}^{*} \mathbf{r}(\mathbf{c})<0$, which is not acceptable. This means we cannot "move" the hyperplane to the offset $\gamma^{*}+3 \delta$ because it violates one of the two conditions required by the method of bounding hyperplanes.

Hence the new hyperplane at an offset of $\gamma^{*}+2 \delta$ is the most we can move the hyperplane with normal $\mathbf{n} *$ while satisfying the two conditions for the method and the current constraints. We then add the halfspace corresponding to that hyperplane to our current list of constraints and move on to the next extreme point. Note that the new halfspace might also create new extreme points if they are combined with the existing constraints but this is done only after all the extreme points at the beginning of the computation have been examined. The reason for this is that the step to compute extreme points for a system of inequalities involves the most computational load and hence it is beneficial to minimize computing extreme points as much as possible.

If the lowest computed value of $\mathbf{n}^{*} \cdot \mathbf{r}(\mathbf{c})$ equals zero, then we need to confirm independently if the species-production-rate vector computed at that grid point 
equals zero or in other words check if $\mathbf{r}(\mathbf{c})=\mathbf{0}$. If this is true, then we can continue increasing the offset for the hyperplane and hence move it further.

$$
\text { If } \mathbf{r}(\mathbf{c}) \neq \mathbf{0} \text { and } \mathbf{n} * \mathbf{r}(\mathbf{c})=\mathbf{0} \text { then no further movement of the hyperplane }
$$

might be possible and the halfspace corresponding to the hyperplane and the current offset is added to the list of current constraints. This is done because the hyperplane does not violate either of the two conditions but it is highly probable that if we increase the offset there will be compositions such that $\mathbf{n} * \mathbf{r}(\mathbf{c})<\mathbf{0}$ and this violates one of the two conditions. From a geometric viewpoint, the fact that $\mathbf{r}(\mathbf{c}) \neq \mathbf{0}$ and $\mathbf{n} * \cdot \mathbf{r}(\mathbf{c})=\mathbf{0}$ means that the two vectors are perpendicular to each other and there is a high probability that compositions nearby where $\mathbf{r}(\mathbf{c}) \neq \mathbf{0}$ might give rise to rate vectors that point away from the side containing the feed.

After making a decision about whether to discard or accept the new hyperplane, we move to the next extreme point and the entire process is repeated until all extreme points have been examined for possible introduction of hyperplanes. After all the extreme points are examined the next step is to combine the new constraints with the existing constraint and use cdd to find a possibly new set of extreme points. We again examine each new extreme point, introduce hyperplanes and attempt to move them as before. Every computation that introduces a new hyperplane can give rise to more extreme points and every new extreme point can possibly introduce a new hyperplane in the next iteration.

There are various parameters that affect the number of hyperplanes that can be introduced and the trimming away of infeasible compositions. For the Maple 
implementation, these parameters are predominantly the grid density and the step size (as described earlier). For fixed values of these parameters, the iterative process described continues until a limit that is set on iterations is reached or there are no new (trimming) hyperplanes that can be introduced (for the parameters chosen.) In the latter case, parameters can be changed and the computation continued so that we can add more hyperplanes and attempt to remove more kinetically infeasible compositions.

The results using this method in Maple are discussed in the next chapter, but they served mostly as a tool for testing various computational techniques to implement the method of bounding hyperplanes. As experimentation with the techniques progressed various drawbacks about this method became evident.

Firstly, the discretization of grid points on a hyperplane were found to be problematic in that we would frequently "miss" composition vectors of interest during discretization. In other words, a composition vector of interest, say where the rate vector would be such that $\mathbf{n}^{*} \cdot \mathbf{r}(\mathbf{c})<\mathbf{0}$, might lie between two sequential valid grid points that have $\mathbf{n}^{*} \cdot \mathbf{r}(\mathbf{c})>\mathbf{0}$.

A slightly different situation that could arise is shown in Figure 6.9 where we have a hyperplane being moved into the current polytope. The feed stream is on one side of the hyperplane and all the grid point compositions within the current constraints give rise to rate vectors that point to the side containing the feed. Based on the method described above the hyperplane can be moved ahead. However, it might be possible that there exists a composition inside the current polytope between two grid points, one valid, 


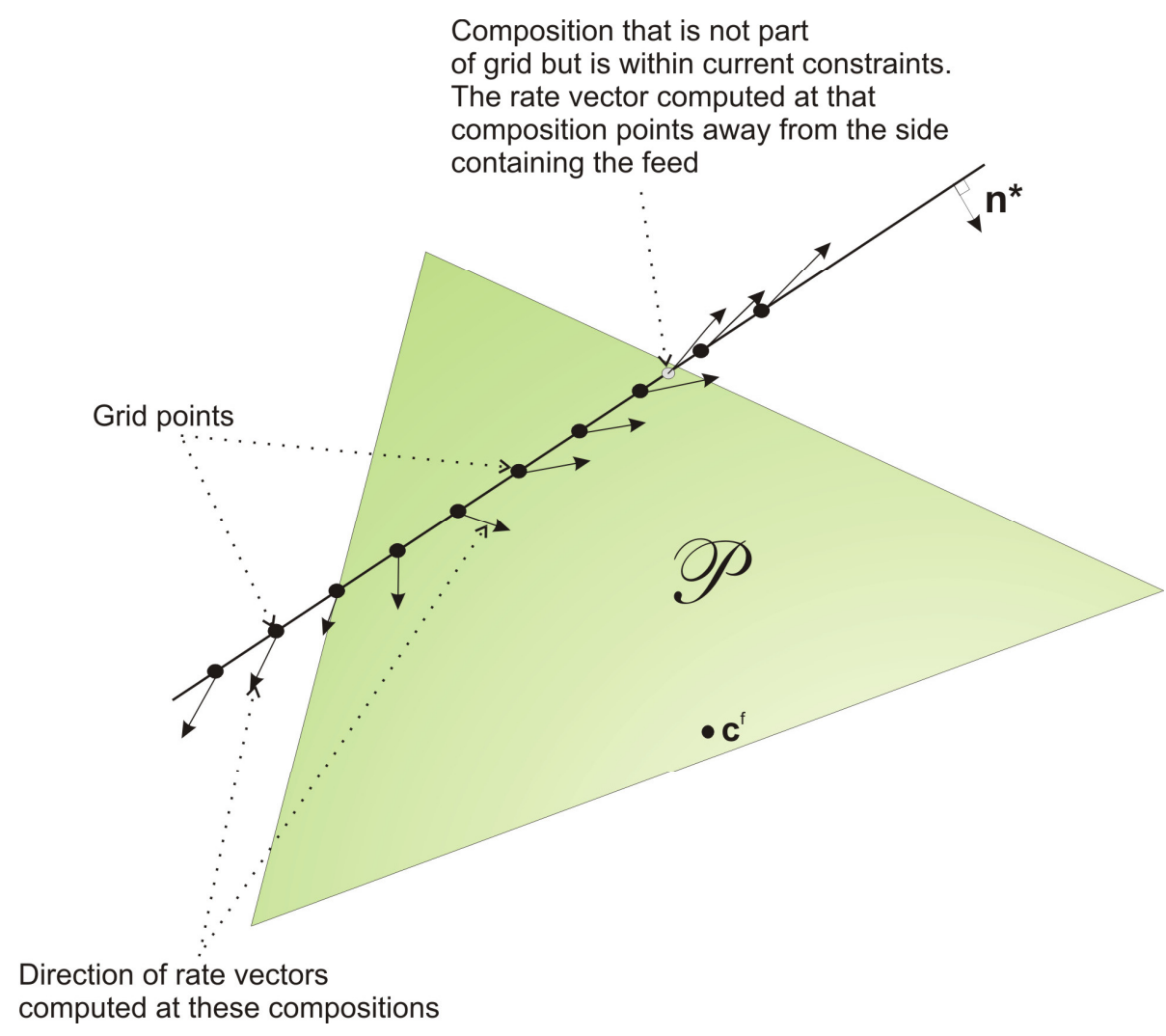

Figure 6.9 Example showing how discretization could "miss" compositions of interest

the other invalid(as shown in the figure), whose rate vector points away from the side containing the feed forcing us to retreat back (based on the procedure described) and not accept the current position of the hyperplane. Since our discretization in this example missed this interesting composition, we have made a hyperplane movement that was not truly valid.

The magnitude of the hyperplane step size was another source of error during the computation. Since the hyperplane offset was being increased by discrete values, compositions where the rate vectors pointed away from the side containing the feed would be missed by the user-created grid. Figure 6.10 shows an intermediate computation where we have two hyperplanes, one with offset $\gamma^{*}+\delta$ and the other 


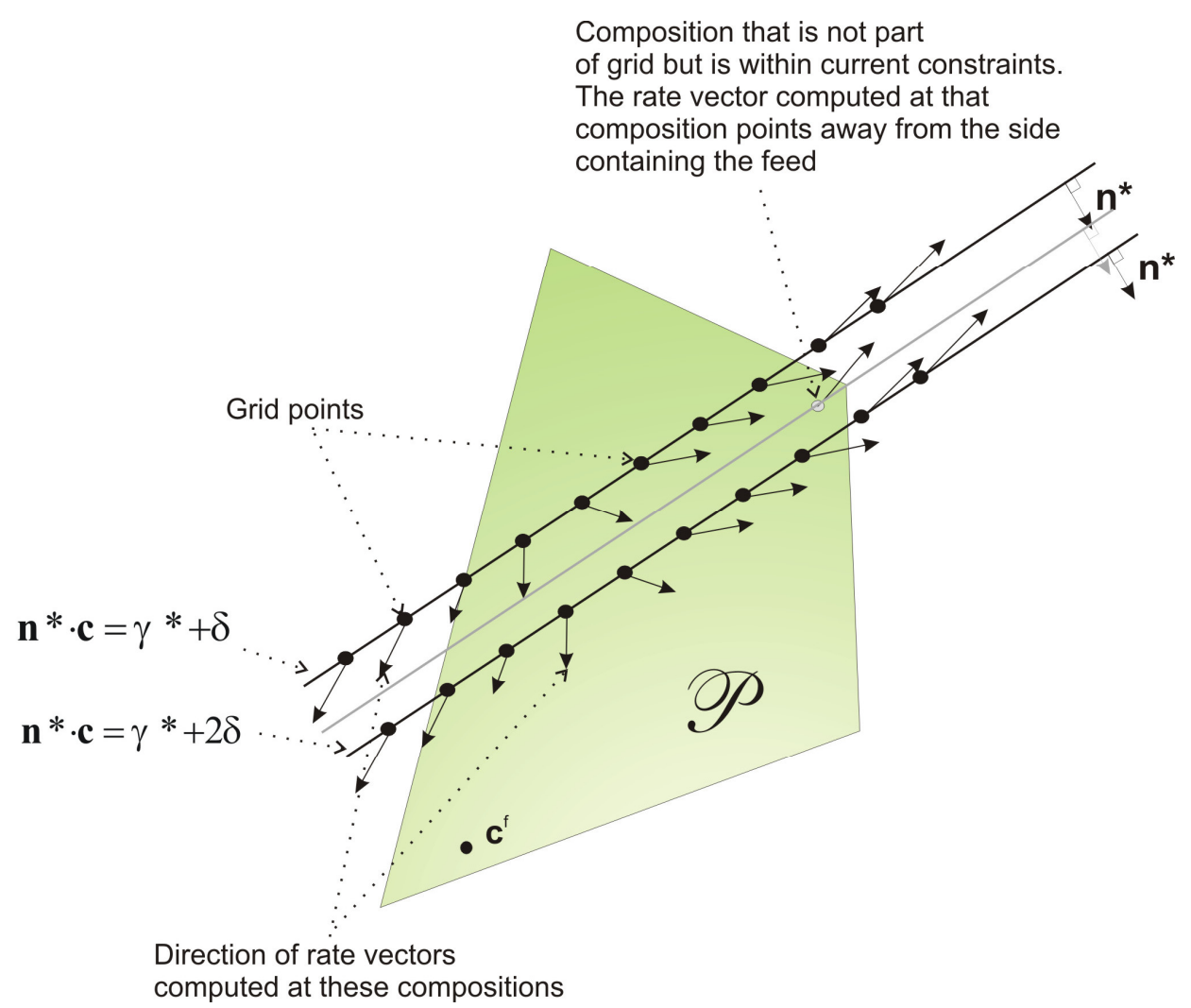

Figure 6.10 Example showing how the hyperplane step size might cause inadvertent skipping of interesting compositions

$\gamma^{*}+2 \delta$ moving inside a current polytope. Note that the valid grid points on both the hyperplanes point to the side containing the feed. However, between the two hyperplanes there exists a composition (inside the current polytope) whose rate vector points away from the side containing the feed if the hyperplane with normal $\mathbf{n} *$ was passing through it. The algorithmic gridding and moving of the hyperplane did not "catch" this composition which would have prevented the invalid increase of the offset from $\gamma^{*}+\delta$ to $\gamma^{*}+2 \delta$. 
The drawbacks encountered in Maple served as a guide in searching for a better tool and improved techniques in implementing the method of bounding hyperplanes. The next section deals with a vastly improved implementation using a nonlinear optimization tool called SolverDLL and the techniques used were mostly motivated from the Maple implementation while dealing with the drawbacks through "smarter" algorithms.

\subsection{Implementation using "Solver DLL"}

The Solver DLL based implementation of the method of bounding hyperplanes was motivated by some of the drawbacks encountered while trying to use Maple as described in Section 6.2. The SolverDLL method also has the advantage that the instructions given to the computer is through a lower-level language(like $\mathrm{C}++)$ and hence the interpretation of the instructions is faster than through an interface like Maple, which has several layers of instruction. The final package developed runs independently without the need to run other software simultaneously.

There are several software components that were combined to create the SolverDLL-based implementation. Solver DLL is a software tool, distributed by Frontline Systems, Inc., that enables the user to access various optimization engines created by the company. The optimization engines have the ability to solve different optimization problems like linear, nonlinear, mixed-integer and so on. Our interest will be in the nonlinear optimization engine alone (this will be discussed in further detail in the next section). 
Though the core of the software implementation is Solver DLL, other components are important in implementing the method in a fast and accurate manner. We use the source code of cdd (already described earlier) available in the form of $c d d l i b$ [91] for computation of extreme points from a given set of halfspaces. Another important part of the software is GMP (GNU Multiple Precision Arithmetic Library) [92] which performs extremely accurate arithmetic operations and is used in conjunction with $c d d l i b$. The algorithm used in the software can be described by using four modules each performing certain tasks required in the method. These modules are called the Main, Solver, Cdd and Auxiliary modules. While the Main, Solver and Auxiliary modules were written in Object Pascal, the Cdd module was written in the $\mathrm{C}++$ computer language. The "Borland $\mathrm{C}++$ Builder" software package allows easy integration of the modules written in different programming languages, but the specifics about programming structure will not be discussed. Figure 6.11 shows broadly how the program functions and how control moves in and out of the four modules.

The Main module controls the progress of the overall computation and gives the user access to the other modules. It also contains conditions that let the program decide what modules to use and when they are to be used. The first task performed in the Main module is to obtain the current constraints on the set of achievable compositions (i.e. the current polytope). 


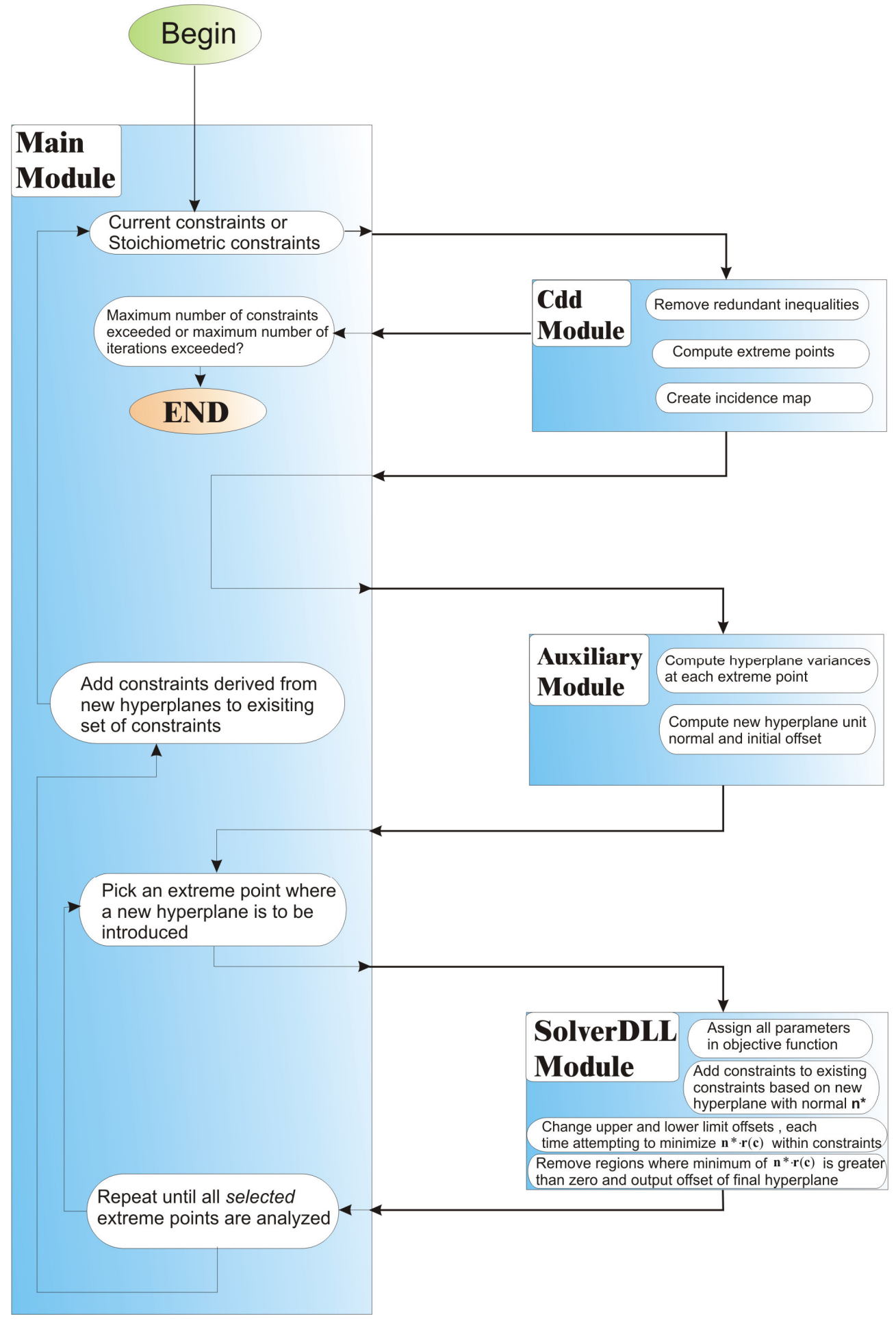

Figure 6.11 Program structure for the Solver DLL implementation of the method of bounding hyperplanes 
We can compute the stoichiometric constraints for the system, based on the feed stream composition, externally using software like Maple. This entry point for the program, alternatively, permits loading of constraints computed in a previous session into the module.

The next step is to find the extreme points for the given set of constraints and to create an incidence map both using the Cdd module. The inequalities from the Main module are received as an array of data in a standard format. There are three main tasks performed inside this module. The first task is the removal of redundant inequalities using the library functions of $c d d l i b$. This involves some simple linear programming algorithms that are explained in detail in the appendix. The second task is the computing of extreme points using the nonredundant inequalities. Once this is complete, the final task involves creating an incidence map that describes which inequality is incident at each extreme point. After performing these tasks, control returns to the Main module.

In the course of executing the above tasks, the Cdd module accesses high precision arithmetic tools available in GMP. The most important of these high precision tools gives the ability to do computation where operands are in the form of rational numbers. Without GMP, it was found that the libraries in cddlib often gave erroneous results especially when the computation involved several inequalities that had similar normals and offsets. Use of GMP, and especially the rational number-based arithmetic tools, gave consistently accurate results. There is, however, a penalty paid in terms of computation time by using GMP and one would need to decide, based on the problem, if the tradeoff between accuracy and computation time is warranted. In all our 
computations, GMP with rational numbers was used. Further details about GMP and rational number arithmetic can be seen in the appendix.

The Main module, after receiving computational output from the Cdd module, then moves program control into the Auxiliary module. In the Auxiliary module, several computations are made that are meant to efficiently and "smartly" create and move hyperplanes in the Main module.

A part of the code in the Auxiliary module evaluates the sharpness or smoothness at every extreme point and computes the normals for a new hyperplane that could be introduced at each of these points. The input to this sub-module receives an array of extreme points, nonredundant inequalities and the incidence map. The output of the sub-module returns an array of hyperplane variances computed at each extreme point and a median value is ascertained. This is because we want to focus our computational efforts in creating hyperplanes where the extreme points are sharper. The reason for this is that theory describing general properties of the attainable region suggests that the boundary of the attainable region is made up of smooth surfaces and straight-line sections [4]. By successively "cutting away" sharper extreme points with hyperplanes, the goal is to get a smooth surface that might tightly bound compositions that are attainable.

The Auxiliary module also computes the normals for a new hyperplane that might be introduced at the extreme point (as required by the method). The computations described below are only performed for those extreme points which have been selected by the user based on the hyperplane variances. We use the same idea described in the previous section that any new hyperplane must have a normal that is a convex combination of the normals of the hyperplanes incident at that extreme point. If 
$\mathbf{n}^{*}$ is the unit normal perpendicular to the new hyperplane and if $\mathbf{n}_{1}, \mathbf{n}_{2}, \ldots, \mathbf{n}_{k}$ are normals representing the hyperplanes incident at the extreme point, then

$$
\begin{aligned}
& \mathbf{n}^{*}:=\sum_{i=1}^{k} \lambda_{i} \mathbf{n}_{i}, \\
& 0<\lambda_{i}<1 .
\end{aligned}
$$

Here $\lambda_{i}$ is a weighting factor for each hyperplane and two weighting factors were made available in the Auxiliary module:

i) Equal Weight factor: This option computes the new hyperplane by giving equal weight to each normal for the hyperplanes incident at the extreme point. In other words, $\lambda_{i}:=1 / k$ for each value of $i$

ii) Biased Weight factor : We define a biased weight factor by giving emphasis to those normals that deviate further away from the normalized average :

$$
\lambda_{i}:=\frac{1}{k \sigma_{j}^{p}}\left\|\mathbf{n}_{i}-\overline{\mathbf{n}}\right\|^{p},
$$

where $\sigma_{j}^{p}$ is a modified hyperplane variance at the $j$ th extreme point defined as

$$
\sigma_{j}^{p}:=\frac{1}{k} \sum_{i=1}^{k}\left\|\mathbf{n}_{i}-\overline{\mathbf{n}}\right\|^{p},
$$

and $\overline{\mathbf{n}}$ is the normalized average of hyperplane normals incident at the extreme point. The parameter $p$ is the power parameter and determines how much bias should be given to a normal deviating from the normalized average $\overline{\mathbf{n}}$. If the power parameter is set to 2 , then more bias will be given to the normals that deviate to a large extent from $\overline{\mathbf{n}}$ than if the parameter value is set to 1 . Using the biased weight factor, the new hyperplane normal, $\mathbf{n}$ *, can be computed by the following relations: 


$$
\begin{gathered}
\mathbf{n}_{a v g}:=\frac{1}{k \sigma_{j}^{p}} \sum_{i=1}^{k}\left\|\mathbf{n}_{i}-\overline{\mathbf{n}}\right\|^{p} \mathbf{n}_{i}, \text { and } \\
\mathbf{n}^{*}:=\frac{\mathbf{n}_{\text {avg }}}{\left\|\mathbf{n}_{\text {avg }}\right\|} .
\end{gathered}
$$

The power parameter is a user-controlled parameter and we found that a value of 1 created hyperplanes that trimmed larger portions of infeasible composition space when compared to results obtained while setting a value of 2 .

Once the normal for the new hyperplane is computed, its offset is also computed such that the hyperplane coincides with the extreme point. If $\mathbf{c} *$ is an extreme point and $\mathbf{n} *$ is the normal of a new hyperplane coinciding with it, then the equation of the hyperplane can be calculated from $\gamma^{*}=\mathbf{n} * \cdot \mathbf{c} *$. This step is the final computation in the Auxiliary module, and control returns to the Main module.

In the Main module, the next computation involves the introduction and movement of new hyperplanes. The user has the option of either examining all extreme points or examining selected extreme points based on the hyperplane variances described earlier. Our experience with being selective in the choice of extreme points being examined has been mixed. On one hand, examining all extreme points is computationally intensive, and probably only a few new hyperplanes might be introduced. The inability to add bound trimming hyperplanes (even when the software creates hyperplanes and tries to move them) is mostly due to hurdles in implementation rather than a drawback of the method. To optimize computational resources and to minimize computation time we fix the maximum number of iterations and the minimum required change in offset when moving a hyperplane. Such limits might prevent the addition of new hyperplanes that 
have very small change in offset or need a very large number of iterations to compute the desired offset. However, the bounds obtained were found to be tighter than those obtained by being selective.

Examining extreme points selectively was found to be inefficient when there were only a few extreme points (for example, when extreme points arise from less than 100 nonredundant hyperplanes for a system with 4 species) and efficient when there were a large number of extreme points (for example, when the extreme points arise from more than 500 nonredundant hyperplanes for a system with 4 species). Our final implementation utilizes one of the two options in alternate iterations, i.e. in one iteration all extreme points are examined while in the consecutive iteration only selected extreme points are examined. This was done because for a general problem it was hard to know prior to computations what the reasonable number of extreme points were that would benefit from selective examining of extreme points.

At each extreme point, the first check that is made is whether it coincides with the feed stream composition. It does not make sense to try to move a hyperplane that is already incident with the feed stream since it will automatically violate one of the two conditions that we seek. If there is more than one feed stream and if an extreme point coincides with any one of them, then such an extreme point can also be ignored for the purposes of introducing a new hyperplane.

The next step involves using the hyperplane equation obtained from the Auxiliary module by using the biased weight factor method described earlier. The program control then moves to the Solver module where computations aimed at moving the hyperplane are executed. 
In the next few paragraphs, we will discuss the working of the Solver module including input to, and output from the module. The module receives as input from the Main module, data corresponding to the set of constraints describing the current polytope. It also receives data describing the equation for the new hyperplane that is to be moved along with a set of parameters needed for the optimization engine in the Solver module.

The Solver module accesses Solver DLL functions that perform nonlinear optimization (a more general description on the functionality of the Solver DLL is given in the appendix). The optimization function generally solves for a system of $n$ variables by either maximizing or minimizing a nonlinear function consisting of these $n$ variables subject to constraints on the variables. Let $\mathbf{x} \in \mathbb{R}^{n}$ where $\mathbf{x}:=\left[x_{1}, x_{2}, \ldots . ., x_{n}\right]$, and $g(\mathbf{x})$ be any scalar-valued function of $\mathbf{x}$, either linear or nonlinear in the components of $\mathbf{x}$. that will be minimized or maximized subject to the constraints

$$
h_{1}(\mathbf{x}) \leq b_{1}, h_{2}(\mathbf{x}) \leq b_{2}, \ldots \ldots . ., h_{\mathrm{q}}(\mathbf{x}) \leq b_{q},
$$

where $h_{i}(\mathbf{x})$ is any scalar function of $\mathbf{x}$, and $b_{i}$ is any real valued number, which together constitute the $i^{\text {th }}$ constraint from among a list of $q$ constraints. For our computations, $h_{i}(\mathbf{x})$ will mostly be a linear function in the components of $\mathbf{x}$.

The core of the Solver module implements the method of bounding hyperplanes by using nonlinear optimization. Figure 6.12 and Figure 6.13 will assist in describing the steps involved in the algorithm used. 


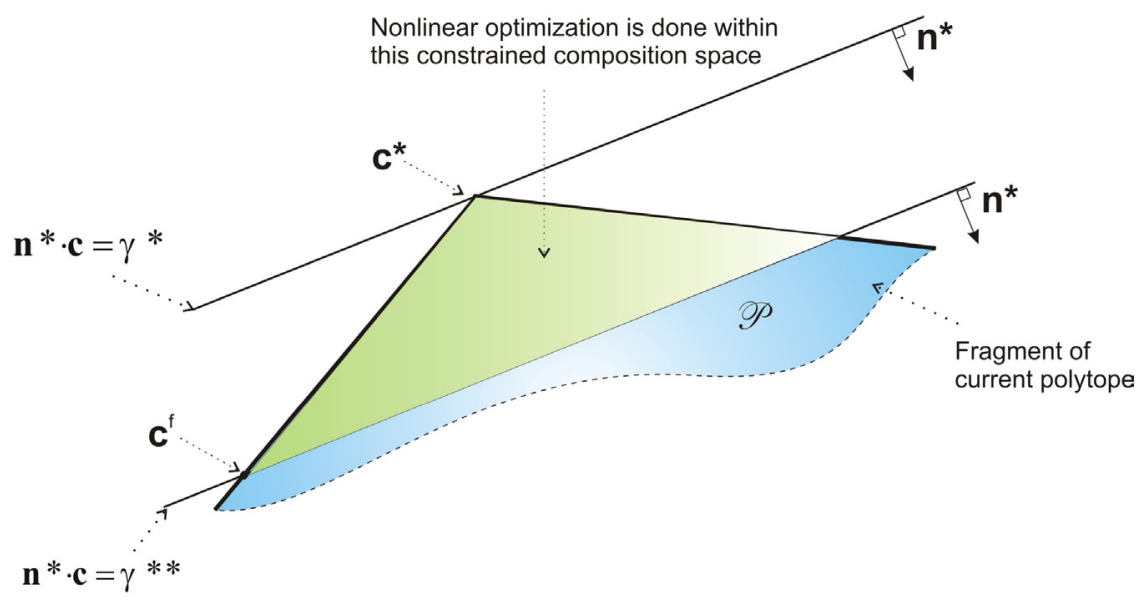

Figure 6.12 Figure showing lower limit and upper limit added to the current polytope as part of initialization in the Solver DLL module

We begin with the current polytope, $\mathscr{P}$, and at an extreme point introduce a new hyperplane, say with normal $\mathbf{n}$, and move the hyperplane into the current polytope. The movement of the hyperplane into the current polytope is achieved through an algorithm involving iterative calls to the Solver DLL program.

Figure 6.12 shows the initialization done in the Solver DLL module. A new hyperplane with a unit normal $\mathbf{n} *$ is introduced into the current polytope. The lower limit hyperplane, $\mathbf{n} * \cdot \mathbf{c}=\gamma^{*}$ passes through the extreme point currently being examined and the upper limit hyperplane, $\mathbf{n} * \cdot \mathbf{c}=\gamma^{* *}$ passes through the feed stream composition $\mathbf{c}^{\mathrm{f}}$.(Though the lower and upper limit hyperplanes are initially chosen, they are changed dynamically as the iterations progress.) We now turn our focus to the region between the lower limit and the upper limit and the constraints that correspond to that region can be written as

$$
\begin{aligned}
& \mathbf{n} * \mathbf{c} \geq \gamma^{*} \\
& \mathbf{n}^{*} \cdot \mathbf{c} \leq \gamma^{* *} .
\end{aligned}
$$



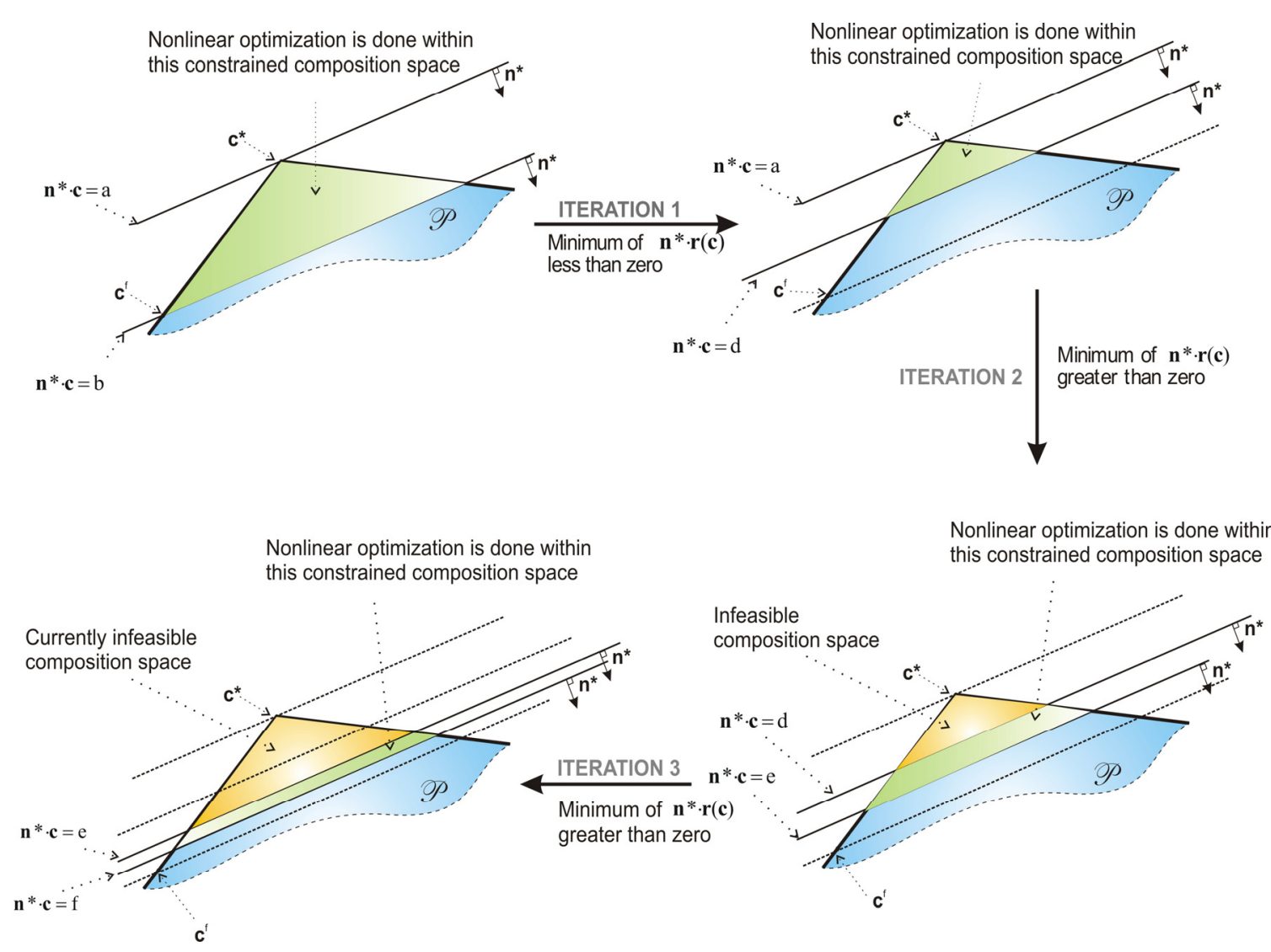

Figure 6.13 Iterations in the Solver module showing constraints being moved such that the optimization indicates those regions that are infeasible

The composition set formed by the combination of the current polytope (obtained from the Main module) and the two new constraints (equation 6.30) will define the variable space within which Solver DLL does nonlinear optimization. In Figure 6.13, this is the region shown in green.

After defining the constraints for optimization completely, the next step is to define the objective function. Based on the method of bounding hyperplanes, we define the objective function for the optimization such that we would like to find out what the minimum of $\mathbf{n} * \cdot \mathbf{r}(\mathbf{c})$ is within the constrained space defined. If 
$\mathbf{n}^{*}:=\left[n_{1}^{*}, n_{2}^{*}, \ldots . ., n_{N}^{*}\right]$ and $\mathbf{r}(\mathbf{c}):=\left[r_{1}(\mathbf{c}), r_{2}(\mathbf{c}), \ldots \ldots, r_{N}(\mathbf{c})\right]$, then the objective function to be minimized might be written as

$$
n_{1}^{*} r_{1}(\mathbf{c})+n_{2}^{*} r_{2}(\mathbf{c})+\ldots . .+n_{N}^{*} r_{N}(\mathbf{c})
$$

If the minimum of the function in equation (6.31) is found to be less than zero this means that there is some (at least one) composition within the constraints selected where $\mathbf{n} * \mathbf{r}(\mathbf{c})<0$. This means we cannot discard the region within the current constrained composition space based on the method of bounding hyperplanes. The next iteration would then involve trying to reduce the offset to find hyperplanes that constrain compositions such that $\mathbf{n} * \mathbf{r}(\mathbf{c})>0$.

If the minimum of the objective function is found to be greater than zero then we know that all compositions within the current constrained space satisfy the relation $\mathbf{n} * \cdot \mathbf{r}(\mathbf{c})>0$. This means we can discard compositions described by the chosen constraints and try to push the upper limit hyperplane forward even more. If the upper limit hyperplane is already one that passes through the feed composition then no further movement of the hyperplanes is permitted.

When the minimum of the objective function is found to be equal to zero then we deal with it in a slightly different manner which will be explained after an illustration to demonstrate how we deal with the first two situations.

Figure 6.13 shows a series of iterations in the Solver module meant to move a new hyperplane into the current set of feasible compositions and attempt to remove regions that are deemed infeasible by the method of bounding hyperplanes. Hyperplanes with normal $\mathbf{n} *$ are first introduced with two distinct offsets. One 
hyperplane passes through the extreme point being examined in the Main module while the other passes through the feed stream composition. Their equations are $\mathbf{n} * \mathbf{c}=\mathbf{a}$ and $\mathbf{n} * \mathbf{c}=\mathrm{b}$ where the first is the lower limit and the latter is the upper limit. The corresponding half spaces added to existing ones would be $\mathbf{n} * \mathbf{c} \geq \mathrm{a}$ and $\mathbf{n} * \mathbf{c} \leq \mathrm{b}$. The Solver DLL engines then computes the minimum of $\mathbf{n} * \mathbf{r}(\mathbf{c})$ within the constraints and in the example shown in the figure the value turns out to be less than zero. After this first iteration, we keep the lower limit unchanged while changing the upper limit to the new hyperplane $\mathbf{n} * \mathbf{c}=d$ where the offset $d$ is simply $(a+b) / 2$. Again the minimum of $\mathbf{n}^{*} \cdot \mathbf{r}(\mathbf{c})$ within the constraints is computed and found to be greater than zero. According to the theory, then, all compositions within the current constraints are not compositions that are attainable through reaction and mixing alone (for the given feed.)

After the second iteration, we change the lower limit and the upper limit based on the optimization result. The hyperplane $\mathbf{n} * \mathbf{c}=\mathrm{d}$ is the new lower limit and hyperplane $\mathbf{n} * \mathbf{c}=\mathrm{e}$ is the new upper limit (where e: $=(\mathrm{d}+\mathrm{b}) / 2$.) In the third example iteration, the minimum of $\mathbf{n} * \mathbf{r}(\mathbf{c})$ within the constraints is greater than zero; hence the region between current constraints and the hyperplanes with offsets $d$ and e can be considered a region with infeasible compositions. Subsequently, a new offset, f, is computed as before and iterations proceed. The upper limit hyperplane represents the furthest a hyperplane can be pushed into the current polytope.

If the minimum of the objective function is found to be equal to zero, this means that $\mathbf{n} * \mathbf{r}(\mathbf{c})=\mathbf{0}$ for some composition within the chosen hyperplanes. Assuming that $\mathbf{n}^{*} \neq \mathbf{0}$ we can have either $\mathbf{r}\left(\mathbf{c}^{\prime}\right)=\mathbf{0}$ or $\mathbf{r}\left(\mathbf{c}^{\prime}\right) \neq \mathbf{0}$ where $\mathbf{c}^{\prime}$ is the composition at 
which the objective function evaluates to zero. If $\mathbf{r}\left(\mathbf{c}^{\prime}\right)=\mathbf{0}$ then $\mathbf{c}^{\prime}$ is an equilibrium composition and in this case the upper limit hyperplane can be pushed further into the current polytope. If $\mathbf{r}\left(\mathbf{c}^{\prime}\right) \neq \mathbf{0}$ then $\mathbf{c}^{\prime}$ is not an equilibrium composition and in this case we cannot push the upper limit hyperplane any further.

Once the minimum of the objective function is determined, we follow the described algorithm of changing the offsets for the lower limit and upper limit until we are able to eliminate a large portion of composition space in which all (nonequilibrium) compositions satisfy the inequality $\mathbf{n} * \mathbf{r}(\mathbf{c})>0$. To control computational load, the movement of offsets is controlled by various parameters: (1) Number of Solver DLL iterations, where each iteration involves changing either or both of the upper and lower limit offsets and then executing a nonlinear optimization function; (2) Objective cutoff value: This is the smallest (positive) permissible value for the objective function (eqn 6.31) that we permit for regions that can be removed. If the objective cutoff is set to be a value like $10^{-2}$, the iterations (and hence the computational load) would be less as compared to a smaller cutoff value like $10^{-6}$. (3) Absolute difference between the offsets of the upper and lower limit hyperplanes: a small difference in the offset means only a small region of composition space has the probability of being removed. In certain situations, it makes sense to focus on those regions where the offset difference is large. The absolute difference between the offsets of the upper and lower limit hyperplanes was permitted a minimum value of $10^{-6}$ to obtain tighter bounds while a minimum value of $10^{-4}$ was sufficient to give coarsely approximate bounds. 
Once the hyperplane is moved to the furthest possible position we can remove this region to give a smaller bounding set. This is also referred to as removal of kinetically infeasible composition space.

The Solver module contains a few other user-controlled parameters but these are specific to Solver DLL and cannot be used in all nonlinear optimization software. Therefore, these parameters will not be discussed here but a brief overview is given in the appendix.

The output from the Solver module gives the offset value associated with the hyperplane that is found using the computations mentioned above. It may so happen that the offset returned is the same as the initial offset computed for the hyperplane passing through the selected extreme point. If this occurs then the new hyperplane is ignored and not added to the list of new hyperplanes. We can also ignore the new hyperplane if the computed offset is very close to the initial offset. In other words, we can ignore the new hyperplane if the absolute difference between the initial offset and the computed offset is less than a small finite value like $10^{-6}$. If the new hyperplane is not added to the list of new hyperplanes then another attempt is made using a hyperplane of different orientation (i.e. different unit normal) at the same extreme point. Typically we try moving a hyperplane generated by the biased-weight-factor first, followed by a hyperplane created using the equal-weight-factor (both available in the Auxiliary module)

After examining all extreme points, the Main module combines the existing constraints with the new hyperplanes (through the associated halfspaces). The complete list of inequalities is given as an input to the Cdd module which computes a list of nonredundant inequalities and the extreme points of a new bounding set (i.e. the 
current polytope for the next iteration loop). The Main module then repeats the entire process of selecting extreme points, introducing new hyperplanes and moving the hyperplanes as outlined earlier. The entire sequence is an iteration in the Main module. We can set the maximum number of iterations in the main module to control the entire program execution. For most of the examples worked out in Chapter 7 of this thesis, the maximum number of iterations in the main module was limited by the number of

constraints permitted in the nonlinear optimization software. Two optimization engines of Solver DLL were used (explained in the appendix) in the program. The basic engine called "small-scale GRG" has a limit of 250 constraints while an advanced engine called "LGO" has a limit of 1000 constraints.

\subsection{Limitations of the Solver DLL implementation}

There were some difficulties encountered in the Solver DLL method and these can be classified as either software-based or algorithm-based limitations.

The software-based limitations were mostly due to the fact that we were dependent on externally procured nonlinear optimization software, Solver DLL, that is tailored to solve single optimizations. Our technique requires solution of a sequence of optimization problems, each with new constraints and sometimes with a change in the objective function.

Solver DLL is supplied with several parameters that need to be tuned depending on the optimization problem solved. For a given optimization problem, this tuning of parameters is generally done in an ad hoc manner and is mostly based on trial 
and error. Once a set of well-tuned parameters has been found for a single problem, Solver DLL (and, in general, most nonlinear optimization software) will easily solve that optimization problem. Given the fact that our implementation of the method of bounding hyperplanes in Solver DLL uses varying constraints and objective functions, finding a set of well-tuned parameters is difficult.

A nonlinear optimization problem has two major components, the constraints and the objective function. In our case, the constraints are always linear in nature whereas the objective functions tend to be mostly nonlinear. Hence for every reaction network, the Solver DLL might have a different objective function that needs a different set of optimization parameters. Even with a fixed reaction network, we have to deal with a new objective function for each new choice of a hyperplane normal $\mathbf{n}$. The stress on finding the right set of parameters for a nonlinear optimization problem is to avoid the common pitfall of such software that often determine a local optimum rather than the global optimum .

For a given optimization problem, the objective function can have several local optima but only a single global optimum. An example for local and global optima is shown in Figure 6.14 which is a plot of a variable $\mathrm{x}$ against a (nonlinear) function of $\mathrm{x}$, $\mathrm{f}(\mathrm{x})$. If the objective of the optimization is to minimize $\mathrm{f}(\mathrm{x})$, then $\mathrm{x}_{1}, \mathrm{x}_{2}$ and $\mathrm{x}_{3}$ are local optima whereas $\mathrm{x}_{3}$ is the single global optimum for all values of $\mathrm{x}$ (within the plot range.) In our program, the Solver module attempts to find global optima while trying to find the minimum of $\mathbf{n} * \cdot \mathbf{r}(\mathbf{c})$ (for all vectors $\mathbf{c}$ within defined constraints), but if a poor set of parameters is chosen the Solver DLL may compute a local optimum instead. 


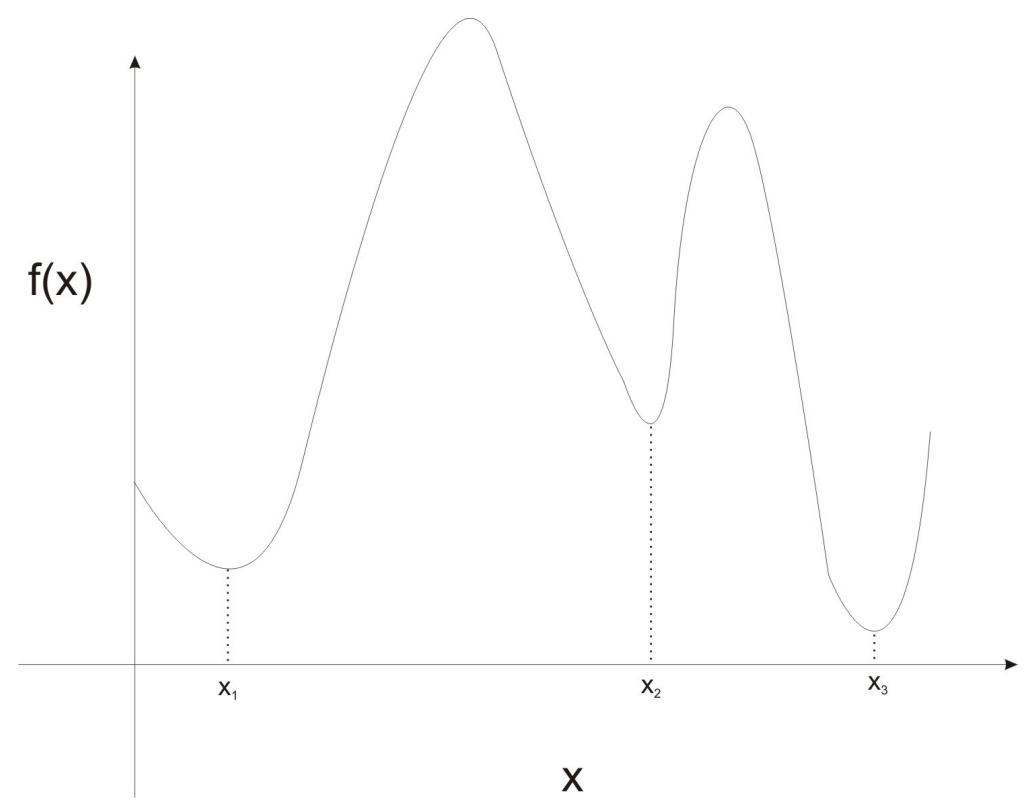

Figure 6.14 A simple function plot showing several local optima (and a single global optimum)

Algorithmic-based issues were faced in attempting to decide (1) how to pick extreme points to be examined (2) how to choose an orientation for the new hyperplane introduced at an extreme point and (3) how to pick the right offset when moving the hyperplanes so that kinetically infeasible regions can be removed with fewer iterations? These issues will be discussed in Chapter 8 after we look at their effect in the examples presented in Chapter 7. 


\section{CHAPTER 7}

\section{CASE STUDIES}

In this chapter, the goal is to look at how the method of bounding hyperplanes can be applied to find kinetic bounds on the attainable region for the pure reactor synthesis problem. We will present a few results obtained using the Maple platform and more complex examples studied using the Solver DLL implementation. We will compare the bounds computed by the program with effluent stream compositions from typical reactor configurations. The case studies will also reiterate the fact that our method is meant to complement existing methods and not to replace them.

All the examples presented in this chapter will deal with systems permitting reactions and mixing alone. They are incompressible single-phase systems that are homogeneous and are operated under isothermal conditions. In each of the case studies, we are trying to answer the following question: For a system permitting reaction and mixing alone, what is the full set of achievable compositions for the given reaction network and the prescribed feed stream. Rather than describe the actual compositions that answer this question, our results prescribe outer bounds within which such achievable compositions are assured to lie. 
Since we do not know the actual boundary for the pure reactor synthesis attainable region, the task of evaluating our results becomes difficult. There is, however, theory that describes what reactor configurations might reside on the boundary of the attainable region. Using this idea, we compare the bounds constructed with the output compositions obtained by solving design equations involving various reactor configurations. We refer to these computed compositions as a set of achievable compositions.

\subsection{Preliminaries}

Part of the following material is repeated from Chapter 6 as a review prior to introducing the concept of projecting convex hulls.

Let $V$ be a real finite-dimensional vector space that contain the set of vectors $\left\{\mathbf{x}_{1}, \mathbf{x}_{2}, \ldots \ldots, \mathbf{x}_{k}\right\}$. We can say that a vector $\mathbf{v}$ in $V$ is a convex combination of $\left\{\mathbf{x}_{1}, \mathbf{x}_{2}, \ldots \ldots ., \mathbf{x}_{k}\right\}$ if there exists a set of numbers $\left\{\lambda_{1}, \lambda_{2}, \ldots \ldots ., \lambda_{k}\right\}$ such that

$$
\begin{gathered}
\lambda_{i} \geq 0, \quad i=1,2, \ldots ., k, \\
\sum_{i=1}^{k} \lambda_{i}=1,
\end{gathered}
$$

and

$$
\mathbf{v}=\sum_{i=1}^{k} \lambda_{i} \mathbf{x}_{i}
$$

If $\mathbf{v}$ and $\mathbf{w}$ are distinct vectors in $V$, then a line segment that connects $\mathbf{v}$ and w is the set of all vectors in $V$ that reside in the set given by 


$$
\{\lambda \mathbf{v}+(1-\lambda) \mathbf{w} ; 0 \leq \lambda \leq 1\}
$$

In other words, the line segment that connects $\mathbf{v}$ and $\mathbf{w}$ is the set of all convex combinations of $\mathbf{v}$ and $\mathbf{w}$. The interior of the line segment refers to the set of points corresponding to $0<\lambda<1$.

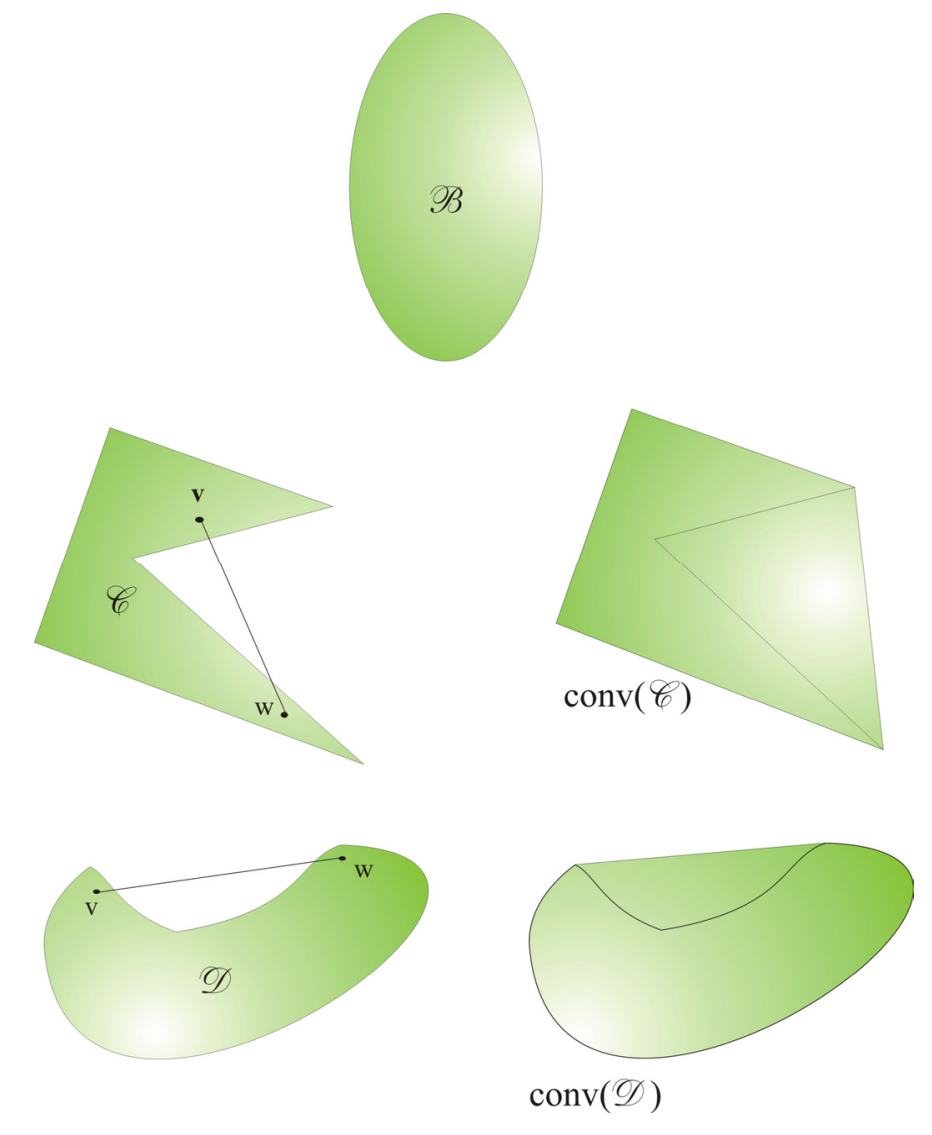

Figure 7.1 Examples for a convex set, non-convex set and convex hull

Let $\mathcal{A}$ be a set of vectors in a vector space $V$. Such a set is called convex if for all vectors $\mathbf{v}$ and $\mathbf{w}$ that are in $\mathscr{A}$, the set $\mathscr{A}$ contains the complete line segment connecting $\mathbf{v}$ and $\mathbf{w}$. The convex hull of $\mathscr{A}$, denoted by conv( $\mathscr{A})$, refers to the smallest convex set in $V$ that contains $\mathscr{A}$ (In other words, $\operatorname{conv}(\mathscr{A})$ is the intersection of all 
convex sets that contain $\mathscr{C})$. The set $\operatorname{conv}(\mathscr{C})$ may also be defined as the set of all convex combinations of finite sets of vectors taken from $\mathscr{A}$. If $\mathscr{A}$ is a convex set then $\operatorname{conv}(\mathscr{A})$ is identical to $\mathscr{A}$.

In Figure $7.1, \mathscr{B}$ is a convex set while $\mathscr{C}$ and $\mathscr{D}$ are not convex sets since part of the line segment connecting the vectors $\mathbf{v}$ and $\mathbf{w}$ lie outside the set. The convex hull of $\mathscr{C}$ and $\mathscr{D}$ are also shown in Figure 7.1.

Let $\left\{\mathbf{v}_{1}, \mathbf{v}_{2}, \ldots . ., \mathbf{v}_{\mathrm{k}}\right\}$ be the vertices of a convex polytope $\mathscr{A}$ (see definition of convex polytopes in Chapter 5 Section 5.1) in a vector space $V$. In other words, $\mathbf{v}_{1}, \mathbf{v}_{2}, \ldots . ., \mathbf{v}_{\mathrm{k}}$ are the extreme points of $\mathscr{C}$ and $\mathscr{A}=\operatorname{conv}\left(\mathbf{v}_{1}, \mathbf{v}_{2}, \ldots ., \mathbf{v}_{\mathrm{k}}\right)$. If $T: V \rightarrow W$ is a linear transformation from $V$ to a vector space $W$ (which might have a dimension less than or equal to $W$ ) then

$$
T(\mathscr{A})=T\left(\operatorname{conv}\left(\mathbf{v}_{1}, \mathbf{v}_{2}, \ldots ., \mathbf{v}_{\mathrm{k}}\right)\right)=\operatorname{conv}\left(T \mathbf{v}_{1}, T \mathbf{v}_{2}, \ldots ., T \mathbf{v}_{\mathrm{k}}\right)
$$

The purpose of deriving equation (7.1) is to show the general validity of projecting convex sets from higher dimensions to lower dimensions as will be done in the presentation of results in this chapter.

When dealing with problems having a large number of species (greater than three), it is not possible to visualize the results showing the concentrations of all the species simultaneously. Therefore we choose two or at most three species whose concentration bounds we would like to visualize. In such a case, it becomes necessary to project the computational results from a higher dimension to a lower dimension.

For example, if we have a problem with six species then our computational output will have extreme points in a six-dimensional space (and hence 
cannot be visualized) that represent a set of feasible compositions, $\mathscr{P}$. We will have extreme points of the form $\left[c_{1}, c_{2}, c_{3}, c_{4}, c_{5}, c_{6}\right]$ where $c_{i}$ is the concentration of species $\mathrm{A}_{\mathrm{i}}$. Suppose we are interested in looking at the kinetic bounds on attainable concentrations of species $\mathrm{A}_{2}$ and $\mathrm{A}_{6}$. Then we will look at $\left[c_{2}, c_{6}\right]$ pairs and in particular those pairs that are extreme points of the current polytope. We can interpret this step as a linear transformation from a vector space of six dimensions to one of two dimensions. That is, in this example, $T$ is a linear transformation that maps $\mathbb{R}^{6}$ to $\mathbb{R}^{2}$ according to the rule $T\left[X_{1}, X_{2}, X_{3}, X_{4}, X_{5}, X_{6}\right]=\left[X_{2}, X_{6}\right] . T$ takes $\mathscr{P}$ into its projection onto $\mathbb{R}^{2}$. From equation (7.1) we learn that such a transformation is achieved by first mapping each of the extreme points and then finding its convex hull. In other words, we take each extreme point from the output of the computations (that also reside in $\mathscr{P}$ ) and pick the $2^{\text {nd }}$ and $6^{\text {th }}$ component from each of them to make a corresponding $\left[c_{2}, c_{6}\right]$ pair. All such pairs are collected and the convex hull of these pairs is computed to find the extreme points. This projection of convex hulls from high dimensions to lower dimensions will be used to describe kinetic bounds on the concentrations of desired and undesired chemical species in the case studies conducted in this chapter.

\subsection{The Van de Vusse reaction system}

The Van de Vusse reaction (VdV) system is among the most frequently studied reaction network in the literature [3, 60, 93-98]. We will look at variations of the $\mathrm{VdV}$ reaction network and look at how the differences affect our computation and results. 


\subsubsection{The irreversible Van de Vusse Example}

The irreversible Van de Vusse reaction network is described by the following system of reactions along with mass-action rate constants as indicated,

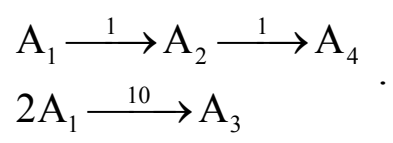

We assume a single feed stream of composition $\mathbf{c}^{\mathrm{f}}=[1,0,0,0]$. Let $c_{1}, c_{2}, c_{3}$ and $c_{4}$ be the concentrations of species $A_{1}, A_{2}, A_{3}$ and $A_{4}$ respectively. The species-formation rate function can be written as follows:

$$
\mathbf{r}(\mathbf{c}):=\left[-c_{1}-20 c_{1}^{2}, c_{1}-c_{2}, 10 c_{1}^{2}, c_{2}\right]
$$

For the given feed stream composition, we can compute the following constraints on concentrations that are stoichiometrically compatible with the feed.

$$
\begin{aligned}
& c_{1} \geq 0 \\
& c_{2} \geq 0 \\
& c_{3} \geq 0 \\
& c_{1}+c_{2}+2 c_{3} \leq 1 \\
& c_{4}=1-c_{1}-c_{2}-2 c_{3}
\end{aligned}
$$

If one is interested in the concentrations of species $\mathrm{A}_{1}$ and $\mathrm{A}_{2}$ alone then we can look at a 2-dimensional plot of $c_{1}$ versus $c_{2}(2-\mathrm{D} \mathrm{VdV})$, whereas if one is interested in studying the concentrations of species $\mathrm{A}_{1}, \mathrm{~A}_{2}$, and $\mathrm{A}_{3}$ simultaneously then we plot $c_{1}, c_{2}$ and $c_{3}$ in a 3dimensional plot (3-D VdV). These two types of cases are studied separately in the following paragraphs. Note that since $c_{4}$ can be computed from the other three 
concentrations, it is adequate to study at most the concentrations of three species since that of the fourth can be computed uniquely.

(A) 2-Dimensional VdV: For the two dimensional VdV plot, we are interested in concentrations of $c_{1}$ versus $c_{2}$ and the stoichiometric constraints are as follows:

$$
\begin{aligned}
& c_{1} \geq 0 \\
& c_{2} \geq 0 \\
& c_{1}+c_{2} \leq 1
\end{aligned}
$$

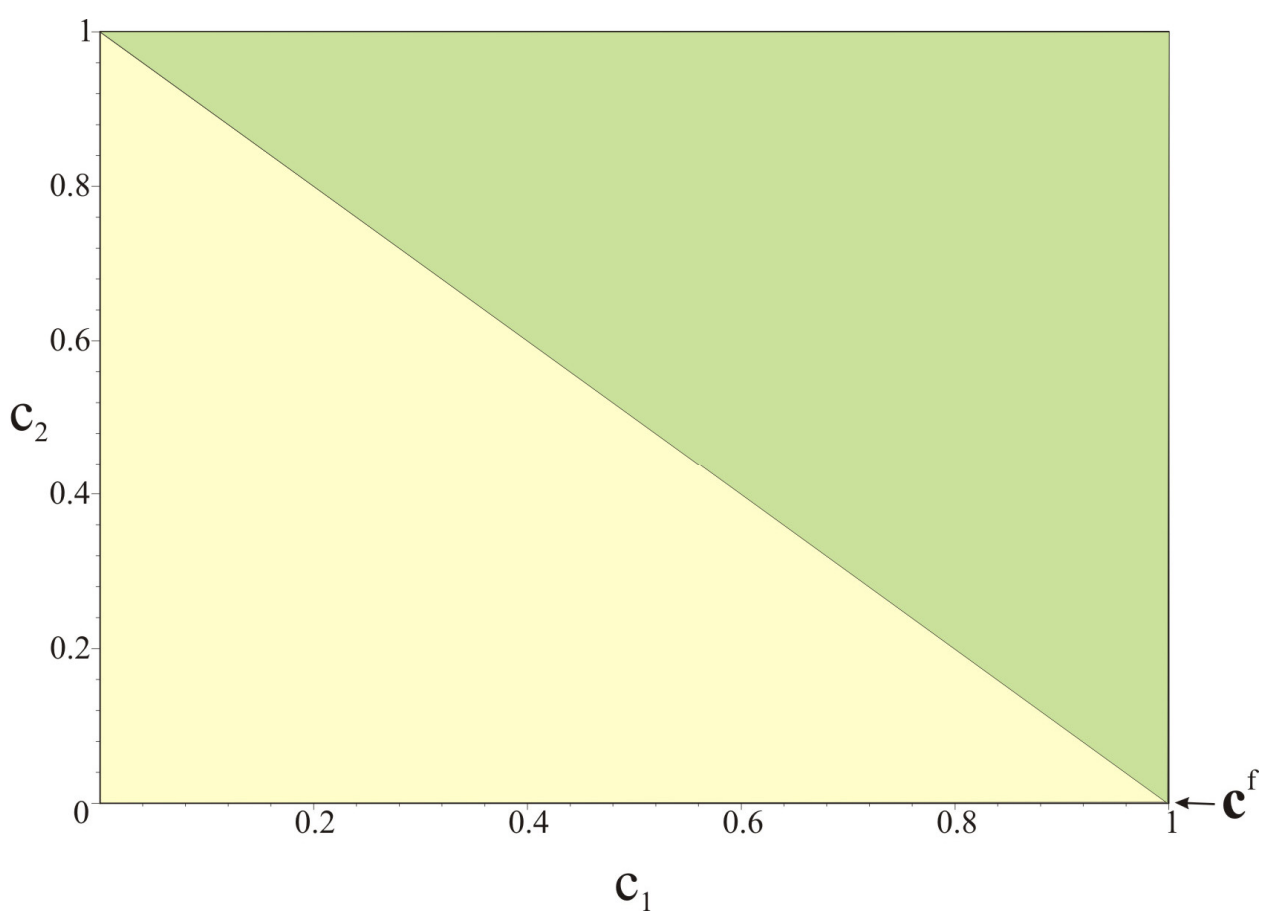

Figure 7.2 Plot showing compositions that are stoichiometrically compatible with the feed for the 2D VdV problem

Figure 7.2 shows those compositions that are constrained by the inequalities in equation (7.4) colored in yellow (lighter shade) while compositions outside 
the constraints are colored in green (darker shade). As discussed in Chapter 5 and 6, these constraints are the starting point for our calculations.

In Figure 7.3 we show the stage-by-stage iteration process in the Maple implementation. Starting with the stoichiometric constraints (based on the given feed composition) we add hyperplanes at each iteration following the techniques described in Chapter 6. One can clearly see how each newly added hyperplane serves to trim a set of compositions from the current polytope.

As iterations progress, redundant hyperplanes (as defined in Chapter 6) are removed as they no longer play a role in constraining the set of feasible compositions. After the $7^{\text {th }}$ iteration in Figure 7.3 the set of feasible compositions is very small and hence the next few iterations are shown in Figure 7.4.

In Figure 7.4, we can see how more hyperplanes are being added while a hitherto unknown composition set seems to take shape. Regions of such a bounding set that inherently possess more curvature, require the introduction of more hyperplanes that in turn approximate curvature better as their number increases.

The final iteration in Figure 7.5 shows a polytope (in light yellow) found after the introduction of 110 hyperplanes. According to the method of bounding hyperplanes, no attainable composition can lie outside this set. A comparison of these bounds with a set of achievable compositions is made in Figure 7.5. The compositions believed to be the full set of achievable compositions (the putative attainable region) for the $2 \mathrm{~d} \mathrm{VdV}$ problem is superimposed on the set of compositions constrained by the 110 hyperplanes. We can see that the outer bounds approximate the putative attainable region well and this supports the belief that the shown configuration of reactors do give rise to 

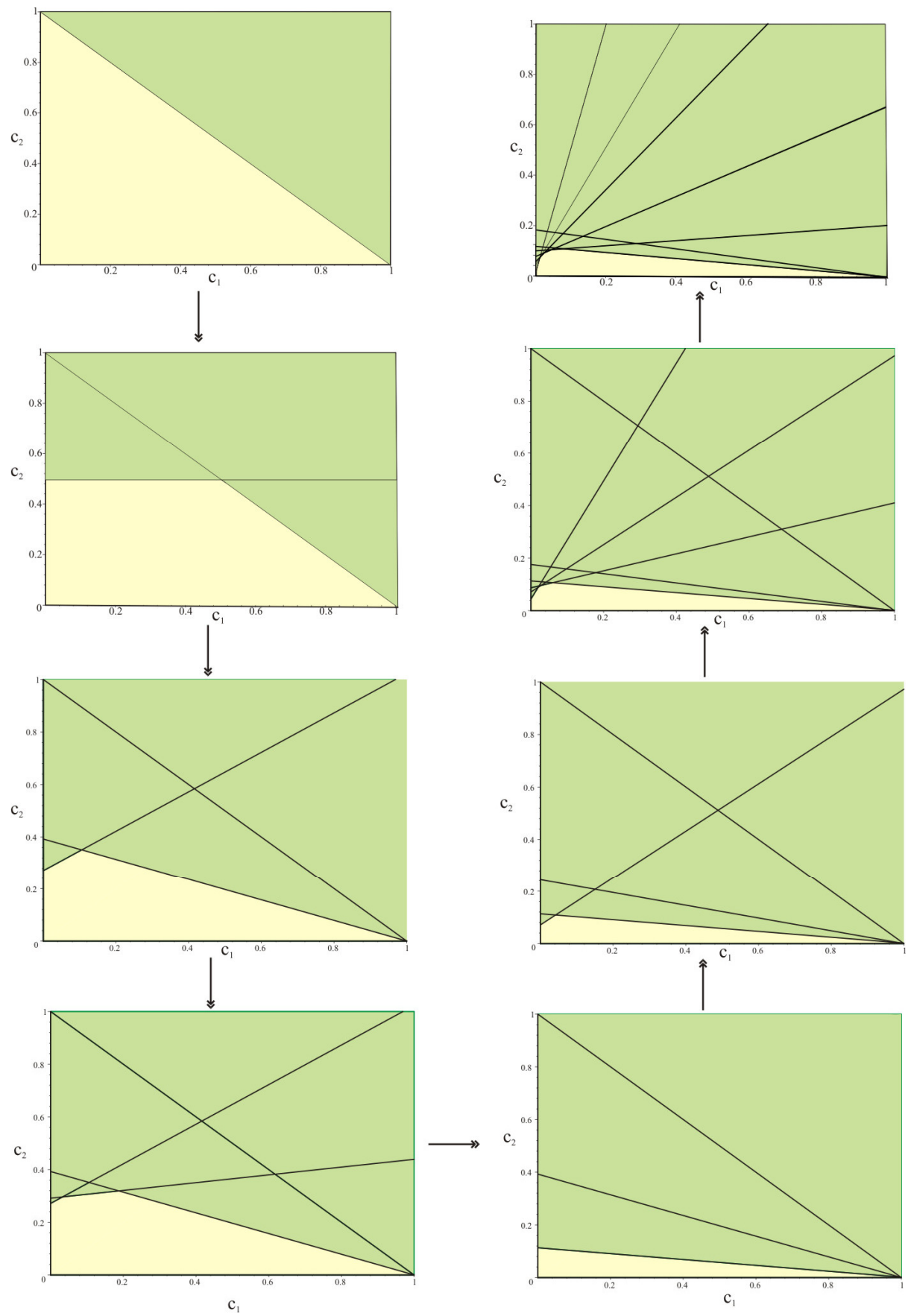

Figure 7.3 Plots showing implementation in Maple of the method of bounding hyperplanes. This is the $2 \mathrm{~d}-\mathrm{VdV}$ example starting with stoichiometric constraints and adding more hyperplanes that trim bounds on achievable composition space 

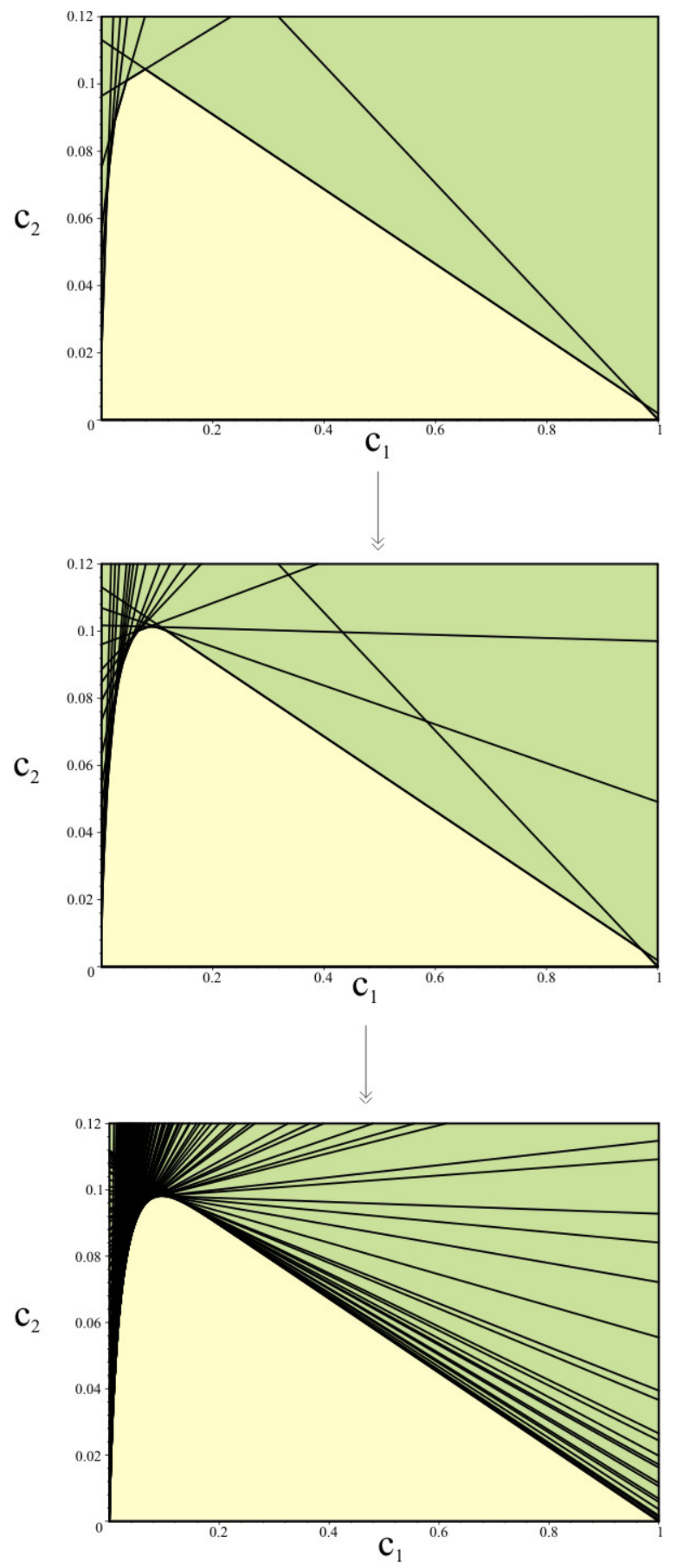

Figure 7.4 Iterations with a large number of hyperplanes showing how more curvature is approximated through more hyperplanes 


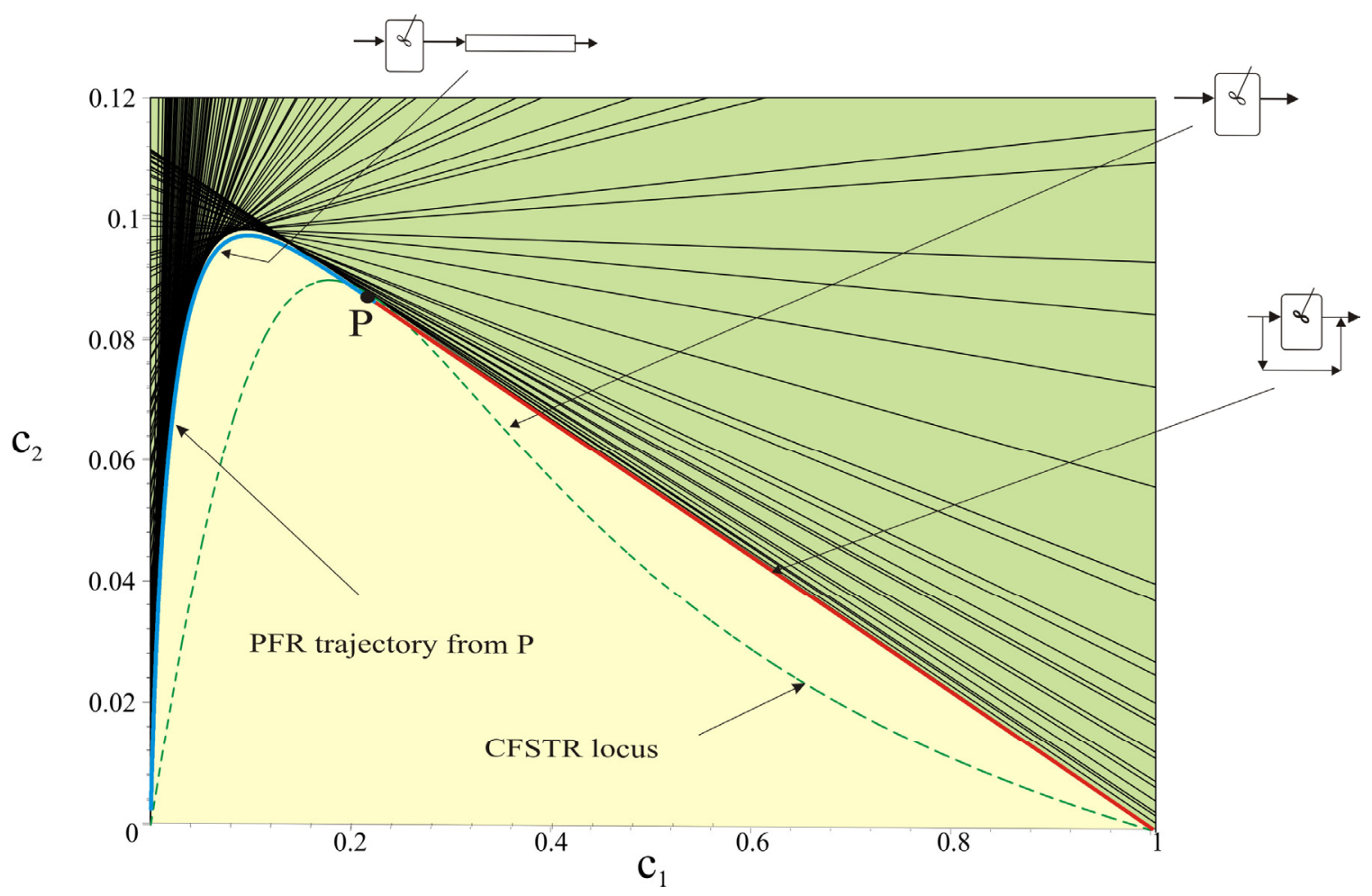

Figure 7.5 Superimposing of two plots (1) Black lines indicating hyperplanes computed by the Maple implementation (2) Curves representing CFSTR loci and PFR trajectories that is believed to be the full set of achievable compositions in the $2 \mathrm{~d} V \mathrm{dV}$ problem

compositions that are the complete set of output compositions for the $2 \mathrm{~d} \mathrm{VdV}$ problem. The results obtained from the Solver DLL implementation are shown in Figure 7.6 and are discussed below.

Figure 7.6 shows the computational results from the Solver DLL implementation superimposed with the compositions believed to be the full set of achievable compositions for the $2 \mathrm{~d} \mathrm{VdV}$ problem. There are 87 hyperplanes that bound the region shown and it seems to approximate the full set of achievable compositions better than the Maple implementation shown in Figure 7.5. The reason for this is that smarter algorithms were used (as described in Chapter 6) in the computations. The 
weighted-bias method in creating new hyperplanes proved useful in finding hyperplanes that approximated curvature better than in the Maple implementation.

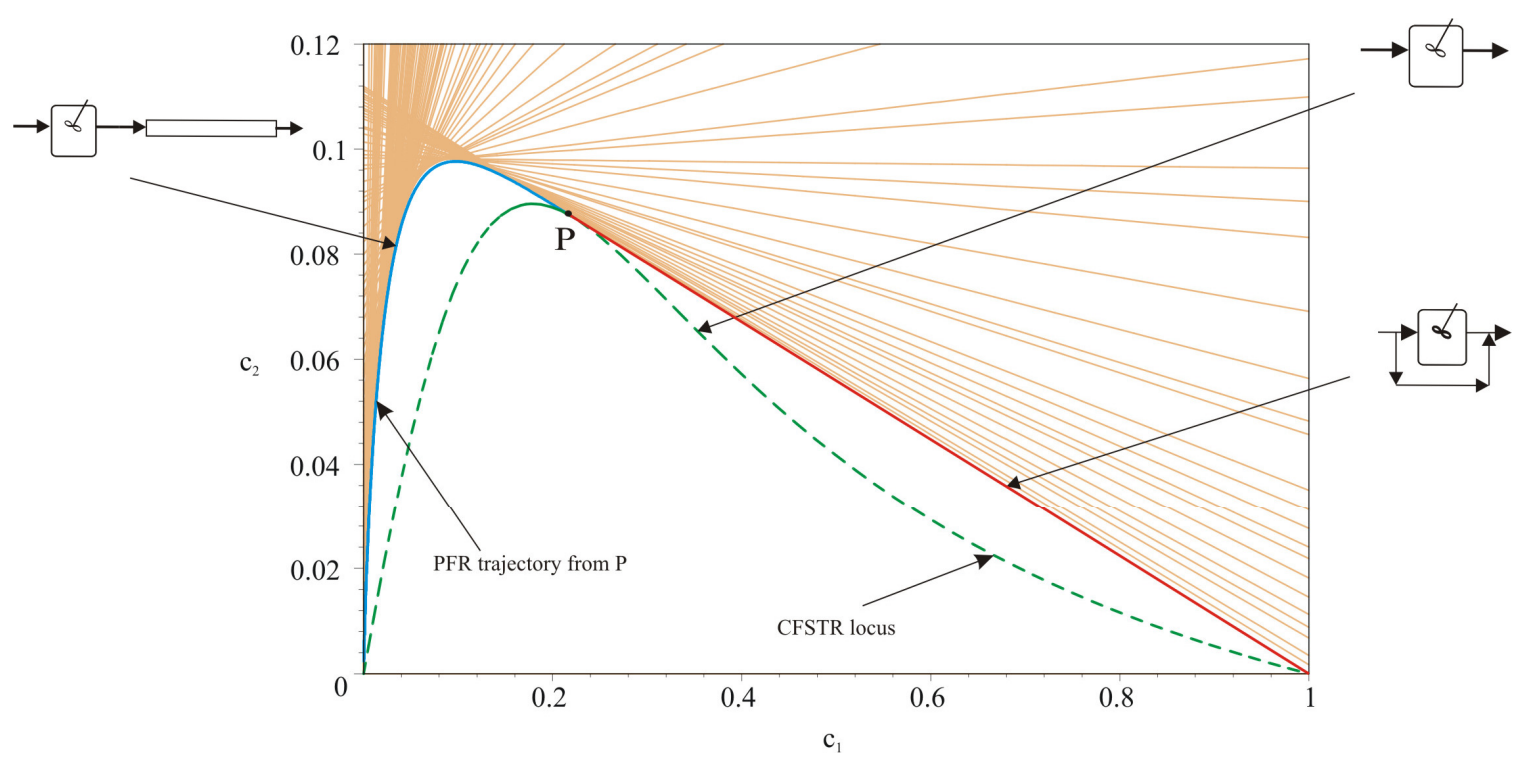

Figure 7.6 Solver Implementation output for the $2 \mathrm{~d}$ VdV problem (cream-brown lines) superimposed on compositions computed for reactor configurations believed to be the full set of achievable compositions.

(B) 3-dimensional VdV: In the 3-dimensional Van de Vusse (3d VdV) problem we are interested in finding the full set of achievable compositions for species $A_{1}, A_{2}$ and $A_{3}$. For the given feed stream composition, the stoichiometric constraints are computed to be the following:

$$
\begin{aligned}
& c_{1} \geq 0 \\
& c_{2} \geq 0 \\
& c_{3} \geq 0 \\
& c_{1}+c_{2}+2 c_{3} \leq 1
\end{aligned}
$$


where the last inequality arises from the equality relationship shown in equation (7.3) and the requirement that $c_{4} \geq 0$.

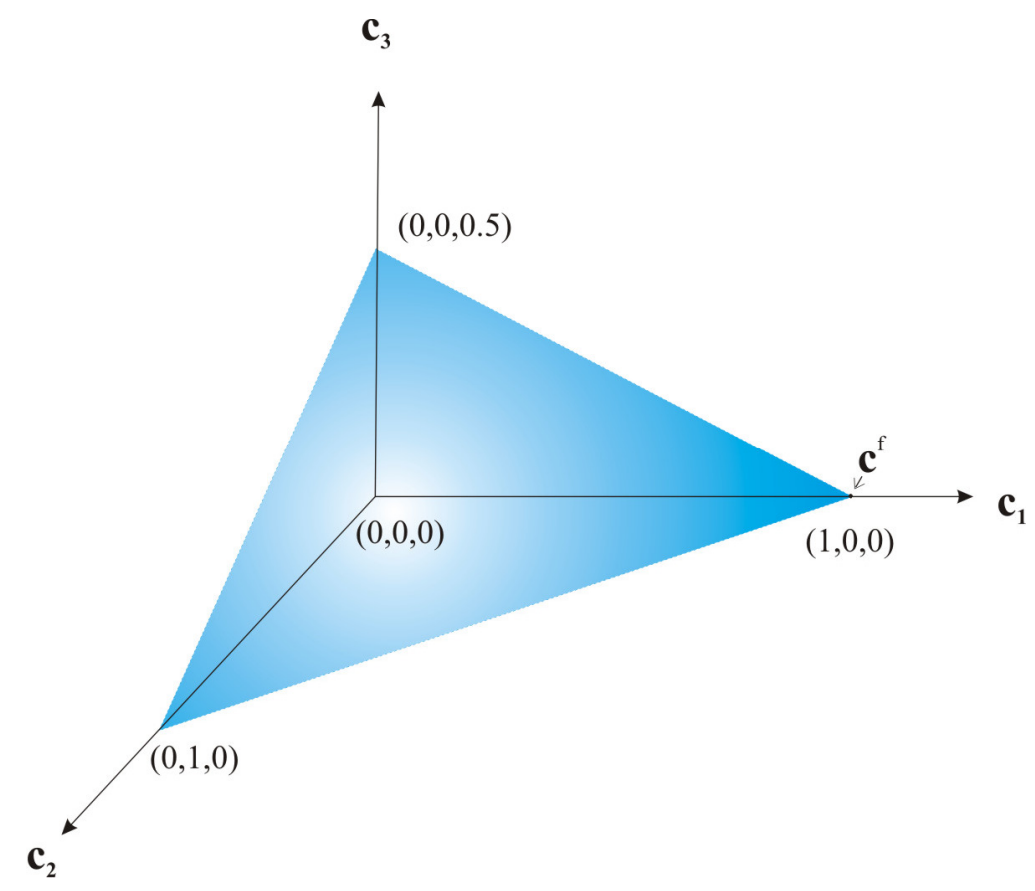

Figure 7.7 Tetrahedron of compositions that are stoichiometrically compatible with the feed for the 3D VdV problem

Figure 7.7 shows the set of compositions constrained by the inequalities in equation 7.4. These compositions lie in a tetrahedron as shown in the figure with vertices $(1,0,0),(0,1,0),(0,0,0.5)$ and $(0,0,0)$. We begin our computation with these constraints. The results from the Maple implementation and the Solver DLL implementation are presented and discussed below.

Figure 7.8 shows a set of bounds computed by Maple superimposed on a set of achievable compositions computed by using design equations described in the articles by Feinberg [4-6, 99]. More precisely, the surface plotted in the figure 
encompasses all compositions (the convex hull of all composition vectors) that are achievable through reaction and mixing where the reaction occurs in reactors of specially selected designs. (Note that there is no claim here that these are the best achievable set of compositions.)

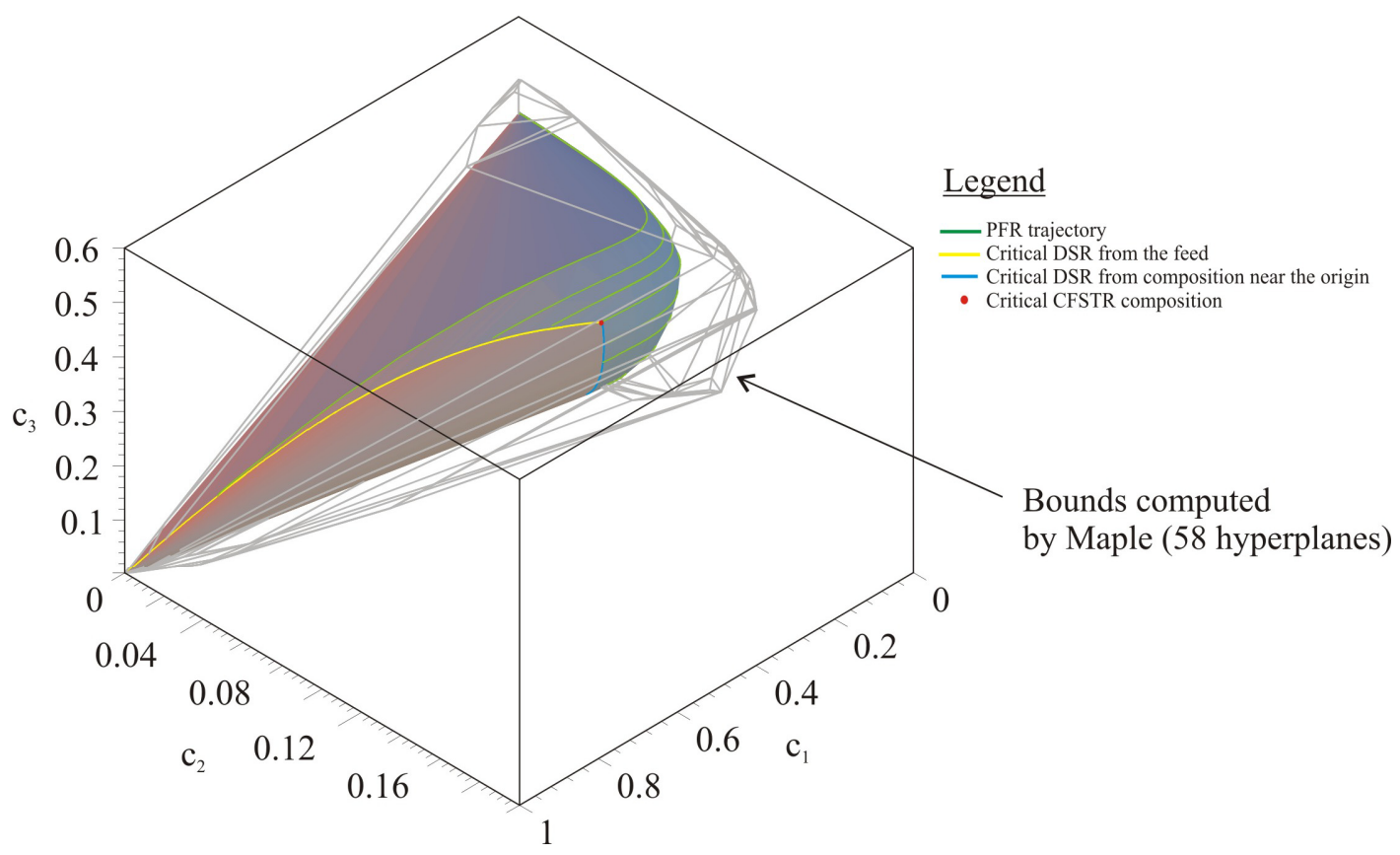

Figure 7.8 Comparison of bounds computed by Maple with compositions from reactor designs suggested by general properties of the attainable region (magnified view)

The bounds shown in Figure 7.8 were computed using the Maple implementation described in Chapter 6. The cage-like structure represents a 58hyperplane bound that was computed for this problem. The computation took several hours to complete and the computational load was also large. Most of the computational load was due to the high grid density that had to be used to obtain accurate results. For 
the Maple implementation, as the number of species being studied increased, the computational load increased considerably. If the grid density were chosen to be 100 , then we would have at most $10^{4}$ valid grid points when we study 2 species and at most 106 valid grid points when we study 3 species and so on. The large data and computations performed on them slowed down the Maple implementation considerably.

On the positive side, Figure 7.9 shows that the bounds computed with the Maple implementation do remove a large chunk of composition space that would otherwise be considered feasible due to stoichiometry. For the given feed stream, the stoichiometrically feasible compositions fill out the tetrahedron outlined by the dotted line. Kinetic bounds are prescribed by the grey cage that constrain compositions that are kinetically feasible

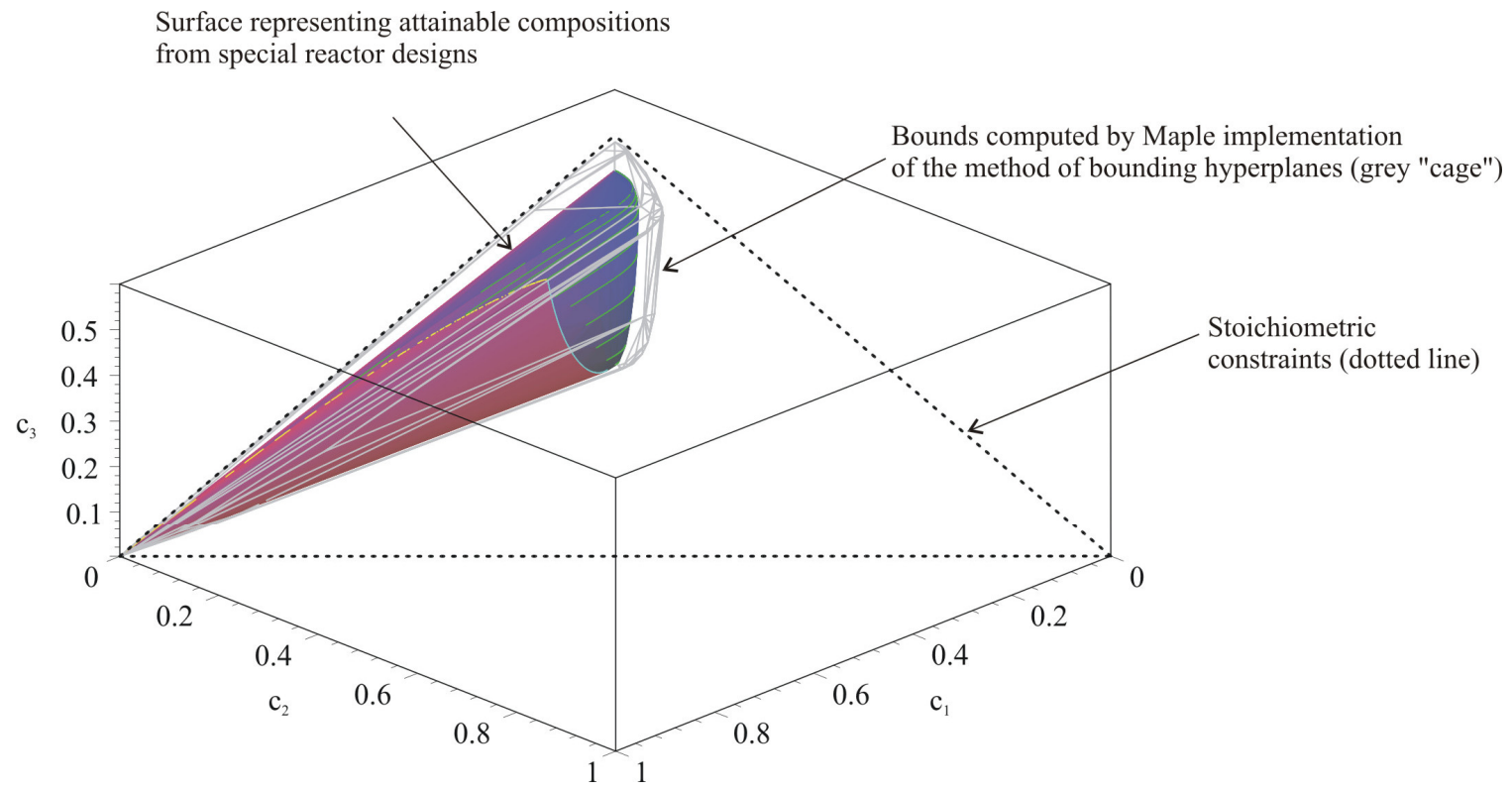

Figure 7.9 Unmagnified view of a set of bounds computed by Maple sandwiched between stoichiometric constraints (dotted line) and a set of achievable compositions computed for the $3 \mathrm{~d} \mathrm{VdV}$ problem 
For a designer, this figure reveals that (for the given reaction system and the given feed) the set of achievable compositions for designs permitting reactions and mixing alone is far smaller than what might be inferred from stoichiometric considerations. Moreover, the bounding cage is sufficiently small as to approach realizable compositions fairly closely, even with 58 hyperplanes.

Analysis along the same lines can be repeated for the $3 \mathrm{~d} V \mathrm{dV}$ example using the Solver DLL implementation in Figures 7.10 and 7.11. Solver DLL implementation computed the 422 hyperplanes shown in Figure 7.10 in less than $25 \%$ of the time taken to compute the 58 hyperplanes computed by the Maple implementation. The improvement in the speed of computation can be attributed to three factors : (1) The Solver DLL implementation is executed by what computer programmers refer to as lowlevel programming which gives instructions to a computer more efficiently than a software interface like Maple, (2) We use nonlinear optimization code that is very efficient in scanning through large regions of composition space as described in the implementation section in Chapter 6, and (3) The "smarter" algorithms used increased the efficiency in implementing the method of bounding hyperplanes.

Since we do not know the location of the true attainable region boundary for this problem, it is difficult to evaluate the absolute merit of a set of computed bounds. Relatively speaking, however, it is clear that the bounds shown in Figure 7.10 are tighter than those shown in Figure 7.8. The reason for this can be explained as follows: Even without knowledge of the actual boundary of the attainable region, it has been shown [4] that such a boundary will be made up of smooth manifolds and straight line sections (see Chapter 3, Section 3.3.) In 2 dimensions the manifolds are curves and in 3 dimensions 
these are smooth surfaces. Theoretically, an infinite number of hyperplanes might be required to approximate such a manifold perfectly. It is for this reason we see that as the number of hyperplanes increases in the 2D and 3D VdV problem, we get a better approximation of a curve in 2D and a smooth surface in 3D.

It is important to distinguish between assertions that can be made from results for the $2 \mathrm{D} \mathrm{VdV}$ problem and the $3 \mathrm{D} \mathrm{VdV}$ problem. The bounds constructed by our method for the 2D VdV problem tightly bound those compositions achieved through the chosen reactor configurations. We can therefore conclude that those compositions might represent the full set of achievable compositions. The bounds constructed for the 2D VdV problem allow us to assert that no composition outside it is achievable through designs with reaction and mixing alone.

For the 3D VdV problem, the bounds computed do not bound the known set of achievable compositions extremely well. At least visually, one can see from Figure 7.10 that there are a few compositions within the bounds but outside the set of known achievable compositions. Therefore, we are not able to assert with confidence that the known set of achievable compositions is in fact the full set of attainable compositions for the given problem. It could be the case that a computation with more hyperplanes would give improved bounds on the achievable composition space. The real limitation in attempting to use more hyperplanes in our computation was due to limits set by the vendor on the number of constraints (and hence a limit on the number of hyperplanes) that could be used with Solver DLL. 


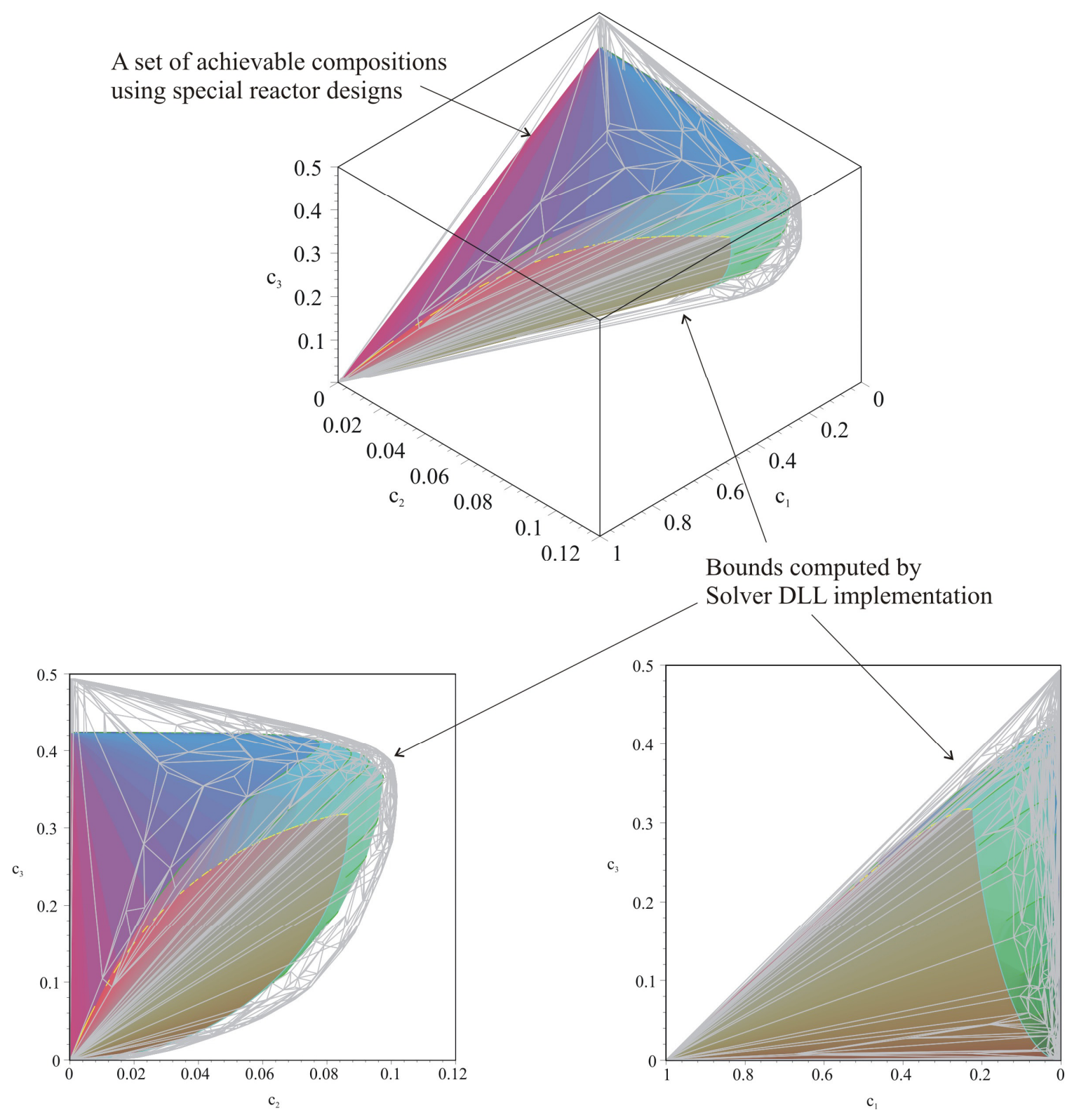

Figure 7.10 Constraints from 422 hyperplanes compared with a set of achievable compositions for the $3 \mathrm{~d} \mathrm{VdV}$ problem as suggested in the literature 


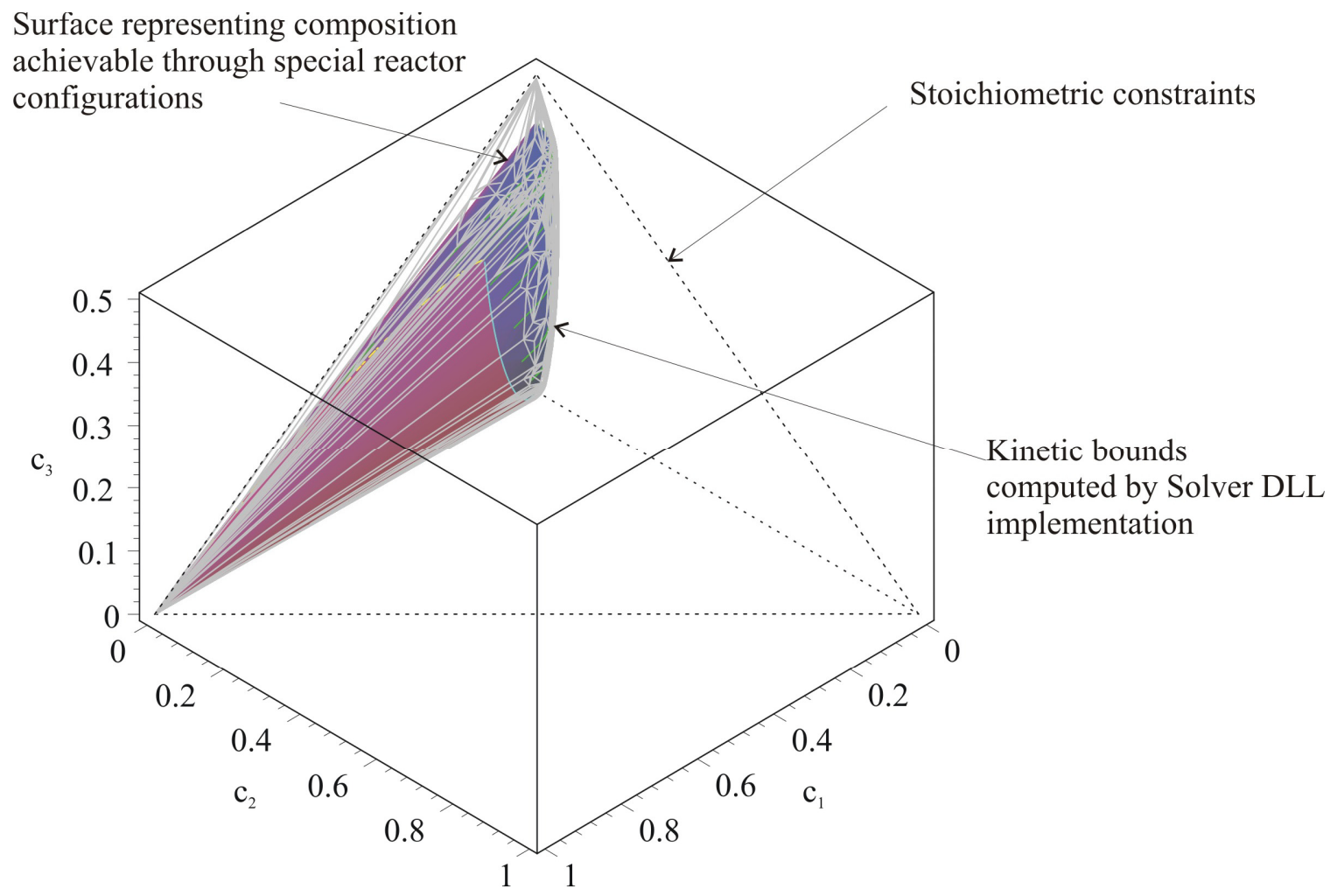

Figure 7.11 Unmagnified view of a set of bounds computed by Solver DLL implementation sandwiched between stoichiometric constraints (dotted line) and a set of achievable compositions computed for the $3 \mathrm{~d} \mathrm{VdV}$ problem

Analogous to our discussion of Figure 7.9, we see in Figure 7.11 that the set of compositions a designer can hope to achieve through reaction and mixing for this example is trimmed drastically when compared to compositions that are considered achievable due to stoichiometric considerations. These results show how our method complements rather than replaces existing methods to ascertain the full set of achievable compositions 


\subsubsection{Reversible Van de Vusse example}

The reversible Van de Vusse reaction network is similar to the one described in Section 6.1.1 except that all reactions are slightly reversible. By permitting mild reversibility in the reaction network, our goal was to compare kinetic bounds with the irreversible Van de Vusse example. The reaction network with rate constants can be written as follows:

$$
\begin{aligned}
& \mathrm{A}_{1} \underset{0.01}{\stackrel{1}{\rightleftarrows}} \mathrm{A}_{2} \underset{0.01}{\stackrel{1}{\rightleftarrows}} \mathrm{A}_{4} \\
& 2 \mathrm{~A}_{1} \underset{0.001}{\stackrel{10}{\rightleftarrows}} \mathrm{A}_{3}
\end{aligned}
$$

The feed stream composition remains the same and can be represented by the vector $\mathbf{c}^{\mathrm{f}}=[1,0,0,0]$. Let $c_{1}, c_{2}, c_{3}$ and $c_{4}$ be the concentrations of species $\mathrm{A}_{1}, \mathrm{~A}_{2}, \mathrm{~A}_{3}$ and $\mathrm{A}_{4}$ respectively. The species-formation rate function can be written as follows

$$
\begin{aligned}
& \mathbf{r}(\mathbf{c}):=\left[-c_{1}+0.01 c_{2}-20 c_{1}^{2}+0.001 c_{3}, c_{1}-0.01 c_{2}-c_{2}+0.01 c_{4}, 10 c_{1}^{2}-0.001 c_{3},\right. \\
& \left.c_{2}-0.01 c_{4}\right]
\end{aligned}
$$

For the given feed stream composition, we can compute the following constraints on concentrations that are stoichiometrically compatible with the feed.

$$
\begin{aligned}
& c_{1} \geq 0 \\
& c_{2} \geq 0 \\
& c_{3} \geq 0 \\
& c_{1}+c_{2}+2 c_{3} \leq 1 \\
& c_{4}=1-c_{1}-c_{2}-2 c_{3}
\end{aligned}
$$




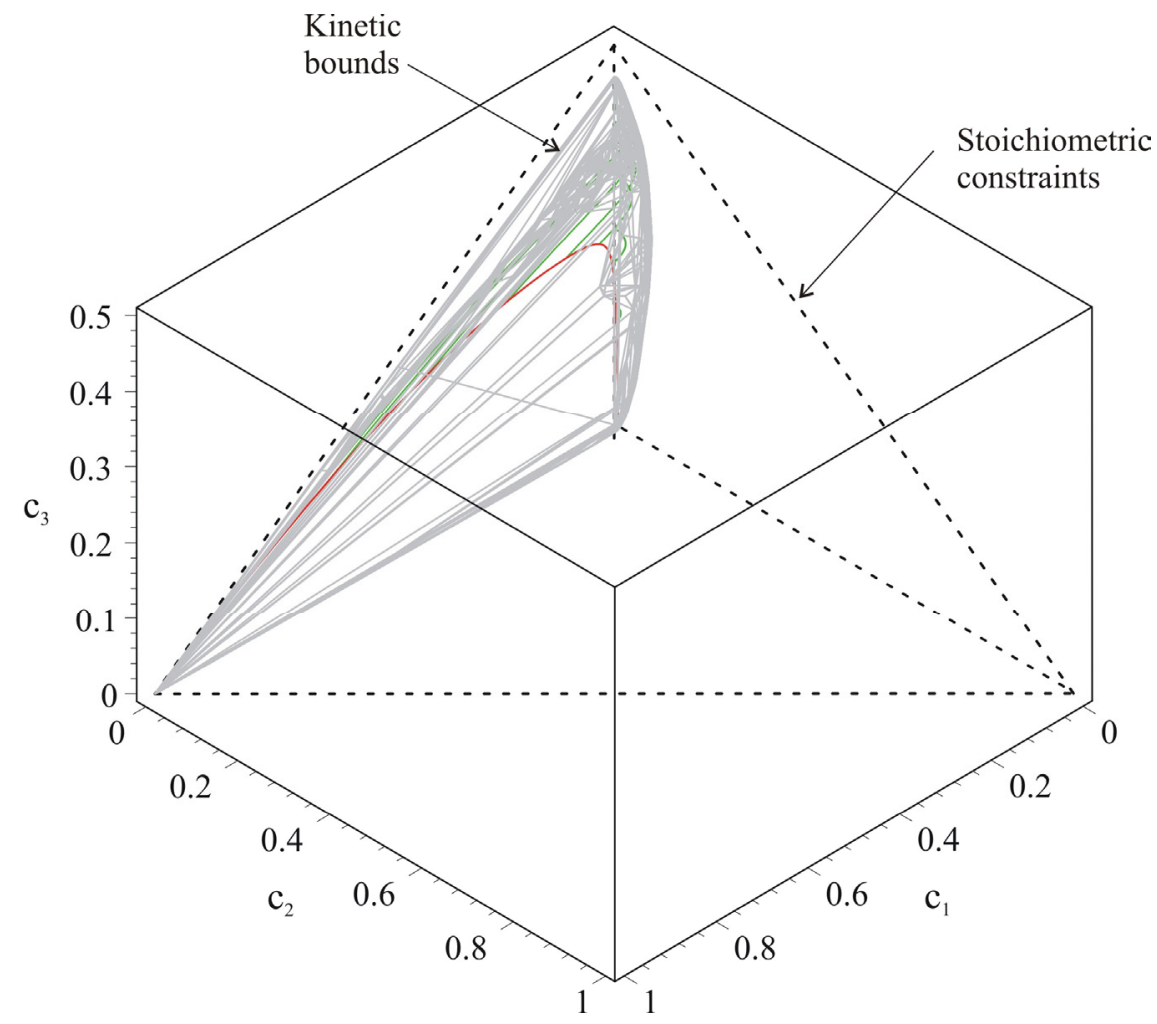

Figure 7.12 Comparing stoichiometric constraints, kinetic bounds and a set of achievable compositions for the reversible Van de Vusse reaction network

Figure 7.12 shows the results obtained in the Solver DLL implementation for the reversible Van de Vusse reaction network. The kinetic bounds trim away a large number of compositions from among those that are considered stoichiometrically compatible with the feed. Figure 7.13 shows in more detail how the kinetic bounds compare with a set of achievable compositions. The grey surface represents the kinetic bounds, the red curve shows steady-state compositions obtained for various residence times in a CFSTR. The green curves represent PFR trajectories for different inlet compositions. 

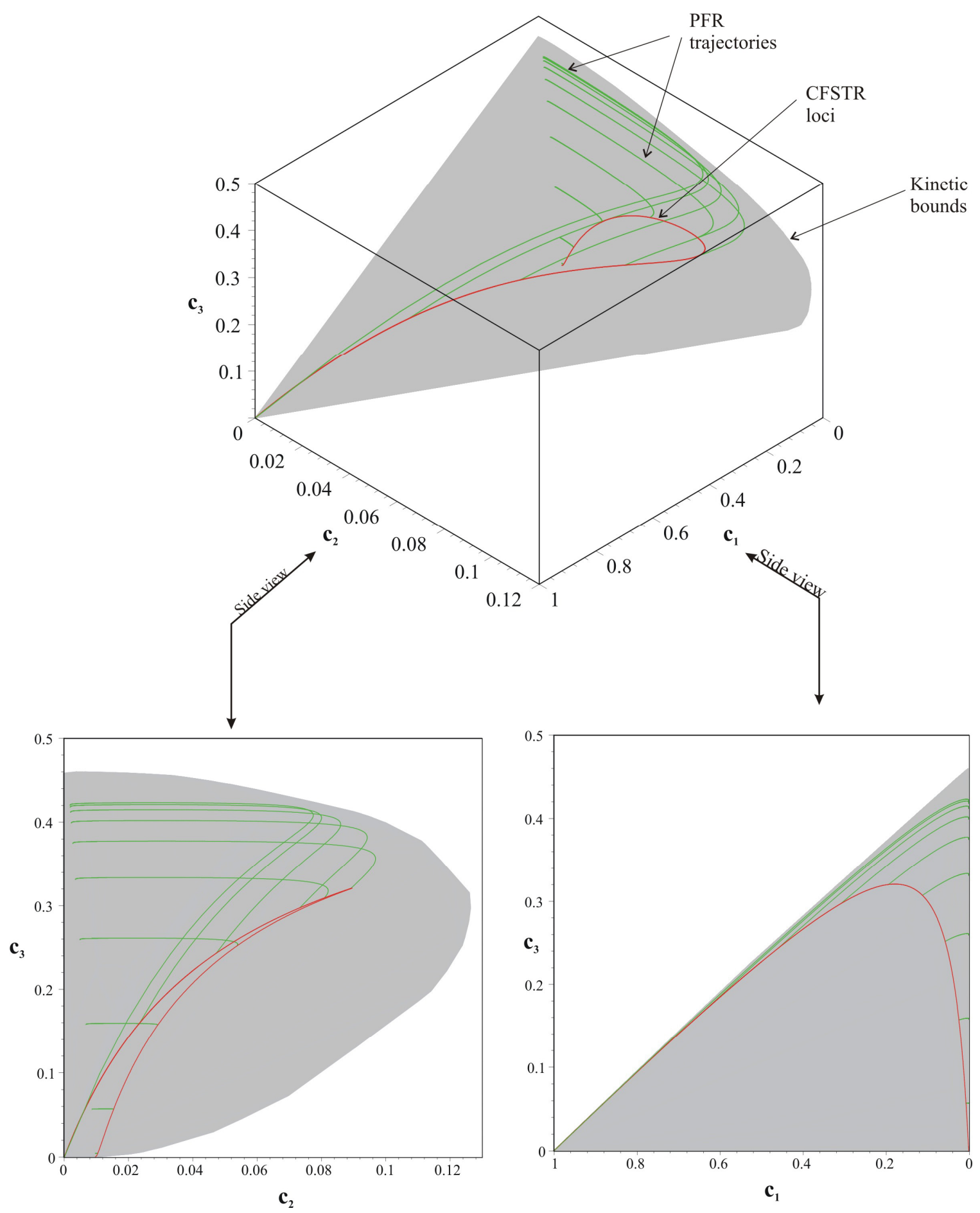

Figure 7.13 Magnified view of the kinetic bounds compared with some achievable compositions computed for various CFSTR residence times (in red) and PFR configurations (in green) 
The bounds obtained are very similar to the irreversible Van de Vusse reaction system since the chosen rate constants make the reactions only mildly reversible. Comparing the unmagnified plots Figures 7.11 and 7.12, we can see that the extent to which compositions are trimmed away from the set considered to be stoichiometrically feasible is similar for the two reaction networks. The difference between the bounds computed is more obvious when comparing the magnified plots Figures 7.10 and 7.13. The bounds computed for the irreversible VdV network seem to be tighter than those computed for the reversible VdV network. Also the bounds computed for the irreversible $\mathrm{VdV}$ network show that the maximum possible value of $c_{3}$ equals 0.5 when $c_{1}=c_{2}=0$ (which is the same as that suggested by stoichiometry). The bounds computed for the reversible $\mathrm{VdV}$ network show that the maximum possible value of $c_{3}$ equals 0.46 when $c_{1}=c_{2}=0$. We believe that the difference observed is due to Solver DLL and probably not due to a difference in the true kinetic bounds.

Our current understanding is that the Solver DLL software struggles to execute optimization near compositions where $\mathbf{n} \cdot \mathbf{r}(\mathbf{c})=\mathbf{0}$ and $\mathbf{r}(\mathbf{c})=\mathbf{0}$ (recall that our objective function comprises of the function generated by $\mathbf{n} \cdot \mathbf{r}(\mathbf{c})$.) This is true for the irreversible $\mathrm{VdV}$ reaction network when $c_{1}=c_{2}=0$ and therefore (by inspecting equation (7.2)) $\mathbf{r}(\mathbf{c})=\mathbf{0}$ for all values of $c_{3}$.

For the reversible $\mathrm{VdV}$ reaction network we see that $\mathbf{r}(\mathbf{c}) \neq \mathbf{0}$ for at least most values of $c_{3}$ when $c_{1}=c_{2}=0$. In this case, Solver DLL does not fail when executing optimizations near compositions where $c_{1}=c_{2}=0$ and is able to successfully trim away kinetically infeasible compositions. This is the reason why the maximum value of $c_{3}$ 
appears to be smaller for the reversible $\mathrm{VdV}$ when compared to the irreversible VdV reaction network. A solution to this problem would require further research into the choice of nonlinear optimization parameters for similar kinds of objective functions.

\subsection{Case study - I}

The following problem was adapted from a separate study on nonisothermal systems for the reactor-separator synthesis problem [100]. In this problem, we have a system of five chemical species $\mathrm{A}_{1}, \mathrm{~A}_{2}, \mathrm{~A}_{3}, \mathrm{~A}_{4}$ and $\mathrm{A}_{5}$. Note that the reaction is similar to the Van de Vusse reaction but with an additional reaction where a reactant combines with one of its products to produce another chemical species. The reactions that occur within the system are as follows

$$
\begin{aligned}
& \mathrm{A}_{1} \stackrel{k_{1}(T)}{\longrightarrow} \mathrm{A}_{2} \stackrel{k_{2}(T)}{\longrightarrow} \mathrm{A}_{4} \\
& 2 \mathrm{~A}_{1} \stackrel{k_{3}(T)}{\longrightarrow} \mathrm{A}_{3} \\
& \mathrm{~A}_{1}+\mathrm{A}_{2} \stackrel{k_{4}(T)}{\longrightarrow} \mathrm{A}_{5}
\end{aligned},
$$

the rate constants are defined as a function of temperature,

$$
\begin{aligned}
& k_{1}(T):=5.23 \times 10^{3} e^{\left(\frac{-11}{R T}\right)} \\
& k_{2}(T):=8.46 \times 10^{8} e^{\left(\frac{-20}{R T}\right)} \\
& k_{3}(T):=2.28 \times 10^{11} e^{\left(\frac{-25}{R T}\right)} \\
& k_{4}(T):=3.56 \times 10^{5} e^{\left(\frac{-12}{R T}\right)}
\end{aligned}
$$

where $R=0.00198 \mathrm{kcal} /(\mathrm{mol} . \mathrm{K})$ is the gas constant and $T$ is the temperature at which the reaction occurs in units of Kelvin. For our isothermal study we selected a temperature of 
$373 \mathrm{~K}$ and evaluated each of the rate constants in eqn (7.7) at that temperature. The species formation rate function is derived using mass-action kinetics as follows:

$$
\begin{aligned}
& \mathbf{r}(\mathbf{c}):=\left[-k_{1}(T) \cdot c_{1}-2 k_{3}(T) \cdot c_{1}^{2}-k_{4}(T) \cdot c_{1} c_{2}, k_{1}(T) \cdot c_{1}-k_{2}(T) \cdot c_{2}-k_{4}(T) \cdot c_{1} c_{2},\right. \\
& \left.k_{3}(T) \cdot c_{1}^{2}, k_{2}(T) \cdot c_{2}, k_{4}(T) \cdot c_{1} c_{2}\right]
\end{aligned}
$$

The feed stream available to our system contains 1 mole per unit volume

of species $A_{1}$ and hence the feed composition can be represented by $\mathbf{c}^{\mathrm{f}}:=[1,0,0,0,0]$.

The following single equality and five inequalities constrain the set of compositions that are stoichiometrically compatible with the prescribed feed.

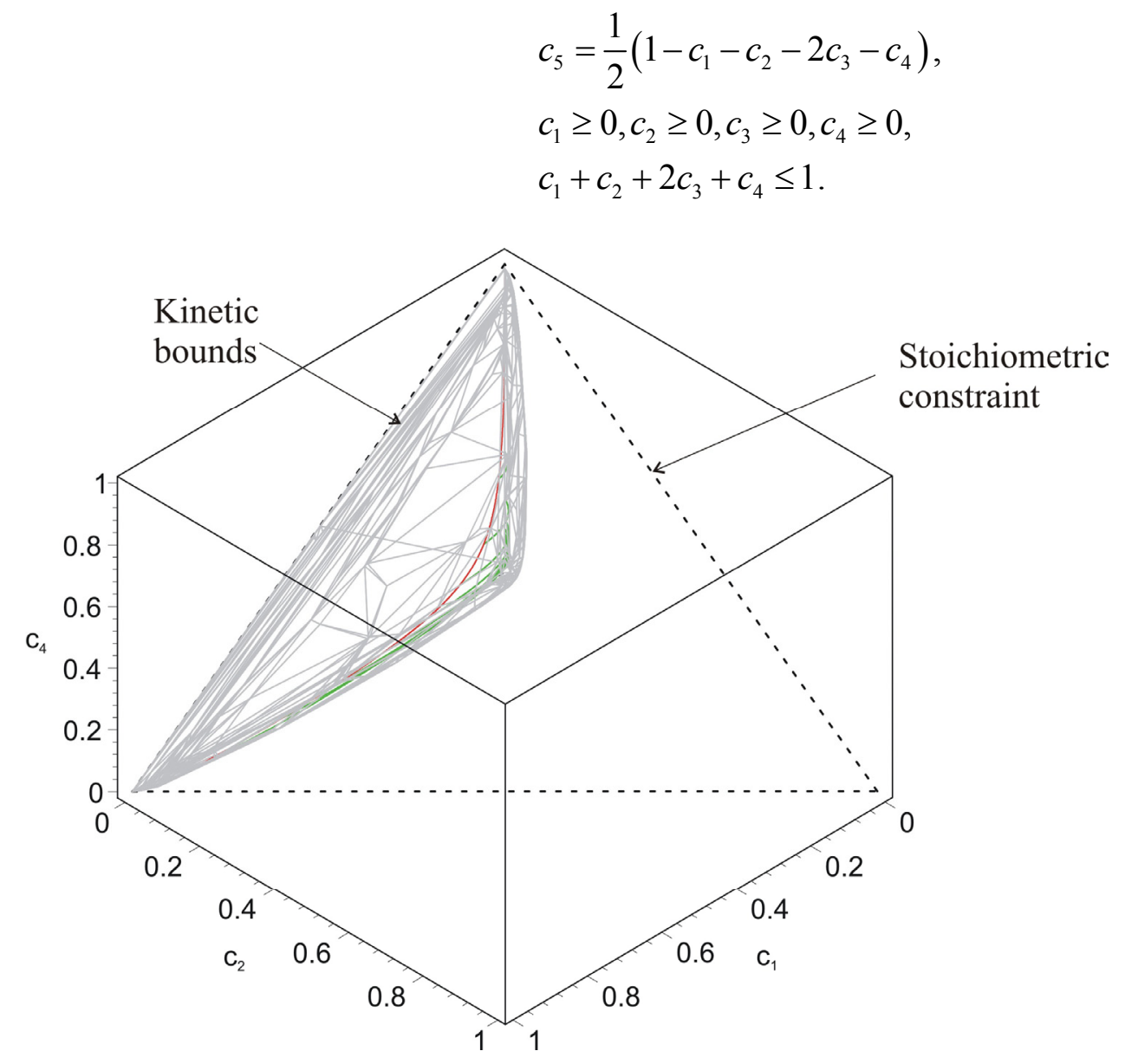

Figure 7.14 Case Study I- Comparing the stoichiometric constraints and kinetic bounds (computed using the Solver DLL implementation) 
From equation (7.8) we can see that it is only required to study the four concentrations $c_{1}, c_{2}, c_{3}$ and $c_{4}$ since $c_{5}$ can be computed uniquely from the given equality. In this case, our Solver DLL implementation will use only 4 variables during optimization.

Figure 7.14 compares the computational results with the stoichiometric constraints for the concentrations of species $\mathrm{A}_{1}, \mathrm{~A}_{2}$ and $\mathrm{A}_{4}$. (Note that we are projecting the convex hull representing the full kinetic bounds into a composition space of smaller dimension.) A large portion of the compositions that are stoichiometrically compatible with the feed are eliminated by the kinetic bounds as infeasible. A magnified view of the kinetic bounds is shown in Figure 7.15 where compositions obtained from CFSTRs for various residence times and PFR composition trajectories are plotted for comparison. The kinetic bounds reveal that the stoichiometric limit of species $\mathrm{A}_{4}$ might be achievable and this is confirmed by the fact that a CFSTR with large residence times does in fact realize this composition.

We have shown two views of the three dimensional plot to get a better idea of how the kinetic bounds constrained by 422 hyperplanes compares with the reactor composition trajectories. Note that the plots in Figure 7.15 have the $c_{2}$ axis magnified to a much larger extent than any other axis. (The $c_{2}$ axis ranges only from 0 to 0.09 while the other axes range from 0 to 1.) For this reason the apparent difference between the grey surface and the colored curves is not as great as the figure suggests.

From the $c_{2}$ versus $c_{4}$ view, we see that the kinetic bounds do not confirm if the reactor configurations chosen give compositions that are the best achievable. In such a case, the designer might have to look at some other reactor configuration that 

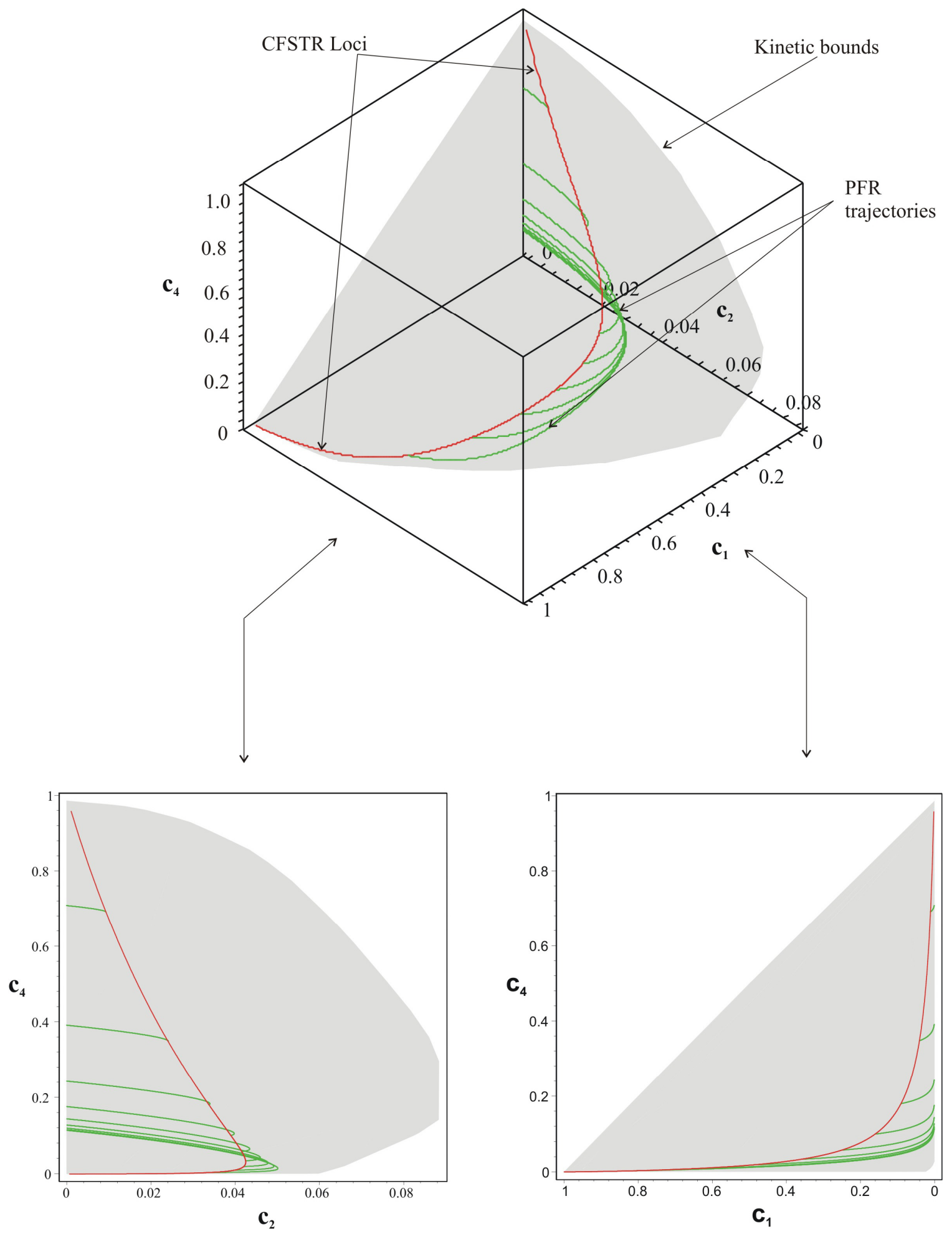

Figure 7.15 Case Study I - Comparing the kinetic bounds with CFSTR loci and various PFR trajectories 
might give a larger achievable composition set. It should also be noted that as the number of species increases, we need more hyperplanes to approximate the kinetically feasible compositions. Hence for this problem with 5-species, the 422 hyperplanes used might not create a tight bound and a better bound would have been obtained if we would have been able to use more constraints in the Solver DLL implementation.

\subsection{Case study - II}

The following problem was adapted from a separate study on nonisothermal systems for the reactor-separator synthesis problem [100]. In this example we look at the following reaction network with 4 chemical species $A_{1}, A_{2}, A_{3}$ and $A_{4}$.

$$
\begin{aligned}
& \mathrm{A}_{1} \stackrel{k_{1}(T)}{\longrightarrow} \mathrm{A}_{2} \\
& 2 \mathrm{~A}_{2} \stackrel{k_{2}(T)}{\longrightarrow} \mathrm{A}_{4}, \\
& 2 \mathrm{~A}_{1} \stackrel{k_{3}(T)}{\longrightarrow} \mathrm{A}_{3}
\end{aligned}
$$

the rate constants are defined as follows

$$
\begin{aligned}
& k_{1}(T):=2.22 \times 10^{3} e^{\left(\frac{-12}{R T}\right)} \\
& k_{2}(T):=5.02 \times 10^{6} e^{\left(\frac{-18}{R T}\right)}, \\
& k_{3}(T):=1.24 \times 10^{8} e^{\left(\frac{-25}{R T}\right)}
\end{aligned}
$$

and the species production rate vector is written as,

$$
\mathbf{r}(\mathbf{c}):=\left[-k_{1}(T) \cdot c_{1}-2 k_{3}(T) \cdot c_{1}^{2}, k_{1}(T) \cdot c_{1}-2 k_{2}(T) \cdot c_{2}^{2}, k_{3}(T) \cdot c_{1}^{2}, k_{2}(T) \cdot c_{2}^{2}\right] .
$$

In equation (7.10), $R$ is the gas constant and $T$ is the temperature at which the reaction occurs. We assume that the reactions take place at $573 \mathrm{~K}$ and evaluate the rate constants 
shown in equation (7.10) at that temperature. The rate constants are then used in equation (7.11) to evaluate the species production rate vector. The feed stream to the system contains species $A_{1}$ at a concentration of 1 mole per unit volume and hence the feed stream composition may be written as $\mathbf{c}^{\mathrm{f}}=[1,0,0,0]$. For the prescribed feed stream, the following inequality constraints and single equality can be derived to describe those compositions that are stoichiometrically compatible with the feed.

$$
\begin{aligned}
& c_{4}=\frac{1}{2}\left(1-c_{1}-c_{2}-2 c_{3}\right) \\
& c_{1} \geq 0 \\
& c_{2} \geq 0 \\
& c_{3} \geq 0 \\
& c_{1}+c_{2}+2 c_{3} \leq 1
\end{aligned}
$$

Using the equality in equation (7.12) we can uniquely compute the concentration for species $\mathrm{A}_{4}$ from the remaining ones and hence it is sufficient to study the concentrations of species $\mathrm{A}_{1}, \mathrm{~A}_{2}$ and $\mathrm{A}_{3}$. Using the Solver DLL implementation we computed kinetic bounds beginning with the stoichiometric constraints shown in equation (7.12) and introduced 1019 hyperplanes that bound the kinetically feasible composition space.

Figure 7.16 show the kinetic bounds computed in comparison with the stoichiometric constraints and a set of achievable compositions. We see that a large set of compositions is trimmed away from those considered stoichiometrically feasible. Figure 7.17 shows the same set of achievable compositions in more detail while Figure 7.18 shows the kinetic bounds in comparison with the same set of achievable compositions. 


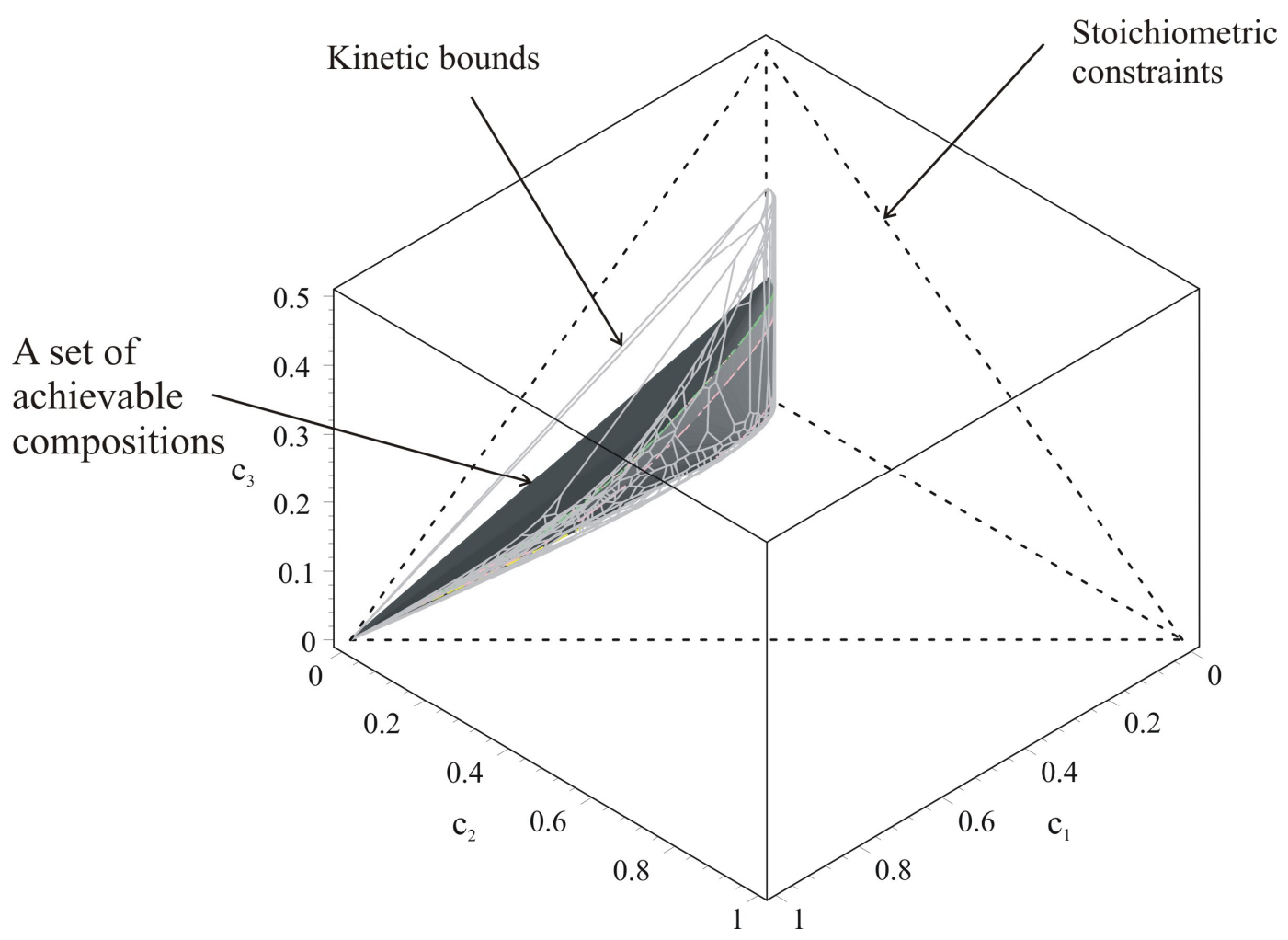

Figure 7.16 Comparison of stoichiometric constraints, kinetic bounds and a set of achievable compositions

The set of achievable compositions used for comparison (shown with more detail in Figure 7.17) was computed in Maple using designs in which the given reaction occurs in combinations of DSRs and PFRs (with mixing). The DSR designs (shown in yellow) were based on formulae derived for critical DSRs [6] that typically shape the boundary of an attainable region. The dark-grey surface is obtained by taking the convex hull of the various possible reactor effluent compositions and hence represents those compositions that can be achieved through mixing also. Note that the surface with more curvature is traversed by DSR and PFR trajectories. 


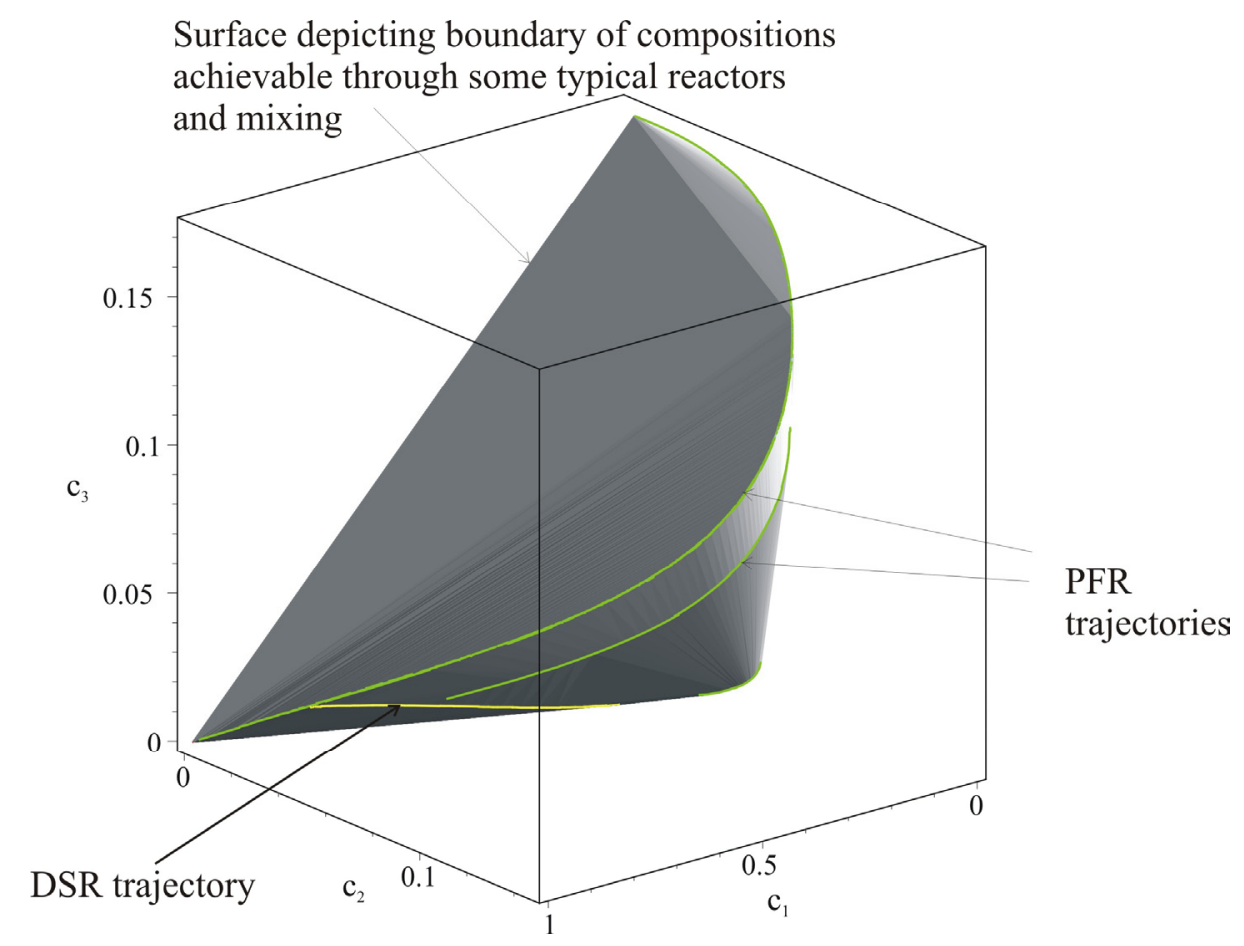

Figure 7.17 A set of achievable compositions with a combination of DSR and PFR trajectories

Figure 7.18 is a magnified view ( $c_{2}$ axis range is only 0 to 0.12 ) showing the comparison between the set of achievable compositions and the kinetic bounds. In this case the introduction of a large number of hyperplanes serves to approximate curvature of the unknown attainable region. The figure confirms that this curvature shares the same features of the critical DSR trajectories plotted. The closeness of the kinetic bounds and the realizable trajectories suggest that with an increased use of hyperplanes to trim the current bounds we might come even closer to the DSR trajectories or perhaps even coincide with it. 


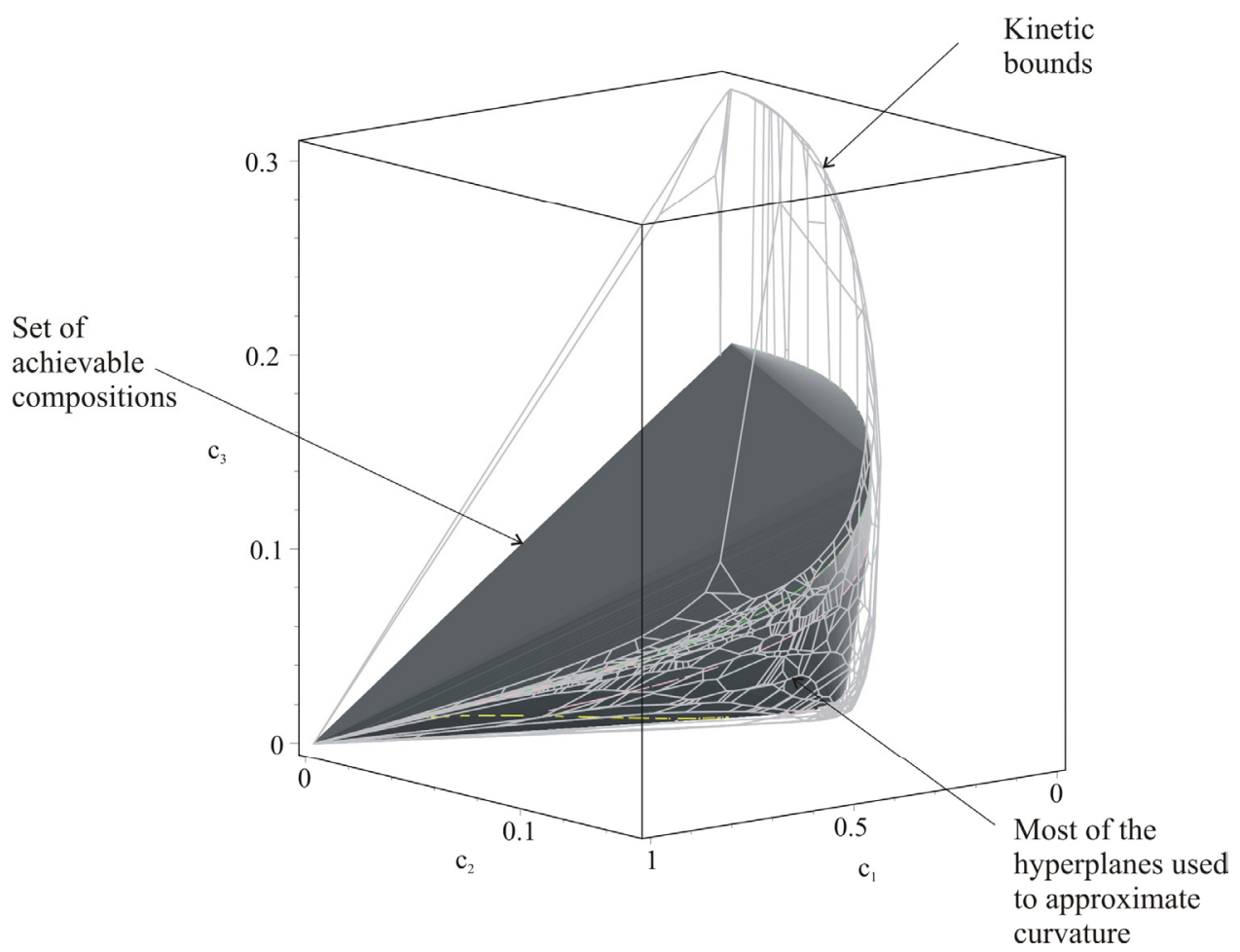

Figure 7.18 Figure showing how more hyperplanes are required to approximate curvature

In Figure 7.18 we see that the kinetic bounds trim infeasible compositions in some areas to a greater extent (such as the region with more curvature) and other regions to a lesser extent (such as the straight-line regions.) In this example we see compositions with larger values of $c_{3}$ are not trimmed away as much as the other species. Aside from optimization problems in Solver DLL, a possible explanation for this could be the fact that $A_{3}$ is a product of reactions involving other species and not a reactant in any other reaction. One might need to remove more infeasible concentrations of its reactants before being able to trim away infeasible compositions with large values of the product. 


\subsection{Case study-III}

The following problem was adapted from a separate study on nonisothermal systems for the reactor-separator synthesis problem [100]. In this example we have four species $A_{1}, A_{2}, A_{3}$ and $A_{4}$ and the reactions occurring in the system are as follows,

$$
\begin{aligned}
& 2 \mathrm{~A}_{1} \stackrel{k_{1}(T)}{\longrightarrow} \mathrm{A}_{2} \\
& \mathrm{~A}_{1} \stackrel{k_{2}(T)}{\longrightarrow} \mathrm{A}_{3} \\
& \mathrm{~A}_{1}+\mathrm{A}_{2} \stackrel{k_{3}(T)}{\longrightarrow} \mathrm{A}_{4}
\end{aligned}
$$

where the rate constants are defined as follows,

$$
\begin{aligned}
& k_{1}(T):=3.6 \times 10^{6} e^{\left(\frac{-12}{R T}\right)} \\
& k_{2}(T):=3.4 \times 10^{4} e^{\left(\frac{-10}{R T}\right)}, \\
& k_{3}(T):=1 \times 10^{5} e^{\left(\frac{-11}{R T}\right)}
\end{aligned}
$$

and the species production rate function can be written in the following manner,

$\mathbf{r}(\mathbf{c}):=\left[-2 k_{1}(T) \cdot c_{1}^{2}-k_{2}(T) \cdot c_{1}-k_{3}(T) \cdot c_{1} c_{2}, k_{1}(T) \cdot c_{1}^{2}-k_{3}(T) \cdot c_{1} c_{2}, k_{2}(T) \cdot c_{1}, k_{3}(T) \cdot c_{1} c_{2}\right]$

In equation (7.14), $R$ is the gas constant and $T$ is the temperature at which the reaction occurs. To study the isothermal characteristics of this reaction we fix the temperature at $373 \mathrm{~K}$ and evaluate the rate constants in equation (7.14) and then substitute them in equation (7.15) to compute the species production rate function. We assume that the feed stream is made up of only species $A_{1}$ flowing into the system at a concentration of 1 mole per unit volume. The feed stream composition may be written as 
$\mathbf{c}^{\mathrm{f}}=[1,0,0,0]$. The species concentrations stoichiometrically compatible with the feed are constrained by the following four inequalities and a single equality.

$$
\begin{aligned}
& c_{4}=\frac{1}{3}\left(1-c_{1}-2 c_{2}-c_{3}\right) \\
& c_{1} \geq 0 \\
& c_{2} \geq 0 \\
& c_{3} \geq 0 \\
& c_{1}+2 c_{2}+c_{3} \leq 1
\end{aligned}
$$

Figure 7.19 compares kinetic bounds for species $A_{1}$ and $A_{3}$ with some achievable compositions using CFSTRs (in red) and PFRs (in green). The kinetically feasible regions, computed by projecting the original bounding polytope to lower dimensions, are unshaded while the infeasible regions are shaded. The kinetic bounds (plotted without any presumption of reactor configurations whatsoever) seem to agree reasonably with a set of achievable compositions computed for this reaction.

An extreme point $\mathbf{c}^{* *}$ of the feasible (white) region residing on the $c_{1}=0$ axis where $c_{3} \simeq 0.138$ provides interesting information (see Figure 7.19.) If $\mathrm{A}_{1}$ is a reactant desired to be consumed completely and $\mathrm{A}_{3}$ is an unwanted side product whose production is to be minimized, then from the kinetic bounds we know that no reactor configuration can consume all of $A_{1}$ while suppressing $A_{3}$ completely in its effluent stream. More precisely, no steady state reactor-only configuration that consumes all of the $A_{1}$ fed into the system can have an effluent stream whose concentration of $A_{3}$ is less than 0.138 mol per unit volume. 


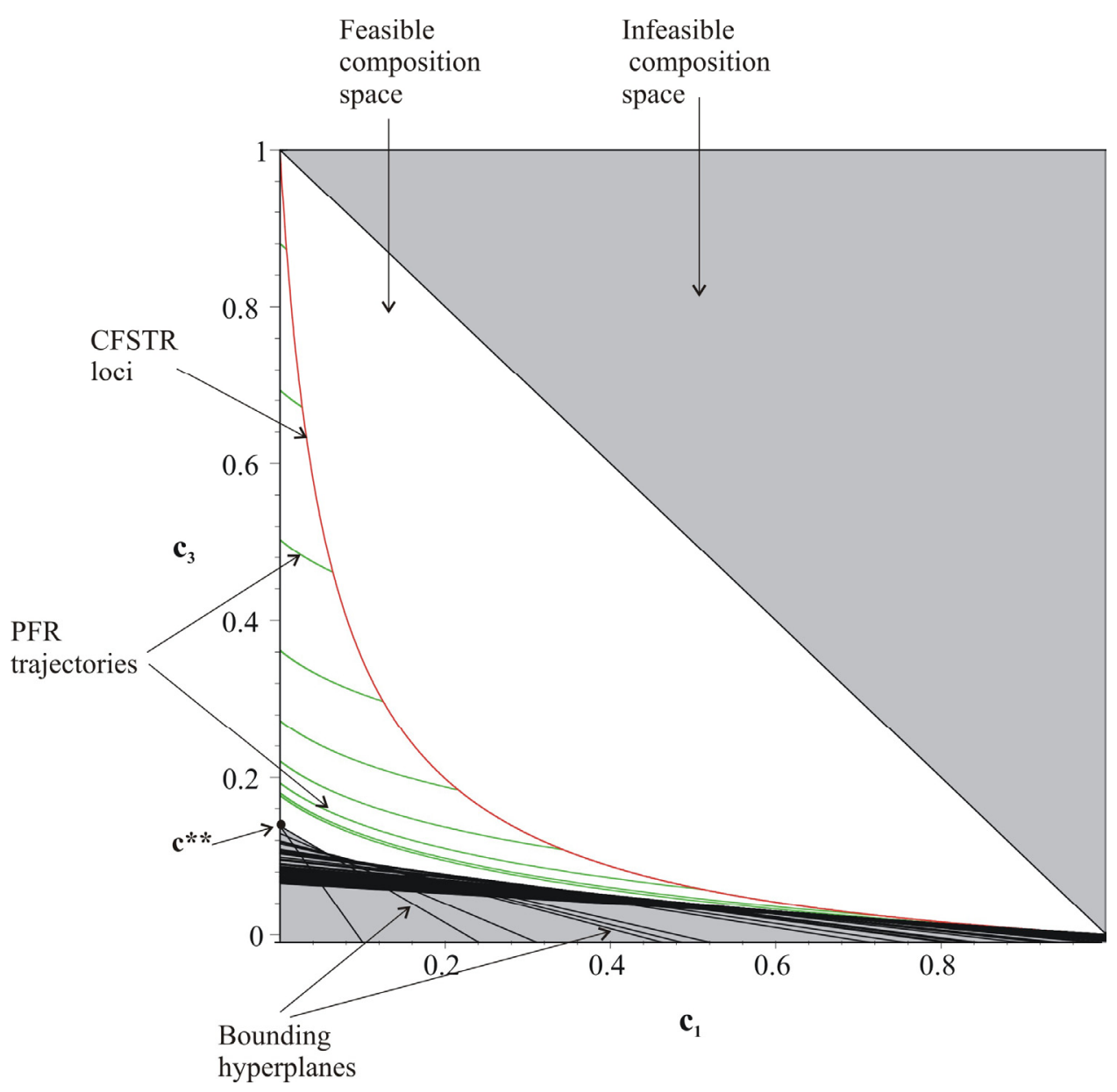

Figure 7.19 A set of achievable compositions in comparison with kinetic bounds computed by Solver DLL implementation

Figure 7.20 compares the stoichiometric constraints, kinetic bounds and a set of achievable compositions for this system. The kinetic bounds do well in revealing what regions of composition space are kinetically feasible and what regions are not. The boundary of a set of achievable compositions computed by using combinations of CFSTRs and PFRs does seem to lie close to the kinetic bounds approximated through 1399 hyperplanes by the Solver DLL implementation. 


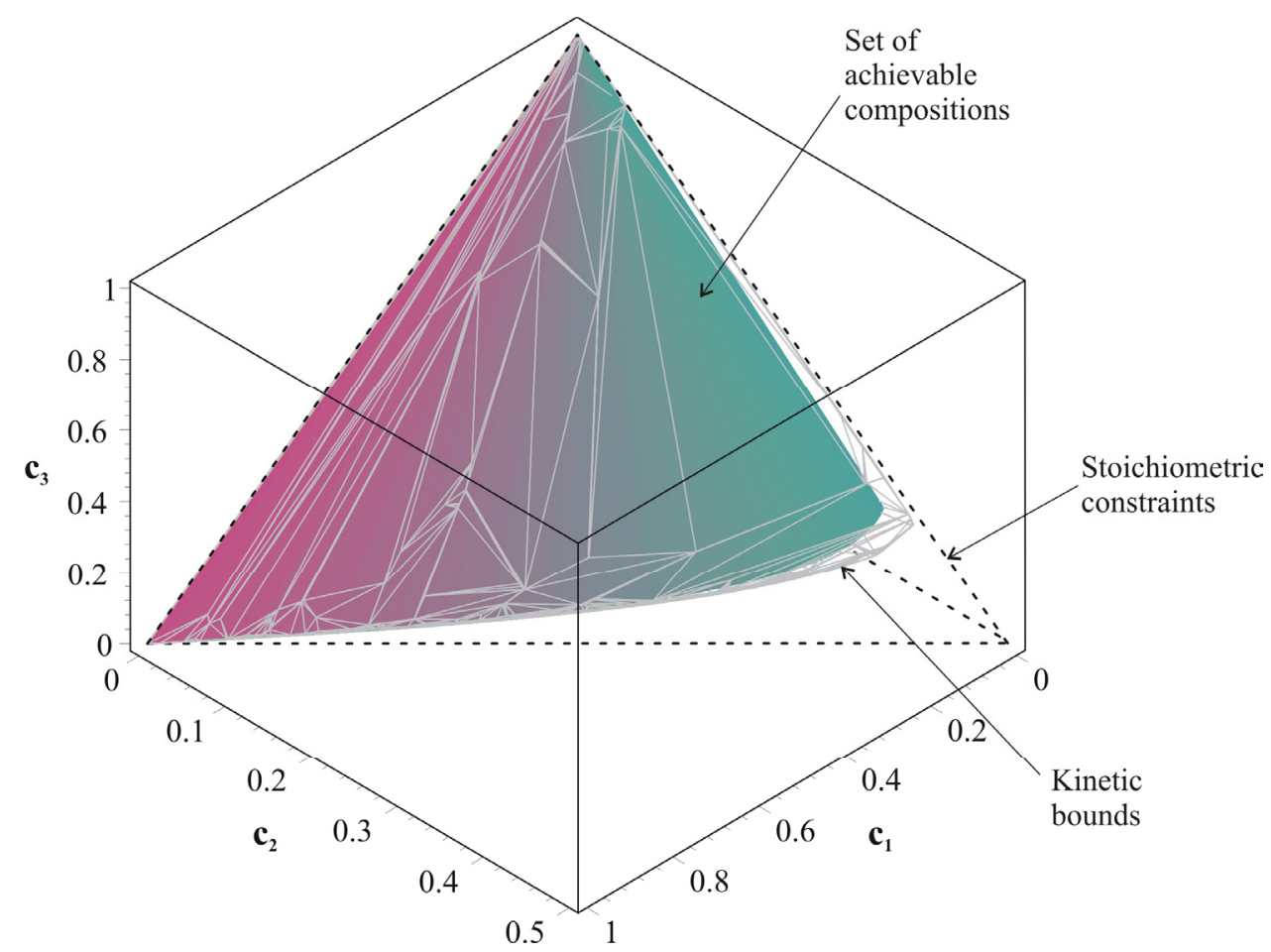

Figure 7.20 Comparison of the stoichiometric constraints, kinetic bounds and a set of achievable compositions

\subsection{Case study- IV : Industrial production of cumene}

Cumene (isopropyl benzene) is produced by the reaction of benzene with propylene. It is commonly used in the industry as an octane enhancer and to produce phenol [101]. The reactions that take place are typically defined for non-isothermal conditions as follows

$$
\begin{aligned}
& \mathrm{C}_{3} \mathrm{H}_{6}+\mathrm{C}_{6} \mathrm{H}_{6} \stackrel{\mathrm{k}_{1}(T)}{\longrightarrow} \mathrm{C}_{9} \mathrm{H}_{12} \\
& \text { propylene + benzene } \rightarrow \text { cumene } \\
& \mathrm{C}_{3} \mathrm{H}_{6}+\mathrm{C}_{9} \mathrm{H}_{12} \stackrel{\mathrm{k}_{2}(T)}{\longrightarrow} \mathrm{C}_{12} \mathrm{H}_{18} \\
& \text { propylene + cumene } \rightarrow p-\text { diisopropylbenzene(DIPB) }
\end{aligned}
$$

The rate constants are functions of temperature and defined as follows 


$$
\begin{aligned}
& \mathrm{k}_{1}(T)=2.8 \times 10^{7} e^{\left(-\frac{12530}{T}\right)} \\
& \mathrm{k}_{2}(T)=2.32 \times 10^{9} e^{\left(-\frac{17650}{T}\right)}
\end{aligned}
$$

The rate constants are in units of liters $/(\mathrm{mol} . \mathrm{sec})$ and $T$ is in Kelvin. The first reaction, whose product is cumene, is the desired reaction while the second reaction, whose product is p-diisopropyl benzene (DIPB), is the undesired reaction. We denote the concentrations of propylene, benzene, cumene and DIPB by $c_{1,} c_{2}, c_{3}$ and $c_{4}$ respectively. In our case study we assume isothermal conditions and pick 563 Kelvin (290 degrees Celsius) as the operating temperature. A different study [100] for this problem suggested that best possible yield of cumene would be achieved close to this temperature. We then compute the rate constants at this temperature. The species production rate vector can be written as

$\mathbf{r}(\mathbf{c}):=\left[-\mathrm{k}_{1}(T) \cdot c_{1} c_{2}-\mathrm{k}_{2}(T) \cdot c_{1} c_{3},-\mathrm{k}_{1}(T) \cdot c_{1} c_{2}, \mathrm{k}_{1}(T) \cdot c_{1} c_{2}-\mathrm{k}_{2}(T) \cdot c_{1} c_{3}, \mathrm{k}_{2}(T) \cdot c_{1} c_{3}\right]$

For this example, we have a feed stream containing 1 mole per unit volume of propylene and benzene each. Hence, the feed composition might be written as $\mathbf{c}^{\mathrm{f}}:=[1,1,0,0]$. The concentrations that are stoichiometrically compatible with the feed are constrained by the following three inequalities and two equations

$$
\begin{aligned}
& c_{3} \geq 0 \\
& c_{4} \geq 0 \\
& c_{3}+2 c_{4} \leq 1 \\
& c_{1}=1-c_{3}-2 c_{4} \\
& c_{2}=1-c_{3}-c_{4}
\end{aligned}
$$

Note that we are interested in plotting the concentrations of the desired product cumene $\left(c_{3}\right)$ versus the undesired product DIPB $\left(c_{4}\right)$. 
The first plot in Figure 7.21 shows the set of compositions that are stoichiometrically feasible with the feed composition (the feed point in this case is $c_{3}=0$ and $c_{4}=0$ shown in the plot). The second plot shows how the kinetic bounds have drastically trimmed down the set of achievable compositions and shows how most of the stoichiometrically feasible compositions are kinetically infeasible according to our computation.

In Figure 7.22, a comparison of the kinetic bounds is with some feasible compositions realized by some CFSTR and PFR configurations. The locus of CFSTR points (shown in red) are effluent compositions from a CFSTR for various residence times including ones that are extremely large. The PFR trajectories represent possible effluent compositions from PFRs with various inlet streams such as the feed stream to the overall system and streams that result from CFSTRs operating at various residence times. For each of the chosen inlet compositions we compute the PFR trajectories (shown as green curves) approaching infinite residence times in the limit.

We can study Figure 7.22 quantitatively to find the maximum concentration of Cumene that can be produced (with the prescribed feed) through any design configuration that permits reaction and mixing alone. From the figure we see that the kinetic bounds prescribe the limit for the concentration of Cumene at $0.962 \mathrm{~mol}$ per unit volume. This is in close agreement with the largest achievable value computed by studying possible effluent compositions of various reactor configurations. In this case a PFR with the feed stream as its inlet gives an effluent stream with a concentration of Cumene $c_{3}=0.95$ (for very large residence times) which is close to the kinetic limit $\left(c_{3}=\right.$ 0.962) prescribed by the method of bounding hyperplanes. 

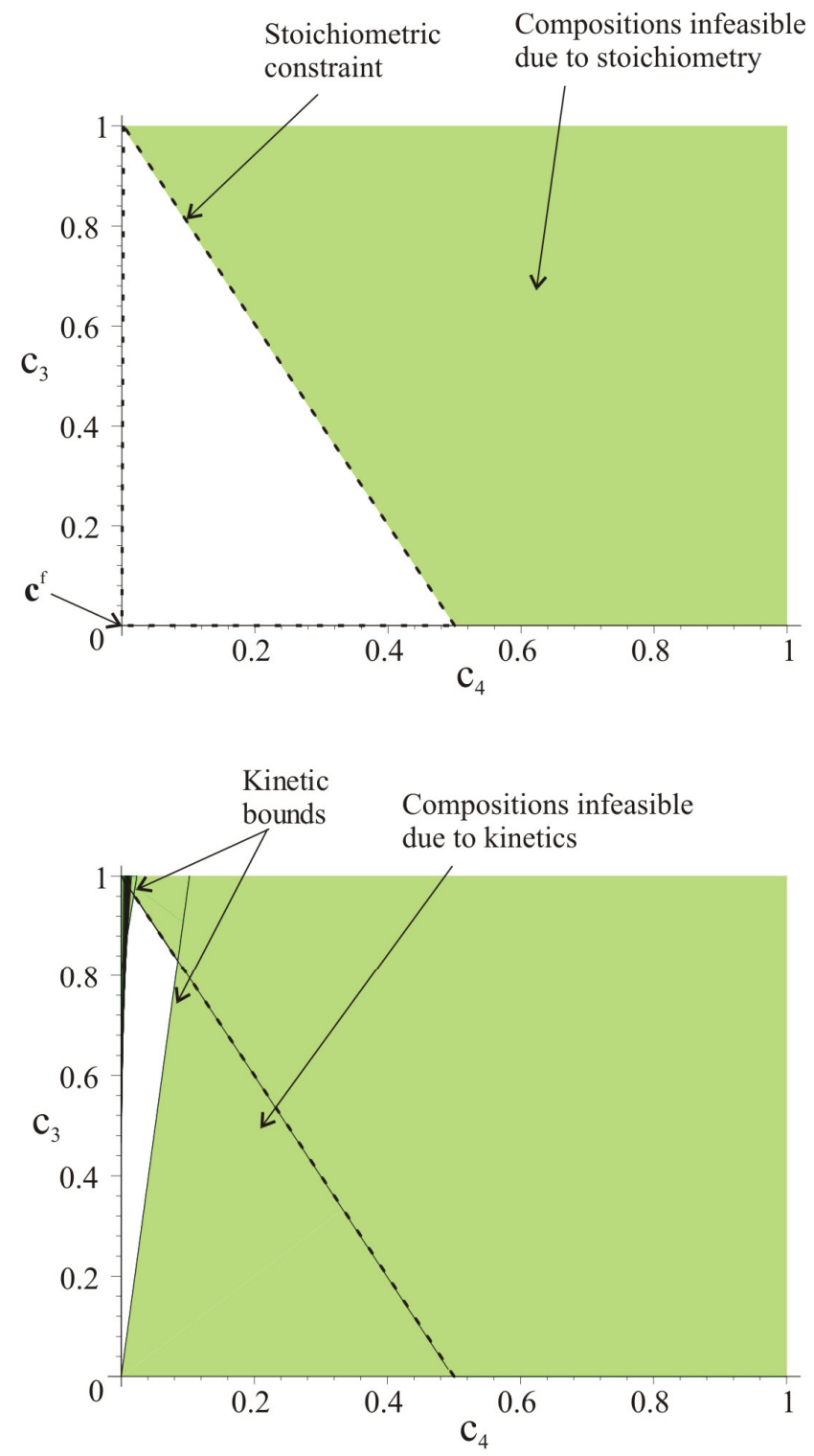

Figure 7.21 First plot showing feed composition and compositions that are stoichiometrically compatible with the feed. Second plot shows kinetic bounds computed by Solver DLL implementation 


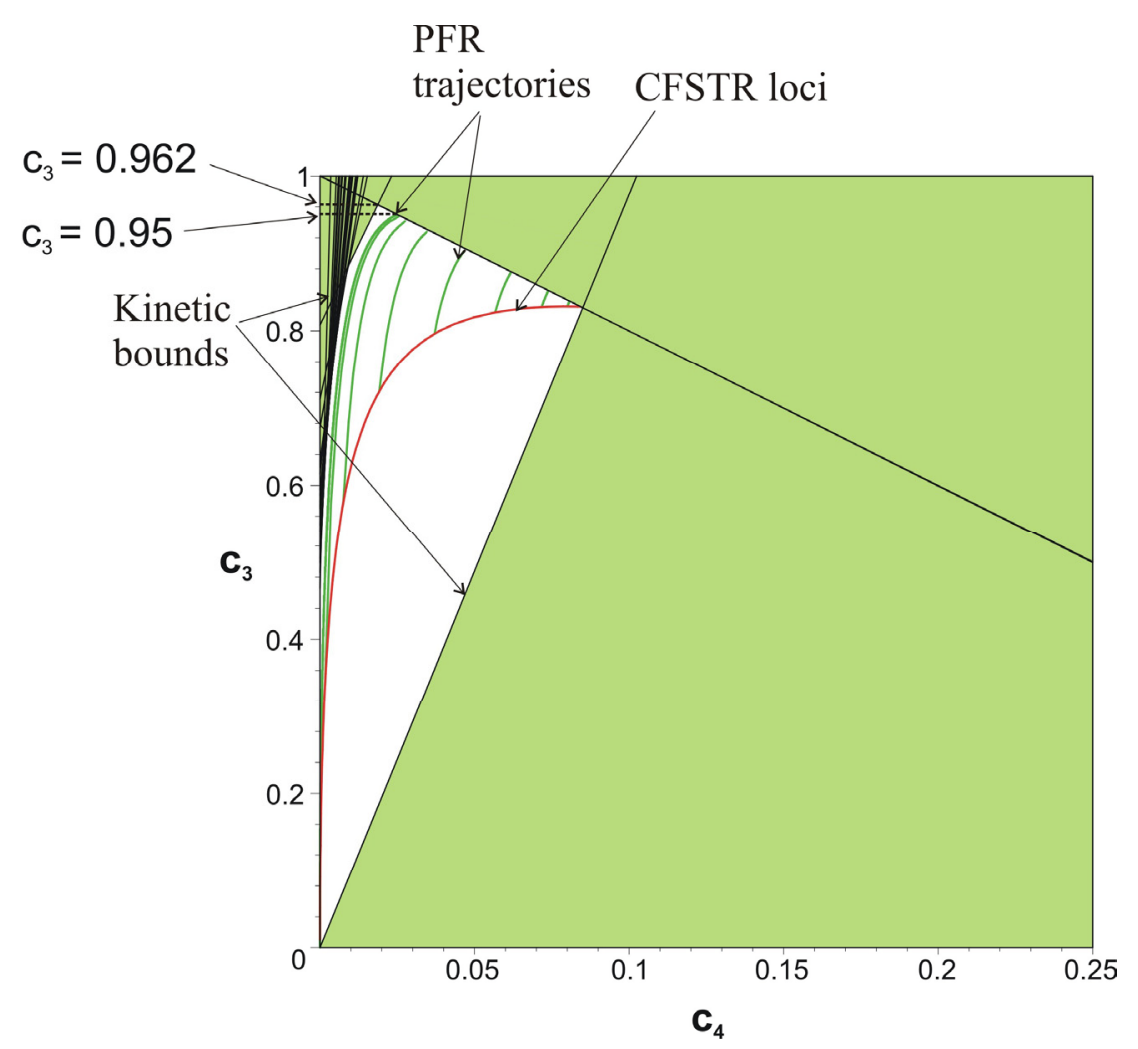

Figure 7.22 Comparing kinetic bounds with a set of feasible compositions computed for various CFSTR and PFR designs

\subsection{Case study V}

The reaction network to be studied in this section is similar to the network studied in Case Study III but we have two more reactions and a total of six chemical species in the reaction. This is an example with the largest number of chemical species for which kinetic bounds were computed. The goal of this case study was to see how the limited ability to add new hyperplanes would effect computing kinetic bounds for large systems. 
The reactions occurring within the system are

$$
\begin{aligned}
& 2 \mathrm{~A}_{1} \stackrel{k_{1}(T)}{\longrightarrow} \mathrm{A}_{2} \\
& \mathrm{~A}_{1} \stackrel{k_{2}(T)}{\longrightarrow} \mathrm{A}_{3} \\
& \mathrm{~A}_{1}+\mathrm{A}_{2} \stackrel{k_{3}(T)}{\longrightarrow} \mathrm{A}_{4} \\
& \mathrm{~A}_{3}+\mathrm{A}_{4} \stackrel{k_{4}(T)}{\longrightarrow} \mathrm{A}_{5} \\
& \mathrm{~A}_{4}+\mathrm{A}_{5} \stackrel{k_{5}(T)}{\longrightarrow} \mathrm{A}_{6}
\end{aligned}
$$

The rate constants are described by the following functions for a non-isothermal system

$$
\begin{aligned}
& k_{1}(T):=8.6 \times 10^{5} e^{\left(\frac{-12}{R T}\right)} \\
& k_{2}(T):=3.4 \times 10^{4} e^{\left(\frac{-10}{R T}\right)} \\
& k_{3}(T):=1 \times 10^{5} e^{\left(\frac{-11}{R T}\right)} . \\
& k_{4}(T):=3 \times 10^{4} e^{\left(\frac{-10}{R T}\right)} \\
& k_{5}(T):=5.1 \times 10^{4} e^{\left(\frac{-11}{R T}\right)}
\end{aligned}
$$

The species production rate vector can be written in the following manner

$\mathbf{r}(\mathbf{c}):=\left[-2 k_{1}(T) \cdot c_{1}^{2}-k_{2}(T) \cdot c_{1}-k_{3}(T) \cdot c_{1} c_{2}, k_{1}(T) \cdot c_{1}^{2}-k_{3}(T) \cdot c_{1} c_{2}, k_{2}(T) \cdot c_{1}-k_{4}(T) \cdot c_{3} c_{4}\right.$, $\left.k_{3}(T) \cdot c_{1} c_{2}-k_{4}(T) \cdot c_{3} c_{4}-k_{5}(T) \cdot c_{4} c_{5}, k_{4}(T) \cdot c_{3} c_{4}-k_{5}(T) \cdot c_{4} c_{5}, k_{5}(T) \cdot c_{4} c_{5}\right]$.

In equation (7.22), $R$ is the gas constant and $T$ is the temperature at which the reaction takes place. We computed the rate constants for a temperature of $373 \mathrm{~K}$ and used the values in equation (7.23) to evaluate the species formation rate vector.

The feed stream to the system contains $1 \mathrm{~mol} /$ unit volume of species $A_{1}$ and hence the feed composition is written as $\mathbf{c}^{\mathrm{f}}=[1,0,0,0,0,0]$. The following six inequalities and single equality constrain compositions that are stoichiometrically compatible with the prescribed feed. 


$$
\begin{aligned}
& c_{6}=\frac{1}{7}\left(1-c_{1}-2 c_{2}-c_{3}-3 c_{4}-4 c_{5}\right) \\
& c_{1} \geq 0 \\
& c_{2} \geq 0 \\
& c_{3} \geq 0 \\
& c_{4} \geq 0 \\
& c_{5} \geq 0 \\
& c_{1}+2 c_{2}+c_{3}+3 c_{4}+4 c_{5} \leq 1
\end{aligned}
$$

Beginning with the constraints in equation (7.24) we use Solver DLL and get a set of bounds made up of 338 hyperplanes. It is obvious that the number of species in this example would demand a large number of hyperplanes to get good kinetic bounds. Limitations in Solver DLL prevented us from using more constraints to trim away composition space.

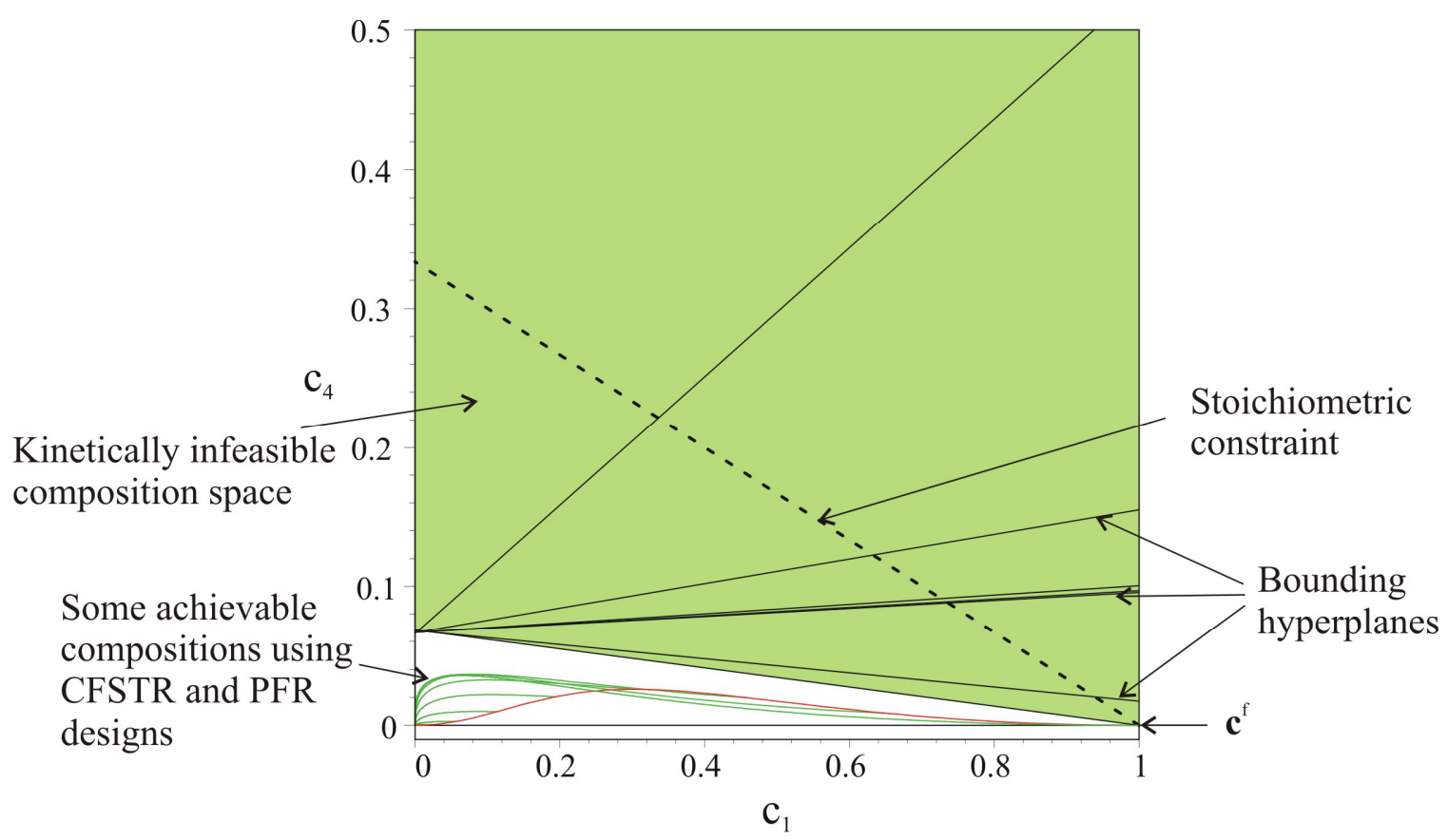

Figure 7.23 Comparing stoichiometric constraints, kinetic bounds and a set of achievable compositions for species $\mathrm{A}_{1}$ versus $\mathrm{A}_{4}$ 


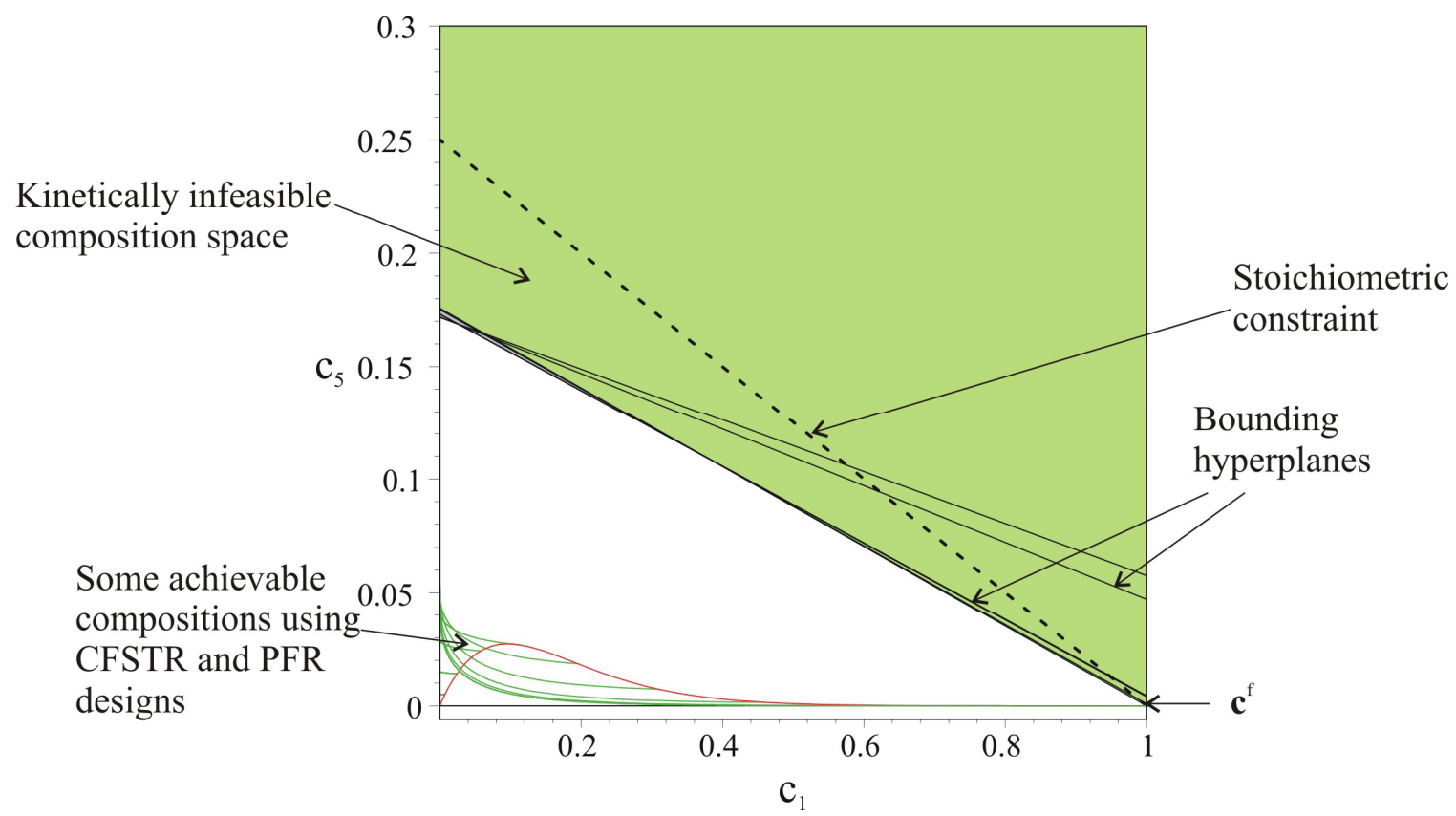

Figure 7.24 Comparing stoichiometric constraints, kinetic bounds and a set of achievable compositions for species $\mathrm{A}_{1}$ versus $\mathrm{A}_{5}$

Figures $7.23,7.24$ and 7.25 are plots showing various projections of the original (six -dimensional) bounding polytope onto different two-dimensional planes. To evaluate the bounds, stoichiometric constraints and a set of achievable compositions are also plotted alongside.

By comparing the number of extreme bounding hyperplanes in Figures $7.23,7.24$ and 7.25 we can see that as the number of hyperplanes increases (in the respective projection) the kinetic bounds seem to be a tighter bound. Figure 7.23 shows bounds on attainable $\left[c_{1}, c_{4}\right]$ compositions and agree reasonably well with the set of realizable compositions plotted. Figure 7.24 shows bounds on attainable $\left[c_{1}, c_{5}\right]$ compositions and agree to a lesser extent with the set of realizable compositions 
plotted. Figure 7.25 seems to suggest that the kinetic bounds computed for species. $A_{6}$ do not reveal much useful information when compared to a set of achievable compositions.

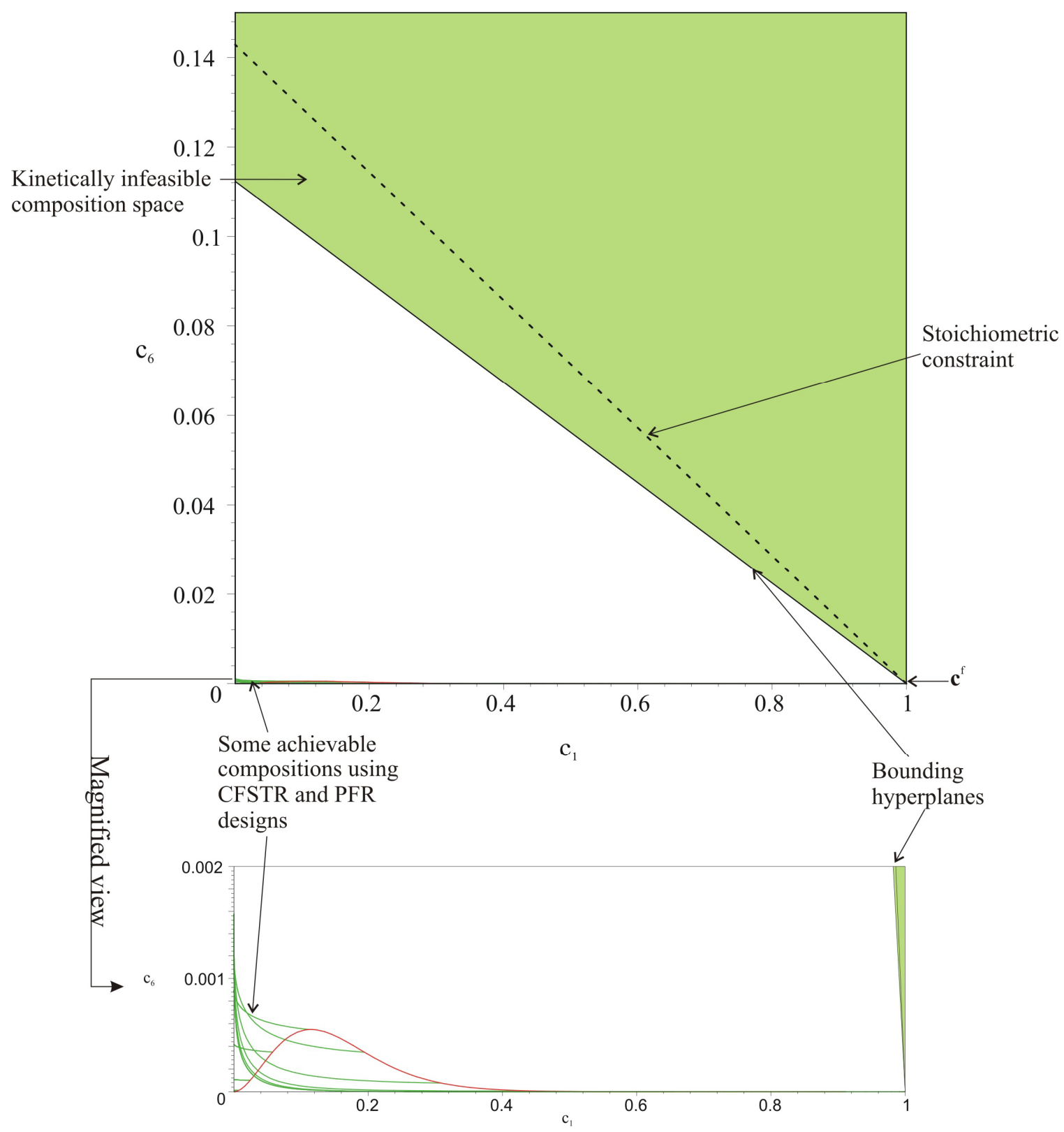

Figure 7.25 Comparing stoichiometric constraints, kinetic bounds and a set of achievable compositions for species $\mathrm{A}_{1}$ versus $\mathrm{A}_{6}$. A magnified view of the achievable composition set is also shown. 
If a very large number of bounding hyperplanes were added during computation then we would have obtained tight bounds for this figure. Also from the reaction network as shown in equation (7.21) one can intuitively see that the full set of attainable concentrations of $\mathrm{A}_{6}$ will arise from the set of attainable concentrations of $\mathrm{A}_{4}$ and $A_{5}$. Therefore, when attempting to find the kinetic bounds for $A_{6}$ we would need to tightly bound the set of attainable concentrations of $\mathrm{A}_{4}$ and $\mathrm{A}_{5}$ (in our iterative method) first before being able to trim away infeasible concentrations of $\mathrm{A}_{6}$. We were unable to do this because of the vendor-imposed limits on the number of bounding hyperplanes that could be used in Solver DLL. In the absence of such a limit we believe that tighter bounds could have been computed for these types of systems where there are large numbers of species. 


\section{CHAPTER 8}

\section{SOME DIRECTIONS FOR FUTURE RESEARCH}

The results presented in Chapter 7 clearly demonstrate the power of the method of bounding hyperplanes. In this chapter, we seek to conclude the thesis by discussing a roadmap of what lies ahead in this approach to process synthesis and design. The various examples described in Chapter 7 suggest that the method of bounding hyperplanes provides practical bounds on compositions that any reactor configuration, however innovative the design, might expect to achieve. It was shown that in some examples the bounds were very sharp and some special reactor configurations approached the bounds well. There are, however, several questions that remain, and they take an inquisitor into different and exciting directions that will be discussed in this chapter.

Broadly speaking, the major issues that need to be addressed can be divided into two: (1) Implementational challenges and (2) Theoretical issues. Of course advances in either of the research areas are will lead to new challenges in the other. 


\subsection{Implementation challenges}

We believe that there is a lot of room for improvement in implementing the method of bounding hyperplanes. The implementation techniques described in Chapter 6 and the computational results shown in Chapter 7 serve as a guide for future research in this area.

We begin by looking at the Solver DLL implementation and describe some improvements that might be made to improve not only the tightness of the bounds but also reduce computational time.

The cdd module described in Chapter 6 serves to mainly remove redundant inequalities and to compute extreme points. Our experience was that increasing number of hyperplanes and increased number of species in the problem slowed down this part of the computation to a large extent. As the number of hyperplanes increased more redundant hyperplanes were found due to the moving of new hyperplanes. Removal of redundant hyperplanes is a computationally intensive process especially when there are a large number of variables and large number of hyperplanes. The need for removal of redundant hyperplanes was discussed in Section 6.2, and therefore we need to either develop an algorithm for the method of bounding hyperplanes without this step or find a different software tool that will perform this computation more efficiently for a large number of variables and constraints.

Finding the extreme points for a given set of constraints was also found to be a very computationally intensive process. The number of constraints did not seem to affect the computational load as much as the number of variables (which correspond to 
the number of chemical species in the problem.) In the area of computational geometry, it is generally accepted that computing the convex hull in higher dimensions (i.e. more variables) is computationally intensive. In addition to the complexity of the task itself, our use of GMP (see Section 6.3) to maintain high accuracy in the computations has also slowed down the computing of extreme points. The accuracy was essential to prevent small errors from entering into various iterative algorithms where the errors magnified giving unacceptable results. There is, therefore, a need to find reliable code (or algorithms) to compute extreme points for a given set of constraints in a faster and more efficient manner, especially for problems with more species (i.e. convex hulls in higher dimensions.)

In the method of bounding hyperplanes, at each extreme point we introduce a new hyperplane in an attempt to trim away infeasible compositions. The normal (or orientation) of this new hyperplane is important in ensuring good trimming of infeasible compositions. Figure 7.5 and 7.6 in Chapter 7 are excellent examples that reveal the importance of the normal computed for the new hyperplane. The bounds in Figure 7.5 were computed in Maple using an equal weight factor to find the normal of a new hyperplane. The bounds in Figure 7.6 were computed in Solver DLL implementation using a biased weight factor to find the normal of a new hyperplane (as detailed in Section 6.3.) The 84-hyperplane bound in Figure 7.6 appears to be a tighter bound than the 110-hyperplane bound shown in Figure 7.5, which means that the hyperplanes chosen in the bias-weighted method did better at trimming away infeasible compositions (for this example). We need to look at more advanced techniques that will help us find a 
hyperplane normal that is most likely to trim away the largest set of compositions that are kinetically infeasible.

With respect to the Solver DLL module, the major improvement we seek is the ability to use more constraints. More constraints mean that the kinetic bounds that are constructed might be tighter. As the number of chemical species increases the number of constraints required to give good kinetic bounds increases tremendously. In the examples shown in Chapter 7 , we can see that about $10^{2}$ hyperplanes bound a set of achievable compositions for the 2D Van de Vusse problem well and for the 3D Van de Vusse problem, $10^{3}$ hyperplanes constrain a set of achievable compositions to a reasonable extent. For most of our examples described in Chapter 7, we were limited by the nonlinear programming tool Solver DLL to using no more than 250 hyperplanes. (In some cases the choice of parameters permitted the use of as many as 1000 hyperplanes, but that choice of parameters sometimes led to failures in the optimization algorithm.)

For a general problem we are not certain about the number of bounding hyperplanes that might be needed for a coarse approximate bound as compared to the number of hyperplanes that might be needed for a tight bound. It is difficult to perform an absolute assessment of the bounds simply because we do not know the true boundary of the attainable region. If we are given the opportunity to use an infinitely large number of bounding hyperplanes it is not known if we will have successfully bounded the true attainable region. This leads to theoretical questions that will be discussed in the next section.

Figure 6.14 shows how constraints are changed in the Solver DLL module to eliminate those regions that are kinetically infeasible. The iterative algorithm used (see 
Section 6.3) requires performing an optimization at every iteration. Minimizing the number of iterations will enhance the speed of computation tremendously.

We have described in Chapter 7 how the intensity of computations increases when the number of extreme points increases. It is for this reason that a scheme was devised (described in Section 6.3) to select extreme points where new hyperplanes would be introduced. This method of selecting extreme points did give limited improvement to the speed of computation. Further investigation is needed into methods that would suggest the best choice of extreme points where introducing new hyperplanes trim away the most compositions that are kinetically infeasible.

The examples discussed in Chapter 7 show that as the number of species increases, our software implementation struggles in giving meaningful outer bounds. A slightly reformulated problem would be to maximize the production of a single species rather than the output compositions of all the species. Instead of attempting to find outer bounds on feasible compositions for all species it may be easier to implement an algorithm that focuses on maximizing a single desired species or minimizing a single undesired species.

\subsection{Theoretical issues}

Besides the challenges associated with trying to implement the method of bounding hyperplanes to bound the attainable region there are several theoretical issues that revolve around the construction of bounds on attainability in the reactor synthesis problem. 
Throughout the presentation of results in this thesis, we have frequently recommended the need for a large number of hyperplanes to get good kinetic bounds. If we were given the opportunity to use such a large number to implement the method of bounding hyperplanes, we would come to a point where we have an "irreducible" [85] current polytope $\mathscr{C}$. We can refer to $\mathscr{C}$ as "irreducible" if no choice of newly introduced hyperplane serves to trim away compositions that are kinetically infeasible from the current polytope. Stated more formally, we say here that $\mathscr{C}$ is irreducible if, for any choice of hyperplane $H^{=}(\mathbf{n}, \gamma)$ such that the feed stream composition $\mathbf{c}^{\mathrm{f}}$ resides in $H^{2}(\mathbf{n}, \gamma)$ and such that $H^{<}(\mathbf{n}, \gamma) \bigcap \mathscr{C}$ is not empty, it is true that $\mathbf{n} \cdot \mathbf{r}\left(\mathbf{c}^{*}\right)<0$ for some $\mathbf{c}^{*} \in H^{\leq}(\mathbf{n}, \gamma) \bigcap \mathscr{C}[85]$. Such a set $\mathscr{C}$ can be bounded, in the limit, by an infinite number of bounding hyperplanes. When such a large number of hyperplanes are involved, the resulting polytope might not be a true polytope; it may have on its boundary a large number of smooth curved surfaces that are not planar. An example that suggests how this may happen is seen in Figure 7.6 where the 87-hyperplane bound approximates a smooth curve in the $2 \mathrm{D} \mathrm{VdV}$ problem.

For an irreducible bound $\mathscr{C}$, it is reasonable to conjecture that such a set not only bounds the attainable region but also coincides with it. The near-limiting polygonal bound in Figure 7.6 suggests how such a conjecture can be generally valid. However, there is no concrete proof that this is certainly the case. Even though some of the known mathematical features of the attainable region (such as those suggested by Feinberg [4-6]) are shared by an irreducible bounding set, there is no guarantee that the two will ultimately coincide. Since they do share common features, it may be possible to 
elaborate under what circumstances the bounding set and the attainable region are guaranteed to coincide, but this is a subject for future research.

One of the simpler extensions of the research presented in the thesis is to find kinetic bounds for non-isothermal systems. One of the effects of permitting nonisothermal conditions is that the rate constants for a chemical reaction generally vary with temperature. In this case, the species production rate function will be a function of composition as well as temperature.

For example, if we are studying the non-isothermal Van de Vusse reaction system, we have the following reaction network:

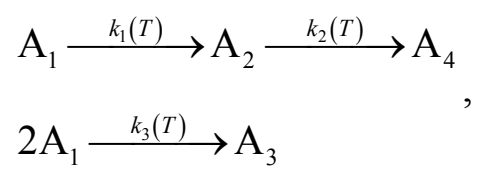

where the rate constants are functions of (local) temperature $T$. The species production rate function can be written as a function of composition and temperature as follows:

$$
\mathbf{r}(\mathbf{c}, T):=\left[-k_{1}(T) c_{1}-2 k_{3}(T) c_{1}^{2}, k_{1}(T) c_{1}-k_{2}(T) c_{2}, k_{3}(T) c_{1}^{2}, k_{2}(T) c_{2}\right] .
$$

If we assume a feed stream composition $\mathbf{c}^{\mathrm{f}}=[1,0,0,0]$ and if the temperatures within the system range from a minimum value of $T_{\min }$ to a maximum value of $T_{\max }$, then we can write the following constraints:

$$
\begin{aligned}
& c_{1} \geq 0 \\
& c_{2} \geq 0 \\
& c_{3} \geq 0 \\
& c_{1}+c_{2}+2 c_{3} \leq 1 \\
& T_{\min } \leq T \leq T_{\max }
\end{aligned}
$$


Similar to the isothermal problem, we begin with the stoichiometric constraints, compute extreme points, and introduce a new hyperplane at an extreme point. We can then try to move the hyperplane and check for two conditions: (1) the feed stream is on or on one side of the hyperplane and (2) on the other side of the hyperplane, within the temperature constraints given, the species production rate vectors at nonequilibrium compositions point to the side containing the feed. More specifically, $\mathbf{c}^{\mathrm{f}}$ lies on the hyperplane with normal $\mathbf{n}^{*}$ or on one side of it; on the other side of the hyperplane, within the current polytope, at every composition where

$$
\begin{aligned}
& \mathbf{r}(\mathbf{c}, T) \neq \mathbf{0}, \\
& T_{\min } \leq T \leq T_{\max }
\end{aligned}
$$

(i.e. nonequilibrium composition) the following condition is satisfied

$$
\begin{aligned}
& \mathbf{n} \cdot \mathbf{r}(\mathbf{c}, T)>\mathbf{0}, \\
& T_{\min } \leq T \leq T_{\text {max }}
\end{aligned}
$$

If so we can claim that the compositions on the other side of the hyperplane and within the current polytope are not kinetically feasible. The supporting argument is similar to that for the isothermal case.

It is reasonable to consider future directions for research that can deal with compressible systems. The pure reactor synthesis problem assumes incompressibility that comes into play in deriving the various mathematical features of the attainable region [4] and in deriving the method of bounding hyperplanes described in this thesis.

Incompressibility permits us to deal with convex regions in composition space. More precisely, when we have two incompressible mixture streams of compositions $\mathbf{c}_{1}$ and $\mathbf{c}_{2}$ then by mixing the two streams in varying proportions we can 
obtain any composition that is a convex combination of the two compositions. This type of mixing and the resultant convex composition sets obtained play an important role in the derivation of the method of bounding hyperplanes. Removing the incompressibility condition obviously make certain arguments in the derivation invalid. Thus it is not yet clear how comparable methods for the compressible case might evolve. This is a topic for future research. 


\section{APPENDIX A}

\section{COMPUTATIONAL TOOLS}

\section{A.1 CDDLIB}

The program cddlib is an efficient implementation [102] of the double description method [103] for generating all vertices (i.e. extreme points) of a convex polytope given by a system of linear inequalities:

$$
P:=\left\{\mathbf{x} \in \mathbb{R}^{\mathrm{d}}: \mathbf{b}-\mathbf{A} \cdot \mathbf{x} \geq \mathbf{0}\right\}
$$

where $\mathbf{A}$ is a matrix of real values with $m$ rows and $d$ columns; $\mathbf{b}$ is a matrix of real values $m$ rows and 1 column (i.e. we have $m$ inequalities.) The form of representation shown in (A.1) is also referred to as the H-representation. When reading from or writing to a computer file, the $i^{\text {th }}$ inequality is represented in a single line with a single space separating each value as follows:

$$
b_{i} \quad-A_{i}^{1} \quad-A_{i}^{2} \quad \ldots \ldots \ldots-A_{i}^{d}
$$

Another convention to represent a convex polytope is by using a set of

extreme points $\left\{v_{1}, v_{2}, \ldots . v_{n}\right\}$ where $v_{i} \in \mathbb{R}^{d}$. The convex polytope $P$ is represented as the convex hull of $n$ extreme points:

$$
P:=\operatorname{conv}\left(v_{1}, v_{2}, \ldots . . v_{n}\right)
$$


The representation of (A.3) is referred to as the $V$-representation and in a computer file the extreme points are written such that one extreme point is printed on each line with a single space separating each vector component.

According to the program author, Fukuda [91], the computation performed by cddlib is the transformation of an H-representation of a convex polytope to a V-representation. (It should be noted that the $\mathrm{H}$ and $\mathrm{V}$ representation described above is slightly modified from Fukuda's since we deal with closed convex polytopes while cddlib is written to handle additionally convex polyhedrons that are not necessarily closed.) The dual transformation problem of a $\mathrm{V}$-representation to a minimal $\mathrm{H}$-representation is often called the (convex) hull problem. "cddlib" comes with an LP code to solve the general linear programming (LP) problem to maximize (or minimize) a linear function over polyhedron $P$. It is this LP module that assists us in removing redundant halfspaces.

Suppose we are given a system of $m+1$ inequalities and asked to remove redundant inequalities from that system. We pick one inequality at a time and check if it is redundant with respect to the other $m$ inequalities. Assume [86] that we are given the following set of $m+1$ inequalities:

$$
\begin{aligned}
& \mathbf{A} \cdot \mathbf{x} \leq \mathbf{b} \\
& \mathbf{s}^{\prime} \cdot \mathbf{x} \leq t
\end{aligned}
$$

where $\mathbf{x} \in \mathbb{R}^{d}$ is the variable space, $\mathbf{A}$ is an $m \times d$ matrix, $\mathbf{b}$ is an $m \times 1$ matrix, $\mathbf{s}^{\prime}$ is a $1 \times d$ matrix and $t$ is any real value. To check if $\mathbf{s}^{\prime} \cdot \mathbf{x} \leq t$ is a redundant inequality we use the LP module of cddlib to perform the following optimization: 


$$
\begin{aligned}
f^{*}:= & \text { Maximize the objective function }: \mathbf{s}^{\prime} \cdot \mathbf{x} \\
& \text { Subject to the constraints }: \begin{array}{l}
\mathbf{A} \cdot \mathbf{x} \leq \mathbf{b} \\
\mathbf{s}^{\prime} \cdot \mathbf{x} \leq t+1
\end{array}
\end{aligned}
$$

The inequality $\mathbf{s}^{\prime} \cdot \mathbf{x} \leq t$ is redundant [86] if and only if the optimum value $f^{*}$ is less than or equal to $t$. Note that for a system of $m+1$ inequalities we need to perform at most $m+1$ linear optimizations to check and remove redundant inequalities.

\section{A.2 GNU Multiple Precision Arithmetic Library (GMP)}

GNU MP [92] is a portable library written in C for arbitrary precision arithmetic on integers, rational numbers, and floating-point numbers. It aims to provide the fastest possible arithmetic for all applications that need higher precision than is directly supported by the basic $\mathrm{C}$ types.

Many applications use just a few hundred bits of precision; but some applications may need thousands or even millions of bits. GMP is designed to give good performance for both, by choosing algorithms based on the sizes of the operands, and by carefully keeping the overhead at a minimum.

The speed of GMP is achieved by using fullwords (four bytes) as the basic arithmetic type, by using sophisticated algorithms, by including carefully optimized assembly code for the most common inner loops for many different CPUs, and by a general emphasis on speed (as opposed to simplicity or elegance). 
The rational arithmetic features in GMP were used with cddlib because numerical problems were observed when using the floating-point arithmetic. Fukuda suggests the use of GMP with rational arithmetic to overcome possible issues caused by numerical accuracy but this would be at the expense of computation time. All rational arithmetic functions assume operands have a canonical form, and canonicalize their result. The canonical form means that the denominator and the numerator have no common factors, and that the denominator is positive. Zero has the unique representation 0/1. In the Solver DLL implementation we use GMP solely inside the cdd module and hence all conversion to and from the rational number format is done inside that module alone.

\section{A.3 Solver Dynamic Link Libraries (Solver DLL)}

The Solver Dynamic Link Library (Solver DLL) [104] is a collection of software tools to solve linear, quadratic, nonlinear, nonsmooth optimization problems, and mixed-integer problems of varying size. The functions in the DLL can be accessed by programs written in Microsoft Visual C++, Visual Basic, Delphi Pascal and Fortran (these were supported in version 4.5 of Solver DLL but their status is not clear since version 6.0.)

Our interest, based on the algorithms developed for the method of bounding hyperplanes, is solely in the nonlinear optimization capabilities of Solver DLL. We have used two versions of the Solver DLL: the Small-Scale Solver DLL (default) and LGO Solver Engine 


\section{A.3.1 Small-Scale Solver DLL}

This version of the Solver DLL is the default version of this program available for purchase. The functions that perform nonlinear optimization have a vendorimposed limit of 250 constraints and 500 variables. The nonlinear optimization process is based on a generalized-reduced-gradient (GRG) method which is guaranteed to find a locally optimal solution which may or may not be the true global optimum within the specified constraints. To deal with this, an evolutionary solver [104] is incorporated into the Solver DLL. Though this still does not guarantee a global optimum, the vendors claim that this technique gives a value that has "high probability of being the global optimum."

Some of the important parameters that had to be modified frequently to obtain "highly probable" global optima are described below:

(1) Scaling: To deal with large differences in magnitude of the data relating to the constraints or objective function, the user could scale the optimization problem to minimize errors. We found that it was better to turn the scaling off since we would otherwise get erroneous results

(2) Differencing: The gradient-based method of Solver DLL requires the computing of partial derivatives of the objective function at certain values. To compute approximate first partial derivatives one could use either the forward differencing or central differencing methods. Forward differencing methods perturbed variables in one direction to compute the derivative while central differencing perturbed the variables in both the forward and backward directions from the 
current point to compute the derivative. Central differencing takes more computing time but we found it to give more accurate results.

(3) Sample size: This parameter determined the number of values for candidate solutions in the evolutionary solver. The larger the sample size the more likely one would find a truly global optimum. We set this value according to the size of our problem: a problem with two variables would do well with about 100 points, while a problem with three variables would need anywhere from 500 to 1000 points or more.

(4) Convergence: If the objective value in five consecutive iterations was found to change less than the tolerance value set by this parameter the GRG solver and the evolutionary solver assumes it has converged to a global optimum. For most problems we set this value to $10^{-5}$ but for more rigorous computations we set it to a lower value of $10^{-7}$.

(5) Initial value: At the onset of the optimization process, the solver uses an initial value from which it attempts to find a global optimum. This initial value is important because it might lead the solver to a locally optimal solution mistaking it for a globally optimum one. We found that the initial value worked well when we picked a value that simultaneously satisfies all the constraints involved in the optimization. Stated differently, a good choice for the initial point was one that lay inside the polytope formed by the current constraints. 


\section{A.3.2 LGO Global Solver Engine}

This program is based on the Lipschitz-continuous Global Optimization technique by Janos Pinter [105]. This solver had a vendor-imposed limit of 1000 constraints and 1000 variables. The LGO Global Solver uses a partial global search procedure, followed by a local search using local optimization techniques. These methods usually do guarantee that any solution found by the LGO Global Solver is at least locally optimal. Whereas the GRG method searches in a neighborhood of the initial value, the LGO Global Solver will search more systematically throughout the possible solution space. Some of the important parameters for this engine are as follows:

(1) Variable bounds: The upper and lower bounds on the variables were found to be the most important parameter in using this optimization technique. The LGO Global Solver searches for feasible and optimal solutions in the "box" defined by the upper and lower bounds on the variables while also taking into account the constraints. The tighter the bounds specified for the variables the more likely that the LGO Global Solver will find a feasible and potentially globally optimal solution. We normally set the variable limits to that limited by stoichiometry of the given reaction system.

(2) Global Search Method: The LGO Solver gives the option of using several global search strategies before it starts a local search for optima. Of the available methods we chose the Global Branch and Bound method to be most effective in giving us a truly global optimum. 
(3) Global Convergence: The search for global optima is controlled by this parameter. By default this value is set to $10^{-3}$ but we obtained better results when we set this to $10^{-4}$ and $10^{-5}$. Without going into the details, it is sufficient to understand that a lower value means that the LGO Solver spends relatively more time searching for a global optima before beginning the search for local optima. 


\section{BIBLIOGRAPHY}

1. Douglas, J. M., Conceptual Design of Chemical Processes. McGraw-Hill Book Company: 1988 .

2. Horn, F. J. M. In Attainable and non-attainable regions in chemical reaction technique, Chemical Reaction Engineering (Proceedings of the Third European Symposium), 1964; Pergamon Press, U.K.: 1964.

3. Van de Vusse, J. G., Plug-Flow Type Reactor versus Tank Reactor. Chemical Engineering Science 1964, 19, 994-997.

4. Feinberg, M.; Hildebrandt, D., Optimal reactor design from a geometric viewpoint-I. Universal properties of the attainable region. Chemical Engineering Science 1997, 52, (10), 1637-1665.

5. Feinberg, M., Optimal reactor design from a geometric viewpoint-III. Critical CFSTRs. Chemical Engineering Science 2000, 55, 3553-3565.

6. Feinberg, M., Optimal reactor design from a geometric viewpoint-II. Critical sidestream reactors. Chemical Engineering Science 2000, 55, 2455-2479.

7. Feinberg, M., Toward a Theory of Process Synthesis. Industrial and Engineering Chemistry Research 2002, 41, (16), 3751-3761.

8. Westerberg, A. W., A retrospective on design and process synthesis. Computers and Chemical Engineering 2004, 28, (4), 447-458.

9. Perkins, J. D. In Trends in process systems engineering, AIChE, Miami Beach (FL), 15-20 November, 1998; Miami Beach (FL), 1998.

10. Biegler, L. T.; Grossmann, I. E.; Westerberg, A. W., Systematic Methods of Chemical Process Design. Prentice Hall PTR: 1997.

11. Seider, W. D.; Seader, J. D.; Lewin, D. R., Product and Process Design Principles: Synthesis, Analysis and Evaluation. 2 ed.; John Wiley \& Sons, Inc.: 2004.

12. Douglas, J. M., A hierarchical decision procedure for process synthesis. AIChE Journal 1985, 31, (3), 353-362. 
13. Douglas, J. M.; Kirkwood, R. L., Design education in chemical engineering. Part 2: Using design tools. Chemical Engineering Education 1989, 23, (2), 120-127.

14. Douglas, J. M.; Malone, M. F.; Doherty, M. F., The interaction between separation systems synthesis and process synthesis. Computers and Chemical Engineering 1985, 9 , (5), 447-462.

15. Rossiter, A. P.; Douglas, J. M., Design and optimization of solids processes. Part 2: Optimization of crystallizer, centrifuge and dryer systems. Chemical Engineering Research and Design 1986, 64, (3), 184-190.

16. Rossiter, A. P.; Douglas, J. M., Design and optimization of solids processes. Part 1- A hierarchical design procedure for process synthesis of solids systems. Chemical Engineering Research and Design 1986, 64, (3), 175-183.

17. Grossman, I. E.; Santibanez, J., Applications of mixed-integer linear programming in process synthesis. Computers and Chemical Engineering 1980, 4, (4), 205-214.

18. Papoulias, S. A.; Grossmann, I. E., A structural optimization approach in process synthesis. II. Heat recovery networks. Computers and Chemical Engineering 1983, 7, (6), 707-721.

19. Papoulias, S. A.; Grossmann, I. E., A structural optimization approach in process synthesis. I. utility systems. Computers and Chemical Engineering 1983, 7, (6), 695-706.

20. Papoulias, S. A.; Grossmann, I. E., A structural optimization approach in process synthesis. III. Total processing systems. Computers and Chemical Engineering 1983, 7 , (6), 723-734.

21. Kocis, G. R.; Grossmann, I. E., Global optimization of nonconvex mixed-integer nonlinear programming (MINLP) problems in process synthesis. Industrial and Engineering Chemistry Research 1988, 27, (8), 1407-1421.

22. Raman, R.; Grossmann, I. E., Integration of logic and heuristic knowledge in MINLP optimization for process synthesis. Computers and Chemical Engineering 1992, 16, (3), 155-171.

23. Raman, R.; Grossmann, I. E., Symbolic integration of logic in mixed-integer programming techniques for process synthesis. Computers and Chemical Engineering 1993, 17, (9), 909-927.

24. Westerberg, A. W.; Stephanopoulos, G., Process Synthesis I. Branch and bound strategy with list techniques for the synthesis of separation schemes. Chemical Engineering Science 1975, 30, (8), 963-972. 
25. Westerberg, A. W.; Stephanopoulos, G., Studies in process synthesis I. Branch and bound strategy with list techniques for the synthesis of separation schemes. Chemical Engineering Science 1976, 31, (4), 325.

26. Stephanopoulos, G.; Westerberg, A. W., Studies in process synthesis II. Evolutionary synthesis of optimal process flowsheets. Chemical Engineering Science 1976, 31, (3), 195-204.

27. Linke, P.; Kokossis, A., On the robust application of stochastic optimization technology for the synthesis of reaction/separation systems. Computers and Chemical Engineering 2003, 27, (5), 733-758.

28. Yeomans, H.; Grossmann, I. E., A systematic modeling framework of superstructure optimization in process synthesis. Computers and Chemical Engineering 1999, 23, (6), 709-731.

29. Lee, S.; Grossman, I. E., New algorithms for nonlinear generalized disjunctive programming. Computers and Chemical Engineering 2000, 25, (7-8), 1153.

30. Lee, S.; Grossman, I. E., New algorithms for nonlinear generalized disjunctive programming. Computers and Chemical Engineering 2000, 24, (9-10), 2125-2141.

31. Vecchietti, A.; Lee, S.; Grossman, I. E., Modeling of discrete/continuous optimization problems: characterization and formulation of disjunctions and their relaxations. Computers and Chemical Engineering 2003, 27, (3), 433-448.

32. Adjiman, C. S.; Androulakis, I. P.; Floudas, C. A., Global optimization of MINLP problems in process synthesis and design. Computers and Chemical Engineering 1997, 21(Suppl., Joint 6th International Symposium on Process Systems Engineering and 30th European Symposium on Computer Aided Process Engineering, 1997), S445-S450.

33. Pintaric, Z. N.; Kravanja, Z., Two-level strategy for synthesis of processes under uncertainty condition. Zbornik Referatov s Posvetovanja Slovenski Kemijski Dnevi 1999, 452-459.

34. Pintaric, Z. N.; Kravanja, Z., Mixed-integer nonlinear programming problem process synthesis under uncertainty by reduced dimensional stochastic optimization. Industrial and Engineering Chemistry Research 1999, 38, (7), 2680-2698.

35. Pintaric, Z. N.; Kravanja, Z., The two-level strategy for MINLP synthesis of process flowsheets under uncertainty. Computers and Chemical Engineering 2000, 24, 195-201.

36. Dua, V.; Pistikopoulos, E. N., Multiparametric mixed integer optimization techniques for process synthesis under uncertainty. IChemE Research Event, A Two-Day Symposium 1998, 472-480. 
37. Dua, V.; Pistikopoulos, E. N., An outer-approximation algorithm for the solution of multiparametric MINLP problems. Computers and Chemical Engineering 1998, 22(Suppl., European Symposium on Computer Aided Process Engineering-8, 1998), S995-S958.

38. Dua, V.; Pistikopoulos, E. N., Optimization techniques for process synthesis and material design under uncertainty. Chemical Engineering Research and Design 1998, 76, (A3), 408-416.

39. Papalexandri, K. P.; Pistikopoulos, E. N., A decomposition-based approach for process optimization and simultaneous heat integration: application to an industrial process. Chemical Engineering Research and Design 1998, 76, (A3), 273-286.

40. Achenie, L. K. E.; Biegler, L. T., A superstructure based approach to chemical reactor synthesis. Computers and Chemical Engineering 1990, 14, (1), 23-40.

41. Drake, J. E.; Manousiouthakis, V., IDEAS approach to process network synthesis: minimum utility cost for complex distillation networks. Chemical Engineering Science 2002, 57, (15), 3095-3106.

42. Wilson, S.; Manousiouthakis, V., IDEAS approach to process network synthesis: application to multicomponent MEN. AIChE Journal 2000, 46, (12), 2408-2416.

43. Hostrup, M.; Gani, R.; Kravanja, Z.; Sorsak, A.; Grossmann, I., Integration of thermodynamic insights and MINLP optimization for the synthesis, design and analysis of process flowsheets. Computers and Chemical Engineering 2001, 25, (1), 73-83.

44. Friedler, F.; Varga, J. B.; Fan, L. T., Decision-mapping for design and synthesis of chemical processes: Application to reactor-network synthesis. AIChE Symposium Series 1995, 304(Fourth International Conference on Foundations of Computer-Aided Process Design, 1994, 246-250.

45. Friedler, F.; Varga, J. B.; Fan, L. T., Decision-mapping: a tool for consistent and complete decisions in process synthesis. Chemical Engineering Science 1995, 50, (11), $1755-1768$.

46. Friedler, F.; Tarjan, K.; Yuang, Y. W.; Fan, L. T., Graph-theoretic approach to process synthesis: Axioms and theorems. Chemical Engineering Science 1992, 47, (8), 1973-1988.

47. Friedler, F.; Tarjan, K.; Yuang, Y. W.; Fan, L. T., Graph-theoretic approach to process synthesis: polynomial algorithm for maximal structure generation. Computers and Chemical Engineering 1993, 17, (9), 929-942.

48. Bek-Pedersen, E.; Gani, R.; Levaux, O., Determination of optimal energy efficient separation schemes based on driving forces. Computers and Chemical Engineering 2000, $24,(2-7), 253-259$. 
49. Bek-Pedersen, E.; Hostrup, M.; Gani, R., Using driving force based separation efficiency curves within an integrated system for process synthesis/design. ComputerAided Chemical Engineering 2000, 8(European Symposium on Computer Aided Process Engineering-10, 2000), 955-960.

50. Jaksland, C.; Gani, R., An integrated approach to process/product design and synthesis based on properties-process relationship. Computers and Chemical Engineering 1996, 20(Suppl. A, European Symposium on Computer Aided Process Engineering-6, 1996), S151-S156.

51. Schembecker, G.; Simmrock, K. H., Heuristic-numeric process synthesis with Prosyn. AIChE Symposium Series 1996, 312(Intelligent Systems in Process Engineering), 275278.

52. Kravanja, Z.; Grossman, I. E., PROSYN:- an automated topology and parameter process synthesizer. Computers and Chemical Engineering 1993, 17(Suppl., European Symposium on Computer Aided Process Engineering-2, 1992), S87-S94.

53. Schembecker, G.; Droege, T.; Westhaus, U.; Simmrock, K. H., READPERTdevelopment, selection and design of chemical reactors. Chemical Engineering and Processing 1995, 34, (3), 317-322.

54. Schembecker, G.; Droege, T.; Westhaus, U.; Simmrock, K. H., A heuristic-numeric consulting system for the choice of chemical reactors. AIChE Symposium Series 1995, 304(Fourth International Conference on Foundations of Computer-Aided Process Design), 336-339.

55. O'Grady, A. R. F.; Bogle, I. D. L.; Fraga, E. S., Interval analysis in automated design for bounded solutions. Chemical Papers 2001, 55, (6), 376-381.

56. Fraga, E. S.; Hagemann, J.; Estrada-Villagrana, A.; Bogle, I. D. L., Incorporation of dynamic behavior in an automated process synthesis system. Computers and Chemical Engineering 2000, 24, (2-7), 189-194.

57. Fraga, E. S.; Wang, K.; Salhi, A., Interactivity and automated process design. Chemical Engineering and Technology 2003, 26, (8), 823-827.

58. Fraga, E. S.; Steffens, M. A.; Bogle, I. D. L.; Hind, A. K., An object-oriented framework for process synthesis and optimization. AIChE Symposium Series 2000, 323(Foundations of Computer-Aided Process Design), 446-449.

59. Chitra, S. P.; Govind, R., Synthesis of optimal serial reactor structures for homogeneous reactions. 1. Isothermal reactors. AIChE Journal 1985, 31, (2), 177-184.

60. Chitra, S. P.; Govind, R., Synthesis of Optimal serial reactor structures for homogeneous reactions. 2. Non-isothermal reactors. AIChE Journal 1985, 31, (2), 185194. 
61. Nisoli, A.; Malone, M. F.; Doherty, M. F., Attainable regions for reaction with separation. AIChE Journal 1997, 43, (2), 374-387.

62. Nicol, W.; Hildebrandt, D.; Glasser, D., Process synthesis for reaction systems with cooling via finding the attainable region. Computers and Chemical Engineering 1997, 21(Suppl., Joint 6th International Symposium on Process Systems Engineering and 30th European Symposium on Computer Aided Process Engineering), S35-S40.

63. Rooney, W. C.; Biegler, L. T., Multiperiod reactor network synthesis. Computers and Chemical Engineering 2000, 24, (9-10), 2055-2068.

64. Lakshmanan, A.; Biegler, L. T., Synthesis of optimal chemical reactor networks with simultaneous mass integration. Industrial and Engineering Chemistry Research 1996, 35, (12), 4523-4536.

65. Lakshmanan, A.; Biegler, L. T., Synthesis of optimal chemical reactor networks. Industrial and Engineering Chemistry Research 1996, 35, (4), 1344-1353.

66. Pahor, B.; Kravanja, Z.; Bedenik, N. I., Synthesis of reactor networks in overall process flowsheets within the multilevel MINLP approach. Computers and Chemical Engineering 2001, 25, (4-6), 765-774.

67. Pahor, B.; Kravanja, Z., Simultaneous solution and MINLP synthesis of DAE process problems: PFR networks in overall processes. Computers and Chemical Engineering 1995, 19(Suppl., European Symposium on Computer Aided Process Engineering--5), S181-S188.

68. Pahor, B.; Irsic, N.; Kravanja, Z., MINLP synthesis and modified attainable region analysis of reactor networks in overall process schemes using more compact reactor superstructure. Computers and Chemical Engineering 2000, 24, (2-7), 1403-1408.

69. Schweiger, C. A.; Floudas, C. A., Optimization framework for the synthesis of chemical reactor networks. Industrial and Engineering Chemistry Research 1999, 38, (3), 744-766.

70. Linke, P.; Kokossis, A., Advanced process systems design technology for pollution prevention and waste treatment. Advances in Environmental Research 2004, 8, (2), 229245.

71. Uppaluri, R.; Smith, R.; Linke, P.; Kokossis, A., Optimal design of gas permeation membrane and membrane adsorption hybrid systems. Computer-Aided Chemical Engineering 2002, 10(European Symposium on Computer Aided Process Engineering12, 2002), 367-372.

72. Nisoli, A.; Doherty, M. F.; Malone, M. F., Feasible regions for step-growth melt polycondensation systems. Industrial and Engineering Chemistry Research 2004, 43, (2), 428-440. 
73. Smith, R. L.; Malone, M. F., Attainable regions for polymerization reaction systems. Industrial and Engineering Chemistry Research 1997, 36, (4), 1076-1084.

74. Smith, R. L.; Malone, M. F., Feasible compositions for random copolymerizations. Polymer Engineering and Science 2001, 41, (2), 145-154.

75. Milne, D.; Glasser, D.; Hildebrandt, D.; Hausberger, B., Application of the attainable region concept to the oxidative dehydrogenation of 1-Butene in inert porous membrane reactors. Industrial and Engineering Chemistry Research 2004, 43, (8), 1827-1831.

76. Kauchali, S.; Hausberger, B.; Hildebrandt, D.; Glasser, D.; Biegler, L. T., Automating reactor network synthesis: finding a candidate attainable region for the water-gas shift (WGS) reaction. Computers and Chemical Engineering 2004, 28, (1-2), 149-160.

77. Lakshmanan, A.; Rooney, W. C.; Biegler, L. T., A case study for reactor network synthesis: the vinyl chloride process. Computers and Chemical Engineering 1999, 23, (45), 479-495.

78. Lakshmanan, A.; Biegler, L. T., A case study for reactor network synthesis: the vinyl chloride process. Computers and Chemical Engineering 1997, 21(Joint 6th International Symposium on Process Systems Engineering and 30th European Symposium on Computer Aided Process Engineering), S785-S790.

79. Steffens, M. A.; Fraga, E. S.; Bogle, I. D. L., Multicriteria process synthesis for generating sustainable and economic bioprocesses. Computers and Chemical Engineering 1999, 23, (10), 1455-1467.

80. Steffens, M. A.; Fraga, E. S.; Bogle, I. D. L., Synthesis of bioprocesses using physical properties data. Biotechnology and Bioengineering 2000, 68, (2), 218-230.

81. Feinberg, M.; Ellison, P., General kinetic bounds on productivity and selectivity in reactor-separator systems of arbitrary design: Principles. Industrial and Engineering Chemistry Research 2001, 40, (14), 3181-3194.

82. Feinberg, M., Chemical Reaction Network Structure and the stability of complex isothermal reactors- I. The deficiency zero and deficiency one theorems. Chemical Engineering Science 1987, 42, (10), 2229-2268.

83. Feinberg, M. Lectures on Chemical Reaction Networks. http://www.che.eng.ohiostate.edu/ feinberg/LecturesOnReactionNetworks/

84. Shinnar, R.; Feng, C. A., Structure of Complex Catalytic Reactions: Thermodynamic Constraints in Kinetic Modeling and Catalyst Evaluation. Industrial \& Engineering Chemistry Fundamentals 1985, 24, (2), 153-170. 
85. Abraham, T. K.; Feinberg, M., Kinetic Bounds on Attainability in the Reactor Synthesis Problem. Industrial and Engineering Chemistry Research 2004, 43, (2), 449457.

86. Fukuda, K. Frequently Asked Questions in Polyhedral Computation.

http://www.ifor.math.ethz.ch/ fukuda/polyfaq/polyfaq.html

87. Painik, M. J., Fundamentals of convex analysis: duality, separation, representation and resolution. 1993.

88. Eggleston, H. G., Convexity. Cambridge University Press: 1966.

89. Fukuda, K. cdd/cdd+ Reference Manual.

http://www.cs.mcgill.ca/ fukuda/soft/cddman/cddman.html

90. Lang, S., Introduction to Linear Algebra. 2 ed.; Springer-Verlag New York Inc.: 1988.

91. Fukuda, K. cddlib package, 093d; 2005.

92. Granlund, T. GNU Multiple Precision Arithmetic Library. http://www.swox.com/gmp/

93. Conti, G. A. P.; Paterson, W. R. In Chemical reactors in process synthesis, Process Systems Engineering, PSE '85, 1985; 1985.

94. Balakrishna, S.; Biegler, L. T., Chemical reactor network targeting and integration: an optimization approach. Advances in Chemical Engineering 1996, 23, 247-300.

95. Floudas, C. A.; Grossmann, I. E., Algorithmic approaches to process synthesis: Logic and global optimization. AIChE Symposium Series 1995, 304(Fourth International Conference on Foundations of Computer-Aided Process Design, 1994), 198-221.

96. Glasser, D.; Hildebrandt, D., Reactor and process synthesis. Computers and Chemical Engineering 1997, 21(Suppl., Joint 6th International symposium on Process Systems engineering and 30th European Symposium on Computer Aided Process Engineering), S775-S783.

97. Waghmare, R. S.; Moharir, A. S., Optimal non-isothermal reactor network for Van de Vusse reaction. International Journal of Chemical Reactor Engineering 2005, 3.

98. Brooks, B. W., Product yields from the Van de Vusse reaction scheme : use of semibatch reactors. Chemical Engineering Science 1990, 45, (6).

99. Feinberg, M., Recent results in optimal reactor synthesis via attainable region theory. Chemical Engineering Science 1999, 54, 2535-2543. 
100. Tang, Y. Doctoral Thesis. The Ohio State University, Columbus, 2005.

101. Turton, R.; Baile, R. C.; Whiting, W. B.; Shaeiwitz, J. A., Analysis, Synthesis, and Design of Chemical Processes. Prentice Hall, PTR: 1998.

102. Fukuda, K.; Prodon, A., Double description method revisited. In Combinatorics and Computer Science, Deza, M.; Euler, R.; Manoussakis, Y., Eds. Springer: 1996; Vol. 1120.

103. Motzkin, T. S.; Raiffa, H.; Thompson, G.; Thrall, R. M., The double description method. In Contributions to theory of games, Kuhn, H. W.; Tucker, A. W., Eds. Princeton University Press: Princeton, 1953; Vol. 2.

104. Systems, F., Solver User's Guide: Dynamic Link Library, Version 3.5. In 2003.

105. Pinter, J. D., Global Optimization in Action : Continuous and Lipschitz Optimization: Algorithms, Implementations and Applications (Nonconvex Optimization and Its Applications). Springer: 1995. 